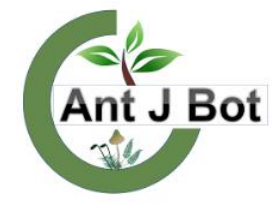

Received : 02.03.2020

Accepted : 18.03 .2020

Online : 20.03 .2020

\section{The explorations on the flora of Tunceli (Turkey)}

\author{
Metin ARMAĞAN \\ ${ }^{1}$ Aydın Adnan Menderes University, Buharkent Vocational School, Aydin, Turkey \\ *metinarmagan@gmail.com
}

\section{Tunceli (Türkiye) florası üzerine araştırmalar}

\begin{abstract}
The study was carried out until 2018 intermittently, on condition that majority in 2014. The study area is located between Erzincan, Malatya, Elazığ and Bingöl cities in the Eastern Anatolia. Its whole is placed in the Irano-Turanian phytogeographic region and, in B7 and B8 squares according to Davis' Grid System. During the study, 2950 samples were collected and 1559 of them were identified. The identification of these samples resulted 74 families, 386 genera and 922 taxa. The distribution of taxa by phytogeographic regions is as follows; Irano-Turanian elements 397 (43.1 \%), Mediterranean elements $20(2.2 \%)$, Europe-Siberian elements 40 (4.3\%), East Mediterranean elements 14 (1.5\%), Euxine element $13(1.4 \%)$, Hyrcano-Euxine elements $3(0.3 \%)$, Sarmatian $1(0.1 \%)$, the cosmopolite or unknown distribution $434(47.1 \%)$. The number of endemic taxa is 150 (endemism ratio $16.3 \%$ ).
\end{abstract}

Key words: Biodiversity, Eastern Anatolia, endemic, phytogeographic region, taxon

Özet: Çalışma, çoğunluğu 2014 yılında olması kaydıyla aralıklı olarak 2018 yılına kadar gerçekleştirilmiştir. Çalışma alanı, Doğu Anadolu'daki Erzincan, Malatya, Elazı̆̆ ve Bingöl şehirleri arasında yer almaktadır. Çalışma alanın tamamı İran-Turan fitocoğrafik bölgesinde ve Davis'in Kareleme Sistemi'ne göre B7 ve B8 karelerinde bulunur. Çalışma boyunca toplanan 2950 örneğin 1559 tanesi teşhis edilmiştir. Bu örneklerin teşhis edilmesiyle, 74 familyaya ait 386 cins ve 922 takson bulunmuştur. Taksonların fitocoğrafik bölgelere göre dağılımı şöyledir; 397 (\% 43.1)'si İran-Turan elementi, 20 (\% 2.2)'si Akdeniz elementi, 40 (\% 4.3)'1 Avrupa-Sibirya elementi, 14 (\% 1.5)'ü Doğu Akdeniz elementi, 13 (1.4)'ü Karadeniz elementi, 3 (\% 0.3)'ü Hirkanya-Karadeniz elementi, 1 (\% 0.1)'i Sarmasya elementi, 434 (\% 47.1)'ü kozmopolit veya fitocoğrafik bölgesi bilinmeyen. 150 takson endemiktir (endemizm oran1 \% 16.3).

Anahtar Kelimeler: Biyoçeşitlilik, Doğu Anadolu, endemik, fitocoğrafik bölge, takson

Citation: Armağan M (2020). The explorations on flora of Tunceli (Turkey). Anatolian Journal of Botany 4(1): 11-56.

\section{Introduction}

The flora of Turkey has high endemism ratio and rich plant diversity due to its location at the connections of Europe, the Middle East, Central Asia, and Africa, and also the interaction among Mediterranean, Irano-Turanian and Europe-Siberian phytogeographic regions (Davis, 1971; Gür, 2016).

Tunceli is located on Anatolian diagonal in Eastern region of Turkey (Davis, 1971). Geography of Tunceli composes of mountains ranging from $1500 \mathrm{~m}$ to $3000 \mathrm{~m}$, deep valleys, plateaus and very small amount of plains. There are a lot of small and large streams flowing between mountains. This area is surrounded by Keban Dam, Pülümür River and the branches of Euphrates River. The geographic texture and sparse human population provide the protection of natural life. Tunceli has the least agriculture area (114.000 ha) in Eastern Anatolia. So, sheep breeding is very important in this area. The grasslands are used for winter fodder and grazing in summer (Öztürk et al., 2015).

In Tunceli, the average annual temperature is $12.8^{\circ} \mathrm{C}$ and the precipitation is about $857.9 \mathrm{~mm}$ per year. July and August are the driest months, and the average monthly precipitation falls under $4 \mathrm{~mm}$ during this period. The greatest amount of precipitation occurs in December, with an average of $130.8 \mathrm{~mm}$. July is the warmest month with an average of $27.2^{\circ} \mathrm{C}$. The lowest average temperatures in the year occur in January, when it is around $-1.9^{\circ} \mathrm{C}$. The climate of the region is classified as Csa (dry and hot summer Mediterranean) by the Köppen-Geiger Climate Classification system (MGM, 2010).

Munzur Valley National Park, which is one of the Turkey's largest national parks, was declared as a national park in 1971, and serves as homel for many plant and animal species.

The study aims to determine the plant diversity of Tunceli province and to contribute to the Flora of Turkey.

\section{Materials and Method}

During fieldworks of this research, about 2950 plant samples were collected within Tunceli province during the vegetation seasons until 2018 intermittently, on condition that majority in 2014. Moreover, photos were taken in their natural habitats, GPS coordinates were recorded with their localities and habitat information. The collected samples were dried based on herbarium methods. Flora of Turkey and the East Aegean Islands (Davis, 1965-1985; Davis et al., 1988) was used as the initial reference for identification. The plant names and authors were checked from "Bizim Bitkiler (2013)", "Plants of the World Online (POWO, 2019)", "World Flora Online (WFO, 2020)", and "International Plant Names Index (IPNI, 2020)". Prepared samples with respect to herbarium techniques were stored in the AYDN, VANF, KNYA, and NGBB herbaria (Thiers, 2016).

\section{Results}

Nine hundred and twenty two plant taxa belonging to 386 genera and 74 families according to Stevens (2001) were 
determined and listed in alphabetical order in Table 1. Previously presented taxa (Armağan, 2018) were not included in the list.

Davis' Grid System was used in localities (Davis, 1965). The plant names, collector numbers, localities, the phytogeographic regions, and the endemism status etc. of the taxa were also given in the table. (Stevens, 2001).

The abbreviations used in the Table 1 as follow;

The directions $(\mathrm{E}=$ East, $\mathrm{W}=$ West, $\mathrm{N}=$ North, $\mathrm{S}=$ South)

$\mathrm{m}=$ meter

$\mathrm{km}=$ kilometer

nh. $=$ neighbourhood

v. = village

Mt. = mountain

$*=$ endemic
Ir.-Tur. = Irano - Turanian phytogeographical region Medit. = Mediterranean phytogeographical region Hyr.-Eux. = Hyrcano-Euxine phytogeographical region Eux. $=$ Euxine phytogeographical region

Euro.-Sib. $=$ Euro - Siberian phytogeographical region E.Medit. = East Mediterranean phytogeographical region

Sarmat. $=$ Sarmatian phytogeographical region

Cosmp. $=$ Cosmopolite

Unk. = Unknown

Alt. $=$ Altidue

Obs. $=$ Observation

End. $=$ Endemic

Col.No $=$ Collector number

Rcd. $=$ New record for Tunceli province

Table 1. Plant list of the area.

\begin{tabular}{|c|c|c|c|c|c|c|c|}
\hline Scientific name & Col.No & Localite & Alt. & Date & Habit & Note & Region \\
\hline \multicolumn{8}{|l|}{ ACANTHACEAE Juss. } \\
\hline Acanthus dioscoridis L. & 4434 & $\begin{array}{l}\text { B7 Tunceli: Center, } 3.5 \mathrm{~km} \text { from Aktuluk to } \\
\text { Buğulu }\end{array}$ & 1065 & 06.06 .2014 & \begin{tabular}{|l|} 
Degraded \\
oaks forest
\end{tabular} & & Unk. \\
\hline \multicolumn{8}{|l|}{ AMARANTHACEAE Juss. } \\
\hline \multirow{3}{*}{ Amaranthus albus L. } & 5735 & $\begin{array}{l}\text { B7 Tunceli: Mazgirt, } 2 \mathrm{~km} \text { from Akdüven to } \\
\text { Güleç }\end{array}$ & 1100 & 05.08 .2014 & \multirow{3}{*}{$\begin{array}{l}\text { Steppe, } \\
\text { ruderal }\end{array}$} & & \multirow{3}{*}{ Unk. } \\
\hline & 6127 & B7 Tunceli: Mazgirt, S of Dedebağ v. & 860 & 26.08 .2014 & & & \\
\hline & 5954 & B7 Tunceli: Mazgirt, Eltihatun & 1414 & 09.08 .2014 & & & \\
\hline Amaranthus retroflexus $\mathrm{L}$. & 5767 & $\begin{array}{l}\text { B7 Tunceli: Pülümür, } 45 \mathrm{~km} \text { from Tunceli to } \\
\text { Pülümür (Pülümür valley) }\end{array}$ & 1200 & 06.08 .2014 & Steppe & & Unk. \\
\hline $\begin{array}{l}\text { Beta corolliflora Zosimovic ex } \\
\text { Buttler }\end{array}$ & 6173 & $\begin{array}{l}\text { B7 Tunceli: } 10 \mathrm{~km} \text { from Pülümür - Erzincan } \\
\text { mainroad to Süleymanuşağ1 village }\end{array}$ & 2210 & 27.08 .2014 & Steppe & Red. & Ir.-Tur. \\
\hline \multirow{3}{*}{ Beta macrorhiza Steven } & 5941 & $\begin{array}{l}\text { B7 Tunceli: Ovacık, } 5 \mathrm{~km} \text { from Işıkvuran to } \\
\text { Ağaçpınar }\end{array}$ & 2133 & 08.08 .2014 & \multirow{3}{*}{$\begin{array}{l}\text { Steppe, } \\
\text { near } \\
\text { streamside }\end{array}$} & \multirow{3}{*}{ Rcd. } & \multirow{3}{*}{ Ir.-Tur. } \\
\hline & 4287 & B7 Tunceli: Ovacık, Işıkvuran v. & 1710 & 04.06 .2014 & & & \\
\hline & 4421 & $\begin{array}{l}\text { B7 Tunceli: Pülümür, between Kabadal and } \\
\text { Sağlamtaş }\end{array}$ & 1540 & 05.06 .2014 & & & \\
\hline Chenopodium album $\mathrm{L}$. & 6126 & B7 Tunceli: Mazgirt, S of Dedebağ v. & 860 & 26.08 .2014 & Steppe & & Unk. \\
\hline \multirow{2}{*}{ Chenopodium botrys L. } & 4486 & $\begin{array}{l}\text { B7 Tunceli: Çemişgezek, } 2 \mathrm{~km} \text { from } \\
\text { Bulgurtepe to Payamdüzü }\end{array}$ & 1060 & 06.06 .2014 & \multirow{2}{*}{$\begin{array}{l}\text { Ruderal, } \\
\text { steppe }\end{array}$} & & \multirow{2}{*}{ Unk. } \\
\hline & 4696 & $\begin{array}{l}\text { B7 Tunceli: Hozat, } 4.5 \mathrm{~km} \text { from Uzundal to } \\
\text { Yüceldi }\end{array}$ & 1970 & 17.06 .2014 & & & \\
\hline Chenopodium foliosum Asch. & Obs. & $\begin{array}{l}\text { B7 Tunceli: Hozat, } 15 \mathrm{~km} \text { from Ovacık to } \\
\text { Hozat (NE of Halitpınar v.) }\end{array}$ & 1800 & 17.06.2014 & Steppe & & Unk. \\
\hline \multirow{2}{*}{$\begin{array}{l}\text { Chenopodium opulifolium } \\
\text { Schrad. ex W.D.J.Koch \& Ziz }\end{array}$} & 4584 & B7 Tunceli: Mazgirt, $1 \mathrm{~km} \mathrm{E}$ of Geçitveren v. & 1270 & 16.06 .2014 & \multirow{2}{*}{$\begin{array}{l}\text { Ruderal, } \\
\text { steppe }\end{array}$} & \multirow[b]{2}{*}{ Rcd. } & \multirow[b]{2}{*}{ Unk. } \\
\hline & $\mathbf{5 7 5 2}$ & $\begin{array}{l}\text { B7 Tunceli: Nazımiye, Pülümür Valley, } 38 \\
\text { km from Tunceli to Pülümür }\end{array}$ & 1150 & 06.08 .2014 & & & \\
\hline $\begin{array}{l}\text { Chenopodium sosnowskyi } \\
\text { Kapeller }\end{array}$ & 5557 & B8 Tunceli: Pülümür, $5 \mathrm{~km} \mathrm{~S}$ of Derindere & 2030 & 22.07 .2014 & Steppe & Red. & Unk. \\
\hline \multirow[t]{2}{*}{ Chenopodium urbicum $\mathrm{L}$. } & 6274 & $\begin{array}{l}\text { B7 Tunceli: Mazgirt, between Beylermezras1 } \\
\text { and Göktepe (side of Munzur stream) }\end{array}$ & 842 & 19.09.2014 & \multirow{2}{*}{$\begin{array}{l}\text { Dried river } \\
\text { bad, steppe }\end{array}$} & \multirow[t]{2}{*}{ Rcd. } & \multirow[t]{2}{*}{ Unk. } \\
\hline & 6051 & B7 Tunceli: Mazgirt, around of Kale v. & 1590 & 15.08 .2014 & & & \\
\hline Chenopodium vulvaria $\mathrm{L}$. & 5894 & $\begin{array}{l}\text { B7 Tunceli: Pülümür, } 42 \mathrm{~km} \text { from Tunceli to } \\
\text { Pülümür (Pülümür Valley), across of } \\
\text { Ağlayan Kayalar }\end{array}$ & 1570 & 07.08 .2014 & Steppe & Rcd. & Unk. \\
\hline \multirow[b]{2}{*}{$\begin{array}{l}\text { Noaea mucronata (Forssk.) Asch. } \\
\& \text { Schweinf. }\end{array}$} & 5349 & B7 Tunceli: Nazımiye, E of Ramazanköy v. & 1440 & 20.07 .2014 & \multirow{2}{*}{$\begin{array}{l}\text { Eroded } \\
\text { slopes, } \\
\text { steppe }\end{array}$} & & \multirow[b]{2}{*}{ Unk. } \\
\hline & 6341 & $\begin{array}{l}\text { B7 Tunceli: Ovacık, } 8 \mathrm{~km} \text { from Işıkvuran to } \\
\text { Ağaçpınar }\end{array}$ & 2150 & 21.09.2014 & & & \\
\hline \multirow{2}{*}{$\begin{array}{l}\text { Noaea tournefortii (Jaub.\& } \\
\text { Spach) Moq. }\end{array}$} & 5000 & $\begin{array}{l}\text { B7 Tunceli: Ovacık, Ziyaret v., } \mathrm{N} \text { of Munzur } \\
\text { Gözeleri }\end{array}$ & 1400 & 20.06 .2014 & \multirow{2}{*}{$\begin{array}{l}\text { Rocky } \\
\text { areas, } \\
\text { steppe } \\
\end{array}$} & & \multirow[t]{2}{*}{ Unk. } \\
\hline & 5570 & B8 Tunceli: Pülümür, 2 km N of Elmalı & 1400 & 22.07 .2014 & & & \\
\hline \multirow[t]{2}{*}{ Salsola tragus L. } & 5114 & $\begin{array}{l}\text { B7 Tunceli: Pertek, } 3 \mathrm{~km} \text { from Elazı ğ-Pertek- } \\
\text { Çemişgezek crossroads to Çemişgezek ( } 1 \mathrm{~km} \\
\text { S of Çorovan v.) }\end{array}$ & 870 & 28.06.2014 & \multirow[t]{2}{*}{ Steppe } & \multirow[t]{2}{*}{ Rcd. } & \multirow[t]{2}{*}{ Unk. } \\
\hline & 5963 & $\begin{array}{l}\text { B7 Tunceli: Pertek, } 9 \mathrm{~km} \text { from Pertek to } \\
\text { Çemişgezek, } 2.5 \mathrm{~km} \text { before Singeç bridge }\end{array}$ & 875 & 13.08 .2014 & & & \\
\hline
\end{tabular}




\begin{tabular}{|c|c|c|c|c|c|c|c|}
\hline Cotinus coggygria Scop. & 4202 & $\begin{array}{l}\text { B7 Tunceli: Ovacık, } 1.5 \mathrm{~km} \text { from Ovacık- } \\
\text { Tunceli-Yakatarla crossroads to Yakatarla v. }\end{array}$ & 1320 & 04.06 .2014 & $\begin{array}{l}\text { Oak forest, } \\
\text { riverside }\end{array}$ & & Unk. \\
\hline Pistacia palaestina Boiss. & 4984 & B7 Tunceli: Ovacık, W of Ziyaret v. & 1340 & 20.06 .2014 & Steppe & & E.Medit. \\
\hline Rhus coriaria $\mathrm{L}$. & 6240 & $\begin{array}{l}\text { B7 Tunceli: Nazımiye, Pülümür Valley, } 24 \\
\text { km from Tunceli to Pülümür }\end{array}$ & 1017 & 05.09 .2014 & $\begin{array}{l}\text { Riverside, } \\
\text { oak forest }\end{array}$ & & Unk. \\
\hline \multicolumn{8}{|l|}{ APIACEAE Lindl. } \\
\hline Actinolema macrolema Boiss. & 3725 & B7 Tunceli: Center, $1 \mathrm{~km} \mathrm{~S}$ of Aktuluk & 930 & 22.05 .2014 & Steppe & & Ir.-Tur. \\
\hline $\begin{array}{l}\text { Anthriscus nemorosa (M.Bieb.) } \\
\text { Spreng. }\end{array}$ & 6528 & $\begin{array}{l}\text { B7 Tunceli: Nazımiye, } 12 \mathrm{~km} \mathrm{NE} \mathrm{of} \\
\text { Büyükyurt (Hakis) v., (5 km SE of } \\
\text { Aşağımarkosör) }\end{array}$ & 1926 & 01.06 .2015 & Riverside & & Unk. \\
\hline \multirow{4}{*}{ Artedia squamata $\mathrm{L}$. } & 4087 & $\begin{array}{l}\text { B7 Tunceli: Mazgirt, between Tunceli and } \\
\text { Kovancilar, around of Kuşcu }\end{array}$ & 867 & 02.06 .2014 & \multirow{4}{*}{$\begin{array}{l}\text { Steppe, } \\
\text { ruderal, in } \\
\text { gardens, } \\
\text { field side }\end{array}$} & & \multirow{4}{*}{ Unk. } \\
\hline & 4531 & B7 Tunceli: Mazgirt, 1 km W of Kayacı v. & 990 & 16.06 .2014 & & & \\
\hline & 5120 & $\begin{array}{l}\text { B7 Tunceli: Pertek, } 3 \mathrm{~km} \text { from Elazığ-Pertek- } \\
\text { Çemişgezek crossroads to Çemişgezek ( } 1 \mathrm{~km} \\
\text { S of Çorovan v.) }\end{array}$ & 870 & 28.06 .2014 & & & \\
\hline & 5262 & B7 Tunceli: Pertek, Camikebir nh. & 1100 & 19.07 .2014 & & & \\
\hline \multirow{2}{*}{ Astrodaucus orientalis (L.) Drude } & 5271 & $\begin{array}{l}\text { B7 Tunceli: Pertek, } 3 \mathrm{~km} \text { from Pertek to } \\
\text { Tunceli }\end{array}$ & 1257 & 19.07 .2014 & \multirow{2}{*}{ Steppe } & \multirow{2}{*}{ Rcd. } & \multirow{2}{*}{ Ir.-Tur. } \\
\hline & 5573 & $\begin{array}{l}\text { B8 Tunceli: Pülümür, between Kuzulca and } \\
\text { Elmalı }\end{array}$ & 1360 & 22.07.2014 & & & \\
\hline \multirow{3}{*}{ Bunium elegans (Fenzl) Freyn } & 4741 & $\begin{array}{l}\text { B7 Tunceli: Ovacık, } 11 \mathrm{~km} \text { from Ovacık to } \\
\text { Hozat, } 1 \mathrm{~km} \text { before junction of Bilgeç } \mathrm{v} \text {. }\end{array}$ & 1820 & 17.06 .2014 & \multirow{3}{*}{ Steppe } & \multirow{3}{*}{ Rcd. } & \multirow{3}{*}{ Unk. } \\
\hline & 4988 & B7 Tunceli: Ovacık, W of Ziyaret v. & 1340 & 20.06 .2014 & & & \\
\hline & 3873 & $\begin{array}{l}\text { B7 Tunceli: Pertek, } 5 \mathrm{~km} \text { from Pertek to } \\
\text { Çemişgezek }\end{array}$ & 866 & 24.05 .2014 & & & \\
\hline \multirow{2}{*}{$\begin{array}{l}\text { Bunium microcarpum (Boiss.) } \\
\text { Freyn \& Bornm. ex Freyn subsp. } \\
\text { buorgaei (Boiss.) Hedge \& } \\
\text { Lamond }\end{array}$} & 4205 & $\begin{array}{l}\text { B7 Tunceli: Ovacık, } 1.5 \mathrm{~km} \text { from Ovacık- } \\
\text { Tunceli-Yakatarla crossroads to Yakatarla v. }\end{array}$ & 1320 & 04.06 .2014 & \multirow[b]{2}{*}{ Steppe } & & \multirow[b]{2}{*}{ Ir.-Tur. } \\
\hline & 4065 & $\begin{array}{l}\text { B7 Tunceli: Ovacık, } 51 \mathrm{~km} \text { from Tunceli to } \\
\text { Ovacık (Munzur Valley), } 7 \mathrm{~km} \text { to Ovacık }\end{array}$ & 1210 & 26.05 .2014 & & & \\
\hline $\begin{array}{l}\text { Bunium microcarpum (Boiss.) } \\
\text { Freyn \& Bornm. ex Freyn subsp. } \\
\text { microcarpum }\end{array}$ & 4927 & $\begin{array}{l}\text { B7 Tunceli: Ovacık, } 8 \mathrm{~km} \text { from Eskigedik to } \\
\text { Işıkvuran }\end{array}$ & 2120 & 19.06.2014 & Steppe & & E.Medit. \\
\hline Bupleurum croceum Fenzl & 4079 & $\begin{array}{l}\text { B7 Tunceli: Mazgirt, between Tunceli and } \\
\text { Kovancılar, around of Kușcu }\end{array}$ & 867 & 02.06 .2014 & Steppe & Red. & Ir.-Tur. \\
\hline \multirow{4}{*}{ Bupleurum gerardii All. } & 5095 & $\begin{array}{l}\text { B7 Tunceli: Çemişgezek, } 2.5 \mathrm{~km} \text { from } \\
\text { Vişneli to Çemişgezek }\end{array}$ & 895 & 21.06 .2014 & \multirow{4}{*}{ Steppe } & \multirow{4}{*}{ Red. } & \multirow{4}{*}{ Unk. } \\
\hline & 4510 & B7 Tunceli: Mazgirt, 1 km W of Kayacı v. & 990 & 16.06 .2014 & & & \\
\hline & 5706 & $\begin{array}{l}\text { B7 Tunceli: Pertek, } 2 \text { km from Biçmekaya to } \\
\text { Koçpınar }\end{array}$ & 1323 & 25.07.2014 & & & \\
\hline & 4866 & $\begin{array}{l}\text { B8 Tunceli: Pülümür, } 4 \text { km from Kırklar to } \\
\text { Kızılmescit }\end{array}$ & 2450 & 18.06.2014 & & & \\
\hline \multirow{4}{*}{ Chaerophyllum crinitum Boiss. } & 4597 & $\begin{array}{l}\text { B7 Tunceli: Mazgirt, } 2 \mathrm{~km} \text { from Akdüven to } \\
\text { Güleç }\end{array}$ & 1125 & 16.06 .2014 & \multirow{4}{*}{ Steppe } & & \multirow{4}{*}{ Ir.-Tur. } \\
\hline & 4234 & $\begin{array}{l}\text { B7 Tunceli: Ovacık, } 15 \text { km from Ovacık- } \\
\text { Tunceli-Işıkvuran crossroads to Işıkvuran }\end{array}$ & 1670 & 04.06 .2014 & & & \\
\hline & 3761 & B7 Tunceli: Pertek, 1 km S of Beydamı v. & 1270 & 22.05 .2014 & & & \\
\hline & 4804 & B8 Tunceli: Pülümür, Kırklar v. & 1945 & 18.06 .2014 & & & \\
\hline $\begin{array}{l}\text { Chaerophyllum macrospermum } \\
\text { (Willd. ex Sprengel) Fisch. \& } \\
\text { C.A.Mey. ex Hohen }\end{array}$ & 5768 & $\begin{array}{l}\text { B7 Tunceli: Pülümür, } 16 \text { km from Tunceli to } \\
\text { Pülümür (Pülümür Valley) }\end{array}$ & 1240 & 06.08 .2014 & Steppe & & Unk. \\
\hline Daucus carota $\mathrm{L}$. & 5653 & $\begin{array}{l}\text { B7 Tunceli: Ovacık, } 2 \mathrm{~km} \text { from Ovacık } \\
\text { center to Gözeler v. }\end{array}$ & 1290 & 24.07 .2014 & $\begin{array}{l}\text { Field side, } \\
\text { steppe }\end{array}$ & Rcd. & Unk. \\
\hline Daucus guttatus Sibth. \& Sm. & 5698 & $\begin{array}{l}\text { B7 Tunceli: Center, between Çimenli and } \\
\text { Böğürtlen villages }\end{array}$ & 916 & 25.07 .2014 & $\begin{array}{l}\text { Field side, } \\
\text { steppe }\end{array}$ & Rcd. & Unk. \\
\hline \multirow{2}{*}{$\begin{array}{l}\text { Echinophora tenuifolia L. subsp. } \\
\text { sibthorpiana (Guss.) Tutin }\end{array}$} & 5699 & B7 Tunceli: Center, S of Çimenli village & 987 & 25.07 .2014 & \multirow{2}{*}{\begin{tabular}{|l|} 
Field side, \\
steppe
\end{tabular}} & \multirow{2}{*}{ Red. } & $\mathrm{Ir}$ Tur \\
\hline & 6131 & B7 Tunceli: Mazgirt, S of Dedebağ v. & 860 & 26.08 .2014 & & & Ir.- I ur. \\
\hline & 5153 & $\begin{array}{l}\text { B7 Tunceli: Çemişgezek, E of Anıl village } \\
\text { (Tahar Valley) }\end{array}$ & 1000 & 29.06 .2014 & & & \\
\hline & 4609 & $\begin{array}{l}\text { B7 Tunceli: Mazgirt, } 11 \mathrm{~km} \text { from Çukur to } \\
\text { Güleç }\end{array}$ & 1230 & 16.06 .2014 & & & \\
\hline $\begin{array}{l}\text { Eryngium campestre } \mathrm{L} . \text { var. } \\
\text { virens } \text { Link }\end{array}$ & 5292 & $\begin{array}{l}\text { B7 Tunceli: Center, } 14 \mathrm{~km} \text { from Tunceli to } \\
\text { Pülümür (Pülümür Valley) }\end{array}$ & 970 & 20.07 .2014 & Steppe & & Unk. \\
\hline & 5072 & $\begin{array}{l}\text { B7 Tunceli: Pertek, } 14 \mathrm{~km} \text { from Pertek to } \\
\text { Çemişgezek, } 2 \mathrm{~km} \text { after Singeç bridge, on the } \\
\text { right hills }\end{array}$ & 860 & 21.06 .2014 & & & \\
\hline & 5268 & B7 Tunceli: Pertek, Camikebir nh. & 1100 & 19.07 .2014 & & & \\
\hline Eryngium thyrsoideum Boiss. & 5770 & $\begin{array}{l}\text { B7 Tunceli: Pülümür, } 16 \mathrm{~km} \text { from Tunceli to } \\
\text { Pülümür (Pülümür Valley) }\end{array}$ & 1240 & 06.08 .2014 & Steppe & Rcd. & Ir.-Tur. \\
\hline Falcaria vulgaris Bernh. & 5566 & $\begin{array}{l}\text { B8 Tunceli: Pülümür, between Üçdam and } \\
\text { Erzurum-Erzincan mainroad }\end{array}$ & 1380 & 22.07 .2014 & Steppe & & Unk. \\
\hline Ferulago setifolia K.Koch & 4924 & $\begin{array}{l}\text { B7 Tunceli: Ovacık, } 8 \mathrm{~km} \text { from Işıkvuran to } \\
\text { Eskigedik }\end{array}$ & 2130 & 19.06.2014 & Steppe & & Ir.-Tur. \\
\hline
\end{tabular}




\begin{tabular}{|c|c|c|c|c|c|c|c|}
\hline Grammosciadium daucoides DC. & 4742 & $\begin{array}{l}\text { B7 Tunceli: Ovacık, } 11 \mathrm{~km} \text { from Ovacık to } \\
\text { Hozat, } 1 \mathrm{~km} \text { before junction of Bilgeç v. }\end{array}$ & 1820 & 17.06 .2014 & Steppe & & Ir.-Tur. \\
\hline \multirow{2}{*}{$\begin{array}{l}\text { Grammosciadium platycarpum } \\
\text { Boiss. \& Hausskn. }\end{array}$} & 4194 & $\begin{array}{l}\text { B7 Tunceli: Ovacık, } 1.5 \mathrm{~km} \text { from Ovacık- } \\
\text { Tunceli-Yakatarla crossroads to Yakatarla v. }\end{array}$ & 1320 & 04.06 .2014 & \multirow{2}{*}{ Steppe } & & \multirow{2}{*}{ Ir.-Tur. } \\
\hline & 5028 & $\begin{array}{l}\text { B7 Tunceli: Ovacık, } 10 \mathrm{~km} \text { from Eğripınar to } \\
\text { Yenikonak }\end{array}$ & 1570 & 20.06 .2014 & & & \\
\hline \multirow{2}{*}{$\begin{array}{l}\text { Heptaptera anisoptera (DC.) } \\
\text { Tutin }\end{array}$} & 4119 & $\begin{array}{l}\text { B7 Tunceli: Mazgirt, Akpazar, S of Yenice v. } \\
\text { (N of Şevki mezrası) }\end{array}$ & 1160 & 02.06 .2014 & \multirow[t]{2}{*}{ Steppe } & \multirow[t]{2}{*}{ Rcd. } & \multirow[t]{2}{*}{ Unk. } \\
\hline & 4532 & B7 Tunceli: Mazgirt, 1 km W of Kayacı v. & 990 & 16.06 .2014 & & & \\
\hline $\begin{array}{l}\text { Heracleum sphondylium L. } \\
\text { subsp. cyclocarpum } \text { (K.Koch) } \\
\text { P.H.Davis }\end{array}$ & 4986 & B7 Tunceli: Ovacık, W of Ziyaret v. & 1340 & 20.06 .2014 & Riverside & Rcd. & Euxine \\
\hline \multirow{2}{*}{$\begin{array}{l}\text { Heracleum trachyloma Fisch. \& } \\
\text { C.A.Mey. }\end{array}$} & 6002 & B7 Tunceli: Pülümür, NW of Közlüce v. & 1560 & 14.08 .2014 & \multirow[b]{2}{*}{ Wet places } & \multirow[b]{2}{*}{ Rcd. } & \multirow[b]{2}{*}{ Unk. } \\
\hline & 3957 & $\begin{array}{l}\text { B7 Tunceli: Center, } 5 \mathrm{~km} \text { from Tunceli to } \\
\text { Ovacık (Munzur Valley) }\end{array}$ & 940 & 25.05 .2014 & & & \\
\hline Johrenia dichotoma DC. & 5169 & $\begin{array}{l}\text { B7 Tunceli: Çemişgezek, Tahar valley (Ali } \\
\text { Boğazı) }\end{array}$ & 1024 & 29.06 .2014 & Steppe & Red. & Ir.-Tur. \\
\hline Laser trilobum (L.) Borkh. & 5259 & $\begin{array}{l}\text { B7 Tunceli: Nazımiye, Pülümür Valley, E } \\
\text { slopes of Hılbeşen bridge, across of Ağlayan } \\
\text { Kayalar }\end{array}$ & 1345 & 30.06 .2014 & Oak forest & & Unk. \\
\hline \multirow{2}{*}{$\begin{array}{l}\text { Lisaea strigosa (Banks \& Sol.) } \\
\text { Eig. }\end{array}$} & 4086 & $\begin{array}{l}\text { B7 Tunceli: Mazgirt, between Tunceli and } \\
\text { Kovancilar, around of Kuşcu }\end{array}$ & 867 & 02.06 .2014 & \multirow{2}{*}{$\begin{array}{l}\text { In gardens, } \\
\text { ruderal, } \\
\text { steppe }\end{array}$} & & \multirow[t]{2}{*}{ Ir.-Tur. } \\
\hline & 4546 & B7 Tunceli: Mazgirt, 1 km W of Kayacı v. & 985 & 16.06 .2014 & & & \\
\hline $\begin{array}{l}\text { Malabaila dasyantha (K.Koch) } \\
\text { Grossh. }\end{array}$ & 3850 & $\begin{array}{l}\text { B7 Tunceli: Hozat, } 2.5 \mathrm{~km} \text { from Demirkap1 to } \\
\text { Dervişcemal }\end{array}$ & 1700 & 23.05 .2014 & Steppe & Rcd. & Ir.-Tur. \\
\hline \multirow{2}{*}{ Malabaila secacul (Mill.) Boiss. } & 3906 & $\begin{array}{l}\text { B7 Tunceli: Pertek, } 12 \mathrm{~km} \text { from Pertek to } \\
\text { Çemişgezek, around of Singeç bridge }\end{array}$ & 855 & 24.05 .2014 & \multirow{2}{*}{ Steppe } & & \multirow{2}{*}{ Unk. } \\
\hline & 4782 & $\begin{array}{l}\text { B7 Tunceli: Pülümür, } 2 \text { km from Turnadere } \\
\text { to Közlüce }\end{array}$ & 1560 & 18.06 .2014 & & & \\
\hline Oenanthe sophiae Schisck. & 5744 & $\begin{array}{l}\text { B7 Tunceli: Mazgirt, } 3 \mathrm{~km} \text { from Akdüven to } \\
\text { Güleç }\end{array}$ & 1102 & 05.08 .2014 & Steppe & & Unk. \\
\hline \multirow{2}{*}{ Ormosciadium aucheri Boiss. } & 5105 & $\begin{array}{l}\text { B7 Tunceli: Çemişgezek, } 3 \mathrm{~km} \text { from } \\
\text { Arpaderen to Büyükörünce }\end{array}$ & 1112 & 21.06 .2014 & \multirow{2}{*}{ Steppe } & \multirow{2}{*}{ Rcd. } & \multirow{2}{*}{ Unk. } \\
\hline & 5305 & $\begin{array}{l}\text { B7 Tunceli: Nazımiye, } 3 \mathrm{~km} \text { from Tunceli- } \\
\text { Pülümür-Nazımiye crossroads to Nazımiye }\end{array}$ & 1190 & 20.07.2014 & & & \\
\hline \multirow{3}{*}{$\begin{array}{l}\text { Pastinaca sativa L. subsp. urens } \\
\text { (Req. ex Gren. \& Godr.) Čelak }\end{array}$} & 6113 & $\begin{array}{l}\text { B7 Tunceli: Pülümür, } 50 \mathrm{~km} \text { from Tunceli to } \\
\text { Pülümür (Pülümür Valley), Kırmızıköprü } \\
\text { Bridge }\end{array}$ & 1250 & 16.08 .2014 & \multirow{3}{*}{$\begin{array}{l}\text { Steppe, in } \\
\text { gardens }\end{array}$} & \multirow[t]{3}{*}{ Rcd. } & \multirow[t]{3}{*}{ Unk. } \\
\hline & 6069 & B8 Tunceli: Pülümür, Hasangazi v. & 1730 & 16.08 .2014 & & & \\
\hline & $\mathbf{5 5 3 7}$ & B8 Tunceli: Pülümür, Şampaşakaraderbent v. & 1912 & 22.07 .2014 & & & \\
\hline Peucedanum ruthenicum M.Bieb. & 5764 & $\begin{array}{l}\text { B7 Tunceli: Pülümür, } 45 \mathrm{~km} \text { from Tunceli to } \\
\text { Pülümür (Pülümür valley) }\end{array}$ & 1200 & 06.08 .2014 & Steppe & Rcd. & Unk. \\
\hline & 4907 & $\begin{array}{l}\text { B7 Tunceli: Pülümür, } 5 \text { km NW of Ardıçlı } \\
\text { (Gersunut) v. }\end{array}$ & 1975 & 18.06.2014 & & & \\
\hline Physocaulis nodosus (L.) Tausch & 4373 & B7 Tunceli: Pülümür, Kocatepe v. & 1680 & 05.06 .2014 & Steppe & Rcd. & Unk. \\
\hline & 3946 & $\begin{array}{l}\text { B7 Tunceli: Center, } 5 \mathrm{~km} \text { from Tunceli to } \\
\text { Ovacık (Munzur Valley) }\end{array}$ & 970 & 25.05 .2014 & & & \\
\hline & 5345 & B7 Tunceli: Nazımiye, E of Ramazanköy v. & 1440 & 20.07 .2014 & & & \\
\hline Pimpinella corymbosa Boiss. & 5671 & $\begin{array}{l}\text { B7 Tunceli: Ovacık, } 16 \mathrm{~km} \text { from Ovacık to } \\
\text { Tunceli (Munzur Valley) }\end{array}$ & 1180 & 24.07 .2014 & Steppe & & Ir.-Tur. \\
\hline Pimpinella kotschyana Boiss. & 5304 & $\begin{array}{l}\text { B7 Tunceli: Nazımiye, } 3 \mathrm{~km} \text { from Tunceli- } \\
\text { Pülümür-Nazımiye crossroads to Nazımiye }\end{array}$ & 1190 & 20.07 .2014 & Steppe & Rcd. & Ir.-Tur. \\
\hline Prangos corymbosa Boiss. & 4548 & B7 Tunceli: Mazgirt, 1 km W of Kayacı v. & 985 & 16.06 .2014 & Steppe & Red. & Ir.-Tur. \\
\hline & 4649 & $\begin{array}{l}\text { B7 Tunceli: Hozat, between Karacaköy and } \\
\text { Uzundal, the road of Sarisaltuk Türbesi }\end{array}$ & 1730 & 17.06 .2014 & & & \\
\hline Prangos ferulacea (L.) Lindl. & 4192 & $\begin{array}{l}\text { B7 Tunceli: Ovacık, } 1.5 \mathrm{~km} \text { from Ovacık- } \\
\text { Tunceli-Yakatarla crossroads to Yakatarla v. }\end{array}$ & 1320 & 04.06 .2014 & Steppe & & Unk. \\
\hline & 4468 & $\begin{array}{l}\text { B7 Tunceli: Hozat, } 3.5 \mathrm{~km} \text { from Hozat to } \\
\text { Pertek, junction of Kardelen v. }\end{array}$ & 1470 & 06.06 .2014 & Rocky & & \\
\hline Prangos peucedanifolia Fenzl & 3975 & $\begin{array}{l}\text { B7 Tunceli: Center, } 1 \text { km from Tunceli- } \\
\text { Ovacık-Dedeağaç crossroads to Dedeağaç v. } \\
\text { (Munzur Valley) }\end{array}$ & 1060 & 25.05 .2014 & $\begin{array}{l}\text { areas, } \\
\text { steppe }\end{array}$ & Rcd. & Ir.-Tur. \\
\hline Prangos platychlaena Boiss. & 5363 & B7 Tunceli: Nazımiye, 2 km E of Sarıyayla v. & 1945 & 20.07 .2014 & Steppe & * & Ir.-Tur. \\
\hline Scandix iberica M.Bieb. & 4050 & $\begin{array}{l}\text { B7 Tunceli: Ovacık, } 46 \mathrm{~km} \text { from Tunceli to } \\
\text { Ovacık, } 12 \mathrm{~km} \text { before Ovacık (Munzur } \\
\text { Valley) }\end{array}$ & 1213 & 26.05 .2014 & Steppe & & Unk. \\
\hline Smyrniopsis aucheri Boiss. & 4970 & $\begin{array}{l}\text { B7 Tunceli: Center, N of Sarıtaş v., Kalan } \\
\text { Valley }\end{array}$ & 1150 & 19.06.2014 & Steppe & & Ir.-Tur. \\
\hline & 5453 & B7 Tunceli: Hozat, S of Kurukaymak & 1495 & 21.07 .2014 & & & \\
\hline Smyrnium cordifolium Boiss. & 3937 & $\begin{array}{l}\text { B7 Tunceli: Center, } 2.5 \mathrm{~km} \text { from Tunceli to } \\
\text { Ovacık (Munzur Valley) }\end{array}$ & 930 & 25.05 .2014 & Steppe & & Ir.-Tur. \\
\hline Stenotaenia macrocarpa Freyn \& & 5890 & $\begin{array}{l}\text { B7 Tunceli: Nazımiye, } 11 \mathrm{~km} \text { NE of } \\
\text { Büyükyurt (Hakis) v. }\end{array}$ & 1938 & 07.08 .2014 & $\begin{array}{l}\text { Steppe } \\
\text { slopes, }\end{array}$ & & \\
\hline & 5546 & B8 Tunceli: Pülümür, 1-2 km E of Bağırpaşa, & 1916 & 22.07 .2014 & under oak & & \\
\hline
\end{tabular}




\begin{tabular}{|c|c|c|c|c|c|c|c|}
\hline & & N of Şampaşakaraderbent & & & $\begin{array}{l}\text { forest, } \\
\text { dried river } \\
\text { beds }\end{array}$ & & \\
\hline Torilis arvensis (Huds.) Link & 5683 & $\begin{array}{l}\text { B7 Tunceli: Center, } 26 \mathrm{~km} \text { from Tunceli to } \\
\text { Ovacık (Munzur Valley) }\end{array}$ & 1075 & 24.07.2014 & Steppe & Red. & Unk. \\
\hline Torilis leptophylla (L.) Rchb.f. & 4524 & B7 Tunceli: Mazgirt, 1 km W of Kayacı v. & 990 & 16.06 .2014 & Steppe & & Unk. \\
\hline $\begin{array}{l}\text { Trigonosciadium intermedium } \\
\text { Freyn \& Sint. ex Sint. }\end{array}$ & 4232 & $\begin{array}{l}\text { B7 Tunceli: Ovacık, } 15 \text { km from Ovacık- } \\
\text { Tunceli-Işıkvuran crossroads to Işıkvuran }\end{array}$ & 1670 & 04.06 .2014 & Steppe & $*$ & Ir.-Tur. \\
\hline \multirow[b]{2}{*}{ Turgenia latifolia (L.) Hoffm. } & 3738 & B7 Tunceli: Center, $1 \mathrm{~km} \mathrm{~S}$ of Aktuluk & 930 & 22.05 .2014 & \multirow[b]{2}{*}{ Steppe } & & \multirow[b]{2}{*}{ Unk. } \\
\hline & 4181 & $\begin{array}{l}\text { B7 Tunceli: Nazımiye, } 3 \mathrm{~km} \text { from Nazımiye } \\
\text { to Tunceli }\end{array}$ & 1510 & 03.06 .2014 & & & \\
\hline $\begin{array}{l}\text { Turgeniopsis foenicuIacea } \\
\text { (Fenzl) Boiss. }\end{array}$ & 4325 & $\begin{array}{l}\text { B7 Tunceli: Pülümür, between Kaymaztepe } \\
\text { and Kovuklu }\end{array}$ & 1545 & 05.06 .2014 & Steppe & Rcd. & Unk. \\
\hline Zosima absinthifolia (Vent.) Link & 3929 & B7 Tunceli: Center, Alibaba nh. & 930 & 25.05 .2014 & Steppe & & Unk. \\
\hline \multicolumn{8}{|l|}{ APOCYNACEAE Juss. } \\
\hline $\begin{array}{l}\text { Apocynum venetum L. subsp. } \\
\text { sarmatiense (Woodson) ined. }\end{array}$ & 5713 & B7 Tunceli: Center, N of Çimenli v. & 878 & 25.07 .2014 & Wet places & Red. & Unk. \\
\hline \multirow[b]{2}{*}{ Cynanchum acutum $\mathrm{L}}$. & 5703 & B7 Tunceli: Pertek, N of Pınarlar v. & 1213 & 25.07 .2014 & \multirow[b]{2}{*}{ Steppe } & & \multirow[b]{2}{*}{ Cosmp. } \\
\hline & 5755 & $\begin{array}{l}\text { B7 Tunceli: Pülümür, } 45 \mathrm{~km} \text { from Tunceli to } \\
\text { Pülümür (Pülümür valley) }\end{array}$ & 1200 & 06.08.2014 & & & \\
\hline $\begin{array}{l}\text { Vincetoxicum canescens (WiId.) } \\
\text { Decne. }\end{array}$ & 3820 & $\begin{array}{l}\text { B7 Tunceli: Center, Kocalar-Sütlüce-Tüllük } \\
\text { crossroads }\end{array}$ & 1115 & 23.05 .2014 & Steppe & & Unk. \\
\hline Vincetoxicum parviflorum Decne. & 4948 & $\begin{array}{l}\text { B7 Tunceli: Ovacık, N of Eskigedik v., } \\
\text { Munzur Mt. }\end{array}$ & 2160 & 19.06.2014 & $\begin{array}{l}\text { Rocky } \\
\text { areas, } \\
\text { steppe }\end{array}$ & $*$ & Ir.-Tur. \\
\hline $\begin{array}{l}\text { Vincetoxicum scandens Sommier } \\
\text { \& Levier }\end{array}$ & 5685 & $\begin{array}{l}\text { B7 Tunceli: Center, } 26 \mathrm{~km} \text { from Tunceli to } \\
\text { Ovacık (Munzur Valley) }\end{array}$ & 1075 & 24.07 .2014 & Steppe & & Unk. \\
\hline \multicolumn{8}{|l|}{ ARISTOLOCHIACEAE Juss. } \\
\hline $\begin{array}{l}\text { Aristolochia bottae Jaub. \& } \\
\text { Spach }\end{array}$ & 3823 & $\begin{array}{l}\text { B7 Tunceli: Center, between Tüllük and } \\
\text { Çıralı, Baldan-Tüllük junction }\end{array}$ & 1490 & 23.05.2014 & Steppe & Rcd. & Ir.-Tur. \\
\hline \multicolumn{8}{|l|}{ ASPARAGACEAE Juss. } \\
\hline $\begin{array}{l}\text { Ornithogalum plurifolium Y1ld. } \\
\text { \& K1lıç }\end{array}$ & 6501 & $\begin{array}{l}\text { B7 Tunceli: Center, } 1 \mathrm{~km} \text { from Sarıtaş to } \\
\text { Gözen (İksor), Kalan Valley }\end{array}$ & 1160 & 30.05 .2015 & Steppe & $*$ & Ir.-Tur. \\
\hline \multicolumn{8}{|l|}{ ASPLENIACEAE Newman } \\
\hline Asplenium ceterach $\mathrm{L}$. & 4954 & $\begin{array}{l}\text { B7 Tunceli: Ovacık, } \mathrm{N} \text { of Eskigedik v., } \\
\text { Munzur Mt. }\end{array}$ & 2160 & 19.06.2014 & $\begin{array}{l}\text { Rock } \\
\text { crevices }\end{array}$ & & Unk. \\
\hline $\begin{array}{l}\text { Asplenium haussknechtii God. \& } \\
\text { Reut. }\end{array}$ & 4456 & $\begin{array}{l}\text { B7 Tunceli: Hozat, between Demirkapı and } \\
\text { Dervişcemal, } 300 \mathrm{~m} \text { to Buzlupınar junction }\end{array}$ & 1810 & 06.06 .2014 & $\begin{array}{l}\text { Rocky } \\
\text { areas in } \\
\text { shadow }\end{array}$ & & Unk. \\
\hline \multicolumn{8}{|l|}{ ASTERACEAE Bercht. \& J.Presl } \\
\hline \multirow{3}{*}{$\begin{array}{l}\text { Achillea arabica Kotschy } \\
\text { (=Achillea biebersteinii } \\
\text { Afanasiev) }\end{array}$} & 3714 & $\begin{array}{l}\text { B7 Tunceli: Center, between Tunceli and } \\
\text { Pertek, junction of Kopuzlar v. }\end{array}$ & 1020 & 22.05 .2014 & \multirow{3}{*}{ Steppe } & & \multirow{3}{*}{ Ir.-Tur. } \\
\hline & 3759 & B7 Tunceli: Pertek, 1 km S of Beydamı v. & 1270 & 22.05 .2014 & & & \\
\hline & 4416 & $\begin{array}{l}\text { B7 Tunceli: Pülümür, between Dereboyu and } \\
\text { Sağlamtaş }\end{array}$ & 1540 & 05.06 .2014 & & & \\
\hline Achillea coarctata Poir. & 4038 & $\begin{array}{l}\text { B7 Tunceli: Ovacık, } 41 \mathrm{~km} \text { from Tunceli to } \\
\text { Ovacık (Munzur Valley) }\end{array}$ & 1175 & 26.05.2014 & Steppe & & Unk. \\
\hline Achillea millefolium $\mathrm{L}$. & 6009 & $\begin{array}{l}\text { B7 Tunceli: Pülümür, between } \\
\text { Boğazkaraderbent and Hasangazi }\end{array}$ & 1800 & 14.08.2014 & Steppe & & $\begin{array}{l}\text { Euro.- } \\
\text { Sib. }\end{array}$ \\
\hline $\begin{array}{l}\text { Achillea nobilis L. subsp. } \\
\text { neilreichii (A.Kern.) Velen. }\end{array}$ & 5482 & $\begin{array}{l}\text { B8 Tunceli: Pülümür, between Derindere and } \\
\text { Kırdım }\end{array}$ & 1905 & 22.07.2014 & Steppe & & $\begin{array}{l}\text { Euro.- } \\
\text { Sib. } \\
\end{array}$ \\
\hline $\begin{array}{l}\text { Achillea pseudoaleppica } \\
\text { Hausskn. ex Hub.-Mor. }\end{array}$ & 3979 & $\begin{array}{l}\text { B7 Tunceli: Center, } 1 \mathrm{~km} \text { from Tunceli- } \\
\text { Ovacık-Dedeağaç crossroads to Dedeağaç v. } \\
\text { (Munzur Valley) }\end{array}$ & 1060 & 25.05 .2014 & $\begin{array}{l}\text { degraded } \\
\text { oaks forest }\end{array}$ & $\begin{array}{l}* \\
\text { Rcd. }\end{array}$ & Ir.-Tur. \\
\hline Achillea schischkinii Sosn. & 4445 & $\begin{array}{l}\text { B7 Tunceli: Hozat, between Demirkapı and } \\
\text { Dervişcemal, } 300 \mathrm{~m} \text { to Buzlupınar junction }\end{array}$ & 1810 & 06.06 .2014 & Steppe & $*$ & Ir.-Tur. \\
\hline \multirow{3}{*}{$\begin{array}{l}\text { Anthemis cretica L. subsp. } \\
\text { pontica (Willd.) Grierson }\end{array}$} & 4470 & $\begin{array}{l}\text { B7 Tunceli: Hozat, } 3.5 \mathrm{~km} \text { from Hozat to } \\
\text { Pertek, junction of Kardelen v. }\end{array}$ & 1470 & 06.06 .2014 & \multirow{3}{*}{$\begin{array}{l}\text { Eroded } \\
\text { slopes, } \\
\text { steppe }\end{array}$} & & \multirow{3}{*}{ Unk. } \\
\hline & 4135 & $\begin{array}{l}\text { B7 Tunceli: Mazgirt, between Tunceli and } \\
\text { Kovancilar, junction of Kizılc1k v. }\end{array}$ & 1060 & 02.06 .2014 & & & \\
\hline & 3961 & $\begin{array}{l}\text { B7 Tunceli: Center, } 7 \mathrm{~km} \text { from Tunceli to } \\
\text { Ovacık (Munzur Valley) }\end{array}$ & 960 & 25.05 .2014 & & & \\
\hline \multirow{2}{*}{ Artemisia absinthium L. } & 5848 & $\begin{array}{l}\text { B7 Tunceli: Nazımiye, } 4 \text { km NE of } \\
\text { Büyükyurt (Hakis) v. }\end{array}$ & 1775 & 07.08 .2014 & \multirow{2}{*}{ Steppe } & & \multirow{2}{*}{ Unk. } \\
\hline & 5374 & $\begin{array}{l}\text { B7 Tunceli: Nazımiye, } 2.5 \mathrm{~km} \text { E of Sarıyayla } \\
\text { v. }\end{array}$ & 2000 & 20.07.2014 & & & \\
\hline Artemisia marschalliana Spreng. & 5567 & $\begin{array}{l}\text { B8 Tunceli: Pülümür, between Üçdam and } \\
\text { Erzurum-Erzincan mainroad }\end{array}$ & 1380 & 22.07 .2014 & steppe & Rcd. & Unk. \\
\hline Carduus lanuginosus Willd. & 5826 & $\begin{array}{l}\text { B7 Tunceli: Ovacık, } 1 \text { km NE of Buyer Baba } \\
\text { Lake }\end{array}$ & 2820 & 06.08 .2014 & Mt. steppe & $*$ & Unk. \\
\hline $\begin{array}{l}\text { Carduus pycnocephalus L. subsp. } \\
\text { albidus (M.Bieb.) Kazmi }\end{array}$ & 4081 & $\begin{array}{l}\text { B7 Tunceli: Mazgirt, between Tunceli and } \\
\text { Kovancılar, around of Kuşcu }\end{array}$ & 867 & 02.06 .2014 & Steppe & & Unk. \\
\hline
\end{tabular}




\begin{tabular}{|c|c|c|c|c|c|c|c|}
\hline $\begin{array}{l}\text { Carlina oligocephala Boiss. \& } \\
\text { Kotschy }\end{array}$ & 5423 & $\begin{array}{l}\text { B7 Tunceli: Hozat, between Karaçavuş and } \\
\text { Boydaş }\end{array}$ & 1720 & 21.07.2014 & Steppe & Red. & Unk. \\
\hline $\begin{array}{l}\text { Centaurea aggregata Fisch. \& } \\
\text { C.A.Mey. ex DC. }\end{array}$ & 5358 & B7 Tunceli: Nazımiye, Sarıyayla v. & 1835 & 20.07.2014 & Steppe & & Unk. \\
\hline \multirow{3}{*}{ Centaurea balsamita Lam. } & 5434 & B7 Tunceli: Hozat, W of Boydaş v. & 1430 & 21.07 .2014 & \multirow{3}{*}{$\begin{array}{l}\text { Steppe, } \\
\text { field side, } \\
\text { ruderal, } \\
\text { degraded } \\
\text { oak forest }\end{array}$} & \multirow{3}{*}{ Rcd. } & \multirow{3}{*}{ Ir.-Tur. } \\
\hline & 4089 & $\begin{array}{l}\text { B7 Tunceli: Mazgirt, between Tunceli and } \\
\text { Kovancilar, around of Kuşcu }\end{array}$ & 867 & 02.06 .2014 & & & \\
\hline & 5718 & $\begin{array}{l}\text { B7 Tunceli: Mazgirt, } 4.5 \mathrm{~km} \text { from Kızılcık to } \\
\text { Kuşcu }\end{array}$ & 1000 & 25.07 .2014 & & & \\
\hline \multirow{5}{*}{$\begin{array}{l}\text { Centaurea carduiformis DC. var. } \\
\text { carduiformis }\end{array}$} & 5212 & $\begin{array}{l}\text { B7 Tunceli, Çemişgezek, } 1 \text { km NE of Alakuş } \\
\text { village }\end{array}$ & 1390 & 29.06 .2014 & \multirow{5}{*}{$\begin{array}{l}\text { Steppe, } \\
\text { field side, } \\
\text { ruderal, } \\
\text { degraded } \\
\text { oak forest }\end{array}$} & \multirow{5}{*}{$*$} & \multirow{5}{*}{ Unk. } \\
\hline & 5187 & $\begin{array}{l}\text { B7 Tunceli: Çemişgezek, } 5.5 \mathrm{~km} \text { from } \\
\text { Çemişgezek to Alakuş }\end{array}$ & 1296 & 29.06.2014 & & & \\
\hline & 5433 & B7 Tunceli: Hozat, W of Boydaş v. & 1430 & 21.07 .2014 & & & \\
\hline & 5641 & $\begin{array}{l}\text { B7 Tunceli: Ovacık, } 30 \mathrm{~km} \text { from Tunceli to } \\
\text { Ovacık (Munzur Valley) }\end{array}$ & 1070 & 24.07.2014 & & & \\
\hline & 4112 & $\begin{array}{l}\text { B7 Tunceli: Mazgirt, } 7 \mathrm{~km} \text { from Kizılcık to } \\
\text { Göktepe }\end{array}$ & 935 & 02.06 .2014 & & & \\
\hline $\begin{array}{l}\text { Centaurea carduiformis DC. var. } \\
\text { thrinciifolia (DC.) Wagenitz }\end{array}$ & 4784 & $\begin{array}{l}\text { B7 Tunceli: Pülümür, } 2 \mathrm{~km} \text { from Turnadere } \\
\text { to Közlüce }\end{array}$ & 1560 & 18.06.2014 & Steppe & Red. & Unk. \\
\hline \multirow{5}{*}{ Centaurea consanguinea DC. } & 4490 & $\begin{array}{l}\text { B7 Tunceli: Çemişgezek, } 2 \mathrm{~km} \text { from } \\
\text { Bulgurtepe to Payamdüzü }\end{array}$ & 1060 & 06.06 .2014 & \multirow{5}{*}{ Steppe } & \multirow{5}{*}{$*$} & \multirow{5}{*}{ Ir.-Tur. } \\
\hline & 5462 & B7 Tunceli: Hozat, $1 \mathrm{~km}$ E of Uzundal v. & 1900 & 21.07 .2014 & & & \\
\hline & 4562 & $\begin{array}{l}\text { B7 Tunceli: Mazgirt, } 1 \mathrm{~km} \text { from Ortaharman } \\
\text { to Sülüntaş }\end{array}$ & 1220 & 16.06.2014 & & & \\
\hline & 5967 & $\begin{array}{l}\text { B7 Tunceli: Pertek, } 9 \mathrm{~km} \text { from Pertek to } \\
\text { Çemişgezek, } 2.5 \mathrm{~km} \text { before Singeç bridge }\end{array}$ & 875 & 13.08.2014 & & & \\
\hline & 5283 & $\begin{array}{l}\text { B7 Tunceli: Pertek, } 8 \mathrm{~km} \text { from Pertek to } \\
\text { Tunceli }\end{array}$ & 1300 & 19.07.2014 & & & \\
\hline \multirow[t]{2}{*}{ Centaurea derderiifolia Wagenitz } & 5110 & $\begin{array}{l}\text { B7 Tunceli: Pertek, } 3 \mathrm{~km} \text { from Elazı ̆g-Pertek- } \\
\text { Çemişgezek crossroads to Çemişgezek (1 km } \\
\text { S of Çorovan v.) }\end{array}$ & 870 & 28.06 .2014 & \multirow[t]{2}{*}{ Steppe } & \multirow[t]{2}{*}{$*$} & \multirow[t]{2}{*}{ Ir.-Tur. } \\
\hline & 5289 & B7 Tunceli: Pertek, 1 km E of Mercimek v. & 1420 & 19.07.2014 & & & \\
\hline Centaurea fenzlii Reichardt & 5432 & B7 Tunceli: Hozat, W of Boydaş v. & 1430 & 21.07.2014 & $\begin{array}{l}\text { degraded } \\
\text { oaks forest }\end{array}$ & $*$ & Ir.-Tur. \\
\hline \multirow{3}{*}{ Centaurea glastifolia $\mathrm{L}$. } & 5359 & B7 Tunceli: Nazımiye, Sarıyayla v. & 1835 & 20.07 .2014 & \multirow{3}{*}{ Steppe } & \multirow{3}{*}{ Red. } & \multirow{3}{*}{ Ir.-Tur. } \\
\hline & 6011 & $\begin{array}{l}\text { B8 Tunceli: Pülümür, } 4 \text { km from Hasangazi } \\
\text { to Balpayam }\end{array}$ & 1832 & 14.08.2014 & & & \\
\hline & 5503 & B8 Tunceli: Pülümür, Kırdım v. & 2080 & 22.07 .2014 & & & \\
\hline $\begin{array}{l}\text { Centaurea iberica } \text { Trev. ex } \\
\text { Sprengel }\end{array}$ & 5296 & $\begin{array}{l}\text { B7 Tunceli: Center, } 14 \mathrm{~km} \text { from Tunceli to } \\
\text { Pülümür (Pülümür Valley) }\end{array}$ & 970 & 20.07.2014 & $\begin{array}{l}\text { Ruderal, } \\
\text { steppe }\end{array}$ & & Unk. \\
\hline \multirow[b]{2}{*}{ Centaurea kurdica Reichardt } & 5133 & B7 Tunceli: Pertek, 2 km N of Geçityaka v. & 960 & 28.06 .2014 & \multirow{2}{*}{$\begin{array}{l}\text { Steppe, } \\
\text { field side, } \\
\text { ruderal, } \\
\text { degraded } \\
\text { oak forest }\end{array}$} & \multirow[b]{2}{*}{$*$} & \multirow[b]{2}{*}{ Ir.-Tur. } \\
\hline & 5213 & B7 Tunceli: Pertek, E of Yeniköy v. & 1360 & 29.06 .2014 & & & \\
\hline $\begin{array}{l}\text { Centaurea polypodiifolia Boiss. } \\
\text { var. polypodiifolia }\end{array}$ & 4519 & B7 Tunceli: Mazgirt, 1 km W of Kayacı v. & 990 & 16.06.2014 & Steppe & & Unk. \\
\hline $\begin{array}{l}\text { Centaurea polypodiifolia } \text { Boiss. } \\
\text { var. pseudobehen (Boiss.) } \\
\text { Wagenitz }\end{array}$ & 5435 & B7 Tunceli: Hozat, W of Boydaş v. & 1430 & 21.07.2014 & Steppe & & Ir.-Tur. \\
\hline $\begin{array}{l}\text { Centaurea pseudoscabiosa Boiss. } \\
\& \text { Buhse subsp. araratica (Azn.) } \\
\text { Wagenitz }\end{array}$ & 6072 & $\begin{array}{l}\text { B8 Tunceli: Pülümür, } 3 \text { km from Hasangazi } \\
\text { to Kırklar }\end{array}$ & 1890 & 16.08.2014 & Steppe & Rcd. & Ir.-Tur. \\
\hline $\begin{array}{l}\text { Centaurea pseudoscabiosa Boiss. } \\
\text { \& Buhse subsp. pseudoscabiosa }\end{array}$ & 5528 & B8 Tunceli: Pülümür, Şampaşakaraderbent v. & 1820 & 22.07 .2014 & Steppe & & Unk. \\
\hline \multirow{2}{*}{ Centaurea pterocaula Trautv. } & 6207 & $\begin{array}{l}\text { B7 Tunceli: Hozat, } 18 \mathrm{~km} \text { from Hozat to } \\
\text { Ovacik }\end{array}$ & 1967 & 28.08.2014 & \multirow{2}{*}{ Steppe } & Rcd & \\
\hline & 6079 & $\begin{array}{l}\text { B8 Tunceli: Pülümür, } 3 \text { km from Hasangazi } \\
\text { to Kırklar }\end{array}$ & 1890 & 16.08 .2014 & & Rcd. & Ir.-Tur. \\
\hline Centaurea rhizantha C.A.Mey. & 5815 & $\begin{array}{l}\text { B7 Tunceli: Ovacık, } 8 \mathrm{~km} \text { from Sarigül to } \\
\text { Yalmanlar, the walk way of Buyer Baba } \\
\text { sanctuary }\end{array}$ & 2680 & 06.08 .2014 & Steppe & & Ir.-Tur. \\
\hline & 6661 & $\begin{array}{l}\text { B7 Tunceli: Pülümür, } 11 \text { km NW of Ardıçlı } \\
\text { (Gersunut) v. (Munzur Mt.) }\end{array}$ & 2516 & 27.06.2015 & & & \\
\hline & 5099 & $\begin{array}{l}\text { B7 Tunceli: Çemişgezek, } 2 \mathrm{~km} \text { from Vişneli } \\
\text { to Arpaderen }\end{array}$ & 1080 & 21.06 .2014 & & & \\
\hline Centaurea rigida Banks \& Sol. & 5116 & $\begin{array}{l}\text { B7 Tunceli: Pertek, } 3 \mathrm{~km} \text { from Elazı ̆̆-Pertek- } \\
\text { Çemişgezek crossroads to Çemişgezek (1 km } \\
\text { S of Çorovan v.) }\end{array}$ & 870 & 28.06 .2014 & $\begin{array}{l}\text { Field side, } \\
\text { steppe }\end{array}$ & Red. & Ir.-Tur. \\
\hline & 5605 & B7 Tunceli: Nazımiye, 6 km E of Ayranlı v. & 1800 & 23.07 .2014 & & & \\
\hline $\begin{array}{l}\text { Centaurea saligna (K.Koch) } \\
\text { Wagenitz }\end{array}$ & 5665 & $\begin{array}{l}\text { B7 Tunceli: Ovacık, } 12 \mathrm{~km} \text { from Eğripınar to } \\
\text { Yenikonak }\end{array}$ & 1570 & 24.07 .2014 & Steppe & $*$ & Ir.-Tur. \\
\hline & 5897 & B7 Tunceli: Ovacık, Tunceli-Ovacık- & 1240 & 08.08 .2014 & & & \\
\hline
\end{tabular}




\begin{tabular}{|c|c|c|c|c|c|c|c|}
\hline & & Yakatarla junction & & & & & \\
\hline & 5562 & $\begin{array}{l}\text { B8 Tunceli: Pülümür, } 3 \mathrm{~km} \text { from Bardakçı to } \\
\text { Derindere }\end{array}$ & 1740 & 22.07 .2014 & & & \\
\hline & 5498 & B8 Tunceli: Pülümür, W of Kırdım v. & 2070 & 22.07 .2014 & & & \\
\hline Centaurea solstitialis L. & 5298 & $\begin{array}{l}\text { B7 Tunceli: Center, } 14 \mathrm{~km} \text { from Tunceli to } \\
\text { Pülümür (Pülümür Valley) }\end{array}$ & 970 & 20.07 .2014 & $\begin{array}{l}\text { Ruderal, } \\
\text { steppe }\end{array}$ & & Unk. \\
\hline \multirow{5}{*}{$\begin{array}{l}\text { Centaurea spectabilis (DC.) } \\
\text { Sch.Bip. var. microlopha (Boiss.) } \\
\text { Wagenitz }\end{array}$} & 4578 & B7 Tunceli: Mazgirt, 1 km S of Geçitveren v. & 1345 & 16.06 .2014 & \multirow{5}{*}{ Steppe } & & \multirow{5}{*}{ Ir.-Tur. } \\
\hline & 5872 & $\begin{array}{l}\text { B7 Tunceli: Nazımiye, } 6 \mathrm{~km} \mathrm{NE} \text { of } \\
\text { Büyükyurt (Hakis) v. }\end{array}$ & 2015 & 07.08.2014 & & & \\
\hline & 5032 & B7 Tunceli: Ovacık, 2 km N of Yenikonak v. & 1705 & 20.06 .2014 & & & \\
\hline & 5064 & $\begin{array}{l}\text { B7 Tunceli: Pertek, } 17 \mathrm{~km} \text { from Pertek to } \\
\text { Tunceli }\end{array}$ & 1300 & 21.06 .2014 & & & \\
\hline & 5534 & B8 Tunceli: Pülümür, Şampaşakaraderbent v. & 1912 & 22.07 .2014 & & & \\
\hline \multirow{2}{*}{$\begin{array}{l}\text { Centaurea urvillei DC. subsp. } \\
\text { hayekiana Wagenitz }\end{array}$} & $\mathbf{5 0 7 0}$ & $\begin{array}{l}\text { B7 Tunceli: Pertek, } 4.5 \mathrm{~km} \text { from Pertek to } \\
\text { Tunceli, S of Mercimek v. }\end{array}$ & 1307 & 21.06 .2014 & \multirow{2}{*}{$\begin{array}{l}\text { On rock, } \\
\text { steppe }\end{array}$} & & \multirow{2}{*}{ Ir.-Tur. } \\
\hline & 4010 & $\begin{array}{l}\text { B7 Tunceli: Center, } 15 \mathrm{~km} \text { from Tunceli to } \\
\text { Ovacık (Munzur Valley) }\end{array}$ & 1010 & 25.05 .2014 & & & \\
\hline $\begin{array}{l}\text { Centaurea urvillei DC. subsp. } \\
\text { nimrodis (Boiss. \& Hausskn.) } \\
\text { Wagenitz }\end{array}$ & 5058 & $\begin{array}{l}\text { B7 Tunceli: Ovacık, between Yarımkaya and } \\
\text { Şahverdi }\end{array}$ & 1520 & 20.06 .2014 & Steppe & Rcd. & Ir.-Tur. \\
\hline Centaurea virgata Lam. & 5299 & $\begin{array}{l}\text { B7 Tunceli: Center, } 14 \mathrm{~km} \text { from Tunceli to } \\
\text { Pülümür (Pülümür Valley) }\end{array}$ & 970 & 20.07.2014 & $\begin{array}{l}\text { Steppe, } \\
\text { field side, } \\
\text { ruderal, } \\
\text { degraded } \\
\text { oak forest }\end{array}$ & & Ir.-Tur. \\
\hline Chardinia orientalis (L.) Kuntze & 4426 & $\begin{array}{l}\text { B7 Tunceli: Pülümür, } 9 \mathrm{~km} \text { from Pülümür to } \\
\text { Tunceli (Pülümür valley), S of Kangallı v. }\end{array}$ & 1324 & 05.06 .2014 & Steppe & & Ir.-Tur. \\
\hline \multirow{2}{*}{ Cichorium intybus $\mathrm{L}$. } & Obs. & $\begin{array}{l}\text { B7 Tunceli: Mazgirt, } 5.5 \mathrm{~km} \text { from Kale to } \\
\text { Doludizgin }\end{array}$ & 1560 & 15.08 .2014 & \multirow{2}{*}{$\begin{array}{l}\text { Steppe, } \\
\text { degraded } \\
\text { oaks forest }\end{array}$} & & \multirow{2}{*}{ Unk. } \\
\hline & Obs. & $\begin{array}{l}\text { B7 Tunceli: Center, } 3.5 \mathrm{~km} \text { from Aktuluk to } \\
\text { Buğulu }\end{array}$ & 1065 & 06.06 .2014 & & & \\
\hline \multirow{3}{*}{ Cirsium aristatum DC. } & 5721 & B7 Tunceli: Mazgirt, NE of Akdüven v. & 1040 & 05.08 .2014 & \multirow{3}{*}{ Steppe } & \multirow{3}{*}{$\begin{array}{l}* \\
\text { Rcd. }\end{array}$} & \multirow{3}{*}{ Ir.-Tur. } \\
\hline & 6271 & B7 Tunceli: Mazgirt, W of Yenice v. & 1140 & 19.09 .2014 & & & \\
\hline & 6272 & B7 Tunceli: Mazgirt, W of Yenice v. & 1140 & 19.09 .2014 & & & \\
\hline \multirow[t]{2}{*}{ Cirsium arvense (L.) Scop. } & 5985 & $\begin{array}{l}\text { B7 Tunceli: Nazımiye, } 10 \mathrm{~km} \text { NE of } \\
\text { Büyükyurt (Hakis) v. }\end{array}$ & 1940 & 14.08 .2014 & \multirow[t]{2}{*}{ Wet places } & & \multirow[t]{2}{*}{ Unk. } \\
\hline & 5362 & B7 Tunceli: Nazımiye, Sarıyayla v. & 1835 & 20.07 .2014 & & & \\
\hline \multirow{2}{*}{ Cirsium cephalotes Boiss. } & 5989 & $\begin{array}{l}\text { B7 Tunceli: Nazımiye, } 8 \mathrm{~km} \mathrm{NE} \text { of } \\
\text { Büyükyurt (Hakis) v. }\end{array}$ & 2040 & 14.08 .2014 & \multirow{2}{*}{ Steppe } & \multirow{2}{*}{ Red. } & \multirow{2}{*}{ Euxine } \\
\hline & 5795 & $\begin{array}{l}\text { B7 Tunceli: Pülümür, between Eğimli and } \\
\text { Kocatepe }\end{array}$ & 2341 & 06.08 .2014 & & & \\
\hline \multirow[t]{2}{*}{ Cirsium haussknechtii Boiss. } & 6114 & $\begin{array}{l}\text { B7 Tunceli: Center, } 3 \mathrm{~km} \text { from Çılga v. to } \\
\text { Tunceli center }\end{array}$ & 1480 & 17.08 .2014 & \multirow[t]{2}{*}{ Steppe } & & \multirow[t]{2}{*}{ Ir.-Tur. } \\
\hline & 5984 & B7 Tunceli: Hozat, N of Çığırlı v. & 1660 & 13.08 .2014 & & & \\
\hline $\begin{array}{l}\text { Cirsium leucocephalum (Willd.) } \\
\text { Spreng. }\end{array}$ & 6074 & $\begin{array}{l}\text { B8 Tunceli: Pülümür, } 3 \mathrm{~km} \text { from Hasangazi } \\
\text { to Kırklar }\end{array}$ & 1890 & 16.08 .2014 & Steppe & & Ir.-Tur. \\
\hline $\begin{array}{l}\text { Cirsium leuconeurum Boiss. \& } \\
\text { Hausskn. }\end{array}$ & 6365 & $\begin{array}{l}\text { B7 Tunceli: Pertek, between Kayabağ and } \\
\text { Derebucağ1 }\end{array}$ & 1655 & 22.09 .2014 & Steppe & $\begin{array}{l}* \\
\text { Red. }\end{array}$ & Medit. \\
\hline $\begin{array}{l}\text { Cirsium simplex C.A.Mey. subsp. } \\
\text { armenum (DC.) Petr. }\end{array}$ & 5832 & $\begin{array}{l}\text { B7 Tunceli: Ovacık, } 800 \mathrm{~m} \mathrm{NE} \text { of Buyer } \\
\text { Baba Lake }\end{array}$ & 2890 & 06.08 .2014 & $\begin{array}{l}\text { Mt. } \\
\text { pastures }\end{array}$ & Red. & Unk. \\
\hline $\begin{array}{l}\text { Cirsium simplex C.A.Mey. subsp. } \\
\text { simplex }\end{array}$ & 5793 & $\begin{array}{l}\text { B7 Tunceli: Pülümür, between Eğimli and } \\
\text { Kocatepe }\end{array}$ & 2341 & 06.08 .2014 & Wet places & & $\begin{array}{l}\text { Euxine } \\
\text { (Mt.) }\end{array}$ \\
\hline Cirsium sommieri Petr. & 6251 & B7 Tunceli: Center, Alacık road & 1330 & 05.09 .2014 & Oak forest & $*$ & Ir.-Tur. \\
\hline $\begin{array}{l}\text { Cirsium subinerme Fisch. \& } \\
\text { C.A.Mey. }\end{array}$ & 6016 & $\begin{array}{l}\text { B8 Tunceli: Pülümür, } 2 \mathrm{~km} \text { from Balpayam } \\
\text { to Ağaşenliği }\end{array}$ & 1830 & 14.08 .2014 & Wet places & & Ir.-Tur. \\
\hline \multirow{4}{*}{ Cirsium vulgare (Savi) Ten. } & 6200 & $\begin{array}{l}\text { B7 Tunceli: Hozat, between Uzundal and } \\
\text { Sarısaltuk }\end{array}$ & 1700 & 28.08 .2014 & \multirow{4}{*}{ Steppe } & & \\
\hline & 6201 & $\begin{array}{l}\text { B7 Tunceli: Hozat, between Uzundal and } \\
\text { Sarısaltuk }\end{array}$ & 1790 & 28.08 .2014 & & Rcd. & Unk. \\
\hline & 5746 & B7 Tunceli: Mazgirt, SW of Güleç v. & 1105 & 05.08 .2014 & & & \\
\hline & 6191 & $\begin{array}{l}\text { B7 Tunceli: Center, } 6 \text { km from Gökçek to } \\
\text { Çıralı }\end{array}$ & 1560 & 27.08 .2014 & & & \\
\hline $\begin{array}{l}\text { Cirsium yildizianum Arabacı \& } \\
\text { Dirmenci }\end{array}$ & 6220 & $\begin{array}{l}\text { B7 Tunceli: Nazımiye, between Güneycik } \\
\text { and Beytașı }\end{array}$ & 1665 & 01.09 .2014 & Steppe & $\begin{array}{l}* \\
\text { Rcd. }\end{array}$ & Ir.-Tur. \\
\hline Cnicus benedictus $\mathrm{L}$. & 3898 & $\begin{array}{l}\text { B7 Tunceli: Pertek, } 9 \mathrm{~km} \text { from Pertek to } \\
\text { Çemişgezek, } 2.5 \mathrm{~km} \text { before Singeç bridge }\end{array}$ & 865 & 24.05 .2014 & Steppe & & Unk. \\
\hline & 3793 & $\begin{array}{l}\text { B7 Tunceli: Center, between Tunceli and } \\
\text { Sütlüce, } 1.8 \mathrm{~km} \text { from Sütlüce junction } \\
\end{array}$ & 1030 & 23.05 .2014 & Field side, & & I \\
\hline Cota altissima (L.) J.Gay & 3878 & $\begin{array}{l}\text { B7 Tunceli: Pertek, } 5 \mathrm{~km} \text { from Pertek to } \\
\text { Çemişgezek }\end{array}$ & 866 & 24.05 .2014 & steppe & & Unk. \\
\hline Cota austriaca (Jacq.) Sch.Bip. & 4071 & $\begin{array}{l}\text { B7 Tunceli: Mazgirt, between Tunceli and } \\
\text { Kovancılar, around of Kuşcu }\end{array}$ & 867 & 02.06 .2014 & Field side, & & Unk. \\
\hline & 8099a & B7 Tunceli: Pertek, Kaledibi nh. & 895 & 01.05 .2018 & & & \\
\hline
\end{tabular}




\begin{tabular}{|c|c|c|c|c|c|c|c|}
\hline $\begin{array}{l}\text { Cota melanoloma (Trauvt.) } \\
\text { Holub }\end{array}$ & 4340 & $\begin{array}{l}\text { B7 Tunceli: Pülümür, between Sarıül and } \\
\text { Kocatepe }\end{array}$ & 1900 & 05.06 .2014 & Steppe & & Ir.-Tur. \\
\hline \multirow{5}{*}{$\begin{array}{l}\text { Cota tinctoria (L.) J.Gay ex } \\
\text { Guss. }\end{array}$} & 3695 & $\begin{array}{l}\text { B7 Tunceli: Center, Tunceli - Elazı̆g road, } \\
700 \text { m after Aktuluk v. }\end{array}$ & 930 & 22.05 .2014 & \multirow{5}{*}{$\begin{array}{l}\text { Steppe, } \\
\text { degraded } \\
\text { oak forest }\end{array}$} & & \multirow{5}{*}{ Unk. } \\
\hline & 4218 & $\begin{array}{l}\text { B7 Tunceli: Ovacık, } 5 \mathrm{~km} \text { from Ovacık- } \\
\text { Tunceli-Yakatarla to Yakatarla }\end{array}$ & 1620 & 04.06 .2014 & & & \\
\hline & 4321 & $\begin{array}{l}\text { B7 Tunceli: Pülümür, between Kaymaztepe } \\
\text { and Kovuklu }\end{array}$ & 1545 & 05.06 .2014 & & & \\
\hline & 3963 & $\begin{array}{l}\text { B7 Tunceli: Center, } 2.5 \mathrm{~km} \text { from Tunceli- } \\
\text { Ovacık-Dedeağaç crossroads to Dedeağaç v. } \\
\text { (Munzur Valley) }\end{array}$ & 1100 & 25.05 .2014 & & & \\
\hline & 4099 & $\begin{array}{l}\text { B7 Tunceli: Mazgirt, } 7 \mathrm{~km} \text { from Kızılcık to } \\
\text { Göktepe }\end{array}$ & 935 & 02.06 .2014 & & & \\
\hline $\begin{array}{l}\text { Cota wiedemanniana (Fisch. \& } \\
\text { C.A.Mey.) Holub }\end{array}$ & 4495 & $\begin{array}{l}\text { B7 Tunceli: Pertek, between Çemişgezek and } \\
\text { Elazığ, } 6 \mathrm{~km} \text { from Bulgurtepe to Payamdüzü }\end{array}$ & 980 & 06.06 .2014 & Steppe & & Unk. \\
\hline $\begin{array}{l}\text { Cousinia eriocephala Boiss. \& } \\
\text { Hausskn. }\end{array}$ & 5796 & $\begin{array}{l}\text { B7 Tunceli: Ovacik, } 9 \mathrm{~km} \text { from Kocatepe to } \\
\text { Yalmanlar }\end{array}$ & 2510 & 06.08 .2014 & Steppe & $*$ & Ir.-Tur. \\
\hline \multirow[b]{2}{*}{ Cousinia intertexta Freyn \& Sint. } & 5100 & $\begin{array}{l}\text { B7 Tunceli: Çemişgezek, } 2 \mathrm{~km} \text { from Vişneli } \\
\text { to Arpaderen }\end{array}$ & 1080 & 21.06 .2014 & \multirow{2}{*}{$\begin{array}{l}\text { Steppe, } \\
\text { field side, } \\
\text { ruderal, } \\
\text { degraded } \\
\text { oak forest }\end{array}$} & \multirow[b]{2}{*}{$*$} & \multirow[b]{2}{*}{ Ir.-Tur. } \\
\hline & 5460 & B7 Tunceli: Hozat, S of Kurukaymak & 1405 & 21.07.2014 & & & \\
\hline \multirow{4}{*}{ Crepis alpina $\mathrm{L}$. } & 3737 & B7 Tunceli: Center, $1 \mathrm{~km} \mathrm{~S}$ of Aktuluk & 930 & 22.05 .2014 & \multirow{4}{*}{ Steppe } & & \multirow{4}{*}{ Unk. } \\
\hline & 4067 & $\begin{array}{l}\text { B7 Tunceli: Ovacık, } 32 \mathrm{~km} \text { from Tunceli to } \\
\text { Ovacık (Munzur Valley) }\end{array}$ & 1110 & 26.05 .2014 & & & \\
\hline & 3935 & $\begin{array}{l}\text { B7 Tunceli: Center, } 2.5 \mathrm{~km} \text { from Tunceli to } \\
\text { Ovacık (Munzur Valley) }\end{array}$ & 930 & 25.05 .2014 & & & \\
\hline & 3814 & $\begin{array}{l}\text { B7 Tunceli: Center, } 3 \mathrm{~km} \text { from Tunceli- } \\
\text { Pülümür-Sütlüce crossroads to Sütlüce }\end{array}$ & 1070 & 23.05 .2014 & & & \\
\hline $\begin{array}{l}\text { Crepis commutata (Spreng.) } \\
\text { Greuter }\end{array}$ & 4496 & $\begin{array}{l}\text { B7 Tunceli: Pertek, between Çemişgezek and } \\
\text { Elazığ, } 6 \mathrm{~km} \text { from Bulgurtepe to Payamdüzü }\end{array}$ & 980 & 06.06 .2014 & Steppe & Red. & Unk. \\
\hline \multirow{2}{*}{$\begin{array}{l}\text { Crepis foetida } \text { L. subsp. } \\
\text { rhoeadifolia (M.Bieb.) Čelak. }\end{array}$} & 3880 & $\begin{array}{l}\text { B7 Tunceli: Pertek, } 5 \mathrm{~km} \text { from Pertek to } \\
\text { Çemişgezek }\end{array}$ & 866 & 24.05 .2014 & \multirow{2}{*}{$\begin{array}{l}\text { Field side, } \\
\text { steppe }\end{array}$} & & \multirow{2}{*}{ Unk. } \\
\hline & 5471 & $\begin{array}{l}\text { B8 Tunceli: Pülümür, between Derindere and } \\
\text { Bardakçı }\end{array}$ & 1840 & 22.07 .2014 & & & \\
\hline $\begin{array}{l}\text { Crepis sancta (L.) Bornm. subsp. } \\
\text { obovata (Boiss. \& Noë) Babc. }\end{array}$ & 3932 & $\begin{array}{l}\text { B7 Tunceli: Center, } 2.5 \mathrm{~km} \text { from Tunceli to } \\
\text { Ovacık (Munzur Valley) }\end{array}$ & 930 & 25.05 .2014 & Steppe & & Unk. \\
\hline \multirow{2}{*}{$\begin{array}{l}\text { Crupina crupinastrum (Moris) } \\
\text { Vis. }\end{array}$} & 4072b & $\begin{array}{l}\text { B7 Tunceli: Mazgirt, between Tunceli and } \\
\text { Kovancılar, around of Kuşcu }\end{array}$ & 867 & 02.06 .2014 & \multirow{2}{*}{$\begin{array}{l}\text { Field side, } \\
\text { steppe }\end{array}$} & & \multirow[t]{2}{*}{ Unk. } \\
\hline & $\mathbf{3 7 4 0}$ & B7 Tunceli: Center, $1 \mathrm{~km} \mathrm{~S}$ of Aktuluk & 930 & 22.05 .2014 & & & \\
\hline Crupina vulgaris Cass. & $4072 a$ & $\begin{array}{l}\text { B7 Tunceli: Mazgirt, between Tunceli and } \\
\text { Kovancilar, around of Kuşcu }\end{array}$ & 867 & 02.06 .2014 & $\begin{array}{l}\text { Field side, } \\
\text { steppe }\end{array}$ & & Unk. \\
\hline $\begin{array}{l}\text { Cyanus depressus (M.Bieb.) } \\
\text { Soják }\end{array}$ & 4069 & $\begin{array}{l}\text { B7 Tunceli: Mazgirt, between Tunceli and } \\
\text { Kovancilar, around of Kuşcu }\end{array}$ & 867 & 02.06 .2014 & $\begin{array}{l}\text { Field side, } \\
\text { steppe }\end{array}$ & & Unk. \\
\hline Cyanus lanigerus (DC.) Holub & 6556 & $\begin{array}{l}\text { B7 Tunceli: Ovacık, } 5 \mathrm{~km} \text { from Işıkvuran to } \\
\text { Ağaçpınar }\end{array}$ & 2140 & 01.06 .2015 & Steppe & $*$ & Ir.-Tur. \\
\hline Cyanus pichleri (Boiss.) Holub & 4463 & $\begin{array}{l}\text { B7 Tunceli: Hozat, } 3 \mathrm{~km} \text { from Dervişcemal } \\
\text { to Balkaynar }\end{array}$ & 1675 & 06.06 .2014 & Steppe & Rcd. & Unk. \\
\hline $\begin{array}{l}\text { Cyanus triumfetti (All.) Dostál ex } \\
\text { Á.Löve \& D.Löve }\end{array}$ & 6647 & $\begin{array}{l}\text { B7 Tunceli: Pülümür, } 11 \text { km NW of Ardıçlı } \\
\text { (Gersunut) v. (Munzur Mt.) }\end{array}$ & 2204 & 27.06 .2015 & Steppe & & Unk. \\
\hline $\begin{array}{l}\text { Cymbolaena griffithii (A.Gray) } \\
\text { Wagenitz }\end{array}$ & 3891 & $\begin{array}{l}\text { B7 Tunceli: Pertek, } 9 \mathrm{~km} \text { from Pertek to } \\
\text { Çemişgezek, } 2.5 \mathrm{~km} \text { before Singeç bridge }\end{array}$ & 865 & 24.05 .2014 & Steppe & & Ir.-Tur. \\
\hline \multirow[t]{2}{*}{ Cynara auranitica Post } & 5956 & $\begin{array}{l}\text { B7 Tunceli: Mazgirt, } 2 \mathrm{~km} \text { from } \\
\text { Beylermezrası to Göktepe }\end{array}$ & 870 & 09.08 .2014 & \multirow{2}{*}{$\begin{array}{l}\text { Field side, } \\
\text { steppe }\end{array}$} & \multirow[t]{2}{*}{ Red. } & \multirow[t]{2}{*}{ Ir.-Tur. } \\
\hline & 5719 & B7 Tunceli: Mazgirt, Kepektaşı & 860 & 25.07 .2014 & & & \\
\hline \multirow{2}{*}{$\begin{array}{l}\text { Erigeron acris L. subsp. } \\
\text { pycnotrichus (Vierh.) Grierson }\end{array}$} & 5443 & $\begin{array}{l}\text { B7 Tunceli: Hozat, N of Boydaş v. (Tahar } \\
\text { Valley) }\end{array}$ & 1215 & 21.07.2014 & \multirow{2}{*}{ Steppe } & & \multirow{2}{*}{$\begin{array}{l}\text { Euro.- } \\
\text { Sib. }\end{array}$} \\
\hline & 5373 & $\begin{array}{l}\text { B7 Tunceli: Nazımiye, } 2.5 \mathrm{~km} \text { E of Sarıyayla } \\
\text { v. }\end{array}$ & 2000 & 20.07 .2014 & & & \\
\hline $\begin{array}{l}\text { Erigeron caucasicus Steven. } \\
\text { subsp. venustus (Botsch.) } \\
\text { Grierson }\end{array}$ & 5808 & $\begin{array}{l}\text { B7 Tunceli: Ovacık, } 8 \mathrm{~km} \text { from Sarıül to } \\
\text { Yalmanlar, the walk way of Buyer Baba } \\
\text { sanctuary }\end{array}$ & 2680 & 06.08 .2014 & Steppe & & Unk. \\
\hline \multirow{3}{*}{ Eupatorium cannabinum L. } & 6255 & $\begin{array}{l}\text { B7 Tunceli: Nazımiye, Pülümür Valley, } 31 \\
\text { km from Tunceli to Pülümür }\end{array}$ & 1085 & 05.09 .2014 & \multirow{3}{*}{$\begin{array}{l}\text { Wet } \\
\text { places, } \\
\text { steppe }\end{array}$} & & \multirow{3}{*}{$\begin{array}{l}\text { Euro.- } \\
\text { Sib. }\end{array}$} \\
\hline & 5578 & $\begin{array}{l}\text { B7 Tunceli: Pülümür, } 5 \mathrm{~km} \text { from Erzincan- } \\
\text { Erzurum-Tunceli crossroads to Pülümür }\end{array}$ & 1420 & 22.07 .2014 & & & \\
\hline & 5991 & $\begin{array}{l}\text { B7 Tunceli: Pülümür, } 1.5 \mathrm{~km} \text { from Turnadere } \\
\text { to Közlüce }\end{array}$ & 1500 & 14.08 .2014 & & & \\
\hline Filago arvensis L. & 4498 & $\begin{array}{l}\text { B7 Tunceli: Pertek, between Çemişgezek and } \\
\text { Elazı̆̆ } 6 \mathrm{~km} \text { from Bulgurtepe to Payamdüzü }\end{array}$ & 980 & 06.06 .2014 & Steppe & & Unk. \\
\hline $\begin{array}{l}\text { Garhadiolus hedypnois Jaub. \& } \\
\text { Spach }\end{array}$ & 4424 & $\begin{array}{l}\text { B7 Tunceli: Pülümür, } 9 \mathrm{~km} \text { from Pülümür to } \\
\text { Tunceli (Pülümür valley), S of Kangallı v. }\end{array}$ & 1324 & 05.06 .2014 & Steppe & & Ir.-Tur. \\
\hline $\begin{array}{l}\text { Gundelia dersim Vitek, Yüce \& } \\
\text { Ergin }\end{array}$ & 3704 & $\begin{array}{l}\text { B7 Tunceli: Center, Tunceli - Elazığ road, } \\
700 \text { m after Aktuluk v. }\end{array}$ & 930 & 22.05 .2014 & Steppe & $*$ & Ir.-Tur. \\
\hline
\end{tabular}




\begin{tabular}{|c|c|c|c|c|c|c|c|}
\hline & 5086 & $\begin{array}{l}\text { B7 Tunceli: Çemişgezek, between } \\
\text { Çemişgezek and Elazı ğ, } 3 \mathrm{~km} \text { NW of } \\
\text { Sarıbalta v. }\end{array}$ & 1050 & 21.06 .2014 & & & \\
\hline & 3766 & $\begin{array}{l}\text { B7 Tunceli: Pertek, between Beydamı and } \\
\text { Yolkanak }\end{array}$ & 1300 & 22.05 .2014 & & & \\
\hline & 5065 & $\begin{array}{l}\text { B7 Tunceli: Pertek, } 17 \mathrm{~km} \text { from Pertek to } \\
\text { Tunceli }\end{array}$ & 1300 & 21.06.2014 & & & \\
\hline Gundelia glabra Mill. & 5797 & $\begin{array}{l}\text { B7 Tunceli: Ovacık, } 9 \mathrm{~km} \text { from Kocatepe to } \\
\text { Yalmanlar }\end{array}$ & 2510 & 06.08 .2014 & Steppe & * & Ir.-Tur. \\
\hline $\begin{array}{l}\text { Gundelia munzuriensis Vitek, } \\
\text { Yüce \& Ergin }\end{array}$ & Obs. & $\begin{array}{l}\text { B7 Tunceli: Ovacık, } 5 \text { km from Ovacık- } \\
\text { Tunceli-Yakatarla to Yakatarla }\end{array}$ & 1620 & 04.06 .2014 & Steppe & * & Ir.-Tur. \\
\hline Gundelia vitekii Armağan & Obs. & B7 Tunceli: Pülümür, Çakırkaya v. & 1750 & 05.06 .2014 & Steppe & $*$ & Ir.-Tur. \\
\hline Helianthus tuberosus L. & 6286 & $\begin{array}{l}\text { B7 Tunceli: Pülümür, } 4.5 \mathrm{~km} \text { from Pülümür } \\
\text { to Tunceli (Pülümür valley) }\end{array}$ & 1384 & 20.09 .2014 & In gardens & & Unk. \\
\hline $\begin{array}{l}\text { Helichrysum arenarium (L.) } \\
\text { Moench subsp. aucheri (Boiss.) } \\
\text { P.H.Davis \& Kupicha }\end{array}$ & 5609 & B7 Tunceli: Nazımiye, $7 \mathrm{~km}$ E of Ayranlı v. & 1910 & 23.07 .2014 & Steppe & $*$ & Ir.-Tur. \\
\hline $\begin{array}{l}\text { Helichrysum arenarium }(\mathrm{L} .) \\
\text { Moench subsp. rubicundum } \\
\text { (K.Koch) P.H.Davis \& Kupicha }\end{array}$ & 6061 & B7 Tunceli: Center, $1 \mathrm{~km} \mathrm{~W}$ of Babaocağ $1 \mathrm{v}$. & 1240 & 15.08 .2014 & Steppe & & Ir.-Tur. \\
\hline $\begin{array}{l}\text { Helichrysum plicatum DC. subsp. } \\
\text { plicatum }\end{array}$ & 6101 & $\begin{array}{l}\text { B7 Tunceli: Pülümür, } 6.5 \mathrm{~km} \text { from Pülümür } \\
\text { to Ardıçlı (Gersunut) v. }\end{array}$ & 1650 & 16.08 .2014 & Steppe & & Unk. \\
\hline Hieracium pannosum Boiss. & 5230 & $\begin{array}{l}\text { B7 Tunceli: Nazımiye, } 800 \mathrm{~m} \text { from } \\
\text { Așağırabat bridge to Büyükyurt (Hakis) v. }\end{array}$ & 1070 & 30.06 .2014 & $\begin{array}{l}\begin{array}{l}\text { Rocky } \\
\text { areas }\end{array} \\
\end{array}$ & & E.Medit. \\
\hline $\begin{array}{l}\text { Inula acaulis Schott \& Kotschy } \\
\text { ex Boiss. }\end{array}$ & 5802 & $\begin{array}{l}\text { B7 Tunceli: Ovacik, } 9.5 \mathrm{~km} \text { from Kocatepe to } \\
\text { Yalmanlar (the walk way of Buyer Baba } \\
\text { sanctuary) }\end{array}$ & 2600 & 06.08 .2014 & Steppe & & Unk. \\
\hline $\begin{array}{l}\text { Inula helenium L. subsp. } \\
\text { pseudohelenium Grierson }\end{array}$ & 5536 & B8 Tunceli: Pülümür, Şampaşakaraderbent v. & 1912 & 22.07 .2014 & $\begin{array}{l}\text { Wet } \\
\text { places, } \\
\text { degraded } \\
\text { oak forest }\end{array}$ & & Ir.-Tur. \\
\hline $\begin{array}{l}\text { Inula macrocephala Boiss. \& } \\
\text { Kotschy ex Boiss. }\end{array}$ & 6040 & $\begin{array}{l}\text { B7 Tunceli: Mazgirt, } 5.5 \mathrm{~km} \text { from Kale to } \\
\text { Doludizgin }\end{array}$ & 1560 & 15.08 .2014 & Steppe & $\begin{array}{l}* \\
\text { Rcd. }\end{array}$ & Ir.-Tur. \\
\hline Inula oculus-christi $\mathrm{L}$. & 5372 & $\begin{array}{l}\text { B7 Tunceli: Nazımiye, } 2.5 \text { km E of Sarıyayla } \\
\text { v. }\end{array}$ & 2000 & 20.07 .2014 & Steppe & & $\begin{array}{l}\text { Euro.- } \\
\text { Sib. }\end{array}$ \\
\hline $\begin{array}{l}\text { Inula thapsoides (M.Bieb. ex } \\
\text { Willd.) Spreng. }\end{array}$ & 5422 & $\begin{array}{l}\text { B7 Tunceli: Hozat, between Karaçavuş and } \\
\text { Boydaş }\end{array}$ & 1720 & 21.07 .2014 & Steppe & Red. & Unk. \\
\hline Inula viscidula Boiss. \& Kotschy & 6018 & B8 Tunceli: Pülümür, $2 \mathrm{~km} \mathrm{~W}$ of Ağaşenliği & 1740 & 14.08 .2014 & Steppe & Rcd. & Unk. \\
\hline \multirow{4}{*}{$\begin{array}{l}\text { Jurinea cataonica Boiss. \& } \\
\text { Hausskn. }\end{array}$} & 5593 & $\begin{array}{l}\text { B7 Tunceli: Nazımiye, between Ramazanköy } \\
\text { and Ayranlı }\end{array}$ & 1420 & 23.07 .2014 & \multirow{4}{*}{$\begin{array}{l}\text { Steppe } \\
\text { slopes }\end{array}$} & & \multirow{4}{*}{ Ir.-Tur. } \\
\hline & 5895 & $\begin{array}{l}\text { B7 Tunceli: Pülümür, } 42 \mathrm{~km} \text { from Tunceli to } \\
\text { Pülümür (Pülümür Valley), across of } \\
\text { Ağlayan Kayalar }\end{array}$ & 1570 & 07.08 .2014 & & & \\
\hline & 6188 & $\begin{array}{l}\text { B7 Tunceli: Center, } 17 \mathrm{~km} \text { from Tunceli- } \\
\text { Pülümür-Sütlüce crossroads to Çıralı v. ( } 5 \mathrm{~km} \\
\text { before Çıralı v.) }\end{array}$ & 1574 & 27.08 .2014 & & & \\
\hline & 6014 & $\begin{array}{l}\text { B8 Tunceli: Pülümür, } 2 \mathrm{~km} \text { from Balpayam } \\
\text { to Ağaşenliği }\end{array}$ & 1830 & 14.08.2014 & & & \\
\hline $\begin{array}{l}\text { Jurinea pontica Hausskn. \& } \\
\text { Freyn ex Hausskn. }\end{array}$ & 5177 & $\begin{array}{l}\text { B7 Tunceli: Çemişgezek, } 2.6 \mathrm{~km} \text { from } \\
\text { Çemişgezek to Gedikler }\end{array}$ & 1100 & 29.06 .2014 & Steppe & $\begin{array}{l}* \\
\text { Rcd. }\end{array}$ & Ir.-Tur. \\
\hline \multirow{2}{*}{$\begin{array}{l}\text { Jurinella moschus (Hablitz) } \\
\text { Bobrov }\end{array}$} & 4846 & $\begin{array}{l}\text { B8 Tunceli: Pülümür, } 500 \mathrm{~m} \mathrm{~N} \text { of Balpayam } \\
\text { (3-4 km S of Kırklar v.) }\end{array}$ & 2400 & 18.06 .2014 & \multirow[t]{2}{*}{ Mt. steppe } & \multirow[t]{2}{*}{ Rcd. } & \multirow[t]{2}{*}{ Ir.-Tur. } \\
\hline & 6678 & B8 Tunceli: Pülümür, 6 km E of Kırklar v. & 2310 & 09.06 .2015 & & & \\
\hline \multirow{2}{*}{$\begin{array}{l}\text { Klasea cerinthifolia }(\mathrm{Sm} .) \\
\text { Greuter \& Wagenitz }\end{array}$} & 5091 & B7 Tunceli: Çemişgezek, center & 946 & 21.06 .2014 & \multirow{2}{*}{ Steppe } & & \multirow{2}{*}{ Unk. } \\
\hline & 4530 & B7 Tunceli: Mazgirt, 1 km W of Kayacı v. & 990 & 16.06 .2014 & & & \\
\hline \multirow{2}{*}{$\begin{array}{l}\text { Klasea serratuloides (DC.) } \\
\text { Greuter \& Wagenitz }\end{array}$} & 4933 & $\begin{array}{l}\text { B7 Tunceli: Ovacık, } 12 \mathrm{~km} \text { from Işıkvuran to } \\
\text { Eskigedik }\end{array}$ & 1985 & 19.06.2014 & \multirow{2}{*}{ Steppe } & & \multirow{2}{*}{ Ir.-Tur. } \\
\hline & 4327 & $\begin{array}{l}\text { B7 Tunceli: Pülümür, } 6 \mathrm{~km} \text { from Kocatepe to } \\
\text { Çakırkaya }\end{array}$ & 1510 & 05.06 .2014 & & & \\
\hline \multirow{3}{*}{$\begin{array}{l}\text { Lactuca hispida } \mathrm{L} \text {. } \\
\text { (=Cephalorrhynchus tuberosus } \\
\text { (Steven) Schchian) }\end{array}$} & 4025 & $\begin{array}{l}\text { B7 Tunceli: Center, } 26 \mathrm{~km} \text { from Tunceli to } \\
\text { Ovacik (Munzur Valley) }\end{array}$ & 1060 & 26.05 .2014 & \multirow{3}{*}{$\begin{array}{l}\text { Degraded } \\
\text { oaks } \\
\text { forest, } \\
\text { steppe }\end{array}$} & & \multirow{3}{*}{ Unk. } \\
\hline & $\mathbf{5 8 8 7}$ & $\begin{array}{l}\text { B7 Tunceli: Nazımiye, } 8 \mathrm{~km} \mathrm{NE} \text { of } \\
\text { Büyükyurt (Hakis) v. }\end{array}$ & 1963 & 07.08 .2014 & & & \\
\hline & 4204 & $\begin{array}{l}\text { B7 Tunceli: Ovacık, } 1.5 \mathrm{~km} \text { from Ovacık- } \\
\text { Tunceli-Yakatarla crossroads to Yakatarla v. } \\
\end{array}$ & 1320 & 04.06 .2014 & & & \\
\hline $\begin{array}{l}\text { Lactuca mulgedioides (Vis. \& } \\
\text { Pančić) Boiss. \& Kotschy ex } \\
\text { Boiss. }\end{array}$ & 4712 & $\begin{array}{l}\text { B7 Tunceli: Hozat, between Hozat and } \\
\text { Ovacık, } 2 \mathrm{~km} \text { from Yüceldi to Aşlıca }\end{array}$ & 1970 & 17.06 .2014 & Steppe & & Ir.-Tur. \\
\hline Lactuca orientalis (Boiss.) Boiss. & 6053 & B7 Tunceli: Mazgirt, Yukarıyumca v. & 1430 & 15.08 .2014 & Steppe & & Ir.-Tur. \\
\hline \multirow{2}{*}{$\begin{array}{l}\text { Lapsana communis L. subsp. } \\
\text { intermedia (M.Bieb) Hayek var. } \\
\text { intermedia }\end{array}$} & 3689 & $\begin{array}{l}\text { B7 Tunceli: Center, Tunceli - Elazı̆g road, } \\
700 \text { m after Aktuluk v. }\end{array}$ & 930 & 22.05 .2014 & \multirow{2}{*}{$\begin{array}{l}\text { Degraded } \\
\text { oaks } \\
\text { forest, } \\
\text { steppe }\end{array}$} & & \multirow{2}{*}{ Unk. } \\
\hline & 5834 & $\begin{array}{l}\text { B7 Tunceli: Ovacık, } 800 \mathrm{~m} \mathrm{NE} \text { of Buyer } \\
\text { Baba Lake }\end{array}$ & 2910 & 06.08 .2014 & & & \\
\hline
\end{tabular}




\begin{tabular}{|c|c|c|c|c|c|c|c|}
\hline & 3967 & $\begin{array}{l}\text { B7 Tunceli: Center, } 2.5 \mathrm{~km} \text { from Tunceli- } \\
\text { Ovacık-Dedeağaç crossroads to Dedeağaç v. } \\
\text { (Munzur Valley) }\end{array}$ & 1100 & 25.05.2014 & & & \\
\hline & 3804 & $\begin{array}{l}\text { B7 Tunceli: Center, } 2 \mathrm{~km} \text { from Tunceli- } \\
\text { Pülümür-Sütlüce crossroads to Sütlüce }\end{array}$ & 1050 & 23.05.2014 & & & \\
\hline $\begin{array}{l}\text { Leontodon asperrimus (Willd.) } \\
\text { Endl. }\end{array}$ & 4175 & $\begin{array}{l}\text { B7 Tunceli: Nazımiye, } 5 \mathrm{~km} \text { from Nazımiye } \\
\text { to Tunceli }\end{array}$ & 1435 & 03.06 .2014 & Steppe & & Ir.-Tur. \\
\hline Onopordum acanthium L. & 5531 & B8 Tunceli: Pülümür, Şampaşakaraderbent v. & 1820 & 22.07 .2014 & Steppe & Rcd. & Unk. \\
\hline \multirow{3}{*}{ Onopordum candidum Nábělek } & 6125 & $\begin{array}{l}\text { B7 Tunceli: Çemişgezek, } 6 \mathrm{~km} \text { from } \\
\text { Çemişgezek to Pertek }\end{array}$ & 1108 & 17.08.2014 & \multirow{3}{*}{ Steppe } & & \multirow{3}{*}{ Ir.-Tur. } \\
\hline & 5717 & $\begin{array}{l}\text { B7 Tunceli: Mazgirt, } 4.5 \mathrm{~km} \text { from Kızılcık to } \\
\text { Kuşcu }\end{array}$ & 1000 & 25.07 .2014 & & & \\
\hline & 6273 & B7 Tunceli: Mazgirt, Kızılcık v. & 1014 & 19.09 .2014 & & & \\
\hline \multirow{2}{*}{$\begin{array}{l}\text { Onopordum carduchorum } \\
\text { Bornm. \& Beauverd }\end{array}$} & 5295 & $\begin{array}{l}\text { B7 Tunceli: Center, } 14 \mathrm{~km} \text { from Tunceli to } \\
\text { Pülümür (Pülümür Valley) }\end{array}$ & 970 & 20.07.2014 & \multirow[t]{2}{*}{ Steppe } & \multirow[t]{2}{*}{ Red. } & \multirow[t]{2}{*}{ Ir.-Tur. } \\
\hline & 5955 & B7 Tunceli: Mazgirt, NE of Aslanyurdu v. & 983 & 09.08 .2014 & & & \\
\hline \multirow{2}{*}{ Onopordum polycephalum Boiss. } & 5942 & $\begin{array}{l}\text { B7 Tunceli: Ovacık, } 3 \mathrm{~km} \text { from Ağaçpınar to } \\
\text { Işıkvuran }\end{array}$ & 1880 & 08.08.2014 & \multirow{2}{*}{ Steppe } & \multirow{2}{*}{$\begin{array}{l}* \\
\text { Rcd. }\end{array}$} & \multirow{2}{*}{ Ir.-Tur. } \\
\hline & 5943 & $\begin{array}{l}\text { B7 Tunceli: Ovacık, } 3 \mathrm{~km} \text { from Ağaçpınar to } \\
\text { Işıkvuran }\end{array}$ & 1880 & 08.08.2014 & & & \\
\hline Onopordum tauricum Willd. & 5143 & $\begin{array}{l}\text { B7 Tunceli: Çemişgezek, between Bozağaç } \\
\text { and Ulukale villages }\end{array}$ & 1100 & 28.06 .2014 & Steppe & Rcd. & $\begin{array}{l}\text { Euro.- } \\
\text { Sib. }\end{array}$ \\
\hline Onopordum turcicum Danin & 6082 & B8 Tunceli: Pülümür, SE of Kırklar v. & 1945 & 16.08 .2014 & Steppe & & Ir.-Tur. \\
\hline Picnomon acarna (L.) Cass. & 5722 & B7 Tunceli: Mazgirt, NE of Akdüven v. & 1040 & 05.08 .2014 & Steppe & & Unk. \\
\hline \multirow{2}{*}{ Picris hieracioides L. } & 6048 & $\begin{array}{l}\text { B7 Tunceli: Mazgirt, } 3 \mathrm{~km} \text { from Kale to } \\
\text { Doludizgin }\end{array}$ & 1710 & 15.08.2014 & \multirow{2}{*}{ Steppe } & \multirow{2}{*}{ Rcd. } & \multirow{2}{*}{$\begin{array}{l}\text { Euro.- } \\
\text { Sib. }\end{array}$} \\
\hline & 6081 & $\begin{array}{l}\text { B8 Tunceli: Pülümür, } 3 \mathrm{~km} \text { from Hasangazi } \\
\text { to Kurklar }\end{array}$ & 1890 & 16.08.2014 & & & \\
\hline $\begin{array}{l}\text { Pilosella echioides (Lumn.) } \\
\text { F.W.Schultz \& Sch.Bip. }\end{array}$ & 6049 & $\begin{array}{l}\text { B7 Tunceli: Mazgirt, } 2 \mathrm{~km} \text { from Kale to } \\
\text { Doludizgin }\end{array}$ & 1750 & 15.08 .2014 & Steppe & & Unk. \\
\hline $\begin{array}{l}\text { Pilosella verruculata (Link) } \\
\text { Soják }\end{array}$ & 5371 & $\begin{array}{l}\text { B7 Tunceli: Nazımiye, } 2.5 \mathrm{~km} \text { E of Sarıyayla } \\
\text { v. }\end{array}$ & 2000 & 20.07 .2014 & Steppe & Red. & Unk. \\
\hline \multirow{2}{*}{$\begin{array}{l}\text { Pilosella x auriculoides (Láng) } \\
\text { Arv. -Touv. }\end{array}$} & 5992 & $\begin{array}{l}\text { B7 Tunceli: Pülümür, } 1.5 \mathrm{~km} \text { from Turnadere } \\
\text { to Közlüce }\end{array}$ & 1500 & 14.08.2014 & \multirow{2}{*}{$\begin{array}{l}\text { Steppe, } \\
\text { pastures }\end{array}$} & & \multirow[b]{2}{*}{ Unk. } \\
\hline & 4058 & $\begin{array}{l}\text { B7 Tunceli: Ovacık, } 48 \mathrm{~km} \text { from Tunceli to } \\
\text { Ovacık, } 10 \mathrm{~km} \text { before Ovacık (Munzur } \\
\text { Valley) }\end{array}$ & 1190 & 26.05.2014 & & & \\
\hline $\begin{array}{l}\text { Psephellus goeksunensis (Aytaç } \\
\text { \& H.Duman) Greuter \& Raab- } \\
\text { Straube }\end{array}$ & 5010 & $\begin{array}{l}\text { B7 Tunceli: Ovacık, } 10 \mathrm{~km} \text { from Eğripınar to } \\
\text { Yenikonak }\end{array}$ & 1570 & 20.06 .2014 & Steppe & $\begin{array}{l}* \\
\text { Rcd. }\end{array}$ & Unk. \\
\hline \multirow{3}{*}{$\begin{array}{l}\text { Psephellus pyrrhoblepharus } \\
\text { (Boiss.) Wagenitz }\end{array}$} & 6474 & $\begin{array}{l}\text { B7 Tunceli: Nazımiye, Pülümür Valley, } 38 \\
\text { km from Tunceli to Pülümür }\end{array}$ & 1133 & 29.05.2015 & \multirow{3}{*}{$\begin{array}{l}\text { Mt. steppe, } \\
\text { rocky } \\
\text { areas }\end{array}$} & \multirow{3}{*}{$*$} & \multirow{3}{*}{ Ir.-Tur. } \\
\hline & 4276 & $\begin{array}{l}\text { B7 Tunceli: Ovacık, } 3 \text { km N of Işıkvuran v. } \\
\text { Munzur Mt. }\end{array}$ & 2120 & 04.06 .2014 & & & \\
\hline & 4338 & $\begin{array}{l}\text { B7 Tunceli: Pülümür, between Sarıgül and } \\
\text { Kocatepe }\end{array}$ & 1905 & 05.06.2014 & & & \\
\hline $\begin{array}{l}\text { Ptilostemon afer (Jacq.) Greuter } \\
\text { subsp. eburneus Greuter }\end{array}$ & 5317 & $\begin{array}{l}\text { B7 Tunceli: Nazımiye, } 2 \mathrm{~km} \text { from Nazımiye } \\
\text { to Tunceli }\end{array}$ & 1548 & 20.07.2014 & Steppe & $*$ & Ir.-Tur. \\
\hline Pulicaria dysenterica (L.) Bernh. & 6136 & B7 Tunceli: Mazgirt, S of Dedebağ v. & 860 & 26.08 .2014 & Wet places & Rcd. & Unk. \\
\hline Pulicaria vulgaris (L.) Gaertn. & 6050 & B7 Tunceli: Mazgirt, around of Kale v. & 1590 & 15.08 .2014 & Steppe & Rcd. & $\begin{array}{l}\text { Euro.- } \\
\text { Sib. }\end{array}$ \\
\hline $\begin{array}{l}\text { Reichardia dichotoma (Vahl) } \\
\text { Freyn }\end{array}$ & 5784 & $\begin{array}{l}\text { B7 Tunceli: Pülümür, between Kocatepe and } \\
\text { Çakırkaya }\end{array}$ & 1710 & 06.08 .2014 & Steppe & & Ir.-Tur. \\
\hline \multirow[b]{2}{*}{ Rhagadiolus stellatus (L.) Gaertn. } & 3728 & B7 Tunceli: Center, $1 \mathrm{~km} \mathrm{~S}$ of Aktuluk & 930 & 22.05 .2014 & \multirow[b]{2}{*}{ Steppe } & \multirow[b]{2}{*}{ Red. } & \\
\hline & 4174 & $\begin{array}{l}\text { B7 Tunceli: Nazımiye, } 5 \mathrm{~km} \text { from Nazımiye } \\
\text { to Tunceli }\end{array}$ & 1435 & 03.06.2014 & & & Medit. \\
\hline & 6546 & $\begin{array}{l}\text { B7 Tunceli: Ovacık, } 9 \mathrm{~km} \text { from } \\
\text { Aşağıtorunoba to Çakmaklı }\end{array}$ & 1800 & 01.06 .2015 & & & \\
\hline Scorzonera incisa DC. & 6545 & $\begin{array}{l}\text { B7 Tunceli: Ovacık, } 9 \mathrm{~km} \text { from } \\
\text { Aşağıtorunoba to Çakmaklı }\end{array}$ & 1800 & 01.06 .2015 & Steppe & & Ir.-Tur. \\
\hline & 4417 & $\begin{array}{l}\text { B7 Tunceli: Pülümür, between Kabadal and } \\
\text { Sağlamtaş }\end{array}$ & 1540 & 05.06 .2014 & & & \\
\hline & 5910 & B7 Tunceli: Ovacik, SW of Yakatarla v. & 1700 & 08.08 .2014 & & & \\
\hline $\begin{array}{l}\text { Scorzonera latifolla (Fisch. \& } \\
\text { C.A.Mey.) DC. }\end{array}$ & 5472 & $\begin{array}{l}\text { B8 Tunceli: Pülümür, between Derindere and } \\
\text { Bardakçı }\end{array}$ & 1840 & 22.07.2014 & Steppe & & Ir.-Tur. \\
\hline & 4152 & $\begin{array}{l}\text { B7 Tunceli: Mazgirt, } 2 \mathrm{~km} \text { from } \\
\text { Beylermezrası to Aslanyurdu }\end{array}$ & 855 & 02.06.2014 & & & \\
\hline Scorzonera papposa DC. & 3749 & $\begin{array}{l}\text { B7 Tunceli: Center, } 1 \mathrm{~km} \text { from Kopuzlar to } \\
\text { Yolkonak }\end{array}$ & 1114 & 22.05 .2014 & Steppe & & Ir.-Tur. \\
\hline & 4103 & $\begin{array}{l}\text { B7 Tunceli: Mazgirt, } 7 \mathrm{~km} \text { from Kizılcık to } \\
\text { Göktepe }\end{array}$ & 935 & 02.06 .2014 & & & \\
\hline & 5167 & $\begin{array}{l}\text { B7 Tunceli: Çemişgezek, Tahar valley (Ali } \\
\text { Boğazı) }\end{array}$ & 1024 & 29.06.2014 & & $*$ & \\
\hline 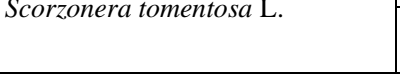 & 5908 & $\begin{array}{l}\text { B7 Tunceli: Ovacık, } 9 \text { km from Tunceli- } \\
\text { Ovacık-Yakatarla crossroads to Yakatarla }\end{array}$ & 1620 & 08.08.2014 & S & $*$ & \\
\hline
\end{tabular}




\begin{tabular}{|c|c|c|c|c|c|c|c|}
\hline Scorzonera veratrifolia Fenzl & 6021 & $\begin{array}{l}\text { B8 Tunceli: Pülümür, S of Ağaşenliği v., } \\
\text { between Ağaşenliği and Altınhüseyin }\end{array}$ & 1650 & 14.08 .2014 & Steppe & & Ir.-Tur. \\
\hline \multirow{2}{*}{ Senecio cilicius Boiss. } & 5877 & $\begin{array}{l}\text { B7 Tunceli: Nazımiye, } 6 \mathrm{~km} \mathrm{NE} \text { of } \\
\text { Büyükyurt (Hakis) v. }\end{array}$ & 2015 & 07.08 .2014 & \multirow{2}{*}{ Steppe } & \multirow[b]{2}{*}{$*$} & \multirow{2}{*}{ Ir.-Tur. } \\
\hline & 5377 & $\begin{array}{l}\text { B7 Tunceli: Nazımiye, } 2.5 \mathrm{~km} \text { E of Sarıyayla } \\
\text { v. }\end{array}$ & 2000 & 20.07 .2014 & & & \\
\hline \multirow[b]{2}{*}{ Senecio othonnae M.Bieb. } & 5375 & $\begin{array}{l}\text { B7 Tunceli: Nazımiye, } 2.5 \mathrm{~km} \text { E of Sarıyayla } \\
\text { v. }\end{array}$ & 2000 & 20.07 .2014 & \multirow{2}{*}{$\begin{array}{l}\text { Wet } \\
\text { places, } \\
\text { steppe }\end{array}$} & & \multirow{2}{*}{$\begin{array}{l}\text { Euro.- } \\
\text { Sib. }\end{array}$} \\
\hline & 5772 & $\begin{array}{l}\text { B7 Tunceli: Pülümür, } 16 \mathrm{~km} \text { from Tunceli to } \\
\text { Pülümür (Pülümür Valley) }\end{array}$ & 1240 & 06.08 .2014 & & & \\
\hline $\begin{array}{l}\text { Senecio racemosus (M.Bieb.) } \\
\text { DC. }\end{array}$ & 6080 & $\begin{array}{l}\text { B8 Tunceli: Pülümür, } 3 \text { km from Hasangazi } \\
\text { to Kırklar }\end{array}$ & 1890 & 16.08 .2014 & Steppe & & Ir.-Tur. \\
\hline Senecio vernalis Waldst. \& Kit. & Obs. & $\begin{array}{l}\text { B7 Tunceli: Center, } 500 \mathrm{~m} \text { from Tunceli- } \\
\text { Pülümür-Sütlüce crossroads to Sütlüce v. }\end{array}$ & 940 & 23.05 .2014 & Steppe & & Unk. \\
\hline \multirow{3}{*}{ Siebera pungens (Lam.) J. Gay } & 4557 & $\begin{array}{l}\text { B7 Tunceli: Mazgirt, } 1 \mathrm{~km} \text { from Ortaharman } \\
\text { to Sülüntaş }\end{array}$ & 1220 & 16.06 .2014 & \multirow{3}{*}{ Steppe } & & \multirow{3}{*}{ Ir.-Tur. } \\
\hline & 5726 & B7 Tunceli: Mazgirt, $N$ of Akdüven v. & 1150 & 05.08 .2014 & & & \\
\hline & 5705 & $\begin{array}{l}\text { B7 Tunceli: Pertek, } 3 \mathrm{~km} \text { from Pınarlar to } \\
\text { Biçmekaya }\end{array}$ & 1310 & 25.07 .2014 & & & \\
\hline $\begin{array}{l}\text { Tanacetum argenteum (Lam.) } \\
\text { Willd. subsp. canum (K.Koch) } \\
\text { Grierson }\end{array}$ & 4012 & $\begin{array}{l}\text { B7 Tunceli: Center, } 17 \mathrm{~km} \text { from Tunceli to } \\
\text { Ovacık (Munzur Valley) }\end{array}$ & 1015 & 25.05 .2014 & On rocks & & Unk. \\
\hline \multirow{2}{*}{$\begin{array}{l}\text { Tanacetum armenum (DC.) } \\
\text { Sch.Bip. }\end{array}$} & 4271 & $\begin{array}{l}\text { B7 Tunceli: Ovacık, } 3 \text { km N of Işıkvuran v. } \\
\text { Munzur Mt. }\end{array}$ & 2100 & 04.06 .2014 & \multirow{2}{*}{$\begin{array}{l}\text { Rock } \\
\text { crevices }\end{array}$} & & \multirow{2}{*}{ Unk. } \\
\hline & 6535 & $\begin{array}{l}\text { B7 Tunceli: Ovacık, } 7 \mathrm{~km} \text { from Yoncalı to } \\
\text { Ağaçpınar }\end{array}$ & 2165 & 01.06 .2015 & & & \\
\hline \multirow{2}{*}{ Tanacetum balsamita $\mathrm{L}$. } & 5437 & $\begin{array}{l}\text { B7 Tunceli: Hozat, between Boydaş and } \\
\text { Karaçavuş }\end{array}$ & 1635 & 21.07.2014 & \multirow{2}{*}{ Steppe } & & \multirow{2}{*}{ Unk. } \\
\hline & 5376 & $\begin{array}{l}\text { B7 Tunceli: Nazımiye, } 2.5 \mathrm{~km} \text { E of Sarıyayla } \\
\text { v. }\end{array}$ & 2000 & 20.07 .2014 & & & \\
\hline $\begin{array}{l}\text { Tanacetum balsamitoides } \\
\text { Sch.Bip. }\end{array}$ & 5835 & $\begin{array}{l}\text { B7 Tunceli: Ovacık, around of Buyer Baba } \\
\text { Lake }\end{array}$ & 2940 & 06.08 .2014 & Riverside & & Unk. \\
\hline $\begin{array}{l}\text { Tanacetum cappadocicum (DC.) } \\
\text { Sch.Bip. }\end{array}$ & 4678 & $\begin{array}{l}\text { B7 Tunceli: Hozat, between Karacaköy and } \\
\text { Uzundal, around of Sarisaltuk Türbesi }\end{array}$ & 2270 & 17.06 .2014 & Steppe & $*$ & Ir.-Tur. \\
\hline $\begin{array}{l}\text { Tanacetum cilicium (Boiss.) } \\
\text { Grierson }\end{array}$ & 4436 & $\begin{array}{l}\text { B7 Tunceli: Center, } 16 \mathrm{~km} \text { from Aktuluk to } \\
\text { Demirkap1 }\end{array}$ & 1395 & 06.06 .2014 & Steppe & & E.Medit. \\
\hline \multirow{4}{*}{$\begin{array}{l}\text { Tanacetum mисroniferum Hub.- } \\
\text { Mor. \& Grierson }\end{array}$} & 5623 & B7 Tunceli: Nazımiye, $1 \mathrm{~km} \mathrm{~S}$ of Holik v. & 1990 & 23.07 .2014 & \multirow{4}{*}{$\begin{array}{l}\text { Rocky } \\
\text { areas, } \\
\text { steppe }\end{array}$} & \multirow{4}{*}{$*$} & \multirow{4}{*}{ Ir.-Tur. } \\
\hline & 4316 & B7 Tunceli: Ovacık, 1.5 km E of Ișıkvuran v. & 1960 & 04.06 .2014 & & & \\
\hline & 4237 & $\begin{array}{l}\text { B7 Tunceli: Ovacık, } 18 \mathrm{~km} \text { from Ovacık- } \\
\text { Tunceli-Işıkvuran crossroads to Işıkvuran }\end{array}$ & 1635 & 04.06 .2014 & & & \\
\hline & 4339 & $\begin{array}{l}\text { B7 Tunceli: Pülümür, between Sarıgül and } \\
\text { Kocatepe }\end{array}$ & 1900 & 05.06 .2014 & & & \\
\hline $\begin{array}{l}\text { Tanacetum nitens (Boiss. \& Noë) } \\
\text { Grierson }\end{array}$ & 4448 & $\begin{array}{l}\text { B7 Tunceli: Hozat, between Demirkapı and } \\
\text { Dervișcemal, } 300 \mathrm{~m} \text { to Buzlupınar junction }\end{array}$ & 1810 & 06.06 .2014 & \begin{tabular}{|l|} 
Rocky \\
areas
\end{tabular} & \begin{tabular}{|l|}
$*$ \\
Rcd.
\end{tabular} & Ir.-Tur. \\
\hline $\begin{array}{l}\text { Tanacetum polycephalum } \\
\text { Sch.Bip. subsp. argyrophyllum } \\
\text { (K.Koch) Podlech (=Tanacetum } \\
\text { argyrophyllum (K.Koch) } \\
\text { Tzvelev) }\end{array}$ & 4471 & $\begin{array}{l}\text { B7 Tunceli: Hozat, } 3.5 \mathrm{~km} \text { from Hozat to } \\
\text { Pertek, junction of Kardelen v. }\end{array}$ & 1470 & 06.06 .2014 & On rocks & Rcd. & Ir.-Tur. \\
\hline $\begin{array}{l}\text { Tanacetum zahlbruckneri (Náb.) } \\
\text { Grierson }\end{array}$ & 4387 & $\begin{array}{l}\text { B7 Tunceli: Pülümür, } 1 \mathrm{~km} \text { from Çakırkaya } \\
\text { and Dereboyu }\end{array}$ & 1800 & 05.06 .2014 & Steppe & $*$ & Ir.-Tur. \\
\hline \multirow{2}{*}{$\begin{array}{l}\text { Taraxacum montanum } \\
\text { (C.A.Mey.) DC. }\end{array}$} & 5841 & $\begin{array}{l}\text { B7 Tunceli: Nazımiye, } 2.5 \mathrm{~km} \mathrm{NE} \text { of } \\
\text { Büyükyurt (Hakis) v. }\end{array}$ & 1700 & 07.08 .2014 & \multirow{2}{*}{ Steppe } & \multirow{2}{*}{ Rcd. } & \multirow{2}{*}{ Ir.-Tur. } \\
\hline & 5753 & $\begin{array}{l}\text { B7 Tunceli: Nazımiye, Pülümür Valley, } 38 \\
\text { km from Tunceli to Pülümür }\end{array}$ & 1150 & 06.08 .2014 & & & \\
\hline $\begin{array}{l}\text { Taraxacum stevenii DC. } \\
\text { (=Taraxacum crepidiforme DC.) }\end{array}$ & 5798 & $\begin{array}{l}\text { B7 Tunceli: Ovacık, } 9 \mathrm{~km} \text { from Kocatepe to } \\
\text { Yalmanlar }\end{array}$ & 2510 & 06.08 .2014 & Steppe & & Ir.-Tur. \\
\hline $\begin{array}{l}\text { Tragopogon buphthalmoides } \\
\text { (DC.) Boiss. }\end{array}$ & 6073 & $\begin{array}{l}\text { B8 Tunceli: Pülümür, } 3 \text { km from Hasangazi } \\
\text { to Kırklar }\end{array}$ & 1890 & 16.08 .2014 & Steppe & & Ir.-Tur. \\
\hline $\begin{array}{l}\text { Tragopogon porrifolius } \text { L. subsp. } \\
\text { longirostris (Sch.Bip.) Greuter }\end{array}$ & 4209 & $\begin{array}{l}\text { B7 Tunceli: Ovacık, } 1.5 \mathrm{~km} \text { from Ovacık- } \\
\text { Tunceli-Yakatarla crossroads to Yakatarla v. }\end{array}$ & 1320 & 04.06 .2014 & Steppe & & Unk. \\
\hline $\begin{array}{l}\text { Tragopogon reticulatus Boiss. \& } \\
\text { Huet }\end{array}$ & 5799 & $\begin{array}{l}\text { B7 Tunceli: Ovacık, } 9 \mathrm{~km} \text { from Kocatepe to } \\
\text { Yalmanlar }\end{array}$ & 2510 & 06.08 .2014 & Steppe & & Unk. \\
\hline $\begin{array}{l}\text { Tripleurospermum monticolum } \\
\text { (Boiss. \& A.Huet) Bornm. }\end{array}$ & 6519 & $\begin{array}{l}\text { B7 Tunceli: Center, } 17 \mathrm{~km} \text { from Tunceli- } \\
\text { Pülümür-Sütlüce crossroads to Çıralı v. ( } 8 \mathrm{~km} \\
\text { before Çıralı v.) }\end{array}$ & 1810 & 01.06 .2015 & Steppe & Red. & Unk. \\
\hline Tussilago farfara $\mathrm{L}$. & Obs. & $\begin{array}{l}\text { B7 Tunceli: Center, } 14 \mathrm{~km} \text { from Tunceli to } \\
\text { Ovacık (Munzur Valley) }\end{array}$ & 1000 & 11.04 .2015 & Steppe & & $\begin{array}{l}\text { Euro.- } \\
\text { Sib. }\end{array}$ \\
\hline \multirow[t]{2}{*}{$\begin{array}{l}\text { Turanecio eriospermus (DC.) } \\
\text { Hamzaoğlu }\end{array}$} & 5812 & $\begin{array}{l}\text { B7 Tunceli: Ovacik, } 8 \mathrm{~km} \text { from Sarigül to } \\
\text { Yalmanlar, the walk way of Buyer Baba } \\
\text { sanctuary }\end{array}$ & 2680 & 06.08 .2014 & \multirow{2}{*}{$\begin{array}{l}\text { Rocky } \\
\text { areas, } \\
\text { steppe }\end{array}$} & & \multirow[t]{2}{*}{ Ir.-Tur. } \\
\hline & 4292 & B7 Tunceli: Ovacık, Ișıkvuran v. & 1710 & 04.06 .2014 & & & \\
\hline Xanthium spinosum L. & Obs. & B7 Tunceli: Çemişgezek, Bağsuyu v. & 980 & 21.06 .2014 & Steppe & & Unk. \\
\hline
\end{tabular}




\begin{tabular}{|c|c|c|c|c|c|c|c|}
\hline Xanthium strumarium L. & 6129 & B7 Tunceli: Mazgirt, S of Dedebağ v. & 860 & 26.08.2014 & $\begin{array}{l}\text { Wet } \\
\text { places, } \\
\text { steppe }\end{array}$ & & Unk. \\
\hline \multirow[t]{2}{*}{ Zoegea leptaurea L. } & 4504 & $\begin{array}{l}\text { B7 Tunceli: Çemişgezek, } 5 \mathrm{~km} \text { from } \\
\text { Payamdüzü to Yemişdere }\end{array}$ & 865 & 06.06 .2014 & \multirow[t]{2}{*}{ Steppe } & & \multirow[t]{2}{*}{ Ir.-Tur. } \\
\hline & 6134 & B7 Tunceli: Mazgirt, S of Dedebağ v. & 860 & 26.08 .2014 & & & \\
\hline \multicolumn{8}{|l|}{ BERBERIDACEAE Juss. } \\
\hline Berberis crataegina DC. & 5771 & $\begin{array}{l}\text { B7 Tunceli: Pülümür, } 16 \mathrm{~km} \text { from Tunceli to } \\
\text { Pülümür (Pülümür Valley) }\end{array}$ & 1240 & 06.08 .2014 & Steppe & & Ir.-Tur. \\
\hline $\begin{array}{l}\text { Bongardia chrysogenum (L.) } \\
\text { Spach }\end{array}$ & 6428 & $\begin{array}{l}\text { B7 Tunceli: Center, } 7 \mathrm{~km} \text { from Tunceli to } \\
\text { Pülümür (Pülümür Valley) }\end{array}$ & 934 & 12.04.2015 & Steppe & Rcd. & Ir.-Tur. \\
\hline Leontice leontopetalum $\mathrm{L}$. & 6429 & $\begin{array}{l}\text { B7 Tunceli: Center, } 7 \mathrm{~km} \text { from Tunceli to } \\
\text { Pülümür (Pülümür Valley) }\end{array}$ & 934 & 12.04.2015 & Steppe & & Unk. \\
\hline \multicolumn{8}{|l|}{ BETULACEAE Gray } \\
\hline $\begin{array}{l}\text { Alnus glutinosa (L.) Gaertn. } \\
\text { subsp. glutinosa }\end{array}$ & 5672 & $\begin{array}{l}\text { B7 Tunceli: Ovacık, } 16 \mathrm{~km} \text { from Ovacık to } \\
\text { Tunceli (Munzur Valley) }\end{array}$ & 1180 & 24.07 .2014 & Riverside & & $\begin{array}{l}\text { Euro.- } \\
\text { Sib. }\end{array}$ \\
\hline $\begin{array}{l}\text { Alnus glutinosa (L.) Gaertn. } \\
\text { subsp. antitaurica Yalt. }\end{array}$ & 6065 & $\begin{array}{l}\text { B7 Tunceli: Center, } 4 \mathrm{~km} \text { from Tunceli center } \\
\text { to Babaocağ } 1\end{array}$ & 930 & 15.08 .2014 & Riverside & & E.Medit. \\
\hline Corylus avellana $\mathrm{L}$. & 5572 & B8 Tunceli: Pülümür, 2 km N of Elmalı & 1400 & 22.07 .2014 & Riverside & & $\begin{array}{l}\text { Euro.- } \\
\text { Sib. }\end{array}$ \\
\hline \multicolumn{8}{|l|}{ BORAGINACEAE Juss. } \\
\hline Alkanna froedinii Rech.f. & 4360 & $\begin{array}{l}\text { B7 Tunceli: Pülümür, } 2 \mathrm{~km} \text { from Sarıgül to } \\
\text { Yalmanlar }\end{array}$ & 2200 & 05.06 .2014 & Steppe & $*$ & Ir.-Tur. \\
\hline Alkanna orientalis (L.) Boiss. & 5975 & $\begin{array}{l}\text { B7 Tunceli: Hozat, } 3.2 \mathrm{~km} \text { from Hozat to } \\
\text { Pertek }\end{array}$ & 1440 & 13.08.2014 & Steppe & & Ir.-Tur. \\
\hline $\begin{array}{l}\text { Anchusa leptophylla Roem. \& } \\
\text { Schhult. subsp. tomentosa } \\
\text { (Boiss.) D.F.Chamb. }\end{array}$ & 5272 & $\begin{array}{l}\text { B7 Tunceli: Pertek, } 3 \text { km from Pertek to } \\
\text { Tunceli }\end{array}$ & 1257 & 19.07.2014 & Steppe & $*$ & Ir.-Tur. \\
\hline Asperugo procumbens $\mathrm{L}$. & 6445 & B7 Tunceli: Center, Alibaba nh. & 930 & 11.04 .2015 & Steppe & Red. & $\begin{array}{l}\text { Euro.- } \\
\text { Sib. }\end{array}$ \\
\hline \multirow{4}{*}{ Brunnera orientalis I.M.Johnst. } & 6521 & $\begin{array}{l}\text { B7 Tunceli: Nazımiye, } 4 \mathrm{~km} \mathrm{NE} \text { of } \\
\text { Büyükyurt (Hakis) v. }\end{array}$ & 1707 & 01.06 .2015 & \multirow{4}{*}{$\begin{array}{l}\text { Degraded } \\
\text { oak forest, } \\
\text { steppe }\end{array}$} & & \multirow{4}{*}{ Euxine } \\
\hline & 6472 & $\begin{array}{l}\text { B7 Tunceli: Nazımiye, Pülümür Valley, } 38 \\
\text { km from Tunceli to Pülümür }\end{array}$ & 1127 & 29.05.2015 & & & \\
\hline & 6539 & $\begin{array}{l}\text { B7 Tunceli: Ovacık, } 7 \mathrm{~km} \text { from Yoncalı to } \\
\text { Ağaçpınar }\end{array}$ & 2015 & 01.06 .2015 & & & \\
\hline & 6400 & $\begin{array}{l}\text { B7 Tunceli: Center, } 12 \mathrm{~km} \text { from Tunceli to } \\
\text { Ovacık (Munzur Valley) }\end{array}$ & 1000 & 11.04 .2015 & & & \\
\hline Cerinthe minor $\mathrm{L}$. & 3992 & $\begin{array}{l}\text { B7 Tunceli: Center, between Tunceli and } \\
\text { Ovacık (Munzur Valley), road of Dilek v. }\end{array}$ & 1043 & 25.05 .2014 & Steppe & & $\begin{array}{l}\text { Euro.- } \\
\text { Sib. }\end{array}$ \\
\hline \multirow{3}{*}{ Cynoglossum creticum Mill. } & 6650 & $\begin{array}{l}\text { B7 Tunceli: Pülümür, } 5 \text { km NW of Ardıçlı } \\
\text { (Gersunut) v. }\end{array}$ & 2285 & 27.06.2015 & \multirow{3}{*}{ Steppe } & & \multirow{3}{*}{ Unk. } \\
\hline & 6481 & $\begin{array}{l}\text { B7 Tunceli: Pülümür, } 48 \mathrm{~km} \text { from Tunceli to } \\
\text { Pülümür (Pülümür Valley) }\end{array}$ & 1205 & 29.05 .2015 & & & \\
\hline & 3783 & $\begin{array}{l}\text { B7 Tunceli: Center, } 500 \mathrm{~m} \text { from Tunceli- } \\
\text { Pülümür-Sütlüce crossroads to Sütlüce v. }\end{array}$ & 940 & 23.05.2014 & & & \\
\hline \multirow{3}{*}{ Cynoglossum vanense Storý } & 4673 & $\begin{array}{l}\text { B7 Tunceli: Hozat, between Karacaköy and } \\
\text { Uzundal, around of Sarisaltuk Türbesi }\end{array}$ & 2270 & 17.06 .2014 & \multirow{3}{*}{ Steppe } & \multirow{3}{*}{ Red. } & \multirow{3}{*}{ Ir.-Tur. } \\
\hline & 4250 & Ir.-Tur. & 2020 & 04.06 .2014 & & & \\
\hline & 4778 & $\begin{array}{l}\text { B7 Tunceli: Pülümür, } 2 \mathrm{~km} \text { from Turnadere } \\
\text { to Közlüce }\end{array}$ & 1560 & 18.06 .2014 & & & \\
\hline Echium italicum $\mathrm{L}$. & 6010 & $\begin{array}{l}\text { B8 Tunceli: Pülümür, } 4 \text { km from Hasangazi } \\
\text { to Balpayam }\end{array}$ & 1832 & 14.08 .2014 & Steppe & & Medit. \\
\hline \multirow{2}{*}{$\begin{array}{l}\text { Macrotomia densiflora (Ledeb.) } \\
\text { McBride }\end{array}$} & 4299 & $\begin{array}{l}\text { B7 Tunceli: Ovacık, } 7 \mathrm{~km} \text { from Işıkvuran to } \\
\text { Ağaçpınar }\end{array}$ & 2123 & 04.06.2014 & \multirow{2}{*}{ Mt. steppe } & & \multirow{2}{*}{ Ir.-Tur. } \\
\hline & 4278 & $\begin{array}{l}\text { B7 Tunceli: Ovacık, } 3 \text { km N of Işıkvuran v. } \\
\text { Munzur Mt. }\end{array}$ & 2150 & 04.06 .2014 & & & \\
\hline $\begin{array}{l}\text { Myosotis sylvatica Hoffm. subsp. } \\
\text { rivularis Vestergr. }\end{array}$ & 5953 & B7 Tunceli: Ovacık, W of Ziyaret v. & 1335 & 08.08.2014 & Riverside & & Unk. \\
\hline Nonea persica Boiss. & 4036 & $\begin{array}{l}\text { B7 Tunceli: Ovacık, } 41 \mathrm{~km} \text { from Tunceli to } \\
\text { Ovacık (Munzur Valley) }\end{array}$ & 1175 & 26.05 .2014 & Steppe & Red. & Ir.-Tur. \\
\hline $\begin{array}{l}\text { Onosma alborosea Fisch. \& } \\
\text { C.A.Mey. subsp. sanguinolenta } \\
\text { (Vatke) Bornm. }\end{array}$ & 4680 & $\begin{array}{l}\text { B7 Tunceli: Hozat, between Karacaköy and } \\
\text { Uzundal, around of Sarısaltuk Türbesi }\end{array}$ & 2270 & 17.06 .2014 & Steppe & Red. & Ir.-Tur. \\
\hline Onosma malatyana Binzet & 7516 & $\begin{array}{l}\text { B7 Tunceli: Mazgirt, } 1.5 \mathrm{~km} \text { from Kızılcık to } \\
\text { Göktepe }\end{array}$ & 965 & 03.06.2017 & Steppe & & Ir.-Tur. \\
\hline Onosma sericea Willd. & Obs. & $\begin{array}{l}\text { B7 Tunceli: Center, Tunceli - Elazığ road, } \\
700 \text { m after Aktuluk v. }\end{array}$ & 930 & 22.05 .2014 & Steppe & & Ir.-Tur. \\
\hline \multirow{3}{*}{$\begin{array}{l}\text { Paracaryum cristatum (Schreb.) } \\
\text { Boiss. }\end{array}$} & 4001 & $\begin{array}{l}\text { B7 Tunceli: Center, between Tunceli and } \\
\text { Ovacık (Munzur Valley), road of Dilek v. }\end{array}$ & 995 & 25.05 .2014 & \multirow{3}{*}{$\begin{array}{l}\text { Steppe, } \\
\text { rocky } \\
\text { areas }\end{array}$} & \multirow{3}{*}{$*$} & \\
\hline & 4570 & B7 Tunceli: Mazgirt, 1 km S of Geçitveren v. & 1345 & 16.06 .2014 & & & \\
\hline & 5026 & $\begin{array}{l}\text { B7 Tunceli: Ovacık, } 10 \mathrm{~km} \text { from Eğripınar to } \\
\text { Yenikonak }\end{array}$ & 1570 & 20.06 .2014 & & & \\
\hline
\end{tabular}




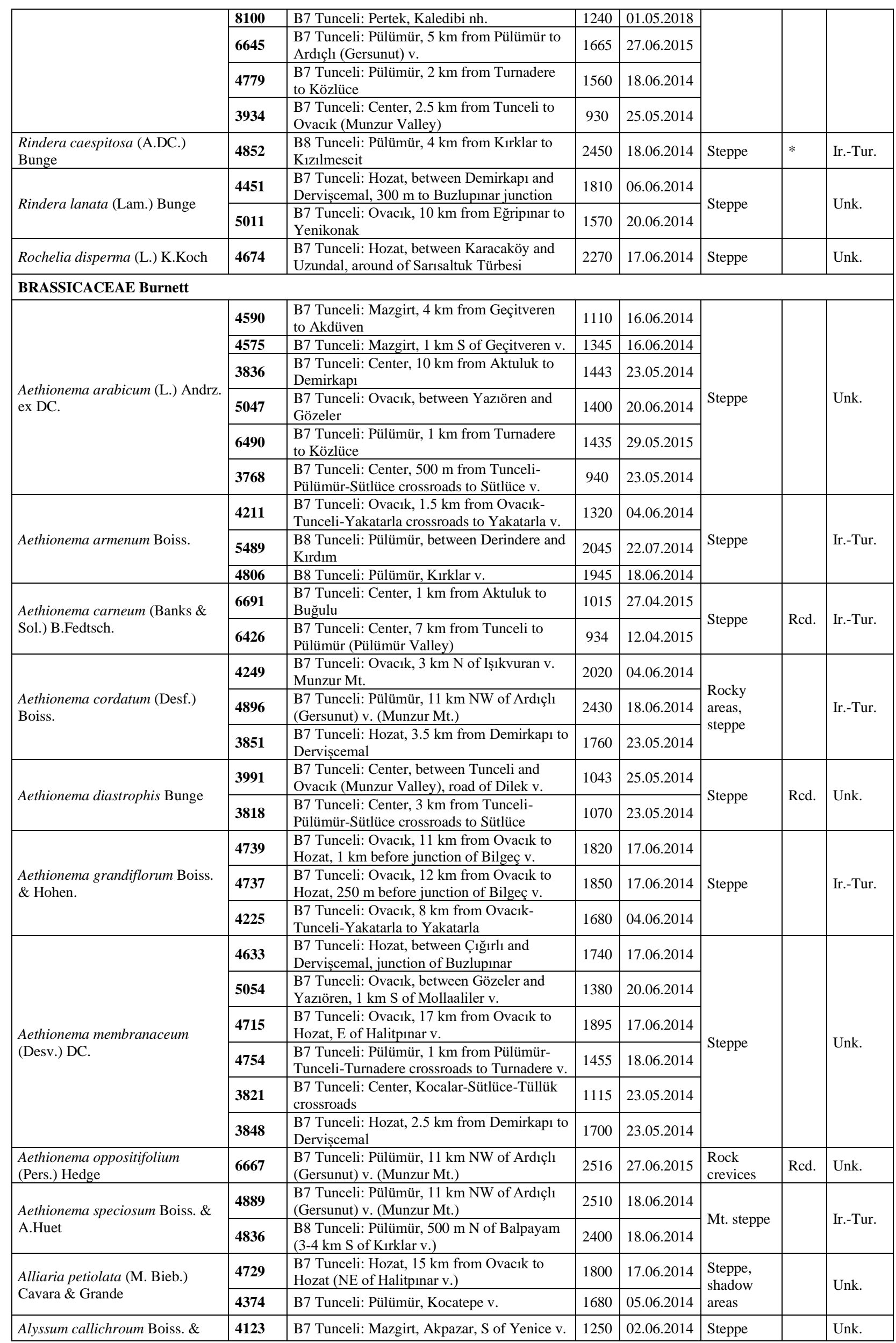




\begin{tabular}{|c|c|c|c|c|c|c|c|}
\hline Balansa & & (N of Şevki mezrası) & & & & & \\
\hline \multirow{2}{*}{$\begin{array}{l}\text { Alyssum condensatum Boiss. \& } \\
\text { Hausskn. subsp. flexibile (Nyar) } \\
\text { T.R.Dudley }\end{array}$} & 5170 & $\begin{array}{l}\text { B7 Tunceli: Çemişgezek, Tahar valley (Ali } \\
\text { Boğazı) }\end{array}$ & 1024 & 29.06 .2014 & \multirow[t]{2}{*}{ Steppe } & & \multirow[t]{2}{*}{ Unk. } \\
\hline & 4381 & B7 Tunceli: Pülümür, Cakırkaya v. & 1750 & 05.06 .2014 & & & \\
\hline $\begin{array}{l}\text { Alyssum contemptum Schott \& } \\
\text { Kotschy }\end{array}$ & 4230 & $\begin{array}{l}\text { B7 Tunceli: Ovacık, } 5.5 \mathrm{~km} \text { from Ovacık- } \\
\text { Tunceli-Yakatarla to Yakatarla }\end{array}$ & 1670 & 04.06 .2014 & Steppe & & Ir.-Tur. \\
\hline \multirow{11}{*}{ Alyssum filiforme Nyár. } & 4703 & $\begin{array}{l}\text { B7 Tunceli: Hozat, between Hozat and } \\
\text { Ovacık, around of Yüceldi v. }\end{array}$ & 1930 & 17.06 .2014 & \multirow{11}{*}{ Steppe } & \multirow{11}{*}{$*$} & \multirow{11}{*}{ Ir.-Tur. } \\
\hline & 4646 & $\begin{array}{l}\text { B7 Tunceli: Hozat, between Karacaköy and } \\
\text { Uzundal, the road of Sarısaltuk Türbesi }\end{array}$ & 1730 & 17.06 .2014 & & & \\
\hline & 3838 & $\begin{array}{l}\text { B7 Tunceli: Center, } 12 \mathrm{~km} \text { from Aktuluk to } \\
\text { Demirkap1 }\end{array}$ & 1465 & 23.05 .2014 & & & \\
\hline & 3928 & B7 Tunceli: Center, Alibaba nh. & 930 & 25.05 .2014 & & & \\
\hline & 5326 & $\begin{array}{l}\text { B7 Tunceli: Nazımiye, } 3 \mathrm{~km} \text { from Nazımiye } \\
\text { to Dereova }\end{array}$ & 1600 & 20.07 .2014 & & & \\
\hline & 4182 & $\begin{array}{l}\text { B7 Tunceli: Nazımiye, } 3 \mathrm{~km} \text { from Nazımiye } \\
\text { to Tunceli }\end{array}$ & 1510 & 03.06 .2014 & & & \\
\hline & 4203 & $\begin{array}{l}\text { B7 Tunceli: Ovacık, } 1.5 \mathrm{~km} \text { from Ovacık- } \\
\text { Tunceli-Yakatarla crossroads to Yakatarla v. }\end{array}$ & 1320 & 04.06 .2014 & & & \\
\hline & 3921 & B7 Tunceli: Pertek, S of Mercimek v. & 1305 & 24.05 .2014 & & & \\
\hline & 3787 & $\begin{array}{l}\text { B7 Tunceli: Center, } 500 \mathrm{~m} \text { from Tunceli- } \\
\text { Pülümür-Sütlüce crossroads to Sütlüce v. }\end{array}$ & 940 & 23.05 .2014 & & & \\
\hline & 3976 & $\begin{array}{l}\text { B7 Tunceli: Center, } 1 \text { km from Tunceli- } \\
\text { Ovacik-Dedeağaç crossroads to Dedeağaç v. } \\
\text { (Munzur Valley) }\end{array}$ & 1060 & 25.05 .2014 & & & \\
\hline & 5490 & B8 Tunceli: Pülümür, W of Kırdım v. & 2070 & 22.07 .2014 & & & \\
\hline \multirow{4}{*}{$\begin{array}{l}\text { Alyssum murale Waldst. \& Kit. } \\
\text { subsp. murale var. murale }\end{array}$} & 5907 & $\begin{array}{l}\text { B7 Tunceli: Ovacık, } 2.5 \mathrm{~km} \text { from Tunceli- } \\
\text { Ovacık-Yakatarla crossroads to Yakatarla }\end{array}$ & 1400 & 08.08 .2014 & \multirow{4}{*}{ Steppe } & \multirow{4}{*}{ Rcd. } & \multirow{4}{*}{ Unk. } \\
\hline & 5044 & $\begin{array}{l}\text { B7 Tunceli: Ovacık, between Yazı̈ren and } \\
\text { Gözeler }\end{array}$ & 1400 & 20.06 .2014 & & & \\
\hline & 5587 & $\begin{array}{l}\text { B7 Tunceli: Pülümür, between Pülümür and } \\
\text { Erzincan, Pülümür Mt. }\end{array}$ & 1900 & 22.07 .2014 & & & \\
\hline & 4219 & $\begin{array}{l}\text { B7 Tunceli: Ovacık, } 5 \mathrm{~km} \text { from Ovacık- } \\
\text { Tunceli-Yakatarla to Yakatarla }\end{array}$ & 1620 & 04.06 .2014 & & & \\
\hline $\begin{array}{l}\text { Alyssum murale Waldst. \& Kit. } \\
\text { subsp. murale var. alpinum Boiss. } \\
\text { ex Nyár. }\end{array}$ & 4807 & B8 Tunceli: Pülümür, 2 km E of Kırklar v. & 2100 & 18.06 .2014 & Steppe & $*$ & Unk. \\
\hline $\begin{array}{l}\text { Alyssum ochroleucum Boiss. \& } \\
\text { A.Huet }\end{array}$ & 4274 & $\begin{array}{l}\text { B7 Tunceli: Ovacık, } 3 \text { km N of Işıkvuran v. } \\
\text { Munzur Mt. }\end{array}$ & 2120 & 04.06 .2014 & Steppe & $*$ & Ir.-Tur. \\
\hline $\begin{array}{l}\text { Alyssum pateri Nyár. subsp. } \\
\text { pateri }\end{array}$ & 4869 & B8 Tunceli: Pülümür, Şampaşakaraderbent v. & 2080 & 18.06 .2014 & Pastures & $*$ & Ir.-Tur. \\
\hline \multirow{2}{*}{$\begin{array}{l}\text { Alyssum pateri Nyár. subsp. } \\
\text { prostratum (Nyár.) T.R.Dudley }\end{array}$} & 4553 & $\begin{array}{l}\text { B7 Tunceli: Mazgirt, } 1 \mathrm{~km} \text { from Ortaharman } \\
\text { to Sülüntaș }\end{array}$ & 1220 & 16.06 .2014 & \multirow{2}{*}{ Steppe } & \multirow{2}{*}{ * } & \multirow{2}{*}{ Ir.-Tur. } \\
\hline & 5043 & $\begin{array}{l}\text { B7 Tunceli: Ovacık, between Yazı̈ren and } \\
\text { Gözeler }\end{array}$ & 1400 & 20.06 .2014 & & & \\
\hline \multirow{4}{*}{$\begin{array}{l}\text { Alyssum peltarioides Boiss. } \\
\text { subsp. virgatiforme (Nyár.) } \\
\text { T.R.Dudley }\end{array}$} & 5937 & $\begin{array}{l}\text { B7 Tunceli: Ovacık, } 5 \mathrm{~km} \text { from Işıkvuran to } \\
\text { Ağaçpınar }\end{array}$ & 2133 & 08.08 .2014 & \multirow{4}{*}{ Mt. steppe } & \multirow{4}{*}{ * } & \multirow{4}{*}{ Unk. } \\
\hline & 4911 & $\begin{array}{l}\text { B7 Tunceli: Ovacık, } 7 \mathrm{~km} \text { from Işıkvuran to } \\
\text { Ağaçpınar }\end{array}$ & 2110 & 19.06.2014 & & & \\
\hline & 5588 & $\begin{array}{l}\text { B7 Tunceli: Pülümür, between Pülümür and } \\
\text { Erzincan, Pülümür Mt. }\end{array}$ & 1900 & 22.07 .2014 & & & \\
\hline & 4840 & $\begin{array}{l}\text { B8 Tunceli: Pülümür, } 500 \mathrm{~m} \mathrm{~N} \text { of Balpayam } \\
\text { (3-4 km S of Kırklar v.) }\end{array}$ & 2400 & 18.06 .2014 & & & \\
\hline Alyssum sibiricum Willd. & 3896 & $\begin{array}{l}\text { B7 Tunceli: Pertek, } 9 \mathrm{~km} \text { from Pertek to } \\
\text { Çemişgezek, } 2.5 \mathrm{~km} \text { before Singeç bridge }\end{array}$ & 865 & 24.05 .2014 & Steppe & & Unk. \\
\hline \multirow[b]{2}{*}{ Alyssum simplex Rudolph } & 4477 & B7 Tunceli: Hozat, 2 km NE of Akpınar v. & 1350 & 06.06 .2014 & \multirow[b]{2}{*}{ Steppe } & & \multirow[b]{2}{*}{ Unk. } \\
\hline & 3985 & $\begin{array}{l}\text { B7 Tunceli: Center, } 10 \mathrm{~km} \text { from Tunceli to } \\
\text { Ovacık (Munzur Valley) }\end{array}$ & 960 & 25.05 .2014 & & & \\
\hline \multirow{2}{*}{ Alyssum strictum Willd. } & 4412 & $\begin{array}{l}\text { B7 Tunceli: Pülümür, between Dereboyu and } \\
\text { Sağlamtaş }\end{array}$ & 1610 & 05.06 .2014 & \multirow{2}{*}{ Steppe } & & \multirow{2}{*}{ Ir.-Tur. } \\
\hline & 4347 & $\begin{array}{l}\text { B7 Tunceli: Pülümür, } 2.5 \mathrm{~km} \text { from Kocatepe } \\
\text { to Sarıül, near to Sarıül junction }\end{array}$ & 1970 & 05.06 .2014 & & & \\
\hline \multirow{2}{*}{ Alyssum strigosum Banks \& Sol. } & 6418 & $\begin{array}{l}\text { B7 Tunceli: Center, Örenönü Nature Park } \\
\text { (Pülümür Valley) }\end{array}$ & 920 & 12.04 .2015 & & & \\
\hline & 4020 & $\begin{array}{l}\text { B7 Tunceli: Center, } 26 \mathrm{~km} \text { from Tunceli to } \\
\text { Ovacık (Munzur Valley) }\end{array}$ & 1060 & 26.05 .2014 & Steppe & & Unk. \\
\hline & 5938 & $\begin{array}{l}\text { B7 Tunceli: Ovacık, } 5 \mathrm{~km} \text { from Işıkvuran to } \\
\text { Ağaçpınar }\end{array}$ & 2133 & 08.08 .2014 & & & Unt \\
\hline Alyssum virgatum Nyár. & 5586 & $\begin{array}{l}\text { B7 Tunceli: Pülümür, between Pülümür and } \\
\text { Erzincan, Pülümür Mt. }\end{array}$ & 1900 & 22.07 .2014 & Steppe & Red. & Unk. \\
\hline Alyssum xanthocarpum Boiss. & 4019 & $\begin{array}{l}\text { B7 Tunceli: Center, } 26 \mathrm{~km} \text { from Tunceli to } \\
\text { Ovacik (Munzur Valley) }\end{array}$ & 1060 & 26.05 .2014 & $\begin{array}{l}\text { Steppe } \\
\text { slopes }\end{array}$ & & Unk. \\
\hline $\begin{array}{l}\text { Anchonium elichrysifolium (DC.) } \\
\text { Boiss. }\end{array}$ & 5819 & $\begin{array}{l}\text { B7 Tunceli: Ovackk, } 8 \mathrm{~km} \text { from Sarıül to } \\
\text { Yalmanlar, the walk way of Buyer Baba }\end{array}$ & 2680 & 06.08 .2014 & Mt. steppe & & Ir.-Tur. \\
\hline
\end{tabular}




\begin{tabular}{|c|c|c|c|c|c|c|c|}
\hline & & sanctuary & & & & & \\
\hline \multirow{2}{*}{ Arabis alpina $\mathrm{L}$. } & 4675 & $\begin{array}{l}\text { B7 Tunceli: Hozat, between Karacaköy and } \\
\text { Uzundal, around of Sarısaltuk Türbesi }\end{array}$ & 2270 & 17.06 .2014 & \multirow{2}{*}{$\begin{array}{l}\text { Rocky } \\
\text { areas, } \\
\text { steppe }\end{array}$} & & \multirow{2}{*}{ Unk. } \\
\hline & 4262 & $\begin{array}{l}\text { B7 Tunceli: Ovacık, } 3 \text { km N of Işıkvuran v. } \\
\text { Munzur Mt. }\end{array}$ & 2100 & 04.06 .2014 & & & \\
\hline \multirow{2}{*}{$\begin{array}{l}\text { Aubrieta libanotica Boiss. \& } \\
\text { Hohen. }\end{array}$} & 4662 & $\begin{array}{l}\text { B7 Tunceli: Hozat, between Karacaköy and } \\
\text { Uzundal, around of Sarısaltuk Türbesi }\end{array}$ & 2270 & 17.06.2014 & \multirow{2}{*}{$\begin{array}{l}\text { Rock } \\
\text { crevices }\end{array}$} & & \multirow{2}{*}{ Unk. } \\
\hline & 6487 & $\begin{array}{l}\text { B7 Tunceli: Pülümür, } 2 \mathrm{~km} \text { from Turnadere } \\
\text { to Közlüce }\end{array}$ & 1528 & 29.05 .2015 & & & \\
\hline \multirow{2}{*}{$\begin{array}{l}\text { Aurinia rupestris (Sweet) Cullen } \\
\& \text { T.R.Dudley subsp. cyclocarpa } \\
\text { (Boiss.) Cullen \& T.R.Dudley }\end{array}$} & 4280 & $\begin{array}{l}\text { B7 Tunceli: Ovacık, } 3 \text { km N of Işıkvuran v. } \\
\text { Munzur Mt. }\end{array}$ & 2150 & 04.06 .2014 & \multirow{2}{*}{$\begin{array}{l}\text { Rocky } \\
\text { areas, } \\
\text { steppe }\end{array}$} & \multirow{2}{*}{$*$} & \multirow{2}{*}{ Unk. } \\
\hline & 4884 & $\begin{array}{l}\text { B7 Tunceli: Pülümür, } 11 \text { km NW of Ardıçlı } \\
\text { (Gersunut) v. (Munzur Mt.) }\end{array}$ & 2510 & 18.06 .2014 & & & \\
\hline \multirow{2}{*}{$\begin{array}{l}\text { Barbarea auriculata Hausskn. ex } \\
\text { Bornm. }\end{array}$} & 6532 & B7 Tunceli: Ovacık, 1 km W of Ağaçpınar & 1934 & 01.06 .2015 & \multirow[b]{2}{*}{ Riverside } & \multirow{2}{*}{ Rcd. } & \multirow[b]{2}{*}{ Ir.-Tur. } \\
\hline & 6500 & $\begin{array}{l}\text { B7 Tunceli: Pülümür, } 2 \mathrm{~km} \text { from Hiver to } \\
\text { Dağyolu (Pülümür Mt.) }\end{array}$ & 1864 & 29.05 .2015 & & & \\
\hline Barbarea brachycarpa Boiss. & 6526 & $\begin{array}{l}\text { B7 Tunceli: Nazımiye, } 12 \mathrm{~km} \mathrm{NE} \mathrm{of} \\
\text { Büyükyurt (Hakis) v., ( } 5 \mathrm{~km} \mathrm{SE} \mathrm{of} \\
\text { Aşağımarkosör) }\end{array}$ & 1926 & 01.06 .2015 & Riverside & Red. & Ir.-Tur. \\
\hline Capsella rubella Reut. & 4582 & B7 Tunceli: Mazgirt, 1 km E of Geçitveren v. & 1270 & 16.06 .2014 & Steppe & Rcd. & Medit. \\
\hline \multirow{2}{*}{ Cardamine uliginosa M.Bieb. } & 6413 & $\begin{array}{l}\text { B7 Tunceli: Center, Örenönü Nature Park } \\
\text { (Pülümür Valley) }\end{array}$ & 920 & 12.04 .2015 & \multirow{2}{*}{$\begin{array}{l}\text { Wet } \\
\text { meadows }\end{array}$} & \multirow{2}{*}{$*$} & \multirow{2}{*}{ Unk. } \\
\hline & 4861 & $\begin{array}{l}\text { B8 Tunceli: Pülümür, } 4 \text { km from Kırklar to } \\
\text { Kizılmescit }\end{array}$ & 2450 & 18.06 .2014 & & & \\
\hline Clypeola jonthlaspi L. & 4335 & $\begin{array}{l}\text { B7 Tunceli: Pülümür, } 5 \mathrm{~km} \text { from Kocatepe to } \\
\text { Çakırkaya }\end{array}$ & 1520 & 05.06 .2014 & On rocks & Rcd. & Unk. \\
\hline \multirow[b]{2}{*}{$\begin{array}{l}\text { Coluteocarpus vesicaria (L.) } \\
\text { Holmboe }\end{array}$} & 4195 & $\begin{array}{l}\text { B7 Tunceli: Ovacik, } 1.5 \mathrm{~km} \text { from Ovacik- } \\
\text { Tunceli-Yakatarla crossroads to Yakatarla v. }\end{array}$ & 1320 & 04.06 .2014 & \multirow[b]{2}{*}{ Steppe } & \multirow[b]{2}{*}{$*$} & \multirow[b]{2}{*}{ Ir.-Tur. } \\
\hline & 5804 & $\begin{array}{l}\text { B7 Tunceli: Ovacik, } 9.5 \mathrm{~km} \text { from Kocatepe to } \\
\text { Yalmanlar (the walk way of Buyer Baba } \\
\text { sanctuary) }\end{array}$ & 2600 & 06.08 .2014 & & & \\
\hline \multirow{3}{*}{ Conringia clavata Boiss. } & 6442 & B7 Tunceli: Center, Alibaba nh. & 930 & 11.04 .2015 & \multirow{3}{*}{ Steppe } & & \multirow{3}{*}{ Unk. } \\
\hline & 6697 & $\begin{array}{l}\text { B7 Tunceli: Center, between Ambar and } \\
\text { Karakoç }\end{array}$ & 970 & 20.04 .2015 & & & \\
\hline & 4757 & $\begin{array}{l}\text { B7 Tunceli: Pülümür, } 1 \mathrm{~km} \text { from Pülümür- } \\
\text { Tunceli-Turnadere crossroads to Turnadere v. }\end{array}$ & 1455 & 18.06.2014 & & & \\
\hline Conringia orientalis (L.) Dumort & 4591 & $\begin{array}{l}\text { B7 Tunceli: Mazgirt, } 4 \mathrm{~km} \text { from Geçitveren } \\
\text { to Akdüven }\end{array}$ & 1110 & 16.06 .2014 & Steppe & Rcd. & Unk. \\
\hline Crambe orientalis $\mathrm{L}$. & Obs. & $\begin{array}{l}\text { B7 Tunceli: Mazgirt, } 7 \mathrm{~km} \text { from Kızılcık to } \\
\text { Göktepe }\end{array}$ & 935 & 02.06 .2014 & Steppe & & Unk. \\
\hline \multirow{2}{*}{ Draba bruniifolia Steven } & 4892 & $\begin{array}{l}\text { B7 Tunceli: Pülümür, } 11 \text { km NW of Ardıçlı } \\
\text { (Gersunut) v. (Munzur Mt.) }\end{array}$ & 2510 & 18.06 .2014 & \multirow{2}{*}{$\begin{array}{l}\text { Rocky } \\
\text { areas, } \\
\text { steppe }\end{array}$} & & \\
\hline & 4833 & $\begin{array}{l}\text { B8 Tunceli: Pülümür, } 500 \mathrm{~m} \mathrm{~N} \text { of Balpayam } \\
\text { (3-4 km S of Kırklar v.) }\end{array}$ & 2400 & 18.06 .2014 & & & Unk. \\
\hline $\begin{array}{l}\text { Draba nuda (Bélanger) Al- } \\
\text { Shehbaz \& M.Koch }\end{array}$ & 6423 & $\begin{array}{l}\text { B7 Tunceli: Pülümür, } 20 \mathrm{~km} \text { from Pülümür to } \\
\text { Tunceli (Pülümür valley) }\end{array}$ & 1215 & 12.04.2015 & Steppe & & Unk. \\
\hline Draba rigida Willd. & 6396 & $\begin{array}{l}\text { B7 Tunceli: Center, } 12 \mathrm{~km} \text { from Tunceli to } \\
\text { Ovacık (Munzur Valley) }\end{array}$ & 1000 & 11.04.2015 & On rocks & Rcd. & Unk. \\
\hline Draba verna $\mathrm{L}$. & 6419 & $\begin{array}{l}\text { B7 Tunceli: Center, Örenönü Nature Park } \\
\text { (Pülümür Valley) }\end{array}$ & 920 & 12.04 .2015 & Meadows & & Unk. \\
\hline Eruca vesicaria (L.) Cav. & 3872 & $\begin{array}{l}\text { B7 Tunceli: Pertek, } 5 \mathrm{~km} \text { from Pertek to } \\
\text { Çemişgezek }\end{array}$ & 866 & 24.05 .2014 & Steppe & Rcd. & Unk. \\
\hline Erysimum crassipes Fisch. \& & 4716 & $\begin{array}{l}\text { B7 Tunceli: Ovacık, } 17 \mathrm{~km} \text { from Ovacık to } \\
\text { Hozat, E of Halitpınar v. }\end{array}$ & 1895 & 17.06.2014 & & & \\
\hline C.A.Mey. & 4428 & $\begin{array}{l}\text { B7 Tunceli: Pülümür, } 9 \mathrm{~km} \text { from Pülümür to } \\
\text { Tunceli (Pülümür valley), S of Kangallı v. }\end{array}$ & 1324 & 05.06 .2014 & Steppe & Rcd. & Unk. \\
\hline Erysimum leucanthemum & 5006 & $\begin{array}{l}\text { B7 Tunceli: Ovacık, } 3.5 \mathrm{~km} \text { from Eğripınar to } \\
\text { Yenikonak }\end{array}$ & 1427 & 20.06 .2014 & & & \\
\hline (Steppehan ex Willd.) B.Fedtsch. & 4859 & $\begin{array}{l}\text { B8 Tunceli: Pülümür, } 4 \text { km from Kırklar to } \\
\text { Kızılmescit }\end{array}$ & 2450 & 18.06 .2014 & Steppe & & Unk. \\
\hline Erysimum pycnophyllum J.Gay & 6495 & $\begin{array}{l}\text { B7 Tunceli: Pülümür, } 7 \text { km from Pülümür to } \\
\text { Erzincan (Pülümür Mt.) }\end{array}$ & 1841 & 29.05 .2015 & Steppe & & Ir.-Tur. \\
\hline & 6523 & $\begin{array}{l}\text { B7 Tunceli: Nazımiye, around of Dereova v., } \\
5 \mathrm{~km} \text { SE of Aşağımarkosör }\end{array}$ & 1970 & 01.06 .2015 & & & Euro.- \\
\hline Ei & 4838 & $\begin{array}{l}\text { B8 Tunceli: Pülümür, } 500 \mathrm{~m} \mathrm{~N} \text { of Balpayam } \\
\text { (3-4 km S of Kırklar v.) }\end{array}$ & 2400 & 18.06 .2014 & Steppe & & \\
\hline & 4023 & $\begin{array}{l}\text { B7 Tunceli: Center, } 26 \mathrm{~km} \text { from Tunceli to } \\
\text { Ovacık (Munzur Valley) }\end{array}$ & 1060 & 26.05 .2014 & Steppe, & & \\
\hline $\begin{array}{l}\text { Fibigia clypeata (L.) Medik. } \\
\text { subsp. clypeata var. eriocarpa } \\
\text { (DC.) Post }\end{array}$ & 4049 & $\begin{array}{l}\text { B7 Tunceli: Ovacık, } 46 \mathrm{~km} \text { from Tunceli to } \\
\text { Ovacık, } 12 \mathrm{~km} \text { before Ovacık (Munzur } \\
\text { Valley) }\end{array}$ & 1213 & 26.05 .2014 & $\begin{array}{l}\text { degraded } \\
\text { oak forest, } \\
\text { dried river }\end{array}$ & & Unk. \\
\hline & 4413 & $\begin{array}{l}\text { B7 Tunceli: Pülümür, between Dereboyu and } \\
\text { Sağlamtaş }\end{array}$ & 1610 & 05.06 .2014 & & & \\
\hline
\end{tabular}




\begin{tabular}{|c|c|c|c|c|c|c|c|}
\hline $\begin{array}{l}\text { Heldreichia bupleurifolia } \text { Boiss. } \\
\text { subsp. bupleurifolia }\end{array}$ & 5821 & B7 Tunceli: Ovacık, NE of Buyer Baba Lake & 2745 & 06.08 .2014 & Mt. steppe & & Unk. \\
\hline \multirow{2}{*}{$\begin{array}{l}\text { Hesperis bicuspidata (Willd.) } \\
\text { Poir. }\end{array}$} & 4850 & $\begin{array}{l}\text { B8 Tunceli: Pülümür, } 4 \text { km from Kırklar to } \\
\text { Kızılmescit }\end{array}$ & 2450 & 18.06 .2014 & \multirow[b]{2}{*}{ Steppe } & \multirow{2}{*}{$*$} & \multirow{2}{*}{ Ir.-Tur. } \\
\hline & 4851 & $\begin{array}{l}\text { B8 Tunceli: Pülümür, } 4 \text { km from Kırklar to } \\
\text { Kızılmescit }\end{array}$ & 2450 & 18.06.2014 & & & \\
\hline \multirow{2}{*}{ Hesperis breviscapa Boiss. } & 4914 & $\begin{array}{l}\text { B7 Tunceli: Ovacık, } 7 \mathrm{~km} \text { from Işıkvuran to } \\
\text { Ağaçpınar }\end{array}$ & 2110 & 19.06.2014 & \multirow[b]{2}{*}{ Steppe } & \multirow[b]{2}{*}{$*$} & \multirow[b]{2}{*}{ Ir.-Tur. } \\
\hline & 4331 & $\begin{array}{l}\text { B7 Tunceli: Pülümür, } 6 \mathrm{~km} \text { from Kocatepe to } \\
\text { Çakırkaya }\end{array}$ & 1510 & 05.06 .2014 & & & \\
\hline Hesperis matronalis L. & 4711 & $\begin{array}{l}\text { B7 Tunceli: Hozat, between Hozat and } \\
\text { Ovacık, } 2 \mathrm{~km} \text { from Yüceldi to Aşlica }\end{array}$ & 1970 & 17.06 .2014 & Steppe & & Unk. \\
\hline \multirow{2}{*}{$\begin{array}{l}\text { Hesperis pendula DC. subsp. } \\
\text { campicarpa (Boiss.) Dvorak }\end{array}$} & 4949 & $\begin{array}{l}\text { B7 Tunceli: Ovacık, N of Eskigedik v., } \\
\text { Munzur Mt. }\end{array}$ & 2160 & 19.06.2014 & \multirow{2}{*}{$\begin{array}{l}\text { Rock } \\
\text { crevices, } \\
\text { steppe }\end{array}$} & \multirow{2}{*}{$*$} & \multirow{2}{*}{ E.Medit. } \\
\hline & 4997 & $\begin{array}{l}\text { B7 Tunceli: Ovacık, Ziyaret v., N of Munzur } \\
\text { Gözeleri }\end{array}$ & 1400 & 20.06 .2014 & & & \\
\hline Hesperis persica Boiss. & 4241 & $\begin{array}{l}\text { B7 Tunceli: Ovacık, } 3 \text { km N of Işıkvuran v. } \\
\text { Munzur Mt. }\end{array}$ & 2050 & 04.06 .2014 & Mt. steppe & Red. & Ir.-Tur. \\
\hline \multirow{4}{*}{ Hesperis thyrsoidea Boiss. } & 4189 & $\begin{array}{l}\text { B7 Tunceli: Ovacık, } 1.5 \mathrm{~km} \text { from Ovacık- } \\
\text { Tunceli-Yakatarla crossroads to Yakatarla v. }\end{array}$ & 1320 & 04.06 .2014 & \multirow{4}{*}{ Steppe } & & \multirow{4}{*}{ Ir.-Tur. } \\
\hline & 4734 & $\begin{array}{l}\text { B7 Tunceli: Hozat, } 14 \mathrm{~km} \text { from Ovacık to } \\
\text { Hozat (NE of Halitpınar v.) }\end{array}$ & 1720 & 17.06.2014 & & & \\
\hline & 4386 & $\begin{array}{l}\text { B7 Tunceli: Pülümür, } 1 \text { km from Çakırkaya } \\
\text { and Dereboyu }\end{array}$ & 1800 & 05.06 .2014 & & & \\
\hline & 4414 & $\begin{array}{l}\text { B7 Tunceli: Pülümür, between Dereboyu and } \\
\text { Sağlamtaş }\end{array}$ & 1610 & 05.06 .2014 & & & \\
\hline $\begin{array}{l}\text { Hirschfeldia incana (L.) Lagr.- } \\
\text { Foss }\end{array}$ & 4485 & $\begin{array}{l}\text { B7 Tunceli: Çemişgezek, } 2 \mathrm{~km} \text { from } \\
\text { Bulgurtepe to Payamdüzü }\end{array}$ & 1060 & 06.06 .2014 & Steppe & Red. & Unk. \\
\hline \multirow{2}{*}{ Hornungia petraea (L.) R.Br. } & 6438 & $\begin{array}{l}\text { B7 Tunceli: Nazımiye, } 33 \mathrm{~km} \text { from Tunceli } \\
\text { to Pülümür (Pülümür Valley) }\end{array}$ & 1061 & 11.04.2015 & \multirow{2}{*}{ Steppe } & \multirow{2}{*}{ Rcd. } & \multirow{2}{*}{ Unk. } \\
\hline & 6402 & $\begin{array}{l}\text { B7 Tunceli: Center, } 15 \mathrm{~km} \text { from Tunceli to } \\
\text { Ovacık (Munzur Valley) }\end{array}$ & 995 & 11.04 .2015 & & & \\
\hline \multirow{2}{*}{ Iberis simplex DC. } & 4131 & $\begin{array}{l}\text { B7 Tunceli: Mazgirt, Akpazar, S of Yenice v. } \\
\text { (S of Şevki mezrası) }\end{array}$ & 1270 & 02.06 .2014 & \multirow{2}{*}{ Steppe } & & \multirow{2}{*}{ Unk. } \\
\hline & 4923 & $\begin{array}{l}\text { B7 Tunceli: Ovacık, } 7 \mathrm{~km} \text { from Işıkvuran to } \\
\text { Eskigedik }\end{array}$ & 2170 & 19.06.2014 & & & \\
\hline \multirow{4}{*}{ Isatis candolleana Boiss. } & 4000 & $\begin{array}{l}\text { B7 Tunceli: Center, between Tunceli and } \\
\text { Ovacık (Munzur Valley), road of Dilek v. }\end{array}$ & 995 & 25.05.2014 & \multirow{4}{*}{ Steppe } & \multirow{4}{*}{$*$} & \multirow{4}{*}{ Ir.-Tur. } \\
\hline & 4191 & $\begin{array}{l}\text { B7 Tunceli: Ovacık, } 1.5 \mathrm{~km} \text { from Ovacık- } \\
\text { Tunceli-Yakatarla crossroads to Yakatarla v. }\end{array}$ & 1320 & 04.06 .2014 & & & \\
\hline & 4389 & $\begin{array}{l}\text { B7 Tunceli: Pülümür, 2-3 km from Çakırkaya } \\
\text { and Dereboyu }\end{array}$ & 1830 & 05.06 .2014 & & & \\
\hline & 3801 & $\begin{array}{l}\text { B7 Tunceli: Center, } 2 \mathrm{~km} \text { from Tunceli- } \\
\text { Pülümür-Sütlüce crossroads to Sütlüce }\end{array}$ & 1050 & 23.05.2014 & & & \\
\hline $\begin{array}{l}\text { Isatis cappadocica Desv. subsp. } \\
\text { cappadocica }\end{array}$ & 3853 & $\begin{array}{l}\text { B7 Tunceli: Hozat, } 3.5 \mathrm{~km} \text { from Demirkapı to } \\
\text { Dervişcemal }\end{array}$ & 1760 & 23.05 .2014 & Steppe & & Ir.-Tur. \\
\hline $\begin{array}{l}\text { Isatis cappadocica Desv. subsp. } \\
\text { macrocarpa (Jaub. \& Spach) } \\
\text { P.H.Davis }\end{array}$ & 5025 & $\begin{array}{l}\text { B7 Tunceli: Ovacık, } 10 \mathrm{~km} \text { from Eğripınar to } \\
\text { Yenikonak }\end{array}$ & 1570 & 20.06 .2014 & steppe & & Ir.-Tur. \\
\hline $\begin{array}{l}\text { Isatis cappadocica Desv. subsp. } \\
\text { steveniana (Trautv.) P.H.Davis }\end{array}$ & 5876 & $\begin{array}{l}\text { B7 Tunceli: Nazımiye, } 6 \mathrm{~km} \mathrm{NE} \text { of } \\
\text { Büyükyurt (Hakis) v. }\end{array}$ & 2015 & 07.08 .2014 & Steppe & & Unk. \\
\hline Isatis cochlearis Boiss. & 3841 & $\begin{array}{l}\text { B7 Tunceli: Center, } 12 \mathrm{~km} \text { from Aktuluk to } \\
\text { Demirkap1 }\end{array}$ & 1430 & 23.05 .2014 & Steppe & Red. & Ir.-Tur. \\
\hline & 5092 & B7 Tunceli: Çemişgezek, center & 946 & 21.06 .2014 & & & \\
\hline Isatis glauca Aucher ex Boiss. & 5380 & $\begin{array}{l}\text { B7 Tunceli: Nazımiye, } 2.5 \mathrm{~km} \text { E of Sarıyayla } \\
\text { v. }\end{array}$ & 2000 & 20.07 .2014 & Steppe & Rcd. & Ir.-Tur. \\
\hline & 5275 & $\begin{array}{l}\text { B7 Tunceli: Pertek, } 8 \mathrm{~km} \text { from Pertek to } \\
\text { Tunceli }\end{array}$ & 1300 & 19.07.2014 & & & \\
\hline Isatis spectabilis P.H.Davis & 4908 & $\begin{array}{l}\text { B7 Tunceli: Pülümür, } 2.5 \text { km NW of Ardıçlı } \\
\text { (Gersunut) v. }\end{array}$ & 1913 & 18.06 .2014 & Steppe & $*$ & Ir.-Tur. \\
\hline Isatis tinctoria L. subsp. & 4731 & $\begin{array}{l}\text { B7 Tunceli: Hozat, } 15 \mathrm{~km} \text { from Ovacık to } \\
\text { Hozat (NE of Halitpınar v.) }\end{array}$ & 1800 & 17.06.2014 & & & \\
\hline tomentella (Boiss.) P.H.Davis & 5650 & $\begin{array}{l}\text { B7 Tunceli: Ovacık, } 36 \mathrm{~km} \text { from Tunceli to } \\
\text { Ovacık (Munzur Valley) }\end{array}$ & 1150 & 24.07 .2014 & Steppe & & Unk. \\
\hline Isatis undulata Aucher ex Boiss. & 5591 & $\begin{array}{l}\text { B7 Tunceli: Pülümür, between Pülümür and } \\
\text { Erzincan, Pülümür Mt. }\end{array}$ & 1900 & 22.07 .2014 & Steppe & $\begin{array}{l}* \\
\text { Rcd. }\end{array}$ & Ir.-Tur. \\
\hline $\begin{array}{l}\text { Matthiola longipetala (Vent.) } \\
\text { DC. }\end{array}$ & 3871 & $\begin{array}{l}\text { B7 Tunceli: Pertek, } 5 \mathrm{~km} \text { from Pertek to } \\
\text { Çemişgezek }\end{array}$ & 866 & 24.05 .2014 & Steppe & Rcd. & Unk. \\
\hline Myagrum perfoliatum $\mathrm{L}$. & 4074 & $\begin{array}{l}\text { B7 Tunceli: Mazgirt, between Tunceli and } \\
\text { Kovancilar, around of Kușcu }\end{array}$ & 867 & 02.06 .2014 & Steppe & Rcd. & Unk. \\
\hline & 4297 & $\begin{array}{l}\text { B7 Tunceli: Ovacık, } 7 \mathrm{~km} \text { from Işıkvuran to } \\
\text { Ağaçpınar }\end{array}$ & 2123 & 04.06 .2014 & & & \\
\hline $\begin{array}{l}\text { Pseudosempervivum aucheri } \\
\text { (Boiss.) Pobed. }\end{array}$ & 4326 & $\begin{array}{l}\text { B7 Tunceli: Pülümür, } 6 \mathrm{~km} \text { from Kocatepe to } \\
\text { Çakırkaya }\end{array}$ & 1510 & 05.06 .2014 & Mt. steppe & $*$ & Ir.-Tur. \\
\hline & 4848 & B8 Tunceli: Pülümür, 500 m N of Balpayam & 2400 & 18.06 .2014 & & & \\
\hline
\end{tabular}




\begin{tabular}{|c|c|c|c|c|c|c|c|}
\hline & & (3-4 km S of Kirklar v.) & & & & & \\
\hline $\begin{array}{l}\text { Ricotia aucheri (Boiss.) } \\
\text { B.L.Burtt }\end{array}$ & 4916 & $\begin{array}{l}\text { B7 Tunceli: Ovacık, } 7 \mathrm{~km} \text { from Işıkvuran to } \\
\text { Ağaçpınar }\end{array}$ & 2110 & 19.06.2014 & Steppe & & Ir.-Tur. \\
\hline Sinapis arvensis L. & 3870 & $\begin{array}{l}\text { B7 Tunceli: Pertek, } 5 \mathrm{~km} \text { from Pertek to } \\
\text { Çemişgezek }\end{array}$ & 866 & 24.05 .2014 & Steppe & Rcd. & Unk. \\
\hline \multirow{3}{*}{ Sisymbrium altissimum $\mathrm{L}$. } & 4082 & $\begin{array}{l}\text { B7 Tunceli: Mazgirt, between Tunceli and } \\
\text { Kovancilar, around of Kușcu }\end{array}$ & 867 & 02.06 .2014 & \multirow{3}{*}{$\begin{array}{l}\text { Ruderal, } \\
\text { steppe }\end{array}$} & & \multirow{3}{*}{ Unk. } \\
\hline & 6696 & B7 Tunceli: Center, Alibaba nh. & 920 & \begin{tabular}{|l|}
20.04 .2015 \\
\end{tabular} & & & \\
\hline & 4980 & B7 Tunceli: Center, Gözen (İksor) v. & 1490 & 19.06.2014 & & & \\
\hline Sisymbrium loeselii $\mathrm{L}$. & 5679 & $\begin{array}{l}\text { B7 Tunceli: Center, } 26 \mathrm{~km} \text { from Tunceli to } \\
\text { Ovacık (Munzur Valley) }\end{array}$ & 1075 & 24.07 .2014 & $\begin{array}{l}\text { Ruderal, } \\
\text { steppe }\end{array}$ & & Unk. \\
\hline Strigosella africana (L.) Botsch. & 6462 & $\begin{array}{l}\text { B7 Tunceli: Nazımiye, Pülümür Valley, } 25 \\
\text { km from Tunceli to Pülümür }\end{array}$ & 1035 & 29.05 .2015 & Ruderal & & Unk. \\
\hline \multirow{4}{*}{ Tchihatchewia isatidea Boiss. } & 4239 & $\begin{array}{l}\text { B7 Tunceli: Ovacık, } 18 \text { km from Ovacık- } \\
\text { Tunceli-Iş1kvuran crossroads to Işı1kvuran }\end{array}$ & 1635 & 04.06 .2014 & \multirow{4}{*}{$\begin{array}{l}\text { Steppe, } \\
\text { rocky } \\
\text { areas }\end{array}$} & \multirow{4}{*}{$*$} & \multirow{4}{*}{ Ir.-Tur. } \\
\hline & 3828 & $\begin{array}{l}\text { B7 Tunceli: Center, } 14 \text { km from Tunceli- } \\
\text { Pülümür-Sütlüce crossroads to Çıralı v. (11 } \\
\text { km before Çıralı v.) }\end{array}$ & 1800 & 23.05 .2014 & & & \\
\hline & 3960 & $\begin{array}{l}\text { B7 Tunceli: Center, } 8 \mathrm{~km} \text { from Tunceli to } \\
\text { Ovacık (Munzur valley) }\end{array}$ & 945 & 25.05 .2014 & & & \\
\hline & 3819 & $\begin{array}{l}\text { B7 Tunceli: Center, } 3 \mathrm{~km} \text { from Tunceli- } \\
\text { Pülümür-Sütlüce crossroads to Sütlüce }\end{array}$ & 1070 & 23.05 .2014 & & & \\
\hline \multirow{2}{*}{ Thlaspi arvense $\mathrm{L}$. } & 4359 & $\begin{array}{l}\text { B7 Tunceli: Pülümür, } 2.5 \mathrm{~km} \text { from Kocatepe } \\
\text { to Sarıgül, near to Sarıgül junction }\end{array}$ & 1970 & 05.06 .2014 & \multirow{2}{*}{$\begin{array}{l}\text { Steppe, } \\
\text { degraded } \\
\text { oak forest }\end{array}$} & \multirow{2}{*}{ Rcd. } & \multirow{2}{*}{ Unk. } \\
\hline & 4854 & $\begin{array}{l}\text { B8 Tunceli: Pülümür, } 4 \text { km from Kırklar to } \\
\text { Kızılmescit }\end{array}$ & 2450 & 18.06 .2014 & & & \\
\hline \multirow{3}{*}{$\begin{array}{l}\text { Thlaspi bornmuelleri (Rech.f.) } \\
\text { Hedge }\end{array}$} & 4290 & B7 Tunceli: Ovacık, Işıkvuran v. & 1710 & 04.06 .2014 & \multirow{3}{*}{$\begin{array}{l}\text { Ruderal, } \\
\text { steppe }\end{array}$} & \multirow{3}{*}{$*$} & \multirow{3}{*}{ Ir.-Tur. } \\
\hline & 4385 & $\begin{array}{l}\text { B7 Tunceli: Pülümür, } 1 \mathrm{~km} \text { from Çakırkaya } \\
\text { and Dereboyu }\end{array}$ & 1800 & 05.06 .2014 & & & \\
\hline & 6499 & $\begin{array}{l}\text { B7 Tunceli: Pülümür, } 1.8 \text { km from Hiver to } \\
\text { Dağyolu (Pülümür Mt.) }\end{array}$ & 1891 & 29.05 .2015 & & & \\
\hline Thlaspi huetii Boiss. & 6369 & $\begin{array}{l}\text { B7 Tunceli: Nazımiye, } 31 \mathrm{~km} \text { from Tunceli } \\
\text { to Pülümür (Pülümür Valley) }\end{array}$ & 1048 & 10.02 .2015 & Steppe & Rcd. & Unk. \\
\hline Thlaspi kurdicum Hedge & 4841 & $\begin{array}{l}\text { B8 Tunceli: Pülümür, } 500 \mathrm{~m} \mathrm{~N} \text { of Balpayam } \\
\text { (3-4 km S of Kırklar v.) }\end{array}$ & 2400 & 18.06 .2014 & Mt. steppe & Red. & Ir.-Tur. \\
\hline $\begin{array}{l}\text { Turritis laxa (Sibth. \& Sm.) } \\
\text { Hayek }\end{array}$ & 6522 & $\begin{array}{l}\text { B7 Tunceli: Nazımiye, around of Dereova v., } \\
5 \mathrm{~km} \text { SE of Aşağımarkosör }\end{array}$ & 1937 & 01.06 .2015 & Steppe & Rcd. & Unk. \\
\hline \multicolumn{8}{|l|}{ CAMPANULACEAE Juss. } \\
\hline $\begin{array}{l}\text { Asyneuma amplexicaule (Willd.) } \\
\text { Hand.-Mazz. }\end{array}$ & 4686 & B7 Tunceli: Hozat, $700 \mathrm{~m} \mathrm{~N}$ of Uzundal v. & 1700 & 17.06 .2014 & Steppe & & Unk. \\
\hline $\begin{array}{l}\text { Asyneuma lobelioides (Willd.) } \\
\text { Hand. -Mazz. }\end{array}$ & 4904 & $\begin{array}{l}\text { B7 Tunceli: Pülümür, } 5 \mathrm{~km} \mathrm{NW} \text { of Ardıçlı } \\
\text { (Gersunut) v. }\end{array}$ & 1975 & 18.06 .2014 & Steppe & & Ir.-Tur. \\
\hline Campanula conferta A.DC. & 4641 & $\begin{array}{l}\text { B7 Tunceli: Hozat, between Karacaköy and } \\
\text { Uzundal, the road of Sarısaltuk Türbesi }\end{array}$ & 1730 & 17.06 .2014 & Steppe & & Unk. \\
\hline $\begin{array}{l}\text { Campanula glomerata L. subsp. } \\
\text { hispida (Witasek) Hayek }\end{array}$ & 3843 & $\begin{array}{l}\text { B7 Tunceli: Center, } 12 \mathrm{~km} \text { from Aktuluk to } \\
\text { Demirkap1 }\end{array}$ & 1430 & 23.05 .2014 & Steppe & & $\begin{array}{l}\text { Euro.- } \\
\text { Sib. }\end{array}$ \\
\hline Campanula hedgei P.H.Davis & 6112 & $\begin{array}{l}\text { B7 Tunceli: Pülümür, } 11 \text { km NW of Ardıçlı } \\
\text { (Gersunut) v. (Munzur Mt.) }\end{array}$ & 2516 & 16.08 .2014 & On rocks & $*$ & Unk. \\
\hline $\begin{array}{l}\text { Campanula munzurensis } \\
\text { P.H.Davis }\end{array}$ & 4835 & $\begin{array}{l}\text { B8 Tunceli: Pülümür, } 500 \mathrm{~m} \mathrm{~N} \text { of Balpayam } \\
\text { (3-4 km S of Kırklar v.) }\end{array}$ & 2400 & 18.06 .2014 & Mt. steppe & $*$ & Unk. \\
\hline $\begin{array}{l}\text { Campanula oligosperma } \\
\text { Dambolt }\end{array}$ & 4935 & $\begin{array}{l}\text { B7 Tunceli: Ovacık, } 12 \mathrm{~km} \text { from Işıkvuran to } \\
\text { Eskigedik }\end{array}$ & 1985 & 19.06.2014 & Steppe & $*$ & Ir.-Tur. \\
\hline $\begin{array}{l}\text { Campanula propinqua } \text { Fisch. \& } \\
\text { C.A.Mey }\end{array}$ & 3748 & $\begin{array}{l}\text { B7 Tunceli: Center, } 1 \mathrm{~km} \text { from Kopuzlar to } \\
\text { Yolkonak }\end{array}$ & 1114 & 22.05 .2014 & Steppe & & Unk. \\
\hline $\begin{array}{l}\text { Campanula quercetorum Hub.- } \\
\text { Mor. \& C.Simon }\end{array}$ & 4965 & $\begin{array}{l}\text { B7 Tunceli: Ovacık, N of Eskigedik v., } \\
\text { Munzur Mt. }\end{array}$ & 2160 & 19.06 .2014 & $\begin{array}{l}\text { Rock } \\
\text { crevices }\end{array}$ & $*$ & Ir.-Tur. \\
\hline $\begin{array}{l}\text { Campanula reuteriana } \text { Boiss. \& } \\
\text { Balansa }\end{array}$ & 4581 & B7 Tunceli: Mazgirt, 1 km E of Geçitveren v. & 1270 & 16.06 .2014 & Steppe & & Unk. \\
\hline Campanula sclerotricha Boiss. & 5918 & $\begin{array}{l}\text { B7 Tunceli: Ovacık, } 3 \text { km N of Işıkvuran v. } \\
\text { Munzur Mt. }\end{array}$ & 2005 & 08.08 .2014 & Steppe & & Unk. \\
\hline Campanula stevenii M.Bieb. & 4810 & B8 Tunceli: Pülümür, 2 km E of Kırklar v. & 2100 & 18.06 .2014 & Steppe & & Ir.-Tur. \\
\hline Campanula tridentata Schreb. & 4872 & $\begin{array}{l}\text { B7 Tunceli: Pülümür, } 11 \text { km NW of Ardıçlı } \\
\text { (Gersunut) v. (Munzur Mt.) }\end{array}$ & 2510 & 18.06 .2014 & $\begin{array}{l}\text { Rocky } \\
\text { areas, } \\
\text { steppe } \\
\end{array}$ & Red. & Unk. \\
\hline $\begin{array}{l}\text { Campanula yildirimlii Kit Tan \& } \\
\text { Sorger }\end{array}$ & 5674 & $\begin{array}{l}\text { B7 Tunceli: Center, } 26 \mathrm{~km} \text { from Tunceli to } \\
\text { Ovacık (Munzur Valley) }\end{array}$ & 1075 & 24.07 .2014 & $\begin{array}{l}\text { Rock } \\
\text { crevices }\end{array}$ & $*$ & Unk. \\
\hline $\begin{array}{l}\text { Legousia falcata (Ten.) Fritsch ex } \\
\text { Janch. }\end{array}$ & 6468 & $\begin{array}{l}\text { B7 Tunceli: Nazımiye, Pülümür Valley, } 25 \\
\text { km from Tunceli to Pülümür }\end{array}$ & 1035 & 29.05 .2015 & Oak forest & Rcd. & Medit. \\
\hline Legousia pentagonia (L.) Thell. & 4540 & B7 Tunceli: Mazgirt, $1 \mathrm{~km} \mathrm{~W}$ of Kayacı v. & 985 & 16.06 .2014 & Steppe & & E.Medit. \\
\hline \multicolumn{8}{|l|}{ CANNABACEAE Martinov } \\
\hline \multirow{2}{*}{$\begin{array}{l}\text { Celtis australis L. subsp. } \\
\text { caucasica (WiId.) C.C.Towns. }\end{array}$} & 6233 & $\begin{array}{l}\text { B7 Tunceli: Center, } 10 \mathrm{~km} \text { from Tunceli to } \\
\text { Pülümür (Pülümür Valley) }\end{array}$ & 949 & 05.09 .2014 & \multirow{2}{*}{ Steppe } & \multirow{2}{*}{ Rcd. } & \multirow{2}{*}{ Unk. } \\
\hline & 6236 & $\begin{array}{l}\text { B7 Tunceli: Center, } 14 \mathrm{~km} \text { from Tunceli to } \\
\text { Pülümür (Pülümür Valley) }\end{array}$ & 965 & 05.09 .2014 & & & \\
\hline
\end{tabular}




\begin{tabular}{|c|c|c|c|c|c|c|c|}
\hline Celtis tournefortii Lam. & 6143 & $\begin{array}{l}\text { B7 Tunceli: between Tunceli - Kovancilar, } 1 \\
\text { km after Kizılcık v. }\end{array}$ & 1110 & 26.08 .2014 & Steppe & & Unk. \\
\hline \multicolumn{8}{|l|}{ CAPRIFOLIACEAE Juss. } \\
\hline \multirow{2}{*}{ Centranthus longiflorus Steven } & 4003 & $\begin{array}{l}\text { B7 Tunceli: Center, between Tunceli and } \\
\text { Ovacık (Munzur Valley), road of Dilek v. }\end{array}$ & 995 & 25.05 .2014 & \multirow{2}{*}{ Steppe } & & \multirow{2}{*}{ Ir.-Tur. } \\
\hline & 5417 & $\begin{array}{l}\text { B7 Tunceli: Hozat, } 2 \mathrm{~km} \text { from Dervişcemal } \\
\text { to Hozat }\end{array}$ & 1730 & 21.07.2014 & & & \\
\hline Cephalaria syriaca (L.) Schrad. & 4075 & $\begin{array}{l}\text { B7 Tunceli: Mazgirt, between Tunceli and } \\
\text { Kovancılar, around of Kuşcu }\end{array}$ & 867 & 02.06 .2014 & Ruderal & & Unk. \\
\hline Dipsacus lacinatus L. & 5448 & $\begin{array}{l}\text { B7 Tunceli: Hozat, N of Boydaş v. (Tahar } \\
\text { Valley) }\end{array}$ & 1215 & 21.07.2014 & Steppe & & Unk. \\
\hline \multirow{3}{*}{ Lonicera orientalis Lam. } & 5012 & $\begin{array}{l}\text { B7 Tunceli: Ovacık, } 10 \mathrm{~km} \text { from Eğripınar to } \\
\text { Yenikonak }\end{array}$ & 1570 & 20.06.2014 & \multirow{3}{*}{$\begin{array}{l}\text { Oak forest, } \\
\text { steppe }\end{array}$} & \multirow{3}{*}{$*$} & \multirow{3}{*}{ Unk. } \\
\hline & 5411 & $\begin{array}{l}\text { B7 Tunceli: Hozat, } 9 \mathrm{~km} \text { from Demirkapı to } \\
\text { Dervişcemal }\end{array}$ & 1765 & 21.07.2014 & & & \\
\hline & 6180 & $\begin{array}{l}\text { B7 Tunceli: Center, } 15 \mathrm{~km} \text { from Tunceli- } \\
\text { Pülümür-Sütlüce crossroads to Çıralı v. (10 } \\
\text { km before Çıralı v.) }\end{array}$ & 1844 & 27.08 .2014 & & & \\
\hline Morina persica $\mathrm{L}$. & 5189 & $\begin{array}{l}\text { B7 Tunceli: Çemişgezek, } 5.5 \mathrm{~km} \text { from } \\
\text { Çemişgezek to Alakuş }\end{array}$ & 1296 & 29.06 .2014 & Steppe & Rcd. & Ir.-Tur. \\
\hline $\begin{array}{l}\text { Pterocephalus plumosus (L.) } \\
\text { Coulter }\end{array}$ & 4615 & $\begin{array}{l}\text { B7 Tunceli: Mazgirt, } 8 \mathrm{~km} \text { from Çukur to } \\
\text { Güleç }\end{array}$ & 1365 & 16.06.2014 & Steppe & Rcd. & Unk. \\
\hline $\begin{array}{l}\text { Scabiosa crinita Kotschy \& } \\
\text { Boiss. }\end{array}$ & 4688 & B7 Tunceli: Hozat, $700 \mathrm{~m} \mathrm{~N}$ of Uzundal v. & 1700 & 17.06 .2014 & Steppe & & Ir.-Tur. \\
\hline Scabiosa micrantha Desf. & 4013 & $\begin{array}{l}\text { B7 Tunceli: Center, } 17 \mathrm{~km} \text { from Tunceli to } \\
\text { Ovacik (Munzur Valley) }\end{array}$ & 1015 & 25.05 .2014 & Steppe & & Unk. \\
\hline Scabiosa persica Boiss. & 4077 & $\begin{array}{l}\text { B7 Tunceli: Mazgirt, between Tunceli and } \\
\text { Kovancılar, around of Kuşcu }\end{array}$ & 867 & 02.06 .2014 & Steppe & Red. & Ir.-Tur. \\
\hline Scabiosa rotata $\mathrm{M} . \mathrm{Bieb}$. & 5048 & $\begin{array}{l}\text { B7 Tunceli: Ovacık, between Yazı̈̈ren and } \\
\text { Gözeler }\end{array}$ & 1400 & 20.06 .2014 & Steppe & & Ir.-Tur. \\
\hline \multirow{2}{*}{ Valeriana alliariifolia Adams } & 4938 & $\begin{array}{l}\text { B7 Tunceli: Ovacık, } 4 \mathrm{~km} \text { from Eskigedik to } \\
\text { Işıkvuran }\end{array}$ & 1890 & 19.06.2014 & \multirow{2}{*}{ Riverside } & & \multirow{2}{*}{ Unk. } \\
\hline & 6071 & B8 Tunceli: Pülümür, Hasangazi v. & 1740 & 16.08 .2014 & & & \\
\hline \multirow[b]{2}{*}{ Valeriana sisymbriifolia Vahl } & 4284 & $\begin{array}{l}\text { B7 Tunceli: Ovacık, } 3 \text { km N of Işıkvuran v. } \\
\text { Munzur Mt. }\end{array}$ & 2300 & 04.06 .2014 & \multirow{2}{*}{$\begin{array}{l}\text { Rock } \\
\text { crevices, } \\
\text { steppe }\end{array}$} & & \multirow[b]{2}{*}{ Ir.-Tur. } \\
\hline & 6517 & $\begin{array}{l}\text { B7 Tunceli: Center, } 14 \text { km from Tunceli- } \\
\text { Pülümür-Sütlüce crossroads to Çıralı v. (11 } \\
\text { km before Çıralı v.) }\end{array}$ & 1770 & 01.06 .2015 & & & \\
\hline \multirow{4}{*}{ Valerianella coronata (L.) DC. } & 3680 & $\begin{array}{l}\text { B7 Tunceli: Center, Tunceli - Elazığ road, } \\
700 \text { m after Aktuluk v. }\end{array}$ & 930 & 22.05 .2014 & \multirow{4}{*}{$\begin{array}{l}\text { Field side, } \\
\text { steppe }\end{array}$} & & \multirow{4}{*}{ Unk. } \\
\hline & 6467 & $\begin{array}{l}\text { B7 Tunceli: Nazımiye, Pülümür Valley, } 25 \\
\text { km from Tunceli to Pülümür }\end{array}$ & 1035 & 29.05 .2015 & & & \\
\hline & 3765 & $\begin{array}{l}\text { B7 Tunceli: Pertek, between Beydamı and } \\
\text { Yolkanak }\end{array}$ & 1300 & 22.05 .2014 & & & \\
\hline & 3931 & $\begin{array}{l}\text { B7 Tunceli: Center, } 2.5 \mathrm{~km} \text { from Tunceli to } \\
\text { Ovacık (Munzur Valley) }\end{array}$ & 930 & 25.05 .2014 & & & \\
\hline \multirow[b]{2}{*}{$\begin{array}{l}\text { Valerianella dactylophylla Boiss. } \\
\text { \& Hohen. }\end{array}$} & 3729 & B7 Tunceli: Center, $1 \mathrm{~km} \mathrm{~S}$ of Aktuluk & 930 & 22.05 .2014 & \multirow[b]{2}{*}{ Steppe } & & \multirow[b]{2}{*}{ Ir.-Tur. } \\
\hline & 7520 & $\begin{array}{l}\text { B7 Tunceli: Pertek, } 9 \mathrm{~km} \text { from Pertek to } \\
\text { Tunceli }\end{array}$ & 1511 & 03.06 .2017 & & & \\
\hline \multirow{2}{*}{$\begin{array}{l}\text { Valerianella glomerata Boiss. \& } \\
\text { Balansa }\end{array}$} & $4576 a$ & B7 Tunceli: Mazgirt, $1 \mathrm{~km} \mathrm{~S}$ of Geçitveren v. & 1345 & 16.06 .2014 & \multirow[b]{2}{*}{ Steppe } & \multirow{2}{*}{$\begin{array}{l}* \\
\text { Rcd. }\end{array}$} & \multirow[b]{2}{*}{ Ir.-Tur. } \\
\hline & 7521 & $\begin{array}{l}\text { B7 Tunceli: Pertek, } 9 \mathrm{~km} \text { from Pertek to } \\
\text { Tunceli }\end{array}$ & 1511 & 03.06 .2017 & & & \\
\hline $\begin{array}{l}\text { Valerianella lasiocarpa Steven } \\
\text { ex Betcke }\end{array}$ & $4576 b$ & B7 Tunceli: Mazgirt, 1 km S of Geçitveren v. & 1345 & 16.06 .2014 & Steppe & Rcd. & Ir.-Tur. \\
\hline \multirow[b]{2}{*}{ Valerianella pumila (L.) DC. } & 8102 & B7 Tunceli: Pertek, Kaledibi nh. & 1240 & \begin{tabular}{|l|}
01.05 .2018 \\
\end{tabular} & & & \\
\hline & 6506 & $\begin{array}{l}\text { B7 Tunceli: Center, } 1 \mathrm{~km} \text { from Sarıtaş to } \\
\text { Gözen (İksor), Kalan Valley }\end{array}$ & 1160 & 30.05 .2015 & Steppe & Rcd. & Unk. \\
\hline $\begin{array}{l}\text { Valerianella uncinata (M.Bieb.) } \\
\text { Dufr. }\end{array}$ & 6450 & $\begin{array}{l}\text { B7 Tunceli: Center, } 17 \mathrm{~km} \text { from Tunceli to } \\
\text { Ovacık (Munzur Valley) }\end{array}$ & 1007 & 28.05 .2015 & Steppe & Rcd. & Ir.-Tur. \\
\hline Valerianella vesicaria (L.) & 3677 & $\begin{array}{l}\text { B7 Tunceli: Center, Tunceli - Elazığ road, } \\
700 \text { m after Aktuluk v. }\end{array}$ & 930 & 22.05 .2014 & Steppe & & Unk. \\
\hline Moench & 3722 & B7 Tunceli: Center, $1 \mathrm{~km} \mathrm{~S}$ of Aktuluk & 930 & 22.05 .2014 & & & \\
\hline CARYOPHYLLACEAE JusS. & & & & & & & \\
\hline $\begin{array}{l}\text { Acanthophyllum oppositiflorum } \\
\text { Aytaç }\end{array}$ & 5966 & $\begin{array}{l}\text { B7 Tunceli: Pertek, } 9 \mathrm{~km} \text { from Pertek to } \\
\text { Çemişgezek, } 2.5 \mathrm{~km} \text { before Singeç bridge }\end{array}$ & 875 & 13.08 .2014 & Steppe & $\begin{array}{l}* \\
\text { Rcd. }\end{array}$ & Ir.-Tur. \\
\hline & 6130 & B7 Tunceli: Mazgirt, S of Dedebağ v. & 860 & 26.08 .2014 & & & \\
\hline & 6116 & B7 Tunceli: Pertek, 2 km W of Bulgurtepe v. & 1060 & 17.08 .2014 & & & \\
\hline $\begin{array}{l}\text { Acanthophyllum verticillatum } \\
\text { C.A.Mey. }\end{array}$ & 5281 & $\begin{array}{l}\text { B7 Tunceli: Pertek, } 8 \mathrm{~km} \text { from Pertek to } \\
\text { Tunceli }\end{array}$ & 1300 & 19.07 .2014 & Steppe & Rcd. & Ir.-Tur. \\
\hline & 5693 & $\begin{array}{l}\text { B7 Tunceli: Center, } 10 \mathrm{~km} \text { from Tunceli to } \\
\text { Ovacık (Munzur Valley) }\end{array}$ & 970 & 24.07.2014 & & & \\
\hline Agrostemma githago L. & 3730 & B7 Tunceli: Center, $1 \mathrm{~km} \mathrm{~S}$ of Aktuluk & 930 & 22.05 .2014 & $\begin{array}{l}\text { Field side, } \\
\text { steppe }\end{array}$ & Red. & Unk. \\
\hline
\end{tabular}




\begin{tabular}{|c|c|c|c|c|c|c|c|}
\hline Arenaria kotschyana Fenzl & 4952 & $\begin{array}{l}\text { B7 Tunceli: Ovacık, N of Eskigedik v., } \\
\text { Munzur Mt. }\end{array}$ & 2160 & 19.06.2014 & $\begin{array}{l}\text { Rock } \\
\text { crevices }\end{array}$ & $*$ & Unk. \\
\hline \multirow{2}{*}{ Arenaria serpyllifolia $\mathrm{L}$. } & 4346 & $\begin{array}{l}\text { B7 Tunceli: Pülümür, } 2.5 \mathrm{~km} \text { from Kocatepe } \\
\text { to Sarıgül, near to Sarı̈ül junction }\end{array}$ & 1970 & 05.06 .2014 & \multirow[t]{2}{*}{ Steppe } & & \multirow[t]{2}{*}{ Unk. } \\
\hline & 4793 & B7 Tunceli: Pülümür, SE of Közlüce v. & 1620 & 18.06 .2014 & & & \\
\hline $\begin{array}{l}\text { Cerastium chlorifolium Fisch. \& } \\
\text { C.A.Mey. }\end{array}$ & 4775 & $\begin{array}{l}\text { B7 Tunceli: Pülümür, } 2 \mathrm{~km} \text { from Turnadere } \\
\text { to Közlüce }\end{array}$ & 1560 & 18.06 .2014 & Steppe & Rcd. & Unk. \\
\hline \multirow{3}{*}{$\begin{array}{l}\text { Cerastium davuricum Fisch. ex } \\
\text { Spreng. }\end{array}$} & 4710 & $\begin{array}{l}\text { B7 Tunceli: Hozat, between Hozat and } \\
\text { Ovacık, } 2 \mathrm{~km} \text { from Yüceldi to Aşlica }\end{array}$ & 1970 & 17.06 .2014 & \multirow{3}{*}{$\begin{array}{l}\text { Wet } \\
\text { meadows, } \\
\text { oak forest }\end{array}$} & & \multirow{3}{*}{$\begin{array}{l}\text { Euro.- } \\
\text { Sib. }\end{array}$} \\
\hline & 4694 & $\begin{array}{l}\text { B7 Tunceli: Hozat, } 4.5 \mathrm{~km} \text { from Uzundal to } \\
\text { Yüceldi }\end{array}$ & 1970 & 17.06.2014 & & & \\
\hline & 3707 & $\begin{array}{l}\text { B7 Tunceli: Center, between Tunceli and } \\
\text { Pertek, junction of Kopuzlar v. }\end{array}$ & 1020 & 22.05 .2014 & & & \\
\hline Cerastium dichotomum $\mathrm{L}$. & 6694 & B7 Tunceli: Center, 2 km E of Buğulu v. & 1173 & 27.04 .2015 & Oak forest & & Unk. \\
\hline Cerastium longifolium Willd. & 4822 & B8 Tunceli: Pülümür, E of Kırklar v. & 2260 & 18.06 .2014 & Steppe & & Ir.-Tur. \\
\hline Cerastium purpurascens Adams & 6674 & B8 Tunceli: Pülümür, 6 km E of Kırklar v. & 2315 & 09.06 .2015 & Steppe & & Unk. \\
\hline Dianthus calocephalus Boiss. & 4785 & B7 Tunceli: Pülümür, SE of Közlüce v. & 1620 & 18.06.2014 & $\begin{array}{l}\text { Steppe } \\
\text { scree }\end{array}$ & & Unk. \\
\hline Dianthus floribundus Boiss. & 5123 & $\begin{array}{l}\text { B7 Tunceli: Pertek, } 3 \mathrm{~km} \text { from Elazığ-Pertek- } \\
\text { Çemişgezek crossroads to Çemişgezek ( } 1 \mathrm{~km} \\
\text { S of Çorovan v.) }\end{array}$ & 870 & 28.06 .2014 & Steppe & & Ir.-Tur. \\
\hline \multirow{4}{*}{ Dianthus orientalis Adams } & 4465 & $\begin{array}{l}\text { B7 Tunceli: Hozat, } 3.5 \mathrm{~km} \text { from Hozat to } \\
\text { Pertek, junction of Kardelen v. }\end{array}$ & 1470 & 06.06 .2014 & \multirow{4}{*}{$\begin{array}{l}\text { Rocky } \\
\text { areas, } \\
\text { steppe }\end{array}$} & & \multirow{4}{*}{ Unk. } \\
\hline & 4197 & $\begin{array}{l}\text { B7 Tunceli: Ovacik, } 1.5 \mathrm{~km} \text { from Ovacık- } \\
\text { Tunceli-Yakatarla crossroads to Yakatarla v. }\end{array}$ & 1320 & 04.06 .2014 & & & \\
\hline & 4956 & $\begin{array}{l}\text { B7 Tunceli: Ovackk, N of Eskigedik v., } \\
\text { Munzur Mt. }\end{array}$ & 2160 & 19.06.2014 & & & \\
\hline & 3958 & $\begin{array}{l}\text { B7 Tunceli: Center, } 8 \mathrm{~km} \text { from Tunceli to } \\
\text { Ovacık (Munzur valley) }\end{array}$ & 945 & 25.05 .2014 & & & \\
\hline $\begin{array}{l}\text { Dianthus tabrisianus Bien. ex } \\
\text { Boiss. }\end{array}$ & 4628 & $\begin{array}{l}\text { B7 Tunceli: Hozat, between Çı̆̆ırlı and } \\
\text { Dervișcemal, junction of Buzlupınar }\end{array}$ & 1740 & 17.06 .2014 & Steppe & Rcd. & Ir.-Tur. \\
\hline Dianthus zederbaueri Vierh. & 5002 & $\begin{array}{l}\text { B7 Tunceli: Ovacık, } 3.5 \mathrm{~km} \text { from Eğripınar to } \\
\text { Yenikonak }\end{array}$ & 1427 & 20.06 .2014 & steppe & $\begin{array}{l}* \\
\text { Rcd. }\end{array}$ & Ir.-Tur. \\
\hline \multirow{2}{*}{$\begin{array}{l}\text { Dianthus zonatus Fenzl var. } \\
\text { aristatus (Boiss.) Reeve }\end{array}$} & 4616 & $\begin{array}{l}\text { B7 Tunceli: Mazgirt, } 5 \mathrm{~km} \text { from Çukur to } \\
\text { Güleç }\end{array}$ & 1430 & 16.06 .2014 & \multirow{2}{*}{$\begin{array}{l}\text { Rocky } \\
\text { areas, } \\
\text { steppe }\end{array}$} & \multirow{2}{*}{ Rcd. } & \multirow{2}{*}{ Unk. } \\
\hline & 4767 & $\begin{array}{l}\text { B7 Tunceli: Pülümür, } 2 \mathrm{~km} \text { from Turnadere } \\
\text { to Közlüce }\end{array}$ & 1575 & 18.06 .2014 & & & \\
\hline \multirow{5}{*}{$\begin{array}{l}\text { Eremogone acutisepala } \\
\text { (Hausskn. ex F.Williams) Ikonn. }\end{array}$} & 5184 & $\begin{array}{l}\text { B7 Tunceli: Çemişgezek, } 5.5 \mathrm{~km} \text { from } \\
\text { Çemişgezek to Alakuş }\end{array}$ & 1296 & 29.06 .2014 & \multirow{5}{*}{ Steppe } & \multirow{5}{*}{$*$} & \multirow{5}{*}{ Ir.-Tur. } \\
\hline & 4618 & $\begin{array}{l}\text { B7 Tunceli: Mazgirt, } 5 \mathrm{~km} \text { from Çukur to } \\
\text { Güleç }\end{array}$ & 1430 & 16.06 .2014 & & & \\
\hline & 4601 & $\begin{array}{l}\text { B7 Tunceli: Mazgirt, } 4 \text { km from Akdüven to } \\
\text { Güleç }\end{array}$ & 1090 & 16.06 .2014 & & & \\
\hline & 5038 & $\begin{array}{l}\text { B7 Tunceli: Ovacık, } 3 \mathrm{~km} \text { from Gözeler to } \\
\text { Yazıören }\end{array}$ & 1483 & 20.06 .2014 & & & \\
\hline & 4213 & $\begin{array}{l}\text { B7 Tunceli: Ovacık, } 1.5 \mathrm{~km} \text { from Ovacık- } \\
\text { Tunceli-Yakatarla crossroads to Yakatarla v. }\end{array}$ & 1320 & 04.06 .2014 & & & \\
\hline $\begin{array}{l}\text { Eremogone blepharophylla } \\
\text { (Boiss.) Ikonn. }\end{array}$ & 4827 & B8 Tunceli: Pülümür, E of Kırklar v. & 2300 & 18.06.2014 & Steppe & Rcd. & Ir.-Tur. \\
\hline \multirow{6}{*}{$\begin{array}{l}\text { Eremogone gypsophiloides (L.) } \\
\text { Fenzl }\end{array}$} & 4449 & $\begin{array}{l}\text { B7 Tunceli: Hozat, between Demirkapı and } \\
\text { Dervişcemal, } 300 \mathrm{~m} \text { to Buzlupınar junction }\end{array}$ & 1810 & 06.06 .2014 & \multirow{6}{*}{ Steppe } & & \multirow{6}{*}{ Ir.-Tur. } \\
\hline & 4450 & $\begin{array}{l}\text { B7 Tunceli: Hozat, between Demirkapı and } \\
\text { Dervişcemal, } 300 \mathrm{~m} \text { to Buzlupınar junction }\end{array}$ & 1810 & 06.06 .2014 & & & \\
\hline & 4648 & $\begin{array}{l}\text { B7 Tunceli: Hozat, between Karacaköy and } \\
\text { Uzundal, the road of Sarisaltuk Türbesi }\end{array}$ & 1730 & 17.06 .2014 & & & \\
\hline & 4740 & $\begin{array}{l}\text { B7 Tunceli: Ovacık, } 11 \mathrm{~km} \text { from Ovacık to } \\
\text { Hozat, } 1 \mathrm{~km} \text { before junction of Bilgeç v. }\end{array}$ & 1820 & 17.06 .2014 & & & \\
\hline & 4770 & $\begin{array}{l}\text { B7 Tunceli: Pülümür, } 2 \mathrm{~km} \text { from Turnadere } \\
\text { to Közlüce }\end{array}$ & 1575 & 18.06 .2014 & & & \\
\hline & 4808 & B8 Tunceli: Pülümür, 2 km E of Kırklar v. & 2100 & 18.06 .2014 & & & \\
\hline $\begin{array}{l}\text { Eremogone ledebouriana (Fenzl) } \\
\text { Ikonn. }\end{array}$ & 5001 & $\begin{array}{l}\text { B7 Tunceli: Ovacık, } 3.5 \mathrm{~km} \text { from Eğripınar to } \\
\text { Yenikonak }\end{array}$ & 1427 & 20.06 .2014 & Steppe & $\begin{array}{l}* \\
\text { Rcd. }\end{array}$ & Ir.-Tur. \\
\hline \multirow{3}{*}{$\begin{array}{l}\text { Eremogone macrantha } \\
\text { (Schischk.) Ikonn. }\end{array}$} & 4282 & $\begin{array}{l}\text { B7 Tunceli: Ovacık, } 3 \text { km N of Işıkvuran v. } \\
\text { Munzur Mt. }\end{array}$ & 2150 & 04.06 .2014 & \multirow{3}{*}{ Steppe } & \multirow{3}{*}{ Red. } & \multirow{3}{*}{ Ir.-Tur. } \\
\hline & 4349 & $\begin{array}{l}\text { B7 Tunceli: Pülümür, } 2.5 \mathrm{~km} \text { from Kocatepe } \\
\text { to Sarıgül, near to Sarıgül junction }\end{array}$ & 1970 & 05.06 .2014 & & & \\
\hline & 4823 & B8 Tunceli: Pülümür, E of Kırklar v. & 2260 & 18.06 .2014 & & & \\
\hline $\begin{array}{l}\text { Eremogone pseudacantholimon } \\
\text { (Bornm.) Holub }\end{array}$ & 4918 & $\begin{array}{l}\text { B7 Tunceli: Ovacık, } 6 \mathrm{~km} \text { from Işıkvuran to } \\
\text { Eskigedik }\end{array}$ & 2250 & 19.06.2014 & Steppe & $*$ & Ir.-Tur. \\
\hline \multirow{4}{*}{ Gypsophila aucheri Boiss. } & 5193 & $\begin{array}{l}\text { B7 Tunceli: Çemişgezek, between Gülbahçe } \\
\text { and Gedikler }\end{array}$ & 1390 & 29.06 .2014 & & & \\
\hline & 4565 & $\begin{array}{l}\text { B7 Tunceli: Mazgirt, } 4 \mathrm{~km} \text { from Ortaharman } \\
\text { to Darıkent }\end{array}$ & 1140 & 16.06.2014 & Steppe & $*$ & Ir.-Tur. \\
\hline & 5390 & B7 Tunceli: Nazımiye, N of Yayıkağıl v. & 1794 & 20.07 .2014 & & & \\
\hline & 5274 & B7 Tunceli: Pertek, $7 \mathrm{~km}$ from Pertek to & 1327 & 19.07 .2014 & & & \\
\hline
\end{tabular}




\begin{tabular}{|c|c|c|c|c|c|c|c|}
\hline & & Tunceli & & & & & \\
\hline & 5571 & B8 Tunceli: Pülümür, 2 km N of Elmalı & 1400 & 22.07 .2014 & & & \\
\hline \multirow[t]{2}{*}{ Gypsophila briquetiana Schischk. } & 5811 & $\begin{array}{l}\text { B7 Tunceli: Ovacık, } 8 \mathrm{~km} \text { from Sarı̈ül to } \\
\text { Yalmanlar, the walk way of Buyer Baba } \\
\text { sanctuary }\end{array}$ & 2680 & 06.08 .2014 & \multirow{2}{*}{$\begin{array}{l}\text { Rock } \\
\text { crevices }\end{array}$} & \multirow[t]{2}{*}{$*$} & \multirow[t]{2}{*}{ Ir.-Tur. } \\
\hline & 5924 & $\begin{array}{l}\text { B7 Tunceli: Ovacık, } 3 \text { km N of Işıkvuran v. } \\
\text { Munzur Mt. }\end{array}$ & 2290 & 08.08.2014 & & & \\
\hline Gypsophila hispida Boiss. & 5523 & $\begin{array}{l}\text { B8 Tunceli: Pülümür, } 3.5 \mathrm{~km} \text { from Bardakçı } \\
\text { to Derindere }\end{array}$ & 1746 & 22.07.2014 & $\begin{array}{l}\text { Steppe } \\
\text { scree, } \\
\text { degraded } \\
\text { oak forest }\end{array}$ & & Ir.-Tur. \\
\hline \multirow{3}{*}{ Gypsophila laricina Schreb. } & 5925 & $\begin{array}{l}\text { B7 Tunceli: Ovacık, } 3 \text { km N of Işıkvuran v. } \\
\text { Munzur Mt. }\end{array}$ & 2290 & 08.08.2014 & \multirow{3}{*}{$\begin{array}{l}\text { Steppe, } \\
\text { rocky } \\
\text { areas }\end{array}$} & \multirow{3}{*}{$*$} & \multirow{3}{*}{ Ir.-Tur. } \\
\hline & 6110 & $\begin{array}{l}\text { B7 Tunceli: Pülümür, } 9 \text { km NW of Ardıçlı } \\
\text { (Gersunut) v. (Munzur Mt.) }\end{array}$ & 2115 & 16.08.2014 & & & \\
\hline & 6094 & B8 Tunceli: Pülümür, 5 km E of Kırklar v. & 2400 & 16.08 .2014 & & & \\
\hline $\begin{array}{l}\text { Gypsophila munzurensis } \\
\text { Armağan }\end{array}$ & 5645 & $\begin{array}{l}\text { B7 Tunceli: Ovac1k, } 30 \mathrm{~km} \text { from Tunceli to } \\
\text { Ovacık (Munzur Valley) }\end{array}$ & 1080 & 24.07 .2014 & Steppe & & Ir.-Tur. \\
\hline Gypsophila ruscifolia Boiss. & 4508 & B7 Tunceli: Mazgirt, $1 \mathrm{~km} \mathrm{~W}$ of Kayacı v. & 990 & 16.06 .2014 & Steppe & & Ir.-Tur. \\
\hline Gypsophila venusta Fenzl & 4085 & $\begin{array}{l}\text { B7 Tunceli: Mazgirt, between Tunceli and } \\
\text { Kovancilar, around of Kușcu }\end{array}$ & 867 & 02.06 .2014 & Steppe & Rcd. & Ir.-Tur. \\
\hline Herniaria hirsuta $\mathrm{L}$. & 3888 & $\begin{array}{l}\text { B7 Tunceli: Pertek, } 9 \mathrm{~km} \text { from Pertek to } \\
\text { Çemişgezek, } 2.5 \mathrm{~km} \text { before Singeç bridge }\end{array}$ & 865 & 24.05 .2014 & Steppe & Red. & Unk. \\
\hline $\begin{array}{l}\text { Holosteum umbellatum L. var. } \\
\text { glutinosum (M.Bieb.) Gay }\end{array}$ & 6380 & $\begin{array}{l}\text { B7 Tunceli: Center, } 3 \mathrm{~km} \text { from Tunceli to } \\
\text { Ovacık (Munzur valley) }\end{array}$ & 931 & 27.03.2015 & Steppe & Red. & Unk. \\
\hline $\begin{array}{l}\text { Holosteum umbellatum L. var. } \\
\text { umbellatum }\end{array}$ & 6432 & $\begin{array}{l}\text { B7 Tunceli: Center, } 7 \mathrm{~km} \text { from Tunceli to } \\
\text { Pülümür (Pülümür Valley) }\end{array}$ & 934 & 12.04.2015 & Steppe & Red. & Unk. \\
\hline $\begin{array}{l}\text { Minuartia anatolica (Boiss.) } \\
\text { Woronow var. lanuginosa } \\
\text { McNeill }\end{array}$ & 4842 & $\begin{array}{l}\text { B8 Tunceli: Pülümür, } 500 \mathrm{~m} \mathrm{~N} \text { of Balpayam } \\
\text { (3-4 km S of Kırklar v.) }\end{array}$ & 2400 & 18.06 .2014 & Mt. steppe & $*$ & Ir.-Tur. \\
\hline $\begin{array}{l}\text { Minuartia erythrosepala (Boiss.) } \\
\text { Hand.-Mazz. }\end{array}$ & 4653 & $\begin{array}{l}\text { B7 Tunceli: Hozat, between Karacaköy and } \\
\text { Uzundal, around of Sarısaltuk Türbesi }\end{array}$ & 2270 & 17.06.2014 & Steppe & & Ir.-Tur. \\
\hline \multirow{2}{*}{$\begin{array}{l}\text { Minuartia glandulosa (Boiss. \& } \\
\text { A.Huet) Bornm. }\end{array}$} & 4452 & $\begin{array}{l}\text { B7 Tunceli: Hozat, between Demirkapı and } \\
\text { Dervişcemal, } 300 \mathrm{~m} \text { to Buzlupınar junction }\end{array}$ & 1810 & 06.06 .2014 & \multirow{2}{*}{$\begin{array}{l}\text { Rocky } \\
\text { areas, } \\
\text { steppe }\end{array}$} & \multirow{2}{*}{$*$} & \multirow{2}{*}{ Ir.-Tur. } \\
\hline & 4897 & $\begin{array}{l}\text { B7 Tunceli: Pülümür, } 11 \text { km NW of Ardıçlı } \\
\text { (Gersunut) v. (Munzur Mt.) }\end{array}$ & 2430 & 18.06 .2014 & & & \\
\hline \multirow{3}{*}{$\begin{array}{l}\text { Minuartia hirsuta (M.Bieb.) } \\
\text { Hand.-Mazz. subsp. falcata } \\
\text { (Gris.) Mattf. }\end{array}$} & 4676 & $\begin{array}{l}\text { B7 Tunceli: Hozat, between Karacaköy and } \\
\text { Uzundal, around of Sarısaltuk Türbesi }\end{array}$ & 2270 & 17.06.2014 & \multirow{3}{*}{ Steppe } & \multirow{3}{*}{ Rcd. } & \multirow{3}{*}{ Ir.-Tur. } \\
\hline & 4638 & $\begin{array}{l}\text { B7 Tunceli: Hozat, between Karacaköy and } \\
\text { Uzundal, the road of Sarısaltuk Türbesi }\end{array}$ & 1730 & 17.06.2014 & & & \\
\hline & 4289 & B7 Tunceli: Ovacık, Işıkvuran v. & 1710 & 04.06 .2014 & & & \\
\hline \multirow{3}{*}{$\begin{array}{l}\text { Minuartia juniperina (L.) Maire } \\
\& \text { Petitm. }\end{array}$} & 4654 & $\begin{array}{l}\text { B7 Tunceli: Hozat, between Karacaköy and } \\
\text { Uzundal, around of Sarısaltuk Türbesi }\end{array}$ & 2270 & 17.06.2014 & \multirow{3}{*}{$\begin{array}{l}\text { Rocky } \\
\text { areas, } \\
\text { steppe }\end{array}$} & & \multirow{3}{*}{ Unk. } \\
\hline & 4273 & $\begin{array}{l}\text { B7 Tunceli: Ovacık, } 3 \text { km N of Işıkvuran v. } \\
\text { Munzur Mt. }\end{array}$ & 2120 & 04.06 .2014 & & & \\
\hline & 4365 & $\begin{array}{l}\text { B7 Tunceli: Pülümür, } 3 \mathrm{~km} \text { from Sarıgül to } \\
\text { Yalmanlar }\end{array}$ & 2250 & 05.06 .2014 & & & \\
\hline $\begin{array}{l}\text { Minuartia mesogitana (Boiss.) } \\
\text { Hand.-Mazz. subsp. kotschyana } \\
\text { (Boiss.) McNeill }\end{array}$ & 6461 & $\begin{array}{l}\text { B7 Tunceli: Nazımiye, Pülümür Valley, } 25 \\
\text { km from Tunceli to Pülümür }\end{array}$ & 1035 & 29.05 .2015 & Steppe & Red. & Unk. \\
\hline $\begin{array}{l}\text { Minuartia meyeri (Boiss.) } \\
\text { Bornm. }\end{array}$ & 4363a & $\begin{array}{l}\text { B7 Tunceli: Pülümür, } 2 \mathrm{~km} \text { from Sarıgül to } \\
\text { Yalmanlar }\end{array}$ & 2200 & 05.06.2014 & Steppe & & Ir.-Tur. \\
\hline $\begin{array}{l}\text { Minuartia multinervis (Boiss.) } \\
\text { Bornm. }\end{array}$ & 4656 & $\begin{array}{l}\text { B7 Tunceli: Hozat, between Karacaköy and } \\
\text { Uzundal, around of Sarısaltuk Türbesi }\end{array}$ & 2270 & 17.06.2014 & Steppe & Rcd. & Unk. \\
\hline $\begin{array}{l}\text { Minuartia recurva (All.) Schinz } \\
\text { \& Thell. subsp. oreina (Mattf.) } \\
\text { McNeill }\end{array}$ & 4826 & B8 Tunceli: Pülümür, E of Kırklar v. & 2300 & 18.06.2014 & Steppe & & Unk. \\
\hline $\begin{array}{l}\text { Minuartia rimarum (Boiss. \& } \\
\text { Balansa) Mattf. }\end{array}$ & 5830 & $\begin{array}{l}\text { B7 Tunceli: Ovacik, } 800 \mathrm{~m} \mathrm{NE} \text { of Buyer } \\
\text { Baba Lake }\end{array}$ & 2821 & 06.08 .2014 & $\begin{array}{l}\text { Rock } \\
\text { crevices }\end{array}$ & $*$ & Ir.-Tur. \\
\hline $\begin{array}{l}\text { Minuartia umbellulifera (Boiss.) } \\
\text { McNeill subsp. umbellifera } \text { var. } \\
\text { kurdica } \text { McNeill }\end{array}$ & 4717 & $\begin{array}{l}\text { B7 Tunceli: Ovacık, } 17 \mathrm{~km} \text { from Ovacık to } \\
\text { Hozat, E of Halitpınar v. }\end{array}$ & 1895 & 17.06.2014 & Steppe & $*$ & Ir.-Tur. \\
\hline \multirow{2}{*}{$\begin{array}{l}\text { Paronychia euphratica } \\
\text { (Chaudhri) Chaudhri }\end{array}$} & 4130 & $\begin{array}{l}\text { B7 Tunceli: Mazgirt, Akpazar, S of Yenice v. } \\
\text { (S of Şevki mezrası) }\end{array}$ & 1270 & 02.06 .2014 & \multirow{2}{*}{$\begin{array}{l}\text { Rocky } \\
\text { areas, } \\
\text { steppe }\end{array}$} & \multirow{2}{*}{$\begin{array}{l}* \\
\text { Rcd. }\end{array}$} & \multirow{2}{*}{ Ir.-Tur. } \\
\hline & 5039 & $\begin{array}{l}\text { B7 Tunceli: Ovacık, } 3 \mathrm{~km} \text { from Gözeler to } \\
\text { Yazıören }\end{array}$ & 1483 & 20.06 .2014 & & & \\
\hline \multirow{4}{*}{$\begin{array}{l}\text { Paronychia kurdica Boiss. subsp. } \\
\text { kurdica }\end{array}$} & 5134 & $\begin{array}{l}\text { B7 Tunceli: Çemişgezek, W of Bozağaç } \\
\text { village }\end{array}$ & 1120 & 28.06.2014 & \multirow{4}{*}{ Steppe } & & \multirow{4}{*}{ Unk. } \\
\hline & 4554 & $\begin{array}{l}\text { B7 Tunceli: Mazgirt, } 1 \mathrm{~km} \text { from Ortaharman } \\
\text { to Sülüntaş }\end{array}$ & 1220 & 16.06.2014 & & & \\
\hline & 3834 & $\begin{array}{l}\text { B7 Tunceli: Center, } 10 \mathrm{~km} \text { from Aktuluk to } \\
\text { Demirkap1 }\end{array}$ & 1443 & 23.05.2014 & & & \\
\hline & 4173 & $\begin{array}{l}\text { B7 Tunceli: Nazımiye, } 5 \mathrm{~km} \text { from Nazımiye } \\
\text { to Tunceli }\end{array}$ & 1435 & 03.06.2014 & & & \\
\hline
\end{tabular}




\begin{tabular}{|c|c|c|c|c|c|c|c|}
\hline & 5712 & $\begin{array}{l}\text { B7 Tunceli: Pertek, between Koçpınar and } \\
\text { Çalı̈̈zü }\end{array}$ & 1117 & 25.07 .2014 & & & \\
\hline & 3884 & $\begin{array}{l}\text { B7 Tunceli: Pertek, } 6.5 \mathrm{~km} \text { from Pertek to } \\
\text { Çemişgezek }\end{array}$ & 868 & 24.05 .2014 & & & \\
\hline & 3769 & $\begin{array}{l}\text { B7 Tunceli: Center, } 500 \text { m from Tunceli- } \\
\text { Pülümür-Sütlüce crossroads to Sütlüce v. }\end{array}$ & 940 & 23.05 .2014 & & & \\
\hline & 3817 & $\begin{array}{l}\text { B7 Tunceli: Center, } 3 \mathrm{~km} \text { from Tunceli- } \\
\text { Pülümür-Sütlüce crossroads to Sütlüce }\end{array}$ & 1070 & 23.05 .2014 & & & \\
\hline $\begin{array}{l}\text { Paronychia kurdica Boiss. subsp. } \\
\text { montis-munzur Chaudhri }\end{array}$ & 4408 & $\begin{array}{l}\text { B7 Tunceli: Pülümür, between Dereboyu and } \\
\text { Sağlamtaş }\end{array}$ & 1610 & 05.06 .2014 & $\begin{array}{l}\text { Rock } \\
\text { crevices }\end{array}$ & $*$ & Unk. \\
\hline \multirow{2}{*}{$\begin{array}{l}\text { Petrorhagia alpina (Hablitz) } \\
\text { P.W.Ball \& Heywood }\end{array}$} & 4714 & $\begin{array}{l}\text { B7 Tunceli: Ovacık, } 17 \mathrm{~km} \text { from Ovacık to } \\
\text { Hozat, E of Halitpınar v. }\end{array}$ & 1895 & 17.06 .2014 & \multirow{2}{*}{ Steppe } & \multirow{2}{*}{ Rcd. } & \multirow{2}{*}{ Unk. } \\
\hline & 4221 & $\begin{array}{l}\text { B7 Tunceli: Ovacik, } 5 \mathrm{~km} \text { from Ovacik- } \\
\text { Tunceli-Yakatarla to Yakatarla }\end{array}$ & 1620 & 04.06 .2014 & & & \\
\hline \multirow{3}{*}{$\begin{array}{l}\text { Petrorhagia cretica }(\text { L.) P.W.Ball } \\
\text { \& Heywood }\end{array}$} & 4558 & $\begin{array}{l}\text { B7 Tunceli: Mazgirt, } 1 \mathrm{~km} \text { from Ortaharman } \\
\text { to Sülüntaş }\end{array}$ & 1220 & 16.06 .2014 & \multirow{3}{*}{ Steppe } & & \multirow{3}{*}{ Unk. } \\
\hline & 4509 & B7 Tunceli: Mazgirt, 1 km W of Kayacı v. & 990 & 16.06 .2014 & & & \\
\hline & 5129 & $\begin{array}{l}\text { B7 Tunceli: Pertek, } 3 \text { km from Elazı̆̆-Pertek- } \\
\text { Çemişgezek crossroads to Çemişgezek (1 km } \\
\text { S of Çorovan v.) }\end{array}$ & 870 & 28.06 .2014 & & & \\
\hline \multirow{2}{*}{$\begin{array}{l}\text { Phryna ortegioides (Fisch. \& } \\
\text { C.A.Mey.) Pax \& K.Hoffm. }\end{array}$} & 5974 & $\begin{array}{l}\text { B7 Tunceli: Hozat, } 4.5 \mathrm{~km} \text { from Hozat to } \\
\text { Pertek }\end{array}$ & 1445 & 13.08 .2014 & \multirow{2}{*}{ Steppe } & \multirow{2}{*}{ Rcd. } & \multirow{2}{*}{ Ir.-Tur. } \\
\hline & 6195 & $\begin{array}{l}\text { B7 Tunceli: Hozat, between Karacaköy and } \\
\text { Uzundal, the road of Sarisaltuk Türbesi }\end{array}$ & 1830 & 28.08 .2014 & & & \\
\hline \multirow{2}{*}{ Saponaria glutinosa M.Bieb. } & 3839 & $\begin{array}{l}\text { B7 Tunceli: Center, } 12 \mathrm{~km} \text { from Aktuluk to } \\
\text { Demirkap1 }\end{array}$ & 1465 & 23.05 .2014 & \multirow{2}{*}{$\begin{array}{l}\text { Degraded } \\
\text { oak forest, } \\
\text { steppe }\end{array}$} & & \multirow{2}{*}{ Unk. } \\
\hline & 4026 & $\begin{array}{l}\text { B7 Tunceli: Center, } 26 \mathrm{~km} \text { from Tunceli to } \\
\text { Ovacık (Munzur Valley) }\end{array}$ & 1060 & 26.05 .2014 & & & \\
\hline Saponaria orientalis L. & 5668 & $\begin{array}{l}\text { B7 Tunceli: Ovacık, } 12 \mathrm{~km} \text { from Eğripınar to } \\
\text { Yenikonak }\end{array}$ & 1570 & 24.07 .2014 & $\begin{array}{l}\text { Steppe } \\
\text { scree }\end{array}$ & Rcd. & Unk. \\
\hline \multirow{2}{*}{$\begin{array}{l}\text { Saponaria prostrata Willd. } \\
\text { subsp. anatolica } \text { Hedge }\end{array}$} & 4944 & $\begin{array}{l}\text { B7 Tunceli: Ovacık, N of Eskigedik v., } \\
\text { Munzur Mt. }\end{array}$ & 1915 & 19.06 .2014 & \multirow{2}{*}{ Steppe } & \multirow{2}{*}{$*$} & \multirow{2}{*}{ Ir.-Tur. } \\
\hline & 4323 & $\begin{array}{l}\text { B7 Tunceli: Pülümür, between Kaymaztepe } \\
\text { and Kovuklu }\end{array}$ & 1545 & 05.06 .2014 & & & \\
\hline \multirow{2}{*}{$\begin{array}{l}\text { Saponaria prostrata Willd. } \\
\text { subsp. prostrata }\end{array}$} & 4033 & $\begin{array}{l}\text { B7 Tunceli: Ovacık, } 41 \mathrm{~km} \text { from Tunceli to } \\
\text { Ovacık (Munzur Valley) }\end{array}$ & 1175 & 26.05 .2014 & \multirow[t]{2}{*}{ Steppe } & \multirow[t]{2}{*}{$*$} & \multirow[t]{2}{*}{ Ir.-Tur. } \\
\hline & 3912 & B7 Tunceli: Pertek, SW of Mercimek v. & 1290 & 24.05 .2014 & & & \\
\hline Scleranthus annuus L. & 4746 & B7 Tunceli: Ovacık, 3 km S of Ovacık & 1315 & 17.06 .2014 & Steppe & Rcd. & Unk. \\
\hline Scleranthus uncinatus Schur & 4569 & B7 Tunceli: Mazgirt, $1 \mathrm{~km} \mathrm{~S}$ of Geçitveren v. & 1345 & 16.06 .2014 & Steppe & Rcd. & Unk. \\
\hline Silene ampullata Boiss. & 3830 & $\begin{array}{l}\text { B7 Tunceli: Center, } 15 \text { km from Tunceli- } \\
\text { Pülümür-Sütlüce crossroads to Çıralı v. (10 } \\
\text { km before Çıralı v.) }\end{array}$ & 1850 & 23.05 .2014 & Steppe & & Ir.-Tur. \\
\hline Silene argentea Ledeb. & 4177 & $\begin{array}{l}\text { B7 Tunceli: Nazımiye, } 5 \mathrm{~km} \text { from Nazımiye } \\
\text { to Tunceli }\end{array}$ & 1435 & 03.06 .2014 & Steppe & & Ir.-Tur. \\
\hline \multirow[t]{2}{*}{ Silene arguta Fenzl } & 4454 & $\begin{array}{l}\text { B7 Tunceli: Hozat, between Demirkapı and } \\
\text { Dervişcemal, } 300 \mathrm{~m} \text { to Buzlupınar junction }\end{array}$ & 1810 & 06.06 .2014 & \multirow{2}{*}{$\begin{array}{l}\text { Rocky } \\
\text { areas, } \\
\text { steppe }\end{array}$} & & \multirow[t]{2}{*}{ Ir.-Tur. } \\
\hline & 4816 & B8 Tunceli: Pülümür, 2.8 km E of Kırklar v. & 2120 & 18.06 .2014 & & & \\
\hline $\begin{array}{l}\text { Silene caryophylloides (Poir.) } \\
\text { Otth subsp. caryophylloides }\end{array}$ & 4878 & $\begin{array}{l}\text { B7 Tunceli: Pülümür, } 11 \text { km NW of Ardıçlı } \\
\text { (Gersunut) v. (Munzur Mt.) }\end{array}$ & 2510 & 18.06 .2014 & $\begin{array}{l}\text { Rocky } \\
\text { areas, } \\
\text { steppe } \\
\end{array}$ & & Ir.-Tur. \\
\hline \multirow{3}{*}{$\begin{array}{l}\text { Silene caryophylloides (Poir.) } \\
\text { Otth subsp. subulata (Boiss.) } \\
\text { Coode \& Cullen }\end{array}$} & 4663 & $\begin{array}{l}\text { B7 Tunceli: Hozat, between Karacaköy and } \\
\text { Uzundal, around of Sarısaltuk Türbesi }\end{array}$ & 2270 & 17.06 .2014 & & & \\
\hline & 4664 & $\begin{array}{l}\text { B7 Tunceli: Hozat, between Karacaköy and } \\
\text { Uzundal, around of Sarısaltuk Türbesi }\end{array}$ & 2270 & 17.06 .2014 & On rocks & & Unk. \\
\hline & 4253 & $\begin{array}{l}\text { B7 Tunceli: Ovacık, } 3 \text { km N of Işıkvuran v. } \\
\text { Munzur Mt. }\end{array}$ & 2020 & 04.06 .2014 & & & \\
\hline & 6159 & $\begin{array}{l}\text { B7 Tunceli: } 10 \mathrm{~km} \text { from Pülümür - Erzincan } \\
\text { mainroad to Süleymanuşağı village }\end{array}$ & 2210 & 27.08.2014 & & & \\
\hline & 3926 & B7 Tunceli: Center, Alibaba nh. & 930 & 25.05 .2014 & & & \\
\hline Silene chlorlfolla Sm. & 3858 & B7 Tunceli: Center, S of Kopuzlar v. & 1084 & 24.05 .2014 & Steppe & & Ir.-I ur. \\
\hline & 4196 & $\begin{array}{l}\text { B7 Tunceli: Ovacik, } 1.5 \mathrm{~km} \text { from Ovacik- } \\
\text { Tunceli-Yakatarla crossroads to Yakatarla v. }\end{array}$ & 1320 & 04.06 .2014 & & & \\
\hline Silene compacta Fisch. ex & 4701 & $\begin{array}{l}\text { B7 Tunceli: Hozat, between Hozat and } \\
\text { Ovacık, around of Yüceldi v. }\end{array}$ & 1930 & 17.06 .2014 & Stenne & & $\mathrm{Ur}$ \\
\hline Hornem. & 3952 & $\begin{array}{l}\text { B7 Tunceli: Center, } 5 \mathrm{~km} \text { from Tunceli to } \\
\text { Ovacık (Munzur Valley) }\end{array}$ & 940 & 25.05 .2014 & Steppe & & Unk. \\
\hline & 4277 & $\begin{array}{l}\text { B7 Tunceli: Ovacık, } 3 \text { km N of Işıkvuran v. } \\
\text { Munzur Mt. }\end{array}$ & 2150 & 04.06 .2014 & & & \\
\hline Silene dianthoides Pers. & 4839 & $\begin{array}{l}\text { B8 Tunceli: Pülümür, } 500 \mathrm{~m} \mathrm{~N} \text { of Balpayam } \\
\text { (3-4 km S of Kırklar v.) }\end{array}$ & 2400 & 18.06 .2014 & Mt. steppe & & Ir.-Tur. \\
\hline Silene italica (L.) Pers. & 4298 & $\begin{array}{l}\text { B7 Tunceli: Ovacık, } 7 \text { km from Işıkvuran to } \\
\text { Ağaçpınar }\end{array}$ & 2123 & 04.06 .2014 & Steppe & & Medit. \\
\hline $\begin{array}{l}\text { Silene latifolia Poir. subsp. alba } \\
\text { (Miller) Greuter \& Burdet }\end{array}$ & 5249 & $\begin{array}{l}\text { B7 Tunceli: Nazımiye, Pülümür Valley, } 43 \\
\text { km from Tunceli to Pülümür }\end{array}$ & 1588 & 30.06 .2014 & $\begin{array}{l}\text { Degraded } \\
\text { oak forest, } \\
\text { steppe }\end{array}$ & & Unk. \\
\hline
\end{tabular}




\begin{tabular}{|c|c|c|c|c|c|c|c|}
\hline Silene longipetala Vent. & 4466 & $\begin{array}{l}\text { B7 Tunceli: Hozat, } 3.5 \mathrm{~km} \text { from Hozat to } \\
\text { Pertek, junction of Kardelen v. }\end{array}$ & 1470 & 06.06 .2014 & Steppe & Rcd. & Unk. \\
\hline \multirow[t]{2}{*}{ Silene manissadjianii Freyn } & 4681 & $\begin{array}{l}\text { B7 Tunceli: Hozat, between Karacaköy and } \\
\text { Uzundal, around of Sarısaltuk Türbesi }\end{array}$ & 2270 & 17.06 .2014 & \multirow[t]{2}{*}{ Steppe } & \multirow{2}{*}{ Rcd. } & \multirow[t]{2}{*}{ Ir.-Tur. } \\
\hline & 4809 & B8 Tunceli: Pülümür, 2 km E of Kırklar v. & 2100 & 18.06 .2014 & & & \\
\hline Silene marschallii C.A.Mey. & 4760 & $\begin{array}{l}\text { B7 Tunceli: Pülümür, } 2 \text { km from Turnadere } \\
\text { to Közlüce }\end{array}$ & 1575 & 18.06.2014 & $\begin{array}{l}\text { Rocky } \\
\text { areas, } \\
\text { steppe } \\
\end{array}$ & & Ir.-Tur. \\
\hline \multirow[b]{2}{*}{ Silene montbretiana Boiss. } & 4479 & $\begin{array}{l}\text { B7 Tunceli: Hozat, between Akpınar and } \\
\text { Karabakır }\end{array}$ & 1275 & 06.06 .2014 & \multirow[b]{2}{*}{ Steppe } & & \multirow[b]{2}{*}{ Ir.-Tur. } \\
\hline & 4725 & $\begin{array}{l}\text { B7 Tunceli: Hozat, } 15 \mathrm{~km} \text { from Ovacık to } \\
\text { Hozat (NE of Halitpınar v.) }\end{array}$ & 1800 & 17.06.2014 & & & \\
\hline \multirow{3}{*}{ Silene odontopetala Fenzl } & 4661 & $\begin{array}{l}\text { B7 Tunceli: Hozat, between Karacaköy and } \\
\text { Uzundal, around of Sarısaltuk Türbesi }\end{array}$ & 2270 & 17.06 .2014 & \multirow{3}{*}{$\begin{array}{l}\text { Rock } \\
\text { crevices }\end{array}$} & & \multirow{3}{*}{ Unk. } \\
\hline & 4665 & $\begin{array}{l}\text { B7 Tunceli: Hozat, between Karacaköy and } \\
\text { Uzundal, around of Sarısaltuk Türbesi }\end{array}$ & 2270 & 17.06 .2014 & & & \\
\hline & 3826 & $\begin{array}{l}\text { B7 Tunceli: Center, } 14 \text { km from Tunceli- } \\
\text { Pülümür-Sütlüce crossroads to Çıralı v. (11 } \\
\text { km before Çıralı v.) }\end{array}$ & 1800 & 23.05 .2014 & & & \\
\hline Silene saxatilis Sims & 4724 & $\begin{array}{l}\text { B7 Tunceli: Hozat, } 15 \mathrm{~km} \text { from Ovacık to } \\
\text { Hozat (NE of Halitpınar v.) }\end{array}$ & 1800 & 17.06.2014 & Steppe & Rcd. & Unk. \\
\hline Silene sclerophylla Chowdhuri & 5254 & $\begin{array}{l}\text { B7 Tunceli: Nazımiye, Pülümür Valley, E } \\
\text { slopes of Hilbeşen bridge, across of Ağlayan } \\
\text { Kayalar }\end{array}$ & 1560 & 30.06 .2014 & Steppe & $*$ & Ir.-Tur. \\
\hline \multirow{3}{*}{$\begin{array}{l}\text { Silene spergulifolia (Desf.) } \\
\text { M.Bieb. }\end{array}$} & 4467 & $\begin{array}{l}\text { B7 Tunceli: Hozat, } 3.5 \mathrm{~km} \text { from Hozat to } \\
\text { Pertek, junction of Kardelen v. }\end{array}$ & 1470 & 06.06 .2014 & \multirow{3}{*}{$\begin{array}{l}\text { Rock } \\
\text { crevices }\end{array}$} & & \multirow{3}{*}{ Ir.-Tur. } \\
\hline & 4178 & $\begin{array}{l}\text { B7 Tunceli: Nazımiye, } 5 \mathrm{~km} \text { from Nazımiye } \\
\text { to Tunceli }\end{array}$ & 1435 & 03.06 .2014 & & & \\
\hline & 3827 & $\begin{array}{l}\text { B7 Tunceli: Center, } 14 \text { km from Tunceli- } \\
\text { Pülümür-Sütlüce crossroads to Çıralı v. (11 } \\
\text { km before Çıralı v.) }\end{array}$ & 1800 & 23.05 .2014 & & & \\
\hline $\begin{array}{l}\text { Silene stenobotrys Boiss. \& } \\
\text { Hausskn. }\end{array}$ & 4523 & B7 Tunceli: Mazgirt, 1 km W of Kayacı v. & 990 & 16.06 .2014 & Steppe & & Ir.-Tur. \\
\hline \multirow{6}{*}{ Silene surculosa Hub.-Mor. } & 4917 & $\begin{array}{l}\text { B7 Tunceli: Ovacık, } 6 \mathrm{~km} \text { from Işıkvuran to } \\
\text { Eskigedik }\end{array}$ & 2250 & 19.06.2014 & \multirow{6}{*}{ Steppe } & \multirow{6}{*}{$*$} & \multirow{6}{*}{ Ir.-Tur. } \\
\hline & 4719 & $\begin{array}{l}\text { B7 Tunceli: Ovacık, } 17 \mathrm{~km} \text { from Ovacık to } \\
\text { Hozat, E of Halitpınar v. }\end{array}$ & 1895 & 17.06 .2014 & & & \\
\hline & 4222 & $\begin{array}{l}\text { B7 Tunceli: Ovacık, } 5 \mathrm{~km} \text { from Ovacık- } \\
\text { Tunceli-Yakatarla to Yakatarla }\end{array}$ & 1620 & 04.06 .2014 & & & \\
\hline & 4900 & $\begin{array}{l}\text { B7 Tunceli: Pülümür, } 11 \text { km NW of Ardıçlı } \\
\text { (Gersunut) v. (Munzur Mt.) }\end{array}$ & 2380 & 18.06 .2014 & & & \\
\hline & 4345 & $\begin{array}{l}\text { B7 Tunceli: Pülümür, } 2.5 \mathrm{~km} \text { from Kocatepe } \\
\text { to Sarıgül, near to Sarıgül junction }\end{array}$ & 1970 & 05.06 .2014 & & & \\
\hline & 6496 & $\begin{array}{l}\text { B7 Tunceli: Pülümür, } 7 \text { km from Pülümür to } \\
\text { Erzincan (Pülümür Mt.) }\end{array}$ & 1841 & 29.05 .2015 & & & \\
\hline Silene swertiifolia Boiss. & 4138 & $\begin{array}{l}\text { B7 Tunceli: Mazgirt, between Tunceli and } \\
\text { Kovancilar, junction of K1z1lc1k v. }\end{array}$ & 1060 & 02.06 .2014 & Steppe & & Ir.-Tur. \\
\hline $\begin{array}{l}\text { Silene vulgaris (Moench) Garcke } \\
\text { var. commutata (Guss.) Coode \& } \\
\text { Cullen }\end{array}$ & 4309 & $\begin{array}{l}\text { B7 Tunceli: Ovacık, } 4 \mathrm{~km} \text { from } \\
\text { Aşağıtorunoba to Ağaçpınar }\end{array}$ & 1800 & 04.06 .2014 & Steppe & & Unk. \\
\hline $\begin{array}{l}\text { Silene vulgaris (Moench) Garcke } \\
\text { var. vulgaris }\end{array}$ & 3708 & $\begin{array}{l}\text { B7 Tunceli: Center, between Tunceli and } \\
\text { Pertek, junction of Kopuzlar v. }\end{array}$ & 1020 & 22.05 .2014 & Oak forest & & Unk. \\
\hline Stellaria media (L.) Vill. & 6435 & B7 Tunceli: Center, Alibaba nh. & 930 & 11.04 .2015 & Steppe & Red. & Unk. \\
\hline \multirow{2}{*}{$\begin{array}{l}\text { Telephium oligospermum Steud. } \\
\text { ex Boiss. }\end{array}$} & 6128 & B7 Tunceli: Mazgirt, S of Dedebağ v. & 860 & 26.08 .2014 & \multirow[b]{2}{*}{ Steppe } & & \multirow[b]{2}{*}{ Ir.-Tur. } \\
\hline & 3780 & $\begin{array}{l}\text { B7 Tunceli: Center, } 500 \text { m from Tunceli- } \\
\text { Pülümür-Sütlüce crossroads to Sütlüce v. }\end{array}$ & 940 & 23.05 .2014 & & & \\
\hline $\begin{array}{l}\text { Vaccaria hispanica (Mill.) } \\
\text { Rauschert (=Vaccaria } \\
\text { pyramidata subsp. grandiflora } \\
\text { (Fisch. ex DC.) Hayek) }\end{array}$ & Obs. & $\begin{array}{l}\text { B7 Tunceli: Center, } 500 \text { m from Tunceli- } \\
\text { Pülümür-Sütlüce crossroads to Sütlüce v. }\end{array}$ & 940 & 23.05 .2014 & Steppe & & Unk. \\
\hline Velezia rigida $\mathrm{L}$. & 4516 & B7 Tunceli: Mazgirt, 1 km W of Kayacı v. & 990 & 16.06 .2014 & Steppe & & Unk. \\
\hline \multicolumn{8}{|l|}{ CELASTRACEAE R.Br. } \\
\hline $\begin{array}{l}\text { Euonymus latifolius Mill. subsp. } \\
\text { cauconis Coode \& Cullen }\end{array}$ & 6036 & $\begin{array}{l}\text { B8 Tunceli: Pülümür, } 2 \mathrm{~km} \mathrm{~W} \text { of } \\
\text { Altınhüseyin }\end{array}$ & 1905 & 14.08 .2014 & Oak forest & $\begin{array}{l}* \\
\text { Rcd. }\end{array}$ & Euxine \\
\hline \multirow{3}{*}{$\begin{array}{l}\text { Euonymus latifolius Mill. subsp. } \\
\text { latifolius }\end{array}$} & 6227 & $\begin{array}{l}\text { B7 Tunceli: Center, } 18 \mathrm{~km} \text { from Tunceli to } \\
\text { Ovacık (Munzur Valley) }\end{array}$ & 975 & 04.09 .2014 & \multirow{3}{*}{ Oak forest } & \multirow{3}{*}{ Rcd. } & \multirow{3}{*}{$\begin{array}{l}\text { Euro.- } \\
\text { Sib. }\end{array}$} \\
\hline & 5885 & $\begin{array}{l}\text { B7 Tunceli: Nazımiye, } 8 \mathrm{~km} \mathrm{NE} \text { of } \\
\text { Büyükyurt (Hakis) v. }\end{array}$ & 1963 & 07.08 .2014 & & & \\
\hline & 6296 & B7 Tunceli: Pülümür, E of Ardıçlı v. & 1747 & 20.09 .2014 & & & \\
\hline Parnassia palustris $\mathrm{L}$. & 6023 & $\begin{array}{l}\text { B8 Tunceli: Pülümür, S of Ağaşenliği v., } \\
\text { between Ağaşenliği and Altınhüseyin }\end{array}$ & 1650 & 14.08 .2014 & $\begin{array}{l}\text { Wet } \\
\text { meadows }\end{array}$ & & Unk. \\
\hline \multicolumn{8}{|l|}{ CLEOMACEAE Airy Shaw } \\
\hline Cleome iberica DC. & 3892 & $\begin{array}{l}\text { B7 Tunceli: Pertek, } 9 \mathrm{~km} \text { from Pertek to } \\
\text { Çemişgezek, } 2.5 \mathrm{~km} \text { before Singeç bridge }\end{array}$ & 865 & 24.05 .2014 & Ruderal & Red. & E.Medit. \\
\hline
\end{tabular}




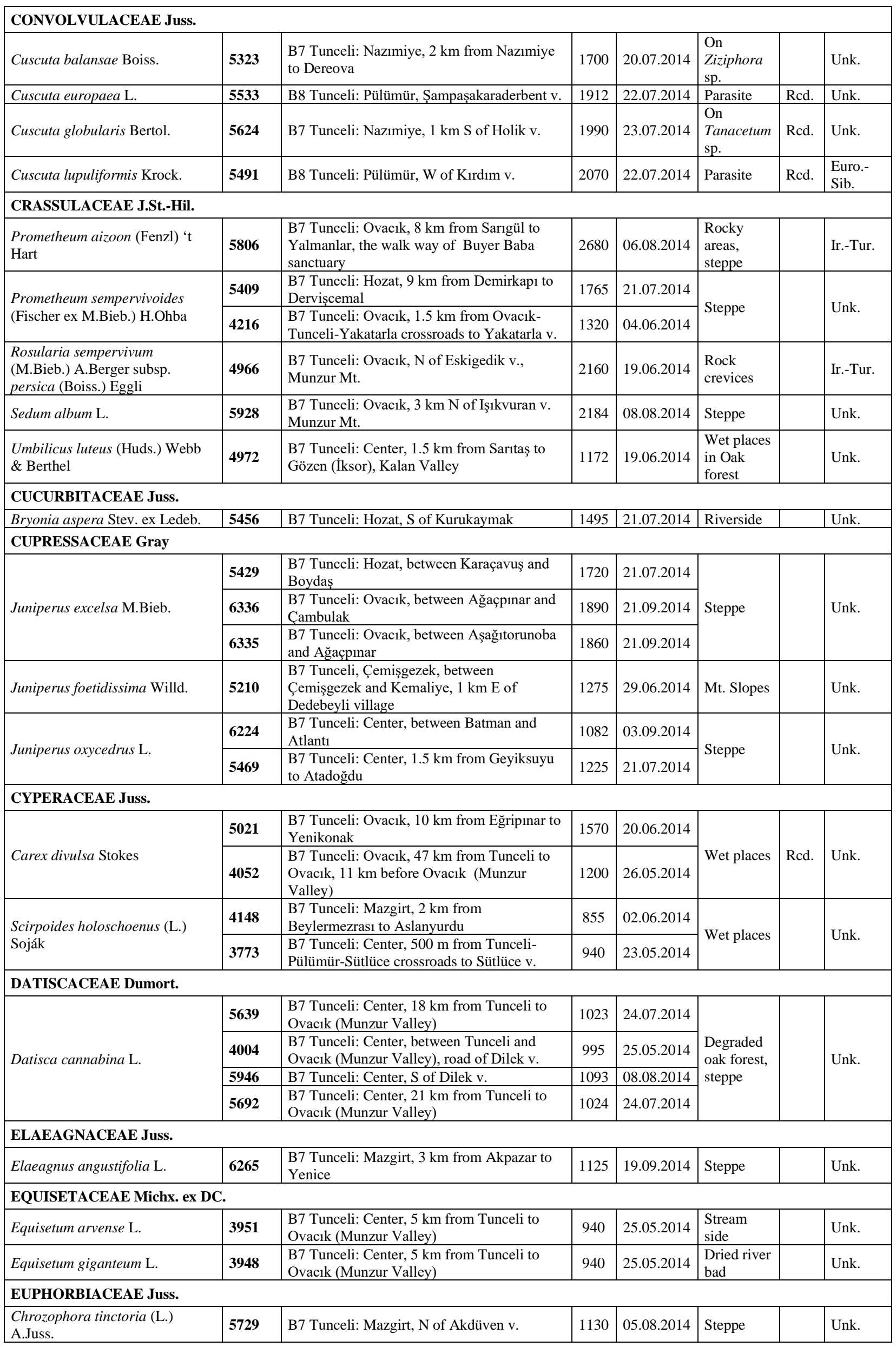




\begin{tabular}{|c|c|c|c|c|c|c|c|}
\hline \multirow[t]{2}{*}{ Euphorbia aleppica L. } & 5088 & $\begin{array}{l}\text { B7 Tunceli: Çemişgezek, between } \\
\text { Çemişgezek and Elazı̆g, } 3 \mathrm{~km} \mathrm{NW} \text { of } \\
\text { Sarıbalta v. }\end{array}$ & 1050 & 21.06 .2014 & \multirow[t]{2}{*}{ Steppe } & \multirow[t]{2}{*}{ Rcd. } & \multirow[t]{2}{*}{ Unk. } \\
\hline & 5714 & $\begin{array}{l}\text { B7 Tunceli: Mazgirt, between Beylermezrası } \\
\text { and Aslanyurdu }\end{array}$ & 860 & 25.07 .2014 & & & \\
\hline \multirow[t]{2}{*}{ Euphorbia chamaesyce L. } & 5734 & $\begin{array}{l}\text { B7 Tunceli: Mazgirt, } 2 \mathrm{~km} \text { from Akdüven to } \\
\text { Güleç }\end{array}$ & 1100 & 05.08 .2014 & \multirow[t]{2}{*}{ Steppe } & & \multirow[t]{2}{*}{ Unk. } \\
\hline & 4506 & B7 Tunceli: Mazgirt, $1 \mathrm{~km} \mathrm{~W}$ of Kayacı v. & 990 & 16.06 .2014 & & & \\
\hline \multirow{5}{*}{$\begin{array}{l}\text { Euphorbia cheiradenia Boiss. \& } \\
\text { Hohen. }\end{array}$} & 4689 & B7 Tunceli: Hozat, $700 \mathrm{~m} \mathrm{~N}$ of Uzundal v. & 1700 & 17.06 .2014 & \multirow{5}{*}{ Steppe } & & \multirow{5}{*}{ Ir.-Tur. } \\
\hline & 5824 & $\begin{array}{l}\text { B7 Tunceli: Ovacık, } 1 \mathrm{~km} \text { NE of Buyer Baba } \\
\text { Lake }\end{array}$ & 2820 & 06.08 .2014 & & & \\
\hline & 3760 & B7 Tunceli: Pertek, 1 km S of Beydamı v. & 1270 & 22.05 .2014 & & & \\
\hline & 4902 & $\begin{array}{l}\text { B7 Tunceli: Pülümür, } 8 \text { km NW of Ardıçlı } \\
\text { (Gersunut) v. }\end{array}$ & 2325 & 18.06 .2014 & & & \\
\hline & 4342 & $\begin{array}{l}\text { B7 Tunceli: Pülümür, between Sarıül and } \\
\text { Kocatepe }\end{array}$ & 1900 & 05.06 .2014 & & & \\
\hline \multirow{3}{*}{ Euphorbia denticulata Lam. } & 4270 & $\begin{array}{l}\text { B7 Tunceli: Ovacık, } 3 \text { km N of Işıkvuran v. } \\
\text { Munzur Mt. }\end{array}$ & 2100 & 04.06 .2014 & \multirow{3}{*}{$\begin{array}{l}\text { Mt. steppe, } \\
\text { degraded } \\
\text { oak forest }\end{array}$} & & \multirow{3}{*}{ Ir.-Tur. } \\
\hline & 4973 & $\begin{array}{l}\text { B7 Tunceli: Center, } 2 \text { km from Sarıtaş to } \\
\text { Gözen (İksor), Kalan Valley }\end{array}$ & 1180 & 19.06 .2014 & & & \\
\hline & 3808 & $\begin{array}{l}\text { B7 Tunceli: Center, } 2 \mathrm{~km} \text { from Tunceli- } \\
\text { Pülümür-Sütlüce crossroads to Sütlüce }\end{array}$ & 1050 & 23.05 .2014 & & & \\
\hline $\begin{array}{l}\text { Euphorbia esula L. subsp. } \\
\text { tommasiniana (Bertol.) } \\
\text { Kuzmanov }\end{array}$ & 4164 & $\begin{array}{l}\text { B7 Tunceli: Nazımiye, } 6.5 \mathrm{~km} \text { from } \\
\text { Nazımiye to Tunceli, } 400 \mathrm{~m} \text { after Geriş v. } \\
\text { junction }\end{array}$ & 1340 & 03.06 .2014 & Steppe & & Unk. \\
\hline \multirow{2}{*}{$\begin{array}{l}\text { Euphorbia falcata } \text { L. subsp. } \\
\text { falcata var. galilaea (Boiss.) } \\
\text { Boiss. }\end{array}$} & 3727 & B7 Tunceli: Center, $1 \mathrm{~km} \mathrm{~S}$ of Aktuluk & 930 & 22.05 .2014 & \multirow[b]{2}{*}{ Steppe } & & \multirow[b]{2}{*}{ Unk. } \\
\hline & 4162 & $\begin{array}{l}\text { B7 Tunceli: Nazımiye, } 6.5 \mathrm{~km} \text { from } \\
\text { Nazımiye to Tunceli, } 400 \mathrm{~m} \text { after Geriş v. } \\
\text { junction }\end{array}$ & 1340 & 03.06 .2014 & & & \\
\hline \multirow{2}{*}{$\begin{array}{l}\text { Euphorbia gaillardotii Boiss. \& } \\
\text { Balanche }\end{array}$} & 4561 & $\begin{array}{l}\text { B7 Tunceli: Mazgirt, } 1 \mathrm{~km} \text { from Ortaharman } \\
\text { to Sülüntaş }\end{array}$ & 1220 & 16.06 .2014 & \multirow{2}{*}{$\begin{array}{l}\text { Steppe, } \\
\text { field side }\end{array}$} & & \multirow{2}{*}{ Ir.-Tur. } \\
\hline & 6222 & $\begin{array}{l}\text { B7 Tunceli: Pertek, betwen Yeniköy and } \\
\text { Pınarlar }\end{array}$ & 1378 & 02.09 .2014 & & & \\
\hline \multirow{3}{*}{ Euphorbia herniariifolia Willd. } & 4659 & $\begin{array}{l}\text { B7 Tunceli: Hozat, between Karacaköy and } \\
\text { Uzundal, around of Sarısaltuk Türbesi }\end{array}$ & 2270 & 17.06.2014 & \multirow{3}{*}{$\begin{array}{l}\text { Mt. steppe, } \\
\text { rocky } \\
\text { areas }\end{array}$} & & \multirow{3}{*}{ Unk. } \\
\hline & 5822 & B7 Tunceli: Ovacık, NE of Buyer Baba Lake & 2745 & 06.08 .2014 & & & \\
\hline & 4887 & $\begin{array}{l}\text { B7 Tunceli: Pülümür, } 11 \mathrm{~km} \mathrm{NW} \text { of Ardıçlı } \\
\text { (Gersunut) v. (Munzur Mt.) }\end{array}$ & 2510 & 18.06 .2014 & & & \\
\hline \multirow{2}{*}{ Euphorbia iberica Boiss. } & 4709 & $\begin{array}{l}\text { B7 Tunceli: Hozat, between Hozat and } \\
\text { Ovacık, } 2 \mathrm{~km} \text { from Yüceldi to Aşlica }\end{array}$ & 1970 & 17.06.2014 & \multirow{2}{*}{ Steppe } & & \multirow{2}{*}{ Ir.-Tur. } \\
\hline & 4682 & $\begin{array}{l}\text { B7 Tunceli: Hozat, between Karacaköy and } \\
\text { Uzundal, around of Sarısaltuk Türbesi }\end{array}$ & 2270 & 17.06.2014 & & & \\
\hline $\begin{array}{l}\text { Euphorbia macrocarpa } \text { Boiss. \& } \\
\text { Buhse }\end{array}$ & 4247 & $\begin{array}{l}\text { B7 Tunceli: Ovacık, } 3 \text { km N of Işskvuran v. } \\
\text { Munzur Mt. }\end{array}$ & 2020 & 04.06 .2014 & Steppe & & Unk. \\
\hline \multirow{7}{*}{ Euphorbia macroclada Boiss. } & 5087 & $\begin{array}{l}\text { B7 Tunceli: Çemişgezek, between } \\
\text { Çemişgezek and Elazı̆g, } 3 \mathrm{~km} \mathrm{NW} \text { of } \\
\text { Sarıbalta v. }\end{array}$ & 1050 & 21.06.2014 & & & \\
\hline & 5325 & $\begin{array}{l}\text { B7 Tunceli: Nazımiye, } 3 \mathrm{~km} \text { from Nazımiye } \\
\text { to Dereova }\end{array}$ & 1600 & 20.07.2014 & & & \\
\hline & 5312 & $\begin{array}{l}\text { B7 Tunceli: Nazımiye, } 5 \mathrm{~km} \text { from Nazımiye } \\
\text { to Tunceli }\end{array}$ & 1447 & 20.07.2014 & Steppe & & Ir.-Tur. \\
\hline & 5898 & $\begin{array}{l}\text { B7 Tunceli: Ovacık, Tunceli-Ovacık- } \\
\text { Yakatarla junction }\end{array}$ & 1240 & 08.08.2014 & & & \\
\hline & 5270 & B7 Tunceli: Pertek, Camikebir nh. & 1100 & 19.07.2014 & & & \\
\hline & 5702 & $\begin{array}{l}\text { B7 Tunceli: Pertek, } 1 \mathrm{~km} \text { from Sumek to } \\
\text { Pinarlar }\end{array}$ & 1177 & 25.07 .2014 & & & \\
\hline & 6029 & B8 Tunceli: Pülümür, 1 km E of Altınhüseyin & 1736 & 14.08 .2014 & & & \\
\hline Funhorbia oriontalis I & 5976 & $\begin{array}{l}\text { B7 Tunceli: Hozat, } 3.2 \mathrm{~km} \text { from Hozat to } \\
\text { Pertek }\end{array}$ & 1440 & 13.08.2014 & Stenne & & $\mathrm{Ir}-\mathrm{Tuu}_{\mathrm{u}}$ \\
\hline Euphorbla orlentals L. & 4022 & $\begin{array}{l}\text { B7 Tunceli: Center, } 26 \mathrm{~km} \text { from Tunceli to } \\
\text { Ovacık (Munzur Valley) }\end{array}$ & 1060 & 26.05.2014 & steppe & & Ir.-1ur. \\
\hline & 4604 & $\begin{array}{l}\text { B7 Tunceli: Mazgirt, } 11 \text { km from Çukur to } \\
\text { Güleç }\end{array}$ & 1230 & 16.06 .2014 & & & \\
\hline Euphorbia szovitsii Fisch. \& & 5029 & $\begin{array}{l}\text { B7 Tunceli: Ovacık, } 10 \mathrm{~km} \text { from Eğripınar to } \\
\text { Yenikonak }\end{array}$ & 1570 & 20.06 .2014 & & & \\
\hline $\begin{array}{l}\text { C.A.Mey. var. kharputensis Azn. } \\
\text { ex M.S.Khan }\end{array}$ & 4995 & $\begin{array}{l}\text { B7 Tunceli: Ovacık, Ziyaret v., N of Munzur } \\
\text { Gözeleri }\end{array}$ & 1400 & 20.06 .2014 & Steppe & & Ir.-Tur. \\
\hline & 5467 & $\begin{array}{l}\text { B7 Tunceli: Center, } 7 \mathrm{~km} \text { from Geyiksuyu to } \\
\text { Taşitlı }\end{array}$ & 1343 & 21.07 .2014 & & & \\
\hline $\begin{array}{l}\text { Euphorbia szovitsii Fisch. \& } \\
\text { C.A.Mey. var. szovitsii }\end{array}$ & 4476 & B7 Tunceli: Hozat, 2 km NE of Akpınar v. & 1350 & 06.06.2014 & Steppe & Red. & Ir.-Tur. \\
\hline FABACEAE Lindl. & & & & & & & \\
\hline $\begin{array}{l}\text { Astragalus cephalotes Banks et } \\
\text { Sol. var. brevicalyx Eig }\end{array}$ & 3756 & $\begin{array}{l}\text { B7 Tunceli: Center, } 1 \mathrm{~km} \text { from Kopuzlar to } \\
\text { Yolkonak }\end{array}$ & 1114 & 22.05 .2014 & Steppe & $\begin{array}{l}* \\
\text { Rcd. }\end{array}$ & Unk. \\
\hline
\end{tabular}




\begin{tabular}{|c|c|c|c|c|c|c|c|}
\hline Astragalus christianus L. & 6547 & $\begin{array}{l}\text { B7 Tunceli: Ovacık, } 9 \mathrm{~km} \text { from } \\
\text { Aşağıtorunoba to Çakmaklı }\end{array}$ & 1800 & 01.06 .2015 & Steppe & Red. & Unk. \\
\hline Astragalus cicer $\mathrm{L}$. & 3840 & $\begin{array}{l}\text { B7 Tunceli: Center, } 12 \mathrm{~km} \text { from Aktuluk to } \\
\text { Demirkap1 }\end{array}$ & 1430 & 23.05 .2014 & $\begin{array}{l}\text { Field side, } \\
\text { steppe }\end{array}$ & Rcd. & Unk. \\
\hline Astragalus cretaceus Boiss. & 3846 & $\begin{array}{l}\text { B7 Tunceli: Hozat, } 2.5 \mathrm{~km} \text { from Demirkapı to } \\
\text { Dervişcemal }\end{array}$ & 1700 & 23.05 .2014 & Steppe & Red. & Ir.-Tur. \\
\hline Astragalus gummifer Labill. & 5068 & $\begin{array}{l}\text { B7 Tunceli: Pertek, } 4.5 \mathrm{~km} \text { from Pertek to } \\
\text { Tunceli, S of Mercimek v. }\end{array}$ & 1307 & 21.06 .2014 & Steppe & Rcd. & Ir.-Tur. \\
\hline Astragalus halicacabus Lam. & 4229 & $\begin{array}{l}\text { B7 Tunceli: Ovacık, } 5.5 \mathrm{~km} \text { from Ovacık- } \\
\text { Tunceli-Yakatarla to Yakatarla }\end{array}$ & 1670 & 04.06 .2014 & Steppe & & Ir.-Tur. \\
\hline Astragalus incertus Ledeb. & 4847 & $\begin{array}{l}\text { B8 Tunceli: Pülümür, } 500 \mathrm{~m} \mathrm{~N} \text { of Balpayam } \\
\text { (3-4 km S of Kırklar v.) }\end{array}$ & 2400 & 18.06 .2014 & Mt. steppe & & Ir.-Tur. \\
\hline Astragalus lamarckii Boiss. & 4642 & $\begin{array}{l}\text { B7 Tunceli: Hozat, between Karacaköy and } \\
\text { Uzundal, the road of Sarısaltuk Türbesi }\end{array}$ & 1730 & 17.06 .2014 & Steppe & $*$ & Ir.-Tur. \\
\hline Astragalus lineatus Lam. & 4362 & $\begin{array}{l}\text { B7 Tunceli: Pülümür, } 2 \mathrm{~km} \text { from Sarı̈ül to } \\
\text { Yalmanlar }\end{array}$ & 2200 & 05.06 .2014 & Steppe & & Unk. \\
\hline \multirow{2}{*}{$\begin{array}{l}\text { Astragalus macrocephalus Willd. } \\
\text { subsp. finitimus (Bunge) } \\
\text { D.F.Chamb. }\end{array}$} & 3798 & $\begin{array}{l}\text { B7 Tunceli: Center, between Tunceli and } \\
\text { Sütlüce, } 1.8 \mathrm{~km} \text { from Sütlüce junction }\end{array}$ & 1030 & 23.05 .2014 & \multirow{2}{*}{ Steppe } & & \multirow{2}{*}{ Ir.-Tur. } \\
\hline & 3757 & $\begin{array}{l}\text { B7 Tunceli: Center, } 1 \mathrm{~km} \text { from Kopuzlar to } \\
\text { Yolkonak }\end{array}$ & 1114 & 22.05 .2014 & & & \\
\hline $\begin{array}{l}\text { Astragalus oocephalus Boiss. } \\
\text { subsp. stachyophorus Hub.-Mor. } \\
\text { \& D.F.Chamb. }\end{array}$ & 4435 & $\begin{array}{l}\text { B7 Tunceli: Center, } 7 \mathrm{~km} \text { from Aktuluk to } \\
\text { Buğulu }\end{array}$ & 1200 & 06.06 .2014 & Steppe & $*$ & Ir.-Tur. \\
\hline \multirow{2}{*}{ Astragalus pendulus DC. } & 4068 & $\begin{array}{l}\text { B7 Tunceli: Ovacık, } 32 \mathrm{~km} \text { from Tunceli to } \\
\text { Ovacık (Munzur Valley) }\end{array}$ & 1110 & 26.05 .2014 & \multirow{2}{*}{ Steppe } & & \multirow{2}{*}{ Ir.-Tur. } \\
\hline & 3845 & $\begin{array}{l}\text { B7 Tunceli: Hozat, } 2.5 \mathrm{~km} \text { from Demirkap1 to } \\
\text { Dervişcemal }\end{array}$ & 1700 & 23.05 .2014 & & & \\
\hline Astragalus scabrifolius Boiss. & 4110 & $\begin{array}{l}\text { B7 Tunceli: Mazgirt, } 7 \mathrm{~km} \text { from Kızılcık to } \\
\text { Göktepe }\end{array}$ & 935 & 02.06 .2014 & Steppe & $\begin{array}{l}* \\
\text { Rcd. }\end{array}$ & Ir.-Tur. \\
\hline Astragalus taochius Woronov & 4443 & $\begin{array}{l}\text { B7 Tunceli: Hozat, between Demirkapı and } \\
\text { Dervişcemal, } 300 \mathrm{~m} \text { to Buzlupınar junction }\end{array}$ & 1810 & 06.06 .2014 & Steppe & $\begin{array}{l}* \\
\text { Rcd. }\end{array}$ & Euxine \\
\hline Astragalus tigridis Boiss. & 3696 & $\begin{array}{l}\text { B7 Tunceli: Center, Tunceli - Elazığ road, } \\
700 \text { m after Aktuluk v. }\end{array}$ & 930 & 22.05 .2014 & $\begin{array}{l}\text { Degraded } \\
\text { oak forest, } \\
\text { steppe }\end{array}$ & & Unk. \\
\hline Astragalus viridis Bunge & 4700 & $\begin{array}{l}\text { B7 Tunceli: Hozat, between Hozat and } \\
\text { Ovacık, around of Yüceldi v. }\end{array}$ & 1930 & 17.06 .2014 & Steppe & Rcd. & Unk. \\
\hline Cicer pinnatifidum Jaub. \& Spach & 4787 & B7 Tunceli: Pülümür, SE of Közlüce v. & 1620 & 18.06 .2014 & Steppe & & Unk. \\
\hline Colutea cilicica Boiss. \& Balansa & 3705 & $\begin{array}{l}\text { B7 Tunceli: Center, between Tunceli and } \\
\text { Pertek, junction of Kopuzlar v. }\end{array}$ & 1020 & 22.05 .2014 & Steppe & & Unk. \\
\hline $\begin{array}{l}\text { Coronilla scorpioides (L.) } \\
\text { W.D.J.Koch }\end{array}$ & 6692 & $\begin{array}{l}\text { B7 Tunceli: Center, } 1 \mathrm{~km} \text { from Aktuluk to } \\
\text { Buğulu }\end{array}$ & 1015 & 27.04 .2015 & Steppe & & Unk. \\
\hline $\begin{array}{l}\text { Cullen jaubertianum (Fenzl) } \\
\text { C.H.Stirt. }\end{array}$ & 4147 & $\begin{array}{l}\text { B7 Tunceli: Mazgirt, between Elazı ̆ and } \\
\text { Tunceli, } 1 \mathrm{~km} \text { E of Beyler mezrası }\end{array}$ & 873 & 02.06 .2014 & Steppe & & Ir.-Tur. \\
\hline $\begin{array}{l}\text { Ebenus haussknechtii Bornm. ex } \\
\text { Hub.-Mor. }\end{array}$ & 3799 & $\begin{array}{l}\text { B7 Tunceli: Center, } 2 \mathrm{~km} \text { from Tunceli- } \\
\text { Pülümür-Sütlüce crossroads to Sütlüce }\end{array}$ & 1050 & 23.05 .2014 & Steppe & $*$ & Ir.-Tur. \\
\hline \multirow{3}{*}{ Galega officinalis L. } & 4699 & $\begin{array}{l}\text { B7 Tunceli: Hozat, } 4.5 \mathrm{~km} \text { from Uzundal to } \\
\text { Yüceldi }\end{array}$ & 1970 & 17.06 .2014 & \multirow{3}{*}{$\begin{array}{l}\text { Degraded } \\
\text { oak forest, } \\
\text { steppe }\end{array}$} & \multirow{3}{*}{ Red. } & \multirow{3}{*}{$\begin{array}{l}\text { Euro.- } \\
\text { Sib. }\end{array}$} \\
\hline & 4623 & $\begin{array}{l}\text { B7 Tunceli: Mazgirt, } 5 \mathrm{~km} \text { from Çukur to } \\
\text { Güleç }\end{array}$ & 1430 & 16.06 .2014 & & & \\
\hline & 4383 & B7 Tunceli: Pülümür, Çakırkaya v. & 1750 & 05.06 .2014 & & & \\
\hline Glycyrrhiza glabra L. & 4084 & $\begin{array}{l}\text { B7 Tunceli: Mazgirt, between Tunceli and } \\
\text { Kovancilar, around of Kuşcu }\end{array}$ & 867 & 02.06 .2014 & $\begin{array}{l}\text { Field side, } \\
\text { steppe }\end{array}$ & & Unk. \\
\hline \multirow{2}{*}{ Hammatolobium lotoides Fenzl } & 5166 & $\begin{array}{l}\text { B7 Tunceli: Çemişgezek, Tahar valley (Ali } \\
\text { Boğazı) }\end{array}$ & 1024 & 29.06 .2014 & \multirow{2}{*}{$\begin{array}{l}\text { Rock } \\
\text { crevices }\end{array}$} & \multirow{2}{*}{ Red. } & \multirow{2}{*}{ E.Medit. } \\
\hline & 3745 & $\begin{array}{l}\text { B7 Tunceli: Center, } 1 \mathrm{~km} \text { from Kopuzlar to } \\
\text { Yolkonak }\end{array}$ & 1114 & 22.05 .2014 & & & \\
\hline \multirow{2}{*}{ Hedysarum erythroleucum Boiss. } & 4881 & $\begin{array}{l}\text { B7 Tunceli: Pülümür, } 11 \text { km NW of Ardıçlı } \\
\text { (Gersunut) v. (Munzur Mt.) }\end{array}$ & 2516 & 18.06 .2014 & \multirow{2}{*}{$\begin{array}{l}\text { Mt. steppe } \\
\text { scree }\end{array}$} & \multirow{2}{*}{ Rcd. } & \multirow{2}{*}{ Ir.-Tur. } \\
\hline & 6666 & $\begin{array}{l}\text { B7 Tunceli: Pülümür, } 11 \text { km NW of Ardıçlı } \\
\text { (Gersunut) v. (Munzur Mt.) }\end{array}$ & 2516 & 27.06 .2015 & & & \\
\hline Hedysarum varium Willd. & 4149 & $\begin{array}{l}\text { B7 Tunceli: Mazgirt, } 2 \mathrm{~km} \text { from } \\
\text { Beylermezrası to Aslanyurdu }\end{array}$ & 855 & 02.06 .2014 & Steppe & & Ir.-Tur. \\
\hline Hippocrepis biflora Spreng. & 3866 & $\begin{array}{l}\text { B7 Tunceli: Pertek, } 5 \mathrm{~km} \text { from Pertek to } \\
\text { Çemişgezek }\end{array}$ & 866 & 24.05 .2014 & Steppe & Rcd. & Unk. \\
\hline \multirow{2}{*}{ Lathyrus chloranthus Boiss. } & 4761 & $\begin{array}{l}\text { B7 Tunceli: Pülümür, } 2 \mathrm{~km} \text { from Turnadere } \\
\text { to Közlüce }\end{array}$ & 1575 & 18.06 .2014 & \multirow{2}{*}{$\begin{array}{l}\text { Dried river } \\
\text { bad }\end{array}$} & & \multirow{2}{*}{ Ir.-Tur. } \\
\hline & 3947 & $\begin{array}{l}\text { B7 Tunceli: Center, } 5 \mathrm{~km} \text { from Tunceli to } \\
\text { Ovacık (Munzur Valley) }\end{array}$ & 940 & 25.05 .2014 & & & \\
\hline Lathyrus roseus Steven & 3844 & $\begin{array}{l}\text { B7 Tunceli: Center, } 13 \mathrm{~km} \text { from Aktuluk to } \\
\text { Demirkap1 }\end{array}$ & 1396 & 23.05 .2014 & $\begin{array}{l}\text { Degraded } \\
\text { oak forest, } \\
\text { steppe } \\
\end{array}$ & & $\begin{array}{l}\text { Hyrc.- } \\
\text { Euxine }\end{array}$ \\
\hline $\begin{array}{l}\text { Lathyrus rotundifolius Willd. } \\
\text { subsp. miniatus (M.Bieb. ex } \\
\text { Steven) P.H.Davis }\end{array}$ & 4045 & $\begin{array}{l}\text { B7 Tunceli: Ovacık, } 44 \mathrm{~km} \text { from Tunceli to } \\
\text { Ovacık, } 14 \mathrm{~km} \text { before Ovacık (Munzur } \\
\text { Valley) }\end{array}$ & 1190 & 26.05 .2014 & Oak forest & & Unk. \\
\hline Lotus aegeus (Griseb.) Boiss. & 4143 & B7 Tunceli: Mazgirt, $1 \mathrm{~km}$ from Kızılcık to & 970 & 02.06 .2014 & Steppe & Rcd. & Unk. \\
\hline
\end{tabular}




\begin{tabular}{|c|c|c|c|c|c|c|c|}
\hline & & Göktepe & & & & & \\
\hline $\begin{array}{l}\text { Lotus gebelia Vent. var. } \\
\text { anthylloides Boiss. }\end{array}$ & 3908 & $\begin{array}{l}\text { B7 Tunceli: Pertek, } 12 \mathrm{~km} \text { from Pertek to } \\
\text { Çemişgezek, around of Singeç bridge }\end{array}$ & 855 & 24.05 .2014 & $\begin{array}{l}\text { Rocky } \\
\text { areas, } \\
\text { steppe }\end{array}$ & $*$ & Ir.-Tur. \\
\hline $\begin{array}{l}\text { Lotus gebelia Vent. var. } \\
\text { hirsutissimus (Ledeb.) Dinsm. }\end{array}$ & 4140 & $\begin{array}{l}\text { B7 Tunceli: Mazgirt, } 1 \mathrm{~km} \text { from Kızılcık to } \\
\text { Göktepe }\end{array}$ & 1132 & 02.06 .2014 & Steppe & & Ir.-Tur. \\
\hline $\begin{array}{l}\text { Medicago fischeriana (Ser.) } \\
\text { Trautv. }\end{array}$ & 3875 & $\begin{array}{l}\text { B7 Tunceli: Pertek, } 5 \mathrm{~km} \text { from Pertek to } \\
\text { Çemişgezek }\end{array}$ & 866 & 24.05 .2014 & Steppe & & Ir.-Tur. \\
\hline Medicago minima (L.) Bartal. & 4171 & $\begin{array}{l}\text { B7 Tunceli: Nazımiye, } 5 \mathrm{~km} \text { from Nazımiye } \\
\text { to Tunceli }\end{array}$ & 1435 & 03.06 .2014 & Steppe & & Unk. \\
\hline Medicago noeana Boiss. & 4104 & $\begin{array}{l}\text { B7 Tunceli: Mazgirt, } 7 \mathrm{~km} \text { from Kızılcık to } \\
\text { Göktepe }\end{array}$ & 935 & 02.06 .2014 & Steppe & Rcd. & Ir.-Tur. \\
\hline Medicago radiata $\mathrm{L}$. & 3835 & $\begin{array}{l}\text { B7 Tunceli: Center, } 10 \mathrm{~km} \text { from Aktuluk to } \\
\text { Demirkap1 }\end{array}$ & 1443 & 23.05 .2014 & Steppe & & Ir.-Tur. \\
\hline Melilotus albus Desr. & 4095 & $\begin{array}{l}\text { B7 Tunceli: Mazgirt, between Tunceli and } \\
\text { Kovancilar, around of Kușcu }\end{array}$ & 867 & 02.06 .2014 & $\begin{array}{l}\text { Ruderal, in } \\
\text { gardens }\end{array}$ & & Unk. \\
\hline Melilotus officinalis (L.) Desr. & 4094 & $\begin{array}{l}\text { B7 Tunceli: Mazgirt, between Tunceli and } \\
\text { Kovancilar, around of Kuşcu }\end{array}$ & 867 & 02.06 .2014 & $\begin{array}{l}\text { Ruderal, in } \\
\text { gardens }\end{array}$ & & Unk. \\
\hline \multirow{3}{*}{ Onobrychis argyrea Boiss. } & 3900 & $\begin{array}{l}\text { B7 Tunceli: Pertek, } 12 \mathrm{~km} \text { from Pertek to } \\
\text { Çemişgezek, around of Singeç bridge }\end{array}$ & 855 & 24.05 .2014 & \multirow{3}{*}{ Steppe } & & \multirow{3}{*}{ Ir.-Tur. } \\
\hline & 3879 & $\begin{array}{l}\text { B7 Tunceli: Pertek, } 5 \mathrm{~km} \text { from Pertek to } \\
\text { Çemişgezek }\end{array}$ & 866 & 24.05 .2014 & & & \\
\hline & 3800 & $\begin{array}{l}\text { B7 Tunceli: Center, } 2 \mathrm{~km} \text { from Tunceli- } \\
\text { Pülümür-Sütlüce crossroads to Sütlüce }\end{array}$ & 1050 & 23.05 .2014 & & & \\
\hline Onobrychis cornuta (L.) Desv. & 4266 & $\begin{array}{l}\text { B7 Tunceli: Ovacık, } 3 \text { km N of Işıkvuran v. } \\
\text { Munzur Mt. }\end{array}$ & 2100 & 04.06 .2014 & Mt. steppe & & Unk. \\
\hline \multirow{2}{*}{ Onobrychis galegifolia Boiss. } & 4567 & $\begin{array}{l}\text { B7 Tunceli: Mazgirt, } 4 \mathrm{~km} \text { from Ortaharman } \\
\text { to Darikent }\end{array}$ & 1140 & 16.06.2014 & \multirow{2}{*}{ Steppe } & & \multirow{2}{*}{ Ir.-Tur. } \\
\hline & 4184 & $\begin{array}{l}\text { B7 Tunceli: Nazımiye, } 1 \mathrm{~km} \text { from Pülümür- } \\
\text { Nazımiye-Tunceli crossroads to Nazımiye }\end{array}$ & 1100 & 03.06 .2014 & & & \\
\hline Onobrychis subacaulis Boiss. & 4800 & $\begin{array}{l}\text { B8 Tunceli: Pülümür, } 2 \text { km from Hasangazi } \\
\text { to Kırklar }\end{array}$ & 1816 & 18.06.2014 & Steppe & Rcd. & Ir.-Tur. \\
\hline $\begin{array}{l}\text { Ononis spinosa L. subsp. } \\
\text { leiosperma (Boiss.) Sirj. }\end{array}$ & 5094 & $\begin{array}{l}\text { B7 Tunceli: Çemişgezek, } 2.5 \mathrm{~km} \text { from } \\
\text { Vişneli to Çemişgezek }\end{array}$ & 895 & 21.06 .2014 & $\begin{array}{l}\text { Wet } \\
\text { places, } \\
\text { steppe }\end{array}$ & & Unk. \\
\hline $\begin{array}{l}\text { Pisum sativum L. subsp. elatius } \\
\text { (M.Bieb.) Aschers. \& Graebn. } \\
\text { var. elatius }\end{array}$ & 6511 & $\begin{array}{l}\text { B7 Tunceli: Center, } 18 \mathrm{~km} \text { from Tunceli to } \\
\text { Ovacık (Munzur Valley) }\end{array}$ & 990 & 30.05 .2015 & \begin{tabular}{l|} 
Wet places \\
in Oak \\
forest
\end{tabular} & Rcd. & Medit. \\
\hline $\begin{array}{l}\text { Pisum sativum L. subsp. elatius } \\
\text { (M.Bieb.) Aschers. \& Graebn. } \\
\text { var. pumilio Meikle }\end{array}$ & 3869 & $\begin{array}{l}\text { B7 Tunceli: Pertek, } 5 \mathrm{~km} \text { from Pertek to } \\
\text { Çemişgezek }\end{array}$ & 866 & 24.05 .2014 & $\begin{array}{l}\text { Degraded } \\
\text { oak forest, } \\
\text { steppe } \\
\end{array}$ & & Unk. \\
\hline Sophora jaubertii Spach & 4432 & $\begin{array}{l}\text { B7 Tunceli: Pülümür, } 16 \text { km from Pülümür to } \\
\text { Tunceli (Pülümür valley) }\end{array}$ & 1240 & 05.06 .2014 & $\begin{array}{l}\text { Ruderal, } \\
\text { steppe }\end{array}$ & Red. & Euxine \\
\hline \multirow[b]{2}{*}{ Trifolium elongatum Willd. } & 4788 & B7 Tunceli: Pülümür, SE of Közlüce v. & 1620 & 18.06 .2014 & \multirow[b]{2}{*}{ Steppe } & \multirow[b]{2}{*}{$*$} & \multirow[b]{2}{*}{ Unk. } \\
\hline & 4794 & $\begin{array}{l}\text { B8 Tunceli: Pülümür, } 2 \mathrm{~km} \text { from Hasangazi } \\
\text { to Kırklar }\end{array}$ & 1816 & 18.06.2014 & & & \\
\hline Trifolium pilulare Boiss. & 4605 & $\begin{array}{l}\text { B7 Tunceli: Mazgirt, } 11 \mathrm{~km} \text { from Çukur to } \\
\text { Güleç }\end{array}$ & 1230 & 16.06.2014 & Steppe & & Unk. \\
\hline Trifolium resupinatum $\mathrm{L}$. & 4483 & $\begin{array}{l}\text { B7 Tunceli: Hozat, Çemişgezek-Elazı̆g road, } \\
\text { S of Akpınar v. }\end{array}$ & 1221 & 06.06 .2014 & Riverside & Red. & Unk. \\
\hline Vicia balansae Boiss. & 4288 & B7 Tunceli: Ovacık, Işıkvuran v. & 1710 & 04.06 .2014 & Steppe & Rcd. & Euxine \\
\hline $\begin{array}{l}\text { Vicia cracca } \text { L. subsp. } \\
\text { stenophylla } \text { Vel. }\end{array}$ & 4179 & $\begin{array}{l}\text { B7 Tunceli: Nazımiye, } 5 \mathrm{~km} \text { from Nazımiye } \\
\text { to Tunceli }\end{array}$ & 1435 & 03.06.2014 & Steppe & Red. & Unk. \\
\hline $\begin{array}{l}\text { Vicia noeana Boiss \& Reut. ex } \\
\text { Boiss. }\end{array}$ & 4167 & $\begin{array}{l}\text { B7 Tunceli: Nazımiye, } 5 \mathrm{~km} \text { from Nazımiye } \\
\text { to Tunceli }\end{array}$ & 1435 & 03.06.2014 & Steppe & & Ir.-Tur. \\
\hline \multirow{2}{*}{ Vicia peregrina $\mathrm{L}$. } & 4083 & $\begin{array}{l}\text { B7 Tunceli: Mazgirt, between Tunceli and } \\
\text { Kovancilar, around of Kuşcu }\end{array}$ & 867 & 02.06 .2014 & \multirow{2}{*}{$\begin{array}{l}\text { Steppe, } \\
\text { field side }\end{array}$} & \multirow{2}{*}{ Rcd. } & \multirow{2}{*}{ Unk. } \\
\hline & 4168 & $\begin{array}{l}\text { B7 Tunceli: Nazımiye, } 5 \mathrm{~km} \text { from Nazımiye } \\
\text { to Tunceli }\end{array}$ & 1435 & 03.06 .2014 & & & \\
\hline $\begin{array}{l}\text { Vicia sativa L. subsp. nigra (L.) } \\
\text { Ehrh. var. segetalis (Thuill.) Ser. } \\
\text { ex DC. }\end{array}$ & 3744 & B7 Tunceli: Center, $1 \mathrm{~km} \mathrm{~S}$ of Aktuluk & 930 & 22.05 .2014 & $\begin{array}{l}\text { Field side, } \\
\text { steppe }\end{array}$ & Red. & Unk. \\
\hline Vicia sepium $\mathrm{L}$. & 5066 & $\begin{array}{l}\text { B7 Tunceli: Çemişgezek, } 5 \mathrm{~km} \text { from } \\
\text { Çemişgezek to Pertek }\end{array}$ & 1050 & 21.06 .2014 & Steppe & Red. & $\begin{array}{l}\text { Euro.- } \\
\text { Sib. }\end{array}$ \\
\hline \multicolumn{8}{|l|}{ FAGACEAE Dumort. } \\
\hline \multirow[b]{2}{*}{ Quercus brantii Lindl. } & 5455 & B7 Tunceli: Hozat, S of Kurukaymak & 1495 & 21.07.2014 & \multirow[b]{2}{*}{ Forest } & & \multirow[b]{2}{*}{ Ir.-Tur. } \\
\hline & 6247 & $\begin{array}{l}\text { B7 Tunceli: Nazımiye, } 2 \mathrm{~km} \text { from Aşağırabat } \\
\text { bridge to Büyükyurt (Hakis) v. }\end{array}$ & 1095 & 05.09 .2014 & & & \\
\hline \multirow{5}{*}{$\begin{array}{l}\text { Quercus infectoria Oliv. subsp. } \\
\text { veneris (A.Kern.) Meikle }\end{array}$} & 5747 & B7 Tunceli: Mazgirt, SW of Güleç v. & 1105 & 05.08 .2014 & \multirow{5}{*}{ Forest } & & \multirow{5}{*}{ Unk. } \\
\hline & 5723 & B7 Tunceli: Mazgirt, NE of Akdüven v. & 1040 & 05.08 .2014 & & & \\
\hline & 6058 & B7 Tunceli: Center, $1 \mathrm{~km} \mathrm{~W}$ of Pınar v. & 1274 & 15.08 .2014 & & & \\
\hline & 6228 & $\begin{array}{l}\text { B7 Tunceli: Center, } 5 \mathrm{~km} \text { from Tunceli to } \\
\text { Pülümür (Pülümür Valley) }\end{array}$ & 930 & 05.09 .2014 & & & \\
\hline & 6248 & B7 Tunceli: Center, $1 \mathrm{~km}$ from Tunceli- & 1133 & 05.09 .2014 & & & \\
\hline
\end{tabular}




\begin{tabular}{|c|c|c|c|c|c|c|c|}
\hline & & Pülümür mainroad to Alacık v. & & & & & \\
\hline & 6119 & B7 Tunceli: Pertek, 2 km W of Bulgurtepe v. & 1060 & 17.08 .2014 & & & \\
\hline & 5710 & $\begin{array}{l}\text { B7 Tunceli: Pertek, between Koçpınar and } \\
\text { Biçmekaya }\end{array}$ & 1270 & 25.07.2014 & & & \\
\hline & 5711 & $\begin{array}{l}\text { B7 Tunceli: Pertek, between Koçpınar and } \\
\text { Çalıözü }\end{array}$ & 1130 & 25.07.2014 & & & \\
\hline \multirow{15}{*}{ Quercus libani Oliv. } & 5397 & $\begin{array}{l}\text { B7 Tunceli, Center, } 10 \mathrm{~km} \text { from Aktuluk to } \\
\text { Demirkap1 }\end{array}$ & 1450 & 21.07.2014 & \multirow{15}{*}{ Forest } & & \multirow{15}{*}{ Ir.-Tur. } \\
\hline & 5748 & B7 Tunceli: Mazgirt, SW of Güleç v. & 1105 & 05.08 .2014 & & & \\
\hline & 5731 & B7 Tunceli: Mazgirt, $\mathrm{N}$ of Akdüven v. & 1130 & 05.08 .2014 & & & \\
\hline & 5724 & B7 Tunceli: Mazgirt, NE of Akdüven v. & 1040 & 05.08 .2014 & & & \\
\hline & 6060 & B7 Tunceli: Center, 1 km W of Babaocağ1 v. & 1240 & 15.08 .2014 & & & \\
\hline & 6059 & B7 Tunceli: Center, Pınar v. & 1270 & 15.08 .2014 & & & \\
\hline & 5465 & B7 Tunceli: Center, W of Taşıtlı v. & 1490 & 21.07 .2014 & & & \\
\hline & 6249 & $\begin{array}{l}\text { B7 Tunceli: Center, } 1 \mathrm{~km} \text { from Tunceli- } \\
\text { Pülümür mainroad to Alackk v. }\end{array}$ & 1133 & 05.09 .2014 & & & \\
\hline & 6241 & $\begin{array}{l}\text { B7 Tunceli: Nazımiye, } 400 \mathrm{~m} \text { from } \\
\text { Aşağırabat bridge to Büyükyurt (Hakis) v. }\end{array}$ & 1070 & 05.09 .2014 & & & \\
\hline & 5315 & $\begin{array}{l}\text { B7 Tunceli: Nazımiye, } 5 \mathrm{~km} \text { from Nazımiye } \\
\text { to Tunceli }\end{array}$ & 1447 & 20.07 .2014 & & & \\
\hline & 5342 & B7 Tunceli: Nazımiye, E of Ramazanköy v. & 1440 & 20.07 .2014 & & & \\
\hline & 5905 & $\begin{array}{l}\text { B7 Tunceli: Ovacık, } 2.5 \mathrm{~km} \text { from Tunceli- } \\
\text { Ovacık-Yakatarla crossroads to Yakatarla }\end{array}$ & 1400 & 08.08 .2014 & & & \\
\hline & 5468 & $\begin{array}{l}\text { B7 Tunceli: Center, } 1.5 \mathrm{~km} \text { from Geyiksuyu } \\
\text { to Atadoğdu }\end{array}$ & 1225 & 21.07.2014 & & & \\
\hline & 6028 & B8 Tunceli: Pülümür, E of Altınhüseyin v. & 1633 & 14.08 .2014 & & & \\
\hline & 6152 & $\begin{array}{l}\text { B7 Tunceli: Pülümür, } 3 \text { km from Erzincan- } \\
\text { Pülümür-Süleymanuşağ1 to Süleymanyuşağ1 }\end{array}$ & 1800 & 27.08 .2014 & & & \\
\hline $\begin{array}{l}\text { Quercus macranthera Fisch. \& } \\
\text { C.A.Mey. subsp. syspirensis } \\
\text { (K.Koch) Mentsky }\end{array}$ & 6189 & B7 Tunceli: Center, E of Çıralı v. & 1450 & 27.08 .2014 & Forest & $*$ & Unk. \\
\hline $\begin{array}{l}\text { Quercus petraea (Matt.) Liebl. } \\
\text { subsp. iberica (Steven ex } \\
\text { M.Bieb). Krassiln. }\end{array}$ & 5396 & $\begin{array}{l}\text { B7 Tunceli, Center, } 10 \mathrm{~km} \text { from Aktuluk to } \\
\text { Demirkap1 }\end{array}$ & 1450 & 21.07.2014 & Forest & & Unk. \\
\hline \multirow{7}{*}{$\begin{array}{l}\text { Quercus petraea (Matt.) Liebl. } \\
\text { subsp. pinnatiloba (K. Koch) } \\
\text { Menitsky }\end{array}$} & 5461 & $\begin{array}{l}\text { B7 Tunceli: Hozat, between Beşelma and } \\
\text { Uzundal }\end{array}$ & 1787 & 21.07.2014 & \multirow{7}{*}{ Forest } & \multirow{7}{*}{$*$} & \multirow{7}{*}{ Unk. } \\
\hline & 5868 & $\begin{array}{l}\text { B7 Tunceli: Nazımiye, } 5 \mathrm{~km} \mathrm{NE} \text { of } \\
\text { Büyükyurt (Hakis) v. }\end{array}$ & 1835 & 07.08 .2014 & & & \\
\hline & 5343 & B7 Tunceli: Nazımiye, E of Ramazanköy v. & 1440 & 20.07 .2014 & & & \\
\hline & 5904 & $\begin{array}{l}\text { B7 Tunceli: Ovacık, } 2.5 \mathrm{~km} \text { from Tunceli- } \\
\text { Ovacık-Yakatarla crossroads to Yakatarla }\end{array}$ & 1400 & 08.08 .2014 & & & \\
\hline & 6252 & B7 Tunceli: Center, Alacık road & 1330 & 05.09 .2014 & & & \\
\hline & 5527 & $\begin{array}{l}\text { B8 Tunceli: Pülümür, } 3.5 \mathrm{~km} \text { from Bardakçı } \\
\text { to Derindere }\end{array}$ & 1746 & 22.07.2014 & & & \\
\hline & 5560 & $\begin{array}{l}\text { B8 Tunceli: Pülümür, between Pülümür and } \\
\text { Yedisu, } 2.5 \mathrm{~km} \text { after Derindere v. }\end{array}$ & 1925 & 22.07 .2014 & & & \\
\hline \multirow[b]{2}{*}{ Quercus pubescens Willd. } & 5459 & B7 Tunceli: Hozat, S of Kurukaymak & 1405 & 21.07 .2014 & \multirow[b]{2}{*}{ Forest } & \multirow[b]{2}{*}{ Red. } & \multirow[b]{2}{*}{ Unk. } \\
\hline & 6150 & $\begin{array}{l}\text { B7 Tunceli: Pülümür, } 2 \text { km from Erzincan- } \\
\text { Pülümür-Süleymanuşağı to Süleymanyuşağ1 }\end{array}$ & 1683 & 27.08 .2014 & & & \\
\hline \multirow{6}{*}{$\begin{array}{l}\text { Quercus robur L. subsp. } \\
\text { pedunculiflora (K.Koch) } \\
\text { Menitsky }\end{array}$} & 6192 & $\begin{array}{l}\text { B7 Tunceli: Center, } 3 \mathrm{~km} \text { from Gökçek to } \\
\text { Çıralı }\end{array}$ & 1400 & 27.08 .2014 & \multirow{6}{*}{ Forest } & & \multirow{6}{*}{ Ir.-Tur. } \\
\hline & 6212 & $\begin{array}{l}\text { B7 Tunceli: Ovacık, Mollaaliler v. (Mercan } \\
\text { Valley) }\end{array}$ & 1394 & 28.08 .2014 & & & \\
\hline & 5899 & $\begin{array}{l}\text { B7 Tunceli: Ovackk, Tunceli-Ovacık- } \\
\text { Yakatarla junction }\end{array}$ & 1240 & 08.08.2014 & & & \\
\hline & 6250 & B7 Tunceli: Center, Alacık road & 1330 & 05.09 .2014 & & & \\
\hline & 6187 & $\begin{array}{l}\text { B7 Tunceli: Center, } 15 \mathrm{~km} \text { from Tunceli- } \\
\text { Pülümür-Sütlüce crossroads to Çıralı v. (10 } \\
\text { km before Çıralı v.) }\end{array}$ & 1791 & 27.08 .2014 & & & \\
\hline & 6034 & $\begin{array}{l}\text { B8 Tunceli: Pülümür, } 2 \mathrm{~km} \mathrm{~W} \text { of } \\
\text { Altınhüseyin }\end{array}$ & 1808 & 14.08 .2014 & & & \\
\hline \multicolumn{8}{|l|}{ GENTIANACEAE Juss. } \\
\hline \multirow{4}{*}{$\begin{array}{l}\text { Centaurium erythraea Rafn. } \\
\text { subsp. turcicum (Velen.) } \\
\text { Melderis }\end{array}$} & 5445 & $\begin{array}{l}\text { B7 Tunceli: Hozat, N of Boydaş v. (Tahar } \\
\text { Valley) }\end{array}$ & 1215 & 21.07.2014 & \multirow{4}{*}{$\begin{array}{l}\text { Steppe, } \\
\text { wet places }\end{array}$} & & \multirow{4}{*}{ Unk. } \\
\hline & 5615 & B7 Tunceli: Nazımiye, 1 km S of Holik v. & 1875 & 23.07 .2014 & & & \\
\hline & 5913 & $\begin{array}{l}\text { B7 Tunceli: Ovacık, } 3 \text { km N of Işıkvuran v. } \\
\text { Munzur Mt. }\end{array}$ & 1800 & 08.08.2014 & & & \\
\hline & 5520 & $\begin{array}{l}\text { B8 Tunceli: Pülümür, } 300 \text { m from Bardakçı- } \\
\text { Derindere-Kırdım crossroads to Kırdım v. }\end{array}$ & 1750 & 22.07 .2014 & & & \\
\hline Gentiana gelida M.Bieb. & 5789 & $\begin{array}{l}\text { B7 Tunceli: Pülümür, between Yalmanlar } \\
\text { and Kocatepe, NW of Sarıül v. }\end{array}$ & 2230 & 06.08 .2014 & $\begin{array}{l}\text { Mt. } \\
\text { pastures }\end{array}$ & Rcd. & $\begin{array}{l}\text { Euxine } \\
\text { (Mt.) }\end{array}$ \\
\hline Gentiana olivieri Griseb. & 4158 & $\begin{array}{l}\text { B7 Tunceli: Nazımiye, } 6.5 \mathrm{~km} \text { from } \\
\text { Nazımiye to Tunceli, } 400 \mathrm{~m} \text { after Geriş v. } \\
\text { junction }\end{array}$ & 1340 & 03.06 .2014 & $\begin{array}{l}\text { Damp } \\
\text { places }\end{array}$ & & Ir.-Tur. \\
\hline
\end{tabular}




\begin{tabular}{|c|c|c|c|c|c|c|c|}
\hline & 4975 & $\begin{array}{l}\text { B7 Tunceli: Center, } 2 \mathrm{~km} \text { from Sarıtaş to } \\
\text { Gözen (İksor), Kalan Valley }\end{array}$ & 1180 & 19.06.2014 & & & \\
\hline Gentiana septemfida Pallas & 5935 & $\begin{array}{l}\text { B7 Tunceli: Ovacık, } 7 \mathrm{~km} \text { from Işıkvuran to } \\
\text { Eskigedik }\end{array}$ & 2225 & 08.08 .2014 & $\begin{array}{l}\text { Damp } \\
\text { places }\end{array}$ & Rcd. & $\begin{array}{l}\text { Hyrc.- } \\
\text { Euxine } \\
\text { (Mt.) }\end{array}$ \\
\hline \multicolumn{8}{|l|}{ GERANIACEAE Juss. } \\
\hline Erodium ciconium (L.) L'Hér. & 6434 & B7 Tunceli: Center, Alibaba nh. & 930 & 11.04 .2015 & Steppe & & Unk. \\
\hline \multirow[b]{2}{*}{ Erodium cicutarium (L.) L Hér. } & 4522 & B7 Tunceli: Mazgirt, $1 \mathrm{~km} \mathrm{~W}$ of Kayacı v. & 990 & 16.06 .2014 & \multirow[b]{2}{*}{ Steppe } & \multirow[b]{2}{*}{ Rcd. } & \multirow[b]{2}{*}{ Cosmp. } \\
\hline & 6366 & $\begin{array}{l}\text { B7 Tunceli: Center, } 9 \mathrm{~km} \text { from Tunceli to } \\
\text { Pülümür (Pülümür Valley) }\end{array}$ & 921 & 10.02 .2015 & & & \\
\hline \multirow{3}{*}{ Geranium pyrenaicum Burm.f. } & 4951 & $\begin{array}{l}\text { B7 Tunceli: Ovacık, } \mathrm{N} \text { of Eskigedik v., } \\
\text { Munzur Mt. }\end{array}$ & 2160 & 19.06.2014 & \multirow{3}{*}{$\begin{array}{l}\text { Rocky } \\
\text { areas, } \\
\text { steppe }\end{array}$} & & \multirow{3}{*}{ Unk. } \\
\hline & 4375 & B7 Tunceli: Pülümür, Kocatepe v. & 1680 & 05.06 .2014 & & & \\
\hline & 4862 & $\begin{array}{l}\text { B8 Tunceli: Pülümür, } 4 \text { km from Kırklar to } \\
\text { Kızılmescit }\end{array}$ & 2450 & 18.06 .2014 & & & \\
\hline \multirow{5}{*}{ Geranium rotundifolium $\mathrm{L}$. } & 4963 & $\begin{array}{l}\text { B7 Tunceli: Ovacık, } \mathrm{N} \text { of Eskigedik v., } \\
\text { Munzur Mt. }\end{array}$ & 2160 & 19.06.2014 & \multirow{5}{*}{$\begin{array}{l}\text { Rocky } \\
\text { areas, } \\
\text { steppe }\end{array}$} & & \multirow{5}{*}{ Unk. } \\
\hline & 4286 & $\begin{array}{l}\text { B7 Tunceli: Ovacık, } 3 \text { km N of Işıkvuran v. } \\
\text { Munzur Mt. }\end{array}$ & 1960 & 04.06 .2014 & & & \\
\hline & 4238 & $\begin{array}{l}\text { B7 Tunceli: Ovacık, } 18 \mathrm{~km} \text { from Ovacık- } \\
\text { Tunceli-Ișikvuran crossroads to Ișıkvuran }\end{array}$ & 1635 & 04.06 .2014 & & & \\
\hline & 6700 & $\begin{array}{l}\text { B7 Tunceli: Center, } 3 \mathrm{~km} \text { from Tunceli to } \\
\text { Ovack (Munzur Valley) }\end{array}$ & 915 & 16.04 .2015 & & & \\
\hline & 6502 & $\begin{array}{l}\text { B7 Tunceli: Center, } 1 \mathrm{~km} \text { from Sarıtaş to } \\
\text { Gözen (İksor), Kalan Valley }\end{array}$ & 1160 & 30.05 .2015 & & & \\
\hline \multirow{2}{*}{ Geranium tuberosum $\mathrm{L}$. } & 4660 & $\begin{array}{l}\text { B7 Tunceli: Hozat, between Karacaköy and } \\
\text { Uzundal, around of Sarısaltuk Türbesi }\end{array}$ & 2270 & 17.06 .2014 & \multirow{2}{*}{ Steppe } & & \multirow{2}{*}{ Ir.-Tur. } \\
\hline & 4261 & $\begin{array}{l}\text { B7 Tunceli: Ovacık, } 3 \text { km N of Işıkvuran v. } \\
\text { Munzur Mt. }\end{array}$ & 2100 & 04.06 .2014 & & & \\
\hline \multirow{2}{*}{$\begin{array}{l}\text { Pelargonium endlicherianum } \\
\text { Fenzl }\end{array}$} & 5199 & $\begin{array}{l}\text { B7 Tunceli: Çemişgezek, } 2 \mathrm{~km} \text { from } \\
\text { Dedebeyli to Akçayurt }\end{array}$ & 1100 & 29.06 .2014 & \multirow{2}{*}{ Scree } & & \multirow{2}{*}{ Unk. } \\
\hline & 5658 & $\begin{array}{l}\text { B7 Tunceli: Ovacık, } 4 \text { km from Eğripınar to } \\
\text { Yenikonak }\end{array}$ & 1455 & 24.07 .2014 & & & \\
\hline \multicolumn{8}{|l|}{ HYDROCHARITACEAE JusS. } \\
\hline \multirow{2}{*}{$\begin{array}{l}\text { Najas marina L. subsp. } \\
\text { intermedia (Wolfg. ex Gorski) } \\
\text { Casper }\end{array}$} & 5958 & $\begin{array}{l}\text { B7 Tunceli: Pertek, } 9 \mathrm{~km} \text { from Pertek to } \\
\text { Çemişgezek, } 2.5 \mathrm{~km} \text { before Singeç bridge }\end{array}$ & 875 & 13.08 .2014 & \multirow{2}{*}{$\begin{array}{l}\text { In the lake } \\
\text { water }\end{array}$} & \multirow{2}{*}{ Rcd. } & \multirow{2}{*}{ Unk. } \\
\hline & 5959 & $\begin{array}{l}\text { B7 Tunceli: Pertek, } 9 \mathrm{~km} \text { from Pertek to } \\
\text { Çemişgezek, } 2.5 \mathrm{~km} \text { before Singeç bridge }\end{array}$ & 875 & 13.08 .2014 & & & \\
\hline \multicolumn{8}{|l|}{ JUGLANDACEAE DC. ex Perleb } \\
\hline Juglans regia $\mathrm{L}$. & 6331 & $\begin{array}{l}\text { B7 Tunceli: Center, } 17 \mathrm{~km} \text { from Tunceli to } \\
\text { Ovacık (Munzur Valley) }\end{array}$ & 1014 & 21.09 .2014 & $\begin{array}{l}\text { Riverside, } \\
\text { in gardens }\end{array}$ & & Unk. \\
\hline \multicolumn{8}{|l|}{ JUNCACEAE Juss. } \\
\hline Juncus alpigenus K.Koch. & 4930 & $\begin{array}{l}\text { B7 Tunceli: Ovacık, } 8 \text { km from Eskigedik to } \\
\text { Ișıkvuran }\end{array}$ & 2120 & 19.06 .2014 & $\begin{array}{l}\text { Damp } \\
\text { places }\end{array}$ & Rcd. & Unk. \\
\hline Juncus articulatus $\mathrm{L}$. & 5513 & $\begin{array}{l}\text { B8 Tunceli: Pülümür, between Bardakçı to } \\
\text { Derindere }\end{array}$ & 1980 & 22.07 .2014 & $\begin{array}{l}\text { Damp } \\
\text { places }\end{array}$ & & Unk. \\
\hline \multicolumn{8}{|l|}{ JUNCAGINACEAE Juss. } \\
\hline Triglochin palustris $\mathrm{L}$. & 5519 & $\begin{array}{l}\text { B8 Tunceli: Pülümür, between Bardakçı to } \\
\text { Derindere }\end{array}$ & 1980 & 22.07 .2014 & $\begin{array}{l}\text { Wet } \\
\text { meadows }\end{array}$ & Rcd. & Unk. \\
\hline LAMIACEAE Martinov & & & & & & & \\
\hline Ajuga chamaepitys (L.) Schreb. & 4478 & B7 Tunceli: Hozat, $2 \mathrm{~km} \mathrm{NE}$ of Akpınar v. & 1350 & \begin{tabular}{|l|}
06.06 .2014 \\
\end{tabular} & Stenn & & Int \\
\hline subsp. chia (Schreb.) Arcang. & 5655 & B7 Tunceli: Ovacık, SW of Gözeler v. & 1420 & 24.07 .2014 & Steppe & & Unk. \\
\hline $\begin{array}{l}\text { Ajuga chamaepitys (L.) Schreb. } \\
\text { subsp. euphratica } \text { P.H.Davis }\end{array}$ & 5164 & $\begin{array}{l}\text { B7 Tunceli: Çemişgezek, Tahar valley (Ali } \\
\text { Boğazı) }\end{array}$ & 1024 & 29.06 .2014 & Steppe & \begin{tabular}{|l|}
$*$ \\
Rcd.
\end{tabular} & Ir.-Tur. \\
\hline & 4301 & $\begin{array}{l}\text { B7 Tunceli: Ovacık, } 13 \text { km from Işıkvuran to } \\
\text { Ağaçpınar }\end{array}$ & 1875 & 04.06 .2014 & & & \\
\hline Ajuga chamaepitys (L.) Schreb. & 5046 & $\begin{array}{l}\text { B7 Tunceli: Ovacık, between Yazı̈ren and } \\
\text { Gözeler }\end{array}$ & 1400 & 20.06 .2014 & & & \\
\hline $\begin{array}{l}\text { subsp. laevigata (Banks \& Sol.) } \\
\text { P.H.Davis }\end{array}$ & 6489 & $\begin{array}{l}\text { B7 Tunceli: Pülümür, } 1 \mathrm{~km} \text { from Turnadere } \\
\text { to Közlüce }\end{array}$ & 1435 & 29.05 .2015 & Steppe & & Ir.-Tur. \\
\hline & 6374 & $\begin{array}{l}\text { B7 Tunceli: Nazımiye, } 31 \mathrm{~km} \text { from Tunceli } \\
\text { to Pülümür (Pülümür Valley) }\end{array}$ & 1048 & 11.03 .2015 & & & \\
\hline $\begin{array}{l}\text { Ballota nigra L. subsp. kurdica } \\
\text { P.H.Davis }\end{array}$ & 5621 & B7 Tunceli: Nazımiye, 1 km S of Holik v. & 1990 & 23.07 .2014 & $\begin{array}{l}\text { Rock } \\
\text { crevices }\end{array}$ & Rcd. & Ir.-Tur. \\
\hline $\begin{array}{l}\text { Clinopodium graveolens } \\
\text { (M.Bieb.) Kuntze subsp. }\end{array}$ & 4961 & $\begin{array}{l}\text { B7 Tunceli: Ovacık, N of Eskigedik v., } \\
\text { Munzur Mt. }\end{array}$ & 2160 & 19.06.2014 & & & \\
\hline $\begin{array}{l}\text { graveolens (It is mantioned as } \\
\text { Acinos rotundifolius Pers. in flora } \\
\text { of Turkey) }\end{array}$ & 4776 & $\begin{array}{l}\text { B7 Tunceli: Pülümür, } 2 \text { km from Turnadere } \\
\text { to Közlüce }\end{array}$ & 1560 & 18.06 .2014 & Steppe & Red. & Unk. \\
\hline Clinopodium vulgare $\mathrm{L}$. & 5257 & $\begin{array}{l}\text { B7 Tunceli: Nazımiye, Pülümür Valley, E } \\
\text { slopes of Hılbeşen bridge, across of Ağlayan } \\
\text { Kayalar }\end{array}$ & 1560 & 30.06 .2014 & $\begin{array}{l}\text { Rock } \\
\text { crevices }\end{array}$ & Rcd. & Unk. \\
\hline
\end{tabular}




\begin{tabular}{|c|c|c|c|c|c|c|c|}
\hline \multirow{4}{*}{$\begin{array}{l}\text { Lallemantia iberica (M.Bieb.) } \\
\text { Fisch. \& C.A.Mey. }\end{array}$} & 3736 & B7 Tunceli: Center, $1 \mathrm{~km} \mathrm{~S}$ of Aktuluk & 930 & 22.05 .2014 & \multirow{4}{*}{ Steppe } & & \multirow{4}{*}{ Ir.-Tur. } \\
\hline & 3861 & $\begin{array}{l}\text { B7 Tunceli: Center, between Tunceli and } \\
\text { Pertek, junction of Tozkoparan v. }\end{array}$ & 1280 & 24.05 .2014 & & & \\
\hline & 4064 & $\begin{array}{l}\text { B7 Tunceli: Ovacık, } 51 \mathrm{~km} \text { from Tunceli to } \\
\text { Ovacık (Munzur Valley), } 7 \mathrm{~km} \text { to Ovacık }\end{array}$ & 1210 & 26.05 .2014 & & & \\
\hline & 4425 & $\begin{array}{l}\text { B7 Tunceli: Pülümür, } 9 \mathrm{~km} \text { from Pülümür to } \\
\text { Tunceli (Pülümür valley), S of Kangallı v. }\end{array}$ & 1324 & 05.06 .2014 & & & \\
\hline \multirow{3}{*}{$\begin{array}{l}\text { Lallemantia peltata (L.) Fisch. \& } \\
\text { C.A.Mey. }\end{array}$} & 4960 & $\begin{array}{l}\text { B7 Tunceli: Ovacık, N of Eskigedik v., } \\
\text { Munzur Mt. }\end{array}$ & 2160 & 19.06.2014 & \multirow{3}{*}{ Steppe } & & \multirow{3}{*}{ Ir.-Tur. } \\
\hline & 4398 & $\begin{array}{l}\text { B7 Tunceli: Pülümür, } 5 \mathrm{~km} \text { from Çakırkaya } \\
\text { and Dereboyu }\end{array}$ & 1870 & 05.06 .2014 & & & \\
\hline & 4348 & $\begin{array}{l}\text { B7 Tunceli: Pülümür, } 2.5 \mathrm{~km} \text { from Kocatepe } \\
\text { to Sarıgül, near to Sarıül junction }\end{array}$ & 1970 & 05.06 .2014 & & & \\
\hline \multirow{2}{*}{$\begin{array}{l}\text { Lamium album L. subsp. crinitum } \\
\text { (Montbret \& Aucher ex Benth.) } \\
\text { Mennema }\end{array}$} & 4671 & $\begin{array}{l}\text { B7 Tunceli: Hozat, between Karacaköy and } \\
\text { Uzundal, around of Sarısaltuk Türbesi }\end{array}$ & 2270 & 17.06 .2014 & \multirow{2}{*}{ Steppe } & & \multirow{2}{*}{ Euxine } \\
\hline & 6537 & $\begin{array}{l}\text { B7 Tunceli: Ovacık, } 7 \mathrm{~km} \text { from Yoncalı to } \\
\text { Ağaçpınar }\end{array}$ & 2015 & 01.06 .2015 & & & \\
\hline \multirow{2}{*}{ Lamium amplexicaule L. } & 6431 & $\begin{array}{l}\text { B7 Tunceli: Center, } 7 \mathrm{~km} \text { from Tunceli to } \\
\text { Pülümür (Pülümür Valley) }\end{array}$ & 934 & 12.04 .2015 & \multirow{2}{*}{ Steppe } & & \multirow{2}{*}{ Unk. } \\
\hline & 6375 & $\begin{array}{l}\text { B7 Tunceli: Center, } 14 \mathrm{~km} \text { from Tunceli to } \\
\text { Ovacık (Munzur Valley) }\end{array}$ & 1000 & 11.04 .2015 & & & \\
\hline \multirow{6}{*}{$\begin{array}{l}\text { Lamium garganicum L. subsp. } \\
\text { striatum (Sm.) Hayek }\end{array}$} & 4672 & $\begin{array}{l}\text { B7 Tunceli: Hozat, between Karacaköy and } \\
\text { Uzundal, around of Sarısaltuk Türbesi }\end{array}$ & 2270 & 17.06 .2014 & \multirow{6}{*}{$\begin{array}{l}\text { In shadow } \\
\text { of rocks, } \\
\text { rocky } \\
\text { areas }\end{array}$} & & \multirow{6}{*}{ Unk. } \\
\hline & 6477 & $\begin{array}{l}\text { B7 Tunceli: Nazımiye, Pülümür Valley, } 43 \\
\text { km from Tunceli to Pülümür }\end{array}$ & 1208 & 29.05 .2015 & & & \\
\hline & 4248 & $\begin{array}{l}\text { B7 Tunceli: Ovacık, } 3 \text { km N of Işıkvuran v. } \\
\text { Munzur Mt. }\end{array}$ & 2020 & 04.06 .2014 & & & \\
\hline & 6533 & $\begin{array}{l}\text { B7 Tunceli: Ovacık, } 7 \mathrm{~km} \text { from Yoncalı to } \\
\text { Ağaçpınar }\end{array}$ & 2165 & 01.06 .2015 & & & \\
\hline & 4766 & $\begin{array}{l}\text { B7 Tunceli: Pülümür, } 2 \mathrm{~km} \text { from Turnadere } \\
\text { to Közlüce }\end{array}$ & 1575 & 18.06 .2014 & & & \\
\hline & 6488 & $\begin{array}{l}\text { B7 Tunceli: Pülümür, } 2 \mathrm{~km} \text { from Turnadere } \\
\text { to Közlüce }\end{array}$ & 1528 & 29.05 .2015 & & & \\
\hline Lamium macrodon Boiss. \& Huet & 6376 & $\begin{array}{l}\text { B7 Tunceli: Center, S of Pah bridge, } \\
\text { Karakoç-Atlant junction }\end{array}$ & 939 & 06.03 .2015 & Steppe & & Ir.-Tur. \\
\hline Lamium multifidum L. & 6480 & $\begin{array}{l}\text { B7 Tunceli: Pülümür, } 45 \mathrm{~km} \text { from Tunceli to } \\
\text { Pülümür (Pülümür Valley) }\end{array}$ & 1194 & 29.05 .2015 & Steppe & Rcd. & Ir.-Tur. \\
\hline Lamium tomentosum Willd. & 5817 & $\begin{array}{l}\text { B7 Tunceli: Ovacık, } 8 \mathrm{~km} \text { from Sarıül to } \\
\text { Yalmanlar, the walk way of Buyer Baba } \\
\text { sanctuary }\end{array}$ & 2680 & 06.08 .2014 & $\begin{array}{l}\text { Rocky } \\
\text { areas }\end{array}$ & Rcd. & Unk. \\
\hline Lycopus europaeus L. & 6137 & B7 Tunceli: Mazgirt, S of Dedebağ v. & 860 & 26.08 .2014 & Riverside & Rcd. & Unk. \\
\hline \multirow{2}{*}{ Marrubium anisodon K.Koch } & 4093 & $\begin{array}{l}\text { B7 Tunceli: Mazgirt, between Tunceli and } \\
\text { Kovancilar, around of Kuşcu }\end{array}$ & 867 & 02.06 .2014 & \multirow{2}{*}{ Steppe } & \multirow{2}{*}{ Rcd. } & \multirow{2}{*}{ Unk. } \\
\hline & 3795 & $\begin{array}{l}\text { B7 Tunceli: Center, between Tunceli and } \\
\text { Sütlüce, } 1.8 \mathrm{~km} \text { from Sütlüce junction }\end{array}$ & 1030 & 23.05 .2014 & & & \\
\hline \multirow{2}{*}{ Marrubium astracanicum Jacq. } & 4669 & $\begin{array}{l}\text { B7 Tunceli: Hozat, between Karacaköy and } \\
\text { Uzundal, around of Sarısaltuk Türbesi }\end{array}$ & 2270 & 17.06 .2014 & \multirow{2}{*}{ Steppe } & & \multirow{2}{*}{ Unk. } \\
\hline & 4856 & $\begin{array}{l}\text { B8 Tunceli: Pülümür, } 4 \text { km from Kırklar to } \\
\text { Kızılmescit }\end{array}$ & 2450 & 18.06 .2014 & & & \\
\hline $\begin{array}{l}\text { Marrubium parviflorum Fisch. \& } \\
\text { C.A.Mey. subsp. oligodon } \\
\text { (Boiss.) Seybold }\end{array}$ & 5285 & $\begin{array}{l}\text { B7 Tunceli: Pertek, } 8 \mathrm{~km} \text { from Pertek to } \\
\text { Tunceli }\end{array}$ & 1300 & 19.07.2014 & Steppe & Red. & Ir.-Tur. \\
\hline \multirow{2}{*}{ Melissa officinalis L. } & 5158 & $\begin{array}{l}\text { B7 Tunceli: Çemişgezek, between Anıl and } \\
\text { Gözlüçayır villages (Tahar Valley) }\end{array}$ & 1000 & 29.06 .2014 & \multirow{2}{*}{ Steppe } & & \multirow{2}{*}{ Euxine } \\
\hline & 5676 & $\begin{array}{l}\text { B7 Tunceli: Center, } 26 \mathrm{~km} \text { from Tunceli to } \\
\text { Ovacık (Munzur Valley) }\end{array}$ & 1075 & 24.07 .2014 & & & \\
\hline $\begin{array}{l}\text { Mentha longifolia (L.) L. subsp. } \\
\text { typhoides (Briq.) Harley }\end{array}$ & 5998 & $\begin{array}{l}\text { B7 Tunceli: Pülümür, } 1.5 \mathrm{~km} \text { from Turnadere } \\
\text { to Közlüce }\end{array}$ & 1500 & 14.08 .2014 & Riverside & & Unk. \\
\hline $\begin{array}{l}\text { Micromeria cremnophila } \text { Boiss. } \\
\text { \& Heldr. subsp. anatolica } \mathrm{P} . \mathrm{H} . \\
\text { Davis }\end{array}$ & 5165 & $\begin{array}{l}\text { B7 Tunceli: Çemişgezek, Tahar valley (Ali } \\
\text { Boğazı) }\end{array}$ & 1024 & 29.06 .2014 & $\begin{array}{l}\text { Rock } \\
\text { crevices }\end{array}$ & $*$ & Ir.-Tur. \\
\hline \multirow{2}{*}{$\begin{array}{l}\text { Micromeria cristata (Hampe) } \\
\text { Griseb. subsp. orientalis } \text { P.H. } \\
\text { Davis }\end{array}$} & 5241 & $\begin{array}{l}\text { B7 Tunceli: Nazımiye, } 800 \mathrm{~m} \text { from } \\
\text { Aşağırabat bridge to Büyükyurt (Hakis) v. }\end{array}$ & 1070 & 30.06 .2014 & Rock & $*$ & \\
\hline & 5932 & $\begin{array}{l}\text { B7 Tunceli: Ovacık, } 3 \text { km N of Işıkvuran v. } \\
\text { Munzur Mt. }\end{array}$ & 2184 & 08.08 .2014 & crevices & & Ir.-Tur. \\
\hline & 4566 & $\begin{array}{l}\text { B7 Tunceli: Mazgirt, } 4 \mathrm{~km} \text { from Ortaharman } \\
\text { to Darkent }\end{array}$ & 1140 & 16.06 .2014 & & & \\
\hline Moluccella laevis L. & 4070 & $\begin{array}{l}\text { B7 Tunceli: Mazgirt, between Tunceli and } \\
\text { Kovancilar, around of Kuşcu }\end{array}$ & 867 & 02.06 .2014 & $\begin{array}{l}\text { Field side, } \\
\text { steppe }\end{array}$ & Rcd. & Ir.-Tur. \\
\hline & 6262 & $\begin{array}{l}\text { B7 Tunceli: Mazgirt, between Kepektaşı and } \\
\text { Kuşcu }\end{array}$ & 875 & 19.09 .2014 & & & \\
\hline Nepeta betonicifolia C.A.Mey. & 4811 & B8 Tunceli: Pülümür, 2 km E of Kırklar v. & 2100 & 18.06 .2014 & Steppe & & Ir.-Tur. \\
\hline Nepeta italica $\mathrm{L}$. & 4150 & $\begin{array}{l}\text { B7 Tunceli: Mazgirt, } 2 \mathrm{~km} \text { from } \\
\text { Beylermezrası to Aslanyurdu }\end{array}$ & 855 & 02.06 .2014 & Steppe & & Medit. \\
\hline & 4992 & B7 Tunceli: Ovacık, W of Ziyaret v. & 1340 & 20.06 .2014 & & & \\
\hline
\end{tabular}




\begin{tabular}{|c|c|c|c|c|c|c|c|}
\hline & 3933 & $\begin{array}{l}\text { B7 Tunceli: Center, } 2.5 \mathrm{~km} \text { from Tunceli to } \\
\text { Ovacık (Munzur Valley) }\end{array}$ & 930 & 25.05 .2014 & & & \\
\hline $\begin{array}{l}\text { Nepeta leptantha Boiss. \& } \\
\text { Hausskn. }\end{array}$ & 5833 & $\begin{array}{l}\text { B7 Tunceli: Ovacik, } 800 \mathrm{~m} \mathrm{NE} \text { of Buyer } \\
\text { Baba Lake }\end{array}$ & 2910 & 06.08 .2014 & Mt. steppe & $*$ & Ir.-Tur. \\
\hline \multirow{2}{*}{$\begin{array}{l}\text { Nepeta nuda L. subsp. albiflora } \\
\text { (Boiss.) Gams }\end{array}$} & 4748 & B7 Tunceli: Ovacık, $3 \mathrm{~km} \mathrm{~S}$ of Ovacık & 1315 & 17.06 .2014 & \multirow{2}{*}{ Steppe } & & \multirow{2}{*}{ Unk. } \\
\hline & 6088 & B8 Tunceli: Pülümür, 2.3 km E of Kırklar v. & 2110 & 16.08 .2014 & & & \\
\hline \multirow{5}{*}{ Nepeta teucriifolia Willd. } & 5901 & $\begin{array}{l}\text { B7 Tunceli: Ovacik, Tunceli-Ovacık- } \\
\text { Yakatarla junction }\end{array}$ & 1240 & 08.08 .2014 & \multirow{5}{*}{ Steppe } & & \multirow{5}{*}{ Ir.-Tur. } \\
\hline & 5581 & B7 Tunceli: Pülümür, 2 km N of Dağyolu v. & 1566 & 22.07 .2014 & & & \\
\hline & 5381 & $\begin{array}{l}\text { B8 Tunceli: Nazımiye, } 6 \mathrm{~km} \text { from Sarıyayla } \\
\text { to Çalıkağıl }\end{array}$ & 2010 & 20.07 .2014 & & & \\
\hline & 6032 & B8 Tunceli: Pülümür, SE of Altınhüseyin & 1630 & 14.08 .2014 & & & \\
\hline & 5550 & $\begin{array}{l}\text { B8 Tunceli: Pülümür, between Pülümür and } \\
\text { Yedisu, } 9 \mathrm{~km} \text { after Derindere v. }\end{array}$ & 1780 & 22.07 .2014 & & & \\
\hline $\begin{array}{l}\text { Nepeta trichocalyx Greuter \& } \\
\text { Burdet }\end{array}$ & 5988 & $\begin{array}{l}\text { B7 Tunceli: Nazımiye, } 10 \mathrm{~km} \mathrm{NE} \mathrm{of} \\
\text { Büyükyurt (Hakis) v. }\end{array}$ & 1940 & 14.08 .2014 & Steppe & $*$ & Ir.-Tur. \\
\hline \multirow{3}{*}{$\begin{array}{l}\text { Origanum acutidens (Hand.- } \\
\text { Mazz.) Ietsw. }\end{array}$} & 3990 & $\begin{array}{l}\text { B7 Tunceli: Center, between Tunceli and } \\
\text { Ovacık (Munzur Valley), road of Dilek v. }\end{array}$ & 1043 & 25.05 .2014 & \multirow{3}{*}{ Steppe } & \multirow{3}{*}{ * } & \multirow{3}{*}{ Ir.-Tur. } \\
\hline & 5309 & $\begin{array}{l}\text { B7 Tunceli: Nazımiye, } 3 \mathrm{~km} \text { from Tunceli- } \\
\text { Pülümür-Nazımiye crossroads to Nazımiye }\end{array}$ & 1190 & 20.07.2014 & & & \\
\hline & 5762 & $\begin{array}{l}\text { B7 Tunceli: Pülümür, } 45 \mathrm{~km} \text { from Tunceli to } \\
\text { Pülümür (Pülümür valley) }\end{array}$ & 1200 & 06.08 .2014 & & & \\
\hline $\begin{array}{l}\text { Origanum vulgare L. subsp. } \\
\text { gracile (K.Koch) Ietsw. }\end{array}$ & 5163 & $\begin{array}{l}\text { B7 Tunceli: Çemişgezek, between Anıl and } \\
\text { Gözlüçayır villages (Tahar Valley) }\end{array}$ & 1000 & 29.06 .2014 & $\begin{array}{l}\text { Dried river } \\
\text { bad }\end{array}$ & & Ir.-Tur. \\
\hline \multirow{2}{*}{ Phlomis armeniaca Willd. } & 5340 & $\begin{array}{l}\text { B7 Tunceli: Nazımiye, } 3 \mathrm{~km} \text { from Nazımiye } \\
\text { to Dereova }\end{array}$ & 1600 & 20.07 .2014 & \multirow{2}{*}{ Steppe } & & \multirow{2}{*}{ Ir.-Tur. } \\
\hline & 5007 & $\begin{array}{l}\text { B7 Tunceli: Ovacık, } 5 \mathrm{~km} \text { from Eğripınar to } \\
\text { Yenikonak }\end{array}$ & 1480 & 20.06 .2014 & & & \\
\hline \multirow{8}{*}{ Phlomis kurdica Rech.f. } & 5101 & $\begin{array}{l}\text { B7 Tunceli: Çemişgezek, } 4 \mathrm{~km} \text { from Vişneli } \\
\text { to Arpaderen }\end{array}$ & 1110 & 21.06 .2014 & \multirow{8}{*}{ Steppe } & & \multirow{8}{*}{ Ir.-Tur. } \\
\hline & 5142 & $\begin{array}{l}\text { B7 Tunceli: Çemişgezek, W of Bozağaç } \\
\text { village }\end{array}$ & 1120 & 28.06.2014 & & & \\
\hline & 4088 & $\begin{array}{l}\text { B7 Tunceli: Mazgirt, between Tunceli and } \\
\text { Kovancılar, around of Kuşcu }\end{array}$ & 867 & 02.06 .2014 & & & \\
\hline & 6043 & $\begin{array}{l}\text { B7 Tunceli: Mazgirt, } 5.5 \mathrm{~km} \text { from Kale to } \\
\text { Doludizgin }\end{array}$ & 1560 & 15.08 .2014 & & & \\
\hline & 4512 & B7 Tunceli: Mazgirt, 1 km W of Kayac1 v. & 990 & 16.06 .2014 & & & \\
\hline & 4484 & B7 Tunceli: Pertek, 1 km NE of Akdemir v. & 1153 & 06.06 .2014 & & & \\
\hline & 4499 & $\begin{array}{l}\text { B7 Tunceli: Pertek, between Çemişgezek and } \\
\text { Elazı ğ, } 10 \mathrm{~km} \text { from Bulgurtepe to Payamdüzü }\end{array}$ & 1070 & 06.06 .2014 & & & \\
\hline & 5059 & $\begin{array}{l}\text { B7 Tunceli: Pertek, } 17 \mathrm{~km} \text { from Pertek to } \\
\text { Tunceli }\end{array}$ & 1300 & 21.06 .2014 & & & \\
\hline $\begin{array}{l}\text { Phlomis oppositiflora Boiss. \& } \\
\text { Hausskn }\end{array}$ & 5161 & $\begin{array}{l}\text { B7 Tunceli: Çemişgezek, between Anıl and } \\
\text { Gözlüçayır villages (Tahar Valley) }\end{array}$ & 1000 & 29.06 .2014 & Steppe & $*$ & Ir.-Tur. \\
\hline \multirow{2}{*}{$\begin{array}{l}\text { Phlomis pungens Willd. var. hirta } \\
\text { Velen. }\end{array}$} & 4120 & $\begin{array}{l}\text { B7 Tunceli: Mazgirt, Akpazar, S of Yenice v. } \\
\text { (N of Şevki mezrası) }\end{array}$ & 1160 & 02.06 .2014 & \multirow{2}{*}{ Steppe } & & \multirow{2}{*}{ Unk. } \\
\hline & 5341 & $\begin{array}{l}\text { B7 Tunceli: Nazımiye, } 3 \mathrm{~km} \text { from Nazımiye } \\
\text { to Dereova }\end{array}$ & 1600 & 20.07 .2014 & & & \\
\hline $\begin{array}{l}\text { Phlomis pungens Willd. var. } \\
\text { hispida Hub.-Mor. }\end{array}$ & 4595 & $\begin{array}{l}\text { B7 Tunceli: Mazgirt, } 2 \mathrm{~km} \text { from Akdüven to } \\
\text { Güleç }\end{array}$ & 1125 & 16.06.2014 & Steppe & Rcd. & Unk. \\
\hline \multirow{3}{*}{ Phlomis rigida Labill. } & 4611 & $\begin{array}{l}\text { B7 Tunceli: Mazgirt, } 11 \mathrm{~km} \text { from Çukur to } \\
\text { Güleç }\end{array}$ & 1230 & 16.06.2014 & \multirow{3}{*}{ Steppe } & & \multirow{3}{*}{ Ir.-Tur. } \\
\hline & 4433 & $\begin{array}{l}\text { B7 Tunceli: Center, } 1.5 \mathrm{~km} \text { from Aktuluk to } \\
\text { Buğulu }\end{array}$ & 1060 & 05.06 .2014 & & & \\
\hline & 5339 & $\begin{array}{l}\text { B7 Tunceli: Nazımiye, } 3 \mathrm{~km} \text { from Nazımiye } \\
\text { to Dereova }\end{array}$ & 1600 & 20.07.2014 & & & \\
\hline Phlomis tuberosa L. & 4751 & B7 Tunceli: Ovacık, $3 \mathrm{~km} \mathrm{~S}$ of Ovacık & 1315 & 17.06 .2014 & Steppe & & Unk. \\
\hline Prunella vulgaris L. & 5509 & $\begin{array}{l}\text { B8 Tunceli: Pülümür, between Bardakçı to } \\
\text { Derindere }\end{array}$ & 1980 & 22.07 .2014 & $\begin{array}{l}\text { Wet } \\
\text { meadows }\end{array}$ & & $\begin{array}{l}\text { Euro.- } \\
\text { Sib. }\end{array}$ \\
\hline Salvia adenocaulon P.H.Davis & 5446 & $\begin{array}{l}\text { B7 Tunceli: Hozat, N of Boydaş v. (Tahar } \\
\text { Valley) }\end{array}$ & 1215 & 21.07 .2014 & Steppe & $\begin{array}{l}* \\
\text { Rcd. }\end{array}$ & Medit. \\
\hline \multirow{2}{*}{ Salvia aethiopis $\mathrm{L}}$. & 4090 & $\begin{array}{l}\text { B7 Tunceli: Mazgirt, between Tunceli and } \\
\text { Kovancilar, around of Kuşcu }\end{array}$ & 867 & 02.06 .2014 & \multirow{2}{*}{ Steppe } & \multirow{2}{*}{ Rcd. } & \\
\hline & 4145 & $\begin{array}{l}\text { B7 Tunceli: Mazgirt, } 6 \mathrm{~km} \text { from Kizılcık to } \\
\text { Göktepe }\end{array}$ & 860 & 02.06 .2014 & & & Unk. \\
\hline $\begin{array}{l}\text { Salvia brachyantha (Bordz.) } \\
\text { Pobed. }\end{array}$ & 4314 & B7 Tunceli: Ovacık, 1.5 km E of Işıkvuran v. & 1960 & 04.06 .2014 & Steppe & Rcd. & Ir.-Tur. \\
\hline Salvia caespitosa Montbret \& & 4876 & $\begin{array}{l}\text { B7 Tunceli: Pülümür, } 11 \text { km NW of Ardıçlı } \\
\text { (Gersunut) v. (Munzur Mt.) }\end{array}$ & 2510 & 18.06 .2014 & & & \\
\hline Aucher ex Benth. & 6676 & $\begin{array}{l}\text { B7 Tunceli: Pülümür, } 12 \text { km NW of Ardıçlı } \\
\text { (Gersunut) v. (Munzur Mt.) }\end{array}$ & 2536 & 16.06.2015 & Mit. steppe & Rcd. & Ir.-1 ur. \\
\hline Salvia candidissima Vahl subsp. & 5291 & $\begin{array}{l}\text { B7 Tunceli: Center, } 10 \mathrm{~km} \text { from Tunceli to } \\
\text { Pülümür (Pülümür Valley) }\end{array}$ & 943 & 20.07.2014 & & & \\
\hline candidissima & 5104 & $\begin{array}{l}\text { B7 Tunceli: Çemişgezek, } 3 \mathrm{~km} \text { from } \\
\text { Arpaderen to Büyükörünce }\end{array}$ & 1112 & 21.06 .2014 & Steppe & & Iur. \\
\hline
\end{tabular}




\begin{tabular}{|c|c|c|c|c|c|c|c|}
\hline & 5215 & $\begin{array}{l}\text { B7 Tunceli: Nazımiye, } 800 \mathrm{~m} \text { from } \\
\text { Aşağırabat bridge to Büyükyurt (Hakis) v. }\end{array}$ & 1070 & 30.06 .2014 & & & \\
\hline & 5347 & B7 Tunceli: Nazımiye, E of Ramazanköy v. & 1440 & 20.07 .2014 & & & \\
\hline $\begin{array}{l}\text { Salvia candidissima Vahl subsp. } \\
\text { occidentalis Hedge }\end{array}$ & 4151 & $\begin{array}{l}\text { B7 Tunceli: Mazgirt, } 2 \mathrm{~km} \text { from } \\
\text { Beylermezrası to Aslanyurdu }\end{array}$ & 855 & 02.06 .2014 & Steppe & Rcd. & Unk. \\
\hline \multirow[b]{2}{*}{ Salvia cerino-pruinosa Rech.f. } & 4136 & $\begin{array}{l}\text { B7 Tunceli: Mazgirt, between Tunceli and } \\
\text { Kovancilar, junction of K1z1lc1k v. }\end{array}$ & 1060 & 02.06 .2014 & \multirow[b]{2}{*}{ Steppe } & \multirow{2}{*}{$\begin{array}{l}* \\
\text { Rcd. }\end{array}$} & \multirow{2}{*}{ Ir.-Tur. } \\
\hline & 3924 & $\begin{array}{l}\text { B7 Tunceli: Pertek, } 9 \mathrm{~km} \text { from Pertek to } \\
\text { Tunceli }\end{array}$ & 1395 & 24.05 .2014 & & & \\
\hline $\begin{array}{l}\text { Salvia euphratica Montbret \& } \\
\text { Aucher. var. leiocalycina } \\
\text { (Rech.f.) Hedge }\end{array}$ & 7512 & $\begin{array}{l}\text { B7 Tunceli: Mazgirt, 5.5. km from Kizılcık to } \\
\text { Karayusuf }\end{array}$ & 1270 & 03.06 .2017 & Steppe & Rcd. & Ir.-Tur. \\
\hline \multirow{2}{*}{ Salvia frigida Boiss. } & 4259 & $\begin{array}{l}\text { B7 Tunceli: Ovacık, } 3 \text { km N of Işıkvuran v. } \\
\text { Munzur Mt. }\end{array}$ & 2100 & 04.06 .2014 & \multirow{2}{*}{$\begin{array}{l}\text { Mt. steppe, } \\
\text { degraded } \\
\text { oak forest }\end{array}$} & & \multirow{2}{*}{ Ir.-Tur. } \\
\hline & 5543 & $\begin{array}{l}\text { B8 Tunceli: Pülümür, 1-2 km E of Bağırpaşa } \\
\text { Mt., } 2 \text { km N of Şampaşakaraderbent v. }\end{array}$ & 1886 & 22.07 .2014 & & & \\
\hline Salvia limbata C.A.Mey. & 4937 & $\begin{array}{l}\text { B7 Tunceli: Ovacık, } 4 \mathrm{~km} \text { from Eskigedik to } \\
\text { Issıkvuran }\end{array}$ & 1890 & 19.06.2014 & Steppe & Rcd. & Ir.-Tur. \\
\hline $\begin{array}{l}\text { Salvia macrochlamys Boiss. \& } \\
\text { Kotschy }\end{array}$ & 5694 & $\begin{array}{l}\text { B7 Tunceli: Center, } 8 \mathrm{~km} \text { from Tunceli to } \\
\text { Ovacik (Munzur valley) }\end{array}$ & 955 & 24.07 .2014 & Steppe & & Ir.-Tur. \\
\hline $\begin{array}{l}\text { Salvia microstegia Boiss. \& } \\
\text { Balansa }\end{array}$ & 5473 & $\begin{array}{l}\text { B8 Tunceli: Pülümür, between Derindere and } \\
\text { Bardakçı }\end{array}$ & 1840 & 22.07 .2014 & Steppe & & Ir.-Tur. \\
\hline \multirow{6}{*}{ Salvia multicaulis Vahl } & 3691 & $\begin{array}{l}\text { B7 Tunceli: Center, Tunceli - Elazı̆̆ road, } \\
700 \text { m after Aktuluk v. }\end{array}$ & 930 & 22.05 .2014 & \multirow{6}{*}{$\begin{array}{l}\text { Degraded } \\
\text { oak forest, } \\
\text { steppe }\end{array}$} & & \multirow{6}{*}{ Ir.-Tur. } \\
\hline & 4302 & $\begin{array}{l}\text { B7 Tunceli: Ovacık, } 13 \mathrm{~km} \text { from Işıkvuran to } \\
\text { Ağaçpınar }\end{array}$ & 1875 & 04.06 .2014 & & & \\
\hline & 4962 & $\begin{array}{l}\text { B7 Tunceli: Ovacık, N of Eskigedik v., } \\
\text { Munzur Mt. }\end{array}$ & 2160 & 19.06 .2014 & & & \\
\hline & 4252 & $\begin{array}{l}\text { B7 Tunceli: Ovacık, } 3 \text { km N of Işıkvuran v. } \\
\text { Munzur Mt. }\end{array}$ & 2020 & 04.06 .2014 & & & \\
\hline & 4267 & $\begin{array}{l}\text { B7 Tunceli: Ovacık, } 3 \text { km N of Işıkvuran v. } \\
\text { Munzur Mt. }\end{array}$ & 2100 & 04.06 .2014 & & & \\
\hline & 6399 & B7 Tunceli: Center, Alibaba nh. & 930 & 11.04 .2015 & & & \\
\hline \multirow{3}{*}{ Salvia palaestina Benth. } & 4560 & $\begin{array}{l}\text { B7 Tunceli: Mazgirt, } 1 \mathrm{~km} \text { from Ortaharman } \\
\text { to Sülüntaş }\end{array}$ & 1220 & 16.06 .2014 & \multirow{3}{*}{ Steppe } & & \multirow{3}{*}{ Ir.-Tur. } \\
\hline & 5109 & $\begin{array}{l}\text { B7 Tunceli: Pertek, } 3 \text { km from Elazığ-Pertek- } \\
\text { Çemişgezek crossroads to Çemişgezek ( } 1 \mathrm{~km} \\
\text { S of Çorovan v.) }\end{array}$ & 870 & 28.06 .2014 & & & \\
\hline & 5073 & $\begin{array}{l}\text { B7 Tunceli: Pertek, } 14 \mathrm{~km} \text { from Pertek to } \\
\text { Çemişgezek, } 2 \mathrm{~km} \text { after Singeç bridge, on the } \\
\text { right hills }\end{array}$ & 860 & 21.06 .2014 & & & \\
\hline \multirow{3}{*}{ Salvia recognita Fisch. C.A.Mey. } & 4598 & $\begin{array}{l}\text { B7 Tunceli: Mazgirt, } 2 \mathrm{~km} \text { from Akdüven to } \\
\text { Güleç }\end{array}$ & 1125 & 16.06 .2014 & \multirow{3}{*}{ Steppe } & \multirow{3}{*}{$*$} & \multirow{3}{*}{ Ir.-Tur. } \\
\hline & 5258 & $\begin{array}{l}\text { B7 Tunceli: Nazımiye, Pülümür Valley, E } \\
\text { slopes of Hılbeşen bridge, across of Ağlayan } \\
\text { Kayalar }\end{array}$ & 1345 & 30.06 .2014 & & & \\
\hline & 6479 & $\begin{array}{l}\text { B7 Tunceli: Pülümür, } 45 \mathrm{~km} \text { from Tunceli to } \\
\text { Pülümür (Pülümür Valley) }\end{array}$ & 1194 & 29.05 .2015 & & & \\
\hline Salvia russellii Benth. & 4551 & $\begin{array}{l}\text { B7 Tunceli: Mazgirt, } 3.5 \mathrm{~km} \text { from } \\
\text { Ortaharman to Kayacı }\end{array}$ & 1000 & 16.06 .2014 & Steppe & & Ir.-Tur. \\
\hline \multirow{3}{*}{ Salvia sclarea $\mathrm{L}$. } & 4153 & $\begin{array}{l}\text { B7 Tunceli: Mazgirt, } 2 \mathrm{~km} \text { from } \\
\text { Beylermezrası to Aslanyurdu }\end{array}$ & 855 & 02.06 .2014 & \multirow{3}{*}{$\begin{array}{l}\text { Steppe } \\
\text { scree }\end{array}$} & & \multirow{3}{*}{ Ir.-Tur. } \\
\hline & 3930 & B7 Tunceli: Center, Alibaba nh. & 930 & 25.05 .2014 & & & \\
\hline & 5640 & $\begin{array}{l}\text { B7 Tunceli: Ovacık, } 30 \mathrm{~km} \text { from Tunceli to } \\
\text { Ovacık (Munzur Valley) }\end{array}$ & 1070 & 24.07 .2014 & & & \\
\hline $\begin{array}{l}\text { Salvia staminea Montbret \& } \\
\text { Aucher ex Benth. }\end{array}$ & 6498 & $\begin{array}{l}\text { B7 Tunceli: Pülümür, } 1.8 \mathrm{~km} \text { from Hiver to } \\
\text { Dağyolu (Pülümür Mt.) }\end{array}$ & 1891 & 29.05 .2015 & Steppe & & Ir.-Tur. \\
\hline \multirow[t]{2}{*}{ Salvia syriaca $\mathrm{L}$. } & 4073 & $\begin{array}{l}\text { B7 Tunceli: Mazgirt, between Tunceli and } \\
\text { Kovancılar, around of Kușcu }\end{array}$ & 867 & 02.06 .2014 & \multirow{2}{*}{$\begin{array}{l}\text { Field side, } \\
\text { steppe }\end{array}$} & \multirow[t]{2}{*}{ Rcd. } & \multirow[t]{2}{*}{ Ir.-Tur. } \\
\hline & 3741 & B7 Tunceli: Center, $1 \mathrm{~km} \mathrm{~S}$ of Aktuluk & 930 & 22.05 .2014 & & & \\
\hline Salvia tomentosa Mill. & 5442 & $\begin{array}{l}\text { B7 Tunceli: Hozat, between Boydaş and } \\
\text { Karaçavuş }\end{array}$ & 1602 & 21.07.2014 & Steppe & & Medit. \\
\hline Salvia trichoclada Benth. & 3706 & $\begin{array}{l}\text { B7 Tunceli: Center, between Tunceli and } \\
\text { Pertek, junction of Kopuzlar v. }\end{array}$ & 1020 & 22.05 .2014 & Steppe & & Ir.-Tur. \\
\hline \multirow{5}{*}{$\begin{array}{l}\text { Salvia verticillata } \mathrm{L} \text {. subsp. } \\
\text { amasiaca (Freyn \& Bornm.) } \\
\text { Bornm. }\end{array}$} & 6175 & $\begin{array}{l}\text { B7 Tunceli: } 10 \mathrm{~km} \text { from Pülümür - Erzincan } \\
\text { mainroad to Süleymanuşağı village }\end{array}$ & 2210 & 27.08 .2014 & \multirow{5}{*}{$\begin{array}{l}\text { Steppe, } \\
\text { damp } \\
\text { places }\end{array}$} & & \\
\hline & 5450 & $\begin{array}{l}\text { B7 Tunceli: Hozat, N of Boydaş v. (Tahar } \\
\text { Valley) }\end{array}$ & 1215 & 21.07 .2014 & & & \\
\hline & 5219 & $\begin{array}{l}\text { B7 Tunceli: Nazımiye, } 800 \mathrm{~m} \text { from } \\
\text { Aşağırabat bridge to Büyükyurt (Hakis) v. }\end{array}$ & 1070 & 30.06 .2014 & & & Ir.-Tur. \\
\hline & 5057 & $\begin{array}{l}\text { B7 Tunceli: Ovacık, between Yarımkaya and } \\
\text { Şahverdi }\end{array}$ & 1485 & 20.06 .2014 & & & \\
\hline & 5756 & $\begin{array}{l}\text { B7 Tunceli: Pülümür, } 45 \mathrm{~km} \text { from Tunceli to } \\
\text { Pülümür (Pülümür valley) }\end{array}$ & 1200 & 06.08 .2014 & & & \\
\hline Salvia virgata Jacq. & 6174 & B7 Tunceli: 10 km from Pülümür - Erzincan & 2210 & 27.08 .2014 & Steppe & & Ir.-Tur. \\
\hline
\end{tabular}




\begin{tabular}{|c|c|c|c|c|c|c|c|}
\hline & & mainroad to Süleymanuşağ1 village & & & & & \\
\hline & 5740 & $\begin{array}{l}\text { B7 Tunceli: Mazgirt, } 3 \mathrm{~km} \text { from Akdüven to } \\
\text { Güleç }\end{array}$ & 1102 & 05.08 .2014 & & & \\
\hline & 5356 & B7 Tunceli: Nazımiye, Sarıyayla v. & 1835 & 20.07 .2014 & & & \\
\hline & $\mathbf{5 3 5 7}$ & B7 Tunceli: Nazımiye, Sarıyayla v. & 1835 & 20.07 .2014 & & & \\
\hline & 6017 & B8 Tunceli: Pülümür, 2 km W of Ağaşenliği & 1740 & 14.08.2014 & & & \\
\hline \multirow{7}{*}{ Satureja hortensis L. } & 5308 & $\begin{array}{l}\text { B7 Tunceli: Nazımiye, } 3 \mathrm{~km} \text { from Tunceli- } \\
\text { Pülümür-Nazımiye crossroads to Nazımiye }\end{array}$ & 1190 & 20.07.2014 & \multirow{7}{*}{$\begin{array}{l}\text { Ruderal, } \\
\text { steppe }\end{array}$} & & \multirow{7}{*}{ Unk. } \\
\hline & 6334 & $\begin{array}{l}\text { B7 Tunceli: Ovacık, } 2 \text { km from Tunceli- } \\
\text { Ovacık-Aşağıtorunoba crossroads to } \\
\text { Aşağıtorunoba }\end{array}$ & 1345 & 21.09.2014 & & & \\
\hline & 6217 & $\begin{array}{l}\text { B7 Tunceli: Ovacık, } 34 \mathrm{~km} \text { from Tunceli to } \\
\text { Ovacık (Munzur Valley) }\end{array}$ & 1127 & 28.08 .2014 & & & \\
\hline & 5902 & $\begin{array}{l}\text { B7 Tunceli: Ovacık, Tunceli-Ovacık- } \\
\text { Yakatarla junction }\end{array}$ & 1240 & 08.08 .2014 & & & \\
\hline & 6117 & B7 Tunceli: Pertek, 2 km W of Bulgurtepe v. & 1060 & 17.08 .2014 & & & \\
\hline & 6316 & $\begin{array}{l}\text { B7 Tunceli: Pülümür, } 3.5 \mathrm{~km} \text { from Pülümür } \\
\text { to Çobanyıldızı v. }\end{array}$ & 1395 & 20.09 .2014 & & & \\
\hline & 6033 & B8 Tunceli: Pülümür, SE of Altınhüseyin & 1630 & 14.08 .2014 & & & \\
\hline $\begin{array}{l}\text { Scutellaria albida } \text { L. subsp. } \\
\text { condensata (Rech.f.) J.R.Edm. }\end{array}$ & 4622 & $\begin{array}{l}\text { B7 Tunceli: Mazgirt, } 5 \mathrm{~km} \text { from Çukur to } \\
\text { Güleç }\end{array}$ & 1430 & 16.06 .2014 & $\begin{array}{l}\text { Steppe, } \\
\text { ruderal }\end{array}$ & Rcd. & Ir.-Tur. \\
\hline $\begin{array}{l}\text { Scutellaria albida L. subsp. } \\
\text { velenovskyi (Rech.f.) Greuter \& } \\
\text { Burdet }\end{array}$ & 4706 & $\begin{array}{l}\text { B7 Tunceli: Hozat, between Hozat and } \\
\text { Ovacık, around of Yüceldi v. }\end{array}$ & 1930 & 17.06 .2014 & Steppe & Rcd. & E.Medit. \\
\hline Scutellaria megalaspis Rech.f. & 5240 & $\begin{array}{l}\text { B7 Tunceli: Nazımiye, } 800 \mathrm{~m} \text { from } \\
\text { Aşağırabat bridge to Büyükyurt (Hakis) v. }\end{array}$ & 1070 & 30.06 .2014 & Steppe & & Ir.-Tur. \\
\hline $\begin{array}{l}\text { Scutellaria orientalis L. subsp. } \\
\text { bicolor (Hochst.) J.R.Edm. }\end{array}$ & 5266 & B7 Tunceli: Pertek, Camikebir nh. & 1100 & 19.07.2014 & $\begin{array}{l}\text { Rocky } \\
\text { slopes }\end{array}$ & $*$ & Ir.-Tur. \\
\hline \multirow{5}{*}{$\begin{array}{l}\text { Scutellaria orientalis L. subsp. } \\
\text { haussknechtii (Boiss.) J.R.Edm. }\end{array}$} & 4517 & \begin{tabular}{|l|} 
B7 Tunceli: Mazgirt, 1 km W of Kayacı v. \\
\end{tabular} & 990 & 16.06 .2014 & \multirow{5}{*}{$\begin{array}{l}\text { Steppe, } \\
\text { rocky } \\
\text { areas }\end{array}$} & \multirow{5}{*}{$*$} & \multirow{5}{*}{ Ir.-Tur. } \\
\hline & 3927 & B7 Tunceli: Center, Alibaba nh. & 930 & 25.05 .2014 & & & \\
\hline & 3905 & $\begin{array}{l}\text { B7 Tunceli: Pertek, } 12 \mathrm{~km} \text { from Pertek to } \\
\text { Çemişgezek, around of Singeç bridge }\end{array}$ & 855 & 24.05 .2014 & & & \\
\hline & 3752 & $\begin{array}{l}\text { B7 Tunceli: Center, } 1 \mathrm{~km} \text { from Kopuzlar to } \\
\text { Yolkonak }\end{array}$ & 1114 & 22.05 .2014 & & & \\
\hline & 3753 & $\begin{array}{l}\text { B7 Tunceli: Center, } 1 \mathrm{~km} \text { from Kopuzlar to } \\
\text { Yolkonak }\end{array}$ & 1114 & 22.05 .2014 & & & \\
\hline $\begin{array}{l}\text { Scutellaria orientalis L. subsp. } \\
\text { orientalis }\end{array}$ & 5052 & $\begin{array}{l}\text { B7 Tunceli: Ovacık, between Gözeler and } \\
\text { Yazıören, } 1 \mathrm{~km} \mathrm{~S} \text { of Mollaaliler v. } \\
\end{array}$ & 1380 & 20.06 .2014 & Steppe & & Ir.-Tur. \\
\hline $\begin{array}{l}\text { Scutellaria orientalis L. subsp. } \\
\text { virens (Boiss. \& Kotschy) } \\
\text { J.R.Edm. }\end{array}$ & 5306 & $\begin{array}{l}\text { B7 Tunceli: Nazımiye, } 3 \mathrm{~km} \text { from Tunceli- } \\
\text { Pülümür-Nazımiye crossroads to Nazımiye }\end{array}$ & 1190 & 20.07 .2014 & Steppe & & Ir.-Tur. \\
\hline \multirow[b]{2}{*}{ Scutellaria salviifolia Benth. } & 3685 & $\begin{array}{l}\text { B7 Tunceli: Center, Tunceli - Elazı ğ road, } \\
700 \text { m after Aktuluk v. }\end{array}$ & 930 & 22.05 .2014 & \multirow[b]{2}{*}{ Steppe } & \multirow[b]{2}{*}{ Red. } & \multirow[b]{2}{*}{ Unk. } \\
\hline & 4159 & $\begin{array}{l}\text { B7 Tunceli: Nazımiye, } 6.5 \mathrm{~km} \text { from } \\
\text { Nazımiye to Tunceli, } 400 \mathrm{~m} \text { after Geriş v. } \\
\text { junction }\end{array}$ & 1340 & 03.06 .2014 & & & \\
\hline $\begin{array}{l}\text { Stachys balansae Boiss. \& } \\
\text { Kotschy }\end{array}$ & 5878 & $\begin{array}{l}\text { B7 Tunceli: Nazımiye, } 6 \mathrm{~km} \mathrm{NE} \text { of } \\
\text { Büyükyurt (Hakis) v. }\end{array}$ & 2050 & 07.08 .2014 & Steppe & $*$ & E.Medit. \\
\hline \multirow[b]{2}{*}{$\begin{array}{l}\text { Stachys burgsdorffioides (Benth.) } \\
\text { Boiss. }\end{array}$} & 4967 & $\begin{array}{l}\text { B7 Tunceli: Ovacık, N of Eskigedik v., } \\
\text { Munzur Mt. }\end{array}$ & 2160 & 19.06.2014 & \multirow[b]{2}{*}{ Steppe } & & \multirow[b]{2}{*}{ Ir.-Tur. } \\
\hline & 4187 & $\begin{array}{l}\text { B7 Tunceli: Center, } 29 \mathrm{~km} \text { from Tunceli to } \\
\text { Pülümür (Pülümür Valley), } 650 \mathrm{~m} \mathrm{NW} \text { of } \\
\text { Kutudere bridge }\end{array}$ & 1070 & 03.06 .2014 & & & \\
\hline $\begin{array}{l}\text { Stachys cretica L. subsp. } \\
\text { anatolica } \text { Rech.f. }\end{array}$ & 5801 & $\begin{array}{l}\text { B7 Tunceli: Ovacik, } 9.5 \mathrm{~km} \text { from Kocatepe to } \\
\text { Yalmanlar (the walk way of Buyer Baba } \\
\text { sanctuary) }\end{array}$ & 2600 & 06.08 .2014 & Steppe & $*$ & Unk. \\
\hline \multirow[b]{2}{*}{$\begin{array}{l}\text { Stachys iberica M.Bieb. subsp. } \\
\text { stenostachya (Boiss.) Rech.f. }\end{array}$} & 5328 & $\begin{array}{l}\text { B7 Tunceli: Nazımiye, } 3 \mathrm{~km} \text { from Nazımiye } \\
\text { to Dereova }\end{array}$ & 1600 & 20.07 .2014 & \multirow[b]{2}{*}{ Steppe } & & \multirow[b]{2}{*}{ Ir.-Tur. } \\
\hline & 4160 & $\begin{array}{l}\text { B7 Tunceli: Nazımiye, } 6.5 \mathrm{~km} \text { from } \\
\text { Nazımiye to Tunceli, } 400 \mathrm{~m} \text { after Geriş v. } \\
\text { junction }\end{array}$ & 1340 & 03.06 .2014 & & & \\
\hline \multirow{2}{*}{ Stachys lavandulifolia Vahl } & 4034 & $\begin{array}{l}\text { B7 Tunceli: Ovacık, } 41 \mathrm{~km} \text { from Tunceli to } \\
\text { Ovacık (Munzur Valley) }\end{array}$ & 1175 & 26.05 .2014 & \multirow{2}{*}{ Steppe } & & \multirow{2}{*}{ Unk. } \\
\hline & 3852 & $\begin{array}{l}\text { B7 Tunceli: Hozat, } 3.5 \mathrm{~km} \text { from Demirkapı to } \\
\text { Dervişcemal }\end{array}$ & 1760 & 23.05 .2014 & & & \\
\hline $\begin{array}{l}\text { Stachys melampyroides Hand.- } \\
\text { Mazz. }\end{array}$ & 4544 & B7 Tunceli: Mazgirt, 1 km W of Kayacı v. & 985 & 16.06 .2014 & Steppe & Rcd. & Ir.-Tur. \\
\hline \multirow{2}{*}{$\begin{array}{l}\text { Stachys saturejoides Montbret \& } \\
\text { Aucher ex Benth. }\end{array}$} & 3913 & B7 Tunceli: Pertek, Camikebir nh. & 1090 & 24.05 .2014 & \multirow[b]{2}{*}{ Steppe } & \multirow[b]{2}{*}{ Rcd. } & \multirow[b]{2}{*}{ Ir.-Tur. } \\
\hline & 3890 & $\begin{array}{l}\text { B7 Tunceli: Pertek, } 9 \mathrm{~km} \text { from Pertek to } \\
\text { Çemişgezek, } 2.5 \mathrm{~km} \text { before Singeç bridge }\end{array}$ & 865 & 24.05 .2014 & & & \\
\hline $\begin{array}{l}\text { Stachys setifera C.A.Mey. subsp. } \\
\text { lycia (Gand.) R.Bhattacharjee }\end{array}$ & 5663 & $\begin{array}{l}\text { B7 Tunceli: Ovacık, } 10 \mathrm{~km} \text { from Eğripınar to } \\
\text { Yenikonak }\end{array}$ & 1650 & 24.07 .2014 & Steppe & Rcd. & Ir.-Tur. \\
\hline $\begin{array}{l}\text { Stachys spectabilis Choisy ex } \\
\text { DC. }\end{array}$ & 5427 & $\begin{array}{l}\text { B7 Tunceli: Hozat, between Karaçavuş and } \\
\text { Boydaş }\end{array}$ & 1720 & 21.07.2014 & Steppe & & Ir.-Tur. \\
\hline
\end{tabular}




\begin{tabular}{|c|c|c|c|c|c|c|c|}
\hline & 5616 & B7 Tunceli: Nazımiye, $1 \mathrm{~km} \mathrm{~S}$ of Holik v. & 1875 & 23.07 .2014 & & & \\
\hline & 6078 & $\begin{array}{l}\text { B8 Tunceli: Pülümür, } 3 \mathrm{~km} \text { from Hasangazi } \\
\text { to Kırklar }\end{array}$ & 1890 & 16.08 .2014 & & & \\
\hline & 6093 & B8 Tunceli: Pülümür, 2.6 km E of Kırklar v. & 2120 & 16.08 .2014 & & & \\
\hline \multirow{5}{*}{$\begin{array}{l}\text { Stachys tundjeliensis Kit Tan \& } \\
\text { Sorger }\end{array}$} & 5404 & $\begin{array}{l}\text { B7 Tunceli: Hozat, } 1 \mathrm{~km} \text { from Demirkapı to } \\
\text { Dervişcemal }\end{array}$ & 1620 & 21.07 .2014 & \multirow{5}{*}{ Steppe } & \multirow{5}{*}{$*$} & \multirow{5}{*}{ Ir.-Tur. } \\
\hline & 3842 & $\begin{array}{l}\text { B7 Tunceli: Center, } 12 \mathrm{~km} \text { from Aktuluk to } \\
\text { Demirkap1 }\end{array}$ & 1430 & 23.05 .2014 & & & \\
\hline & 5619 & B7 Tunceli: Nazımiye, 1 km S of Holik v. & 1960 & 23.07 .2014 & & & \\
\hline & 5053 & $\begin{array}{l}\text { B7 Tunceli: Ovacık, between Gözeler and } \\
\text { Yazıören, } 1 \mathrm{~km} \text { S of Mollaaliler v. }\end{array}$ & 1380 & 20.06 .2014 & & & \\
\hline & 4037 & $\begin{array}{l}\text { B7 Tunceli: Ovacık, } 41 \mathrm{~km} \text { from Tunceli to } \\
\text { Ovacık (Munzur Valley) }\end{array}$ & 1175 & 26.05 .2014 & & & \\
\hline \multirow{3}{*}{$\begin{array}{l}\text { Teucrium chamaedrys L. subsp. } \\
\text { sinuatum (Celak.) Rech.f. }\end{array}$} & 5239 & $\begin{array}{l}\text { B7 Tunceli: Nazımiye, } 800 \mathrm{~m} \text { from } \\
\text { Aşağırabat bridge to Büyükyurt (Hakis) v. }\end{array}$ & 1070 & 30.06 .2014 & \multirow{3}{*}{$\begin{array}{l}\text { Rock } \\
\text { crevices, } \\
\text { steppe }\end{array}$} & & \multirow{3}{*}{ Ir.-Tur. } \\
\hline & 5331 & $\begin{array}{l}\text { B7 Tunceli: Nazımiye, } 3 \mathrm{~km} \text { from Nazımiye } \\
\text { to Dereova }\end{array}$ & 1600 & 20.07.2014 & & & \\
\hline & 5916 & $\begin{array}{l}\text { B7 Tunceli: Ovacık, } 3 \text { km N of Iş̧kvuran v. } \\
\text { Munzur Mt. }\end{array}$ & 2005 & 08.08 .2014 & & & \\
\hline $\begin{array}{l}\text { Teucrium chamaedrys L. subsp. } \\
\text { tauricola } \text { Rech.f. }\end{array}$ & 4977 & $\begin{array}{l}\text { B7 Tunceli: Center, } 2 \mathrm{~km} \text { from Sarıtaş to } \\
\text { Gözen (İksor), Kalan Valley }\end{array}$ & 1180 & 19.06.2014 & Steppe & Rcd. & Medit. \\
\hline $\begin{array}{l}\text { Teucrium leucophyllum Montbret } \\
\text { \& Aucher ex Benth. }\end{array}$ & 5201 & $\begin{array}{l}\text { B7 Tunceli: Çemişgezek, } 2 \mathrm{~km} \text { from } \\
\text { Dedebeyli to Akçayurt }\end{array}$ & 1100 & 29.06 .2014 & Scree & $*$ & Ir.-Tur. \\
\hline \multirow{6}{*}{$\begin{array}{l}\text { Teucrium multicaule Montbret \& } \\
\text { Aucher ex Benth. }\end{array}$} & 4125 & $\begin{array}{l}\text { B7 Tunceli: Mazgirt, Akpazar, S of Yenice v. } \\
\text { (N of Şevki mezrası) }\end{array}$ & 1250 & 02.06 .2014 & \multirow{6}{*}{ Steppe } & & \multirow{6}{*}{ Ir.-Tur. } \\
\hline & 3925 & B7 Tunceli: Center, Alibaba nh. & 930 & 25.05 .2014 & & & \\
\hline & 4430 & $\begin{array}{l}\text { B7 Tunceli: Pülümür, } 9 \mathrm{~km} \text { from Pülümür to } \\
\text { Tunceli (Pülümür valley), S of Kangallı v. }\end{array}$ & 1324 & 05.06 .2014 & & & \\
\hline & 4431 & $\begin{array}{l}\text { B7 Tunceli: Pülümür, } 9 \mathrm{~km} \text { from Pülümür to } \\
\text { Tunceli (Pülümür valley), S of Kangallı v. }\end{array}$ & 1324 & 05.06 .2014 & & & \\
\hline & 3750 & $\begin{array}{l}\text { B7 Tunceli: Center, } 1 \mathrm{~km} \text { from Kopuzlar to } \\
\text { Yolkonak }\end{array}$ & 1114 & 22.05 .2014 & & & \\
\hline & 6456 & $\begin{array}{l}\text { B7 Tunceli: Center, } 15 \mathrm{~km} \text { from Tunceli to } \\
\text { Pülümür (Pülümür Valley) }\end{array}$ & 1019 & 29.05 .2015 & & & \\
\hline \multirow{2}{*}{$\begin{array}{l}\text { Teucrium orientale L. var. } \\
\text { glabrescens Hausskn. ex Bornm. }\end{array}$} & $\mathbf{5 0 2 7}$ & $\begin{array}{l}\text { B7 Tunceli: Ovacık, } 10 \mathrm{~km} \text { from Eğripınar to } \\
\text { Yenikonak }\end{array}$ & 1570 & 20.06.2014 & \multirow{2}{*}{ Steppe } & & \multirow{2}{*}{ Ir.-Tur. } \\
\hline & 6104 & $\begin{array}{l}\text { B7 Tunceli: Pülümür, } 7 \mathrm{~km} \text { from Pülümür to } \\
\text { Ardıçlı (Gersunut) v. }\end{array}$ & 1690 & 16.08.2014 & & & \\
\hline \multirow{2}{*}{$\begin{array}{l}\text { Teucrium orientale } \mathrm{L} \text {. var. } \\
\text { orientale }\end{array}$} & 4207 & $\begin{array}{l}\text { B7 Tunceli: Ovacik, } 1.5 \mathrm{~km} \text { from Ovacik- } \\
\text { Tunceli-Yakatarla crossroads to Yakatarla v. }\end{array}$ & 1320 & 04.06 .2014 & \multirow{2}{*}{ Steppe } & & \multirow{2}{*}{ Ir.-Tur. } \\
\hline & 3774 & $\begin{array}{l}\text { B7 Tunceli: Center, } 500 \text { m from Tunceli- } \\
\text { Pülümür-Sütlüce crossroads to Sütlüce v. }\end{array}$ & 940 & 23.05.2014 & & & \\
\hline \multirow{2}{*}{$\begin{array}{l}\text { Teucrium orientale L. var. } \\
\text { puberulens Ekim }\end{array}$} & 6205 & $\begin{array}{l}\text { B7 Tunceli: Hozat, } 16 \mathrm{~km} \text { from Hozat to } \\
\text { Ovacık }\end{array}$ & 1940 & 28.08.2014 & \multirow{2}{*}{ Steppe } & & \multirow{2}{*}{ Ir.-Tur. } \\
\hline & 5018 & $\begin{array}{l}\text { B7 Tunceli: Ovacık, } 10 \mathrm{~km} \text { from Eğripınar to } \\
\text { Yenikonak }\end{array}$ & 1570 & 20.06.2014 & & & \\
\hline \multirow[b]{2}{*}{ Teucrium parviflorum Schreber } & 4520 & B7 Tunceli: Mazgirt, 1 km W of Kayacı v. & 990 & 16.06 .2014 & \multirow[b]{2}{*}{ Steppe } & & \multirow[b]{2}{*}{ Ir.-Tur. } \\
\hline & 4109 & $\begin{array}{l}\text { B7 Tunceli: Mazgirt, } 7 \mathrm{~km} \text { from Kızılcık to } \\
\text { Göktepe }\end{array}$ & 935 & 02.06 .2014 & & & \\
\hline Teucrium polium L. & 4521 & B7 Tunceli: Mazgirt, 1 km W of Kayacı v. & 990 & 16.06 .2014 & Steppe & & Unk. \\
\hline Thymus convolutus Klokov & 4901 & $\begin{array}{l}\text { B7 Tunceli: Pülümür, } 11 \text { km NW of Ardıçlı } \\
\text { (Gersunut) v. (Munzur Mt.) }\end{array}$ & 2380 & 18.06 .2014 & Steppe & $*$ & Ir.-Tur. \\
\hline Thymus fallax Fisch. \& C.A.Mey. & 5553 & B8 Tunceli: Pülümür, $5 \mathrm{~km} \mathrm{~S}$ of Derindere & 2030 & 22.07 .2014 & Steppe & & Ir.-Tur. \\
\hline \multirow{3}{*}{ Thymus haussknechtii Velen. } & 5108 & $\begin{array}{l}\text { B7 Tunceli: Çemişgezek, } 3 \mathrm{~km} \text { from Vişneli } \\
\text { to Çemişgezek }\end{array}$ & 880 & 21.06.2014 & \multirow{3}{*}{$\begin{array}{l}\text { Steppe, on } \\
\text { rocks }\end{array}$} & \multirow{3}{*}{$*$} & \\
\hline & 5159 & $\begin{array}{l}\text { B7 Tunceli: Çemişgezek, between Anıl and } \\
\text { Gözlüçayır villages (Tahar Valley) }\end{array}$ & 1000 & 29.06 .2014 & & & Ir.-Tur. \\
\hline & 3911 & $\begin{array}{l}\text { B7 Tunceli: Pertek, } 12 \mathrm{~km} \text { from Pertek to } \\
\text { Çemişgezek, around of Singeç bridge }\end{array}$ & 855 & 24.05 .2014 & & & \\
\hline & 5229 & $\begin{array}{l}\text { B7 Tunceli: Nazımiye, } 800 \mathrm{~m} \text { from } \\
\text { Aşağırabat bridge to Büyükyurt (Hakis) v. }\end{array}$ & 1070 & 30.06 .2014 & & & \\
\hline $\begin{array}{l}\text { Thymus kotschyanus Boiss. \& } \\
\text { Hohen. }\end{array}$ & 4210 & $\begin{array}{l}\text { B7 Tunceli: Ovacık, } 1.5 \mathrm{~km} \text { from Ovacık- } \\
\text { Tunceli-Yakatarla crossroads to Yakatarla v. }\end{array}$ & 1320 & 04.06 .2014 & Steppe & & Ir.-Tur. \\
\hline & 4769 & $\begin{array}{l}\text { B7 Tunceli: Pülümür, } 2 \mathrm{~km} \text { from Turnadere } \\
\text { to Közlüce }\end{array}$ & 1575 & 18.06 .2014 & & & \\
\hline Thym & 6648 & $\begin{array}{l}\text { B7 Tunceli: Pülümür, } 11 \text { km NW of Ardıçlı } \\
\text { (Gersunut) v. (Munzur Mt.) }\end{array}$ & 2204 & 27.06 .2015 & & & Unk \\
\hline Thymus leucotrlchus Hal. & 4845 & $\begin{array}{l}\text { B8 Tunceli: Pülümür, } 500 \mathrm{~m} \mathrm{~N} \text { of Balpayam } \\
\text { (3-4 km S of Kırklar v.) }\end{array}$ & 2400 & 18.06.2014 & Steppe & & Unk. \\
\hline Thymus sipyleus Boiss. & 7513 & $\begin{array}{l}\text { B7 Tunceli: Mazgirt, 5.5. km from Kızılcık to } \\
\text { Karayusuf }\end{array}$ & 1270 & 03.06 .2017 & Steppe & Rcd. & Unk. \\
\hline & 3684 & $\begin{array}{l}\text { B7 Tunceli: Center, Tunceli - Elazı ğ road, } \\
700 \text { m after Aktuluk v. }\end{array}$ & 930 & 22.05 .2014 & & & \\
\hline Zizıphora capitata $\mathrm{L}$. & 3686 & $\begin{array}{l}\text { B7 Tunceli: Center, Tunceli - Elazığ road, } \\
700 \text { m after Aktuluk v. }\end{array}$ & 930 & 22.05 .2014 & Steppe & & Unk. \\
\hline
\end{tabular}




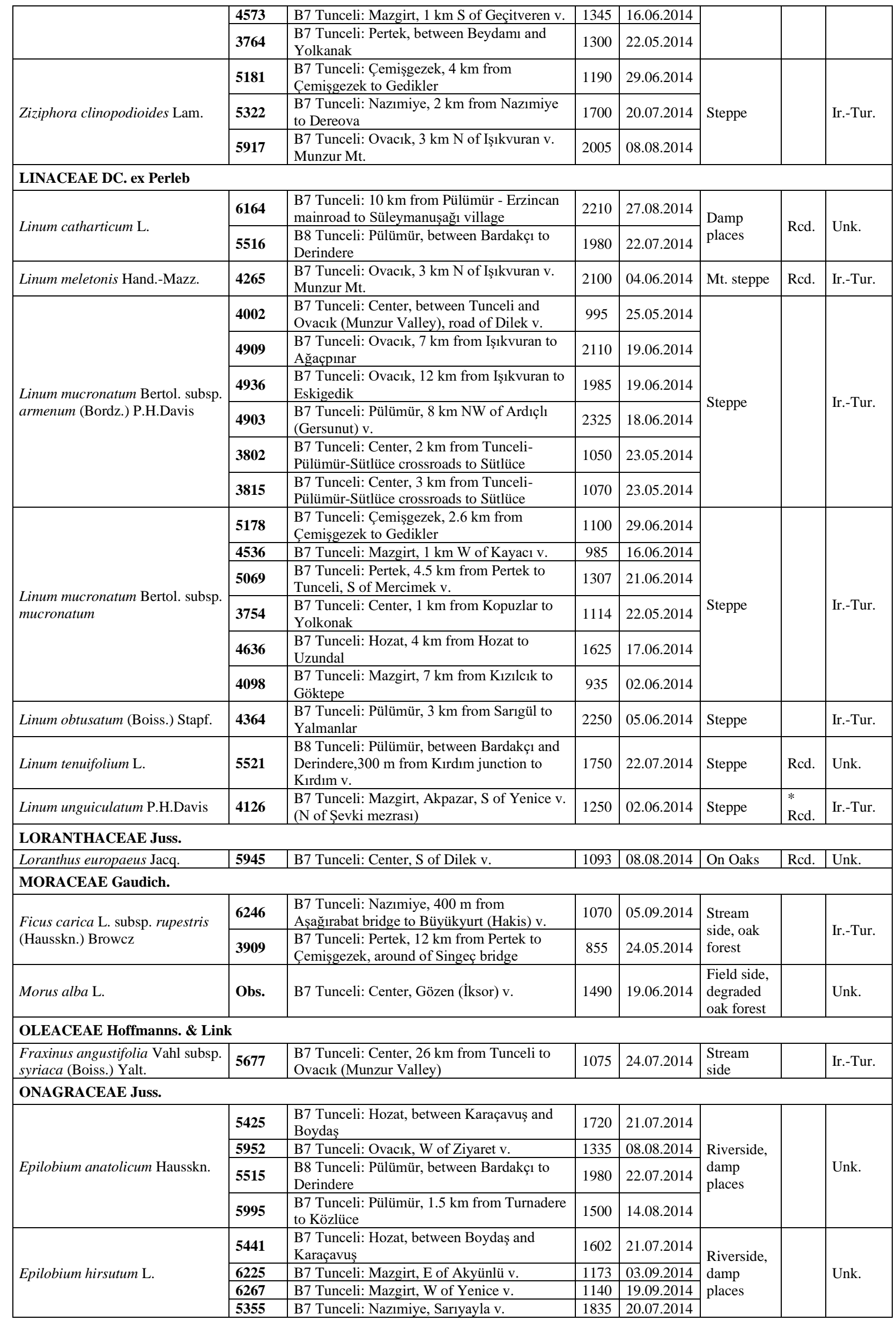




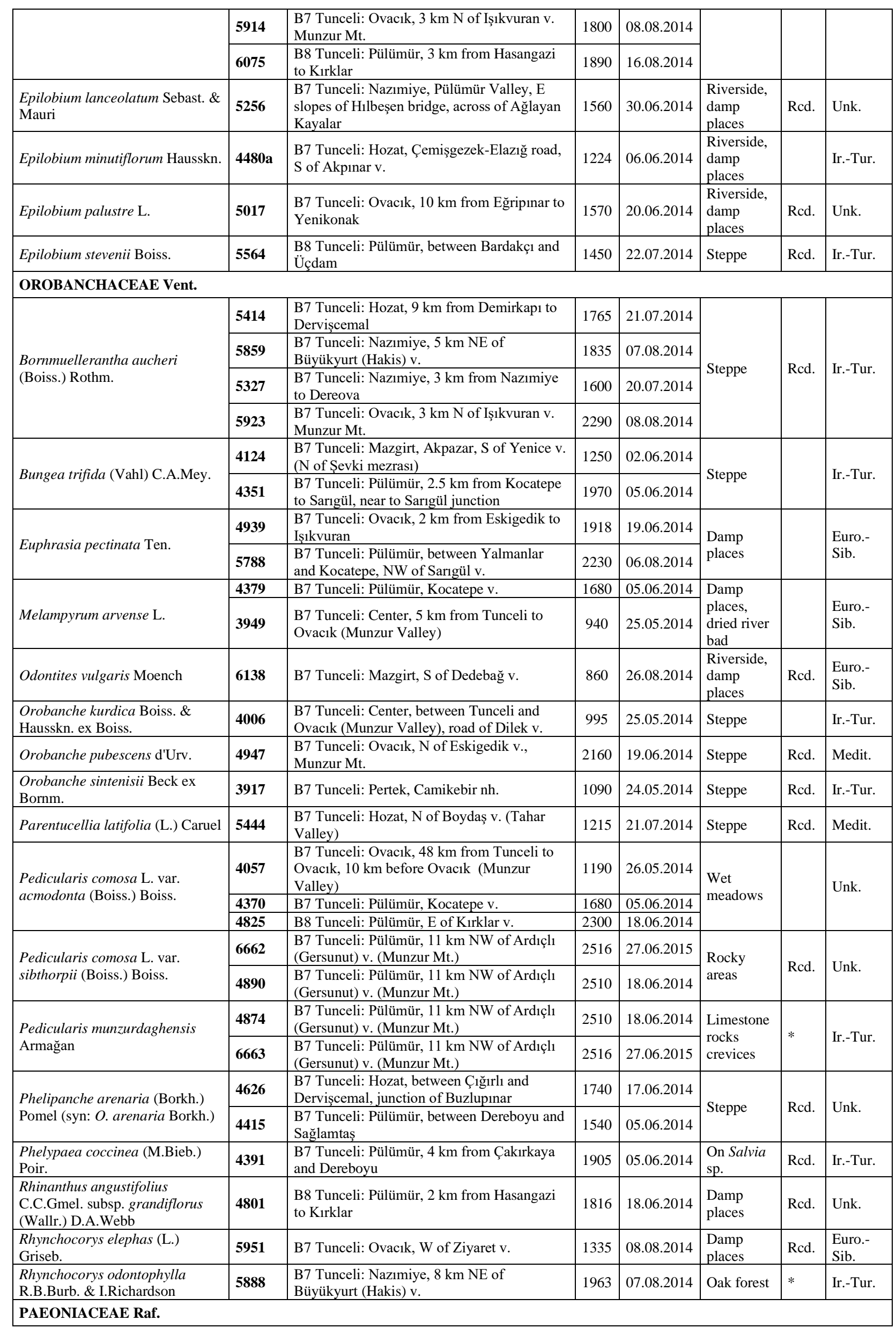




\begin{tabular}{|c|c|c|c|c|c|c|c|}
\hline \multirow{3}{*}{ Paeonia arietina G.Anderson } & 4457 & $\begin{array}{l}\text { B7 Tunceli: Hozat, between Demirkapı and } \\
\text { Dervișcemal, } 300 \mathrm{~m} \text { to Buzlupınar junction }\end{array}$ & \multirow{2}{*}{1810} & \multirow{2}{*}{\begin{tabular}{l|}
06.06 .2014 \\
04.06 .2014
\end{tabular}} & \multirow{3}{*}{$\begin{array}{l}\text { Steppe, } \\
\text { Oak forest }\end{array}$} & & \multirow{3}{*}{ Unk. } \\
\hline & 4293 & B7 Tunceli: Ovacık, Işıkvuran v. & & & & & \\
\hline & 4352 & $\begin{array}{l}\text { B7 Tunceli: Pülümür, } 2.5 \mathrm{~km} \text { from Kocatepe } \\
\text { to Sarıgül, near to Sarı̈ül junction }\end{array}$ & 1970 & 05.06 .2014 & & & \\
\hline \multicolumn{8}{|l|}{ PAPAVERACEAE Juss. } \\
\hline Fumaria asepala Boiss. & 3794 & $\begin{array}{l}\text { B7 Tunceli: Center, between Tunceli and } \\
\text { Sütlüce, } 1.8 \mathrm{~km} \text { from Sütlüce junction }\end{array}$ & 1030 & 23.05 .2014 & $\begin{array}{l}\text { Field side, } \\
\text { steppe }\end{array}$ & & Unk. \\
\hline Glaucium cappadocicum Boiss. & 6168 & $\begin{array}{l}\text { B7 Tunceli: } 10 \mathrm{~km} \text { from Pülümür - Erzincan } \\
\text { mainroad to Süleymanuşağı village }\end{array}$ & 2210 & 27.08 .2014 & Steppe & $\begin{array}{l}* \\
\text { Rcd. }\end{array}$ & Ir.-Tur. \\
\hline \multirow{2}{*}{ Glaucium leiocarpum Boiss. } & 6703 & $\begin{array}{l}\text { B7 Tunceli: Çemişgezek, } 1.5 \mathrm{~km} \mathrm{~W} \text { of } \\
\text { Sarıbalta v. }\end{array}$ & 1187 & 28.06 .2015 & \multirow{2}{*}{ Steppe } & & \multirow{2}{*}{ Unk. } \\
\hline & 4403 & $\begin{array}{l}\text { B7 Tunceli: Pülümür, between Dereboyu and } \\
\text { Sağlamtaş }\end{array}$ & 1610 & 05.06 .2014 & & & \\
\hline Hypecoum procumbens $\mathrm{L}$. & 4500 & $\begin{array}{l}\text { B7 Tunceli: Pertek, between Çemişgezek and } \\
\text { Elazı ğ, } 2 \mathrm{~km} \text { from Payamdüzü to Akçapınar }\end{array}$ & 945 & 06.06 .2014 & $\begin{array}{l}\text { Field side, } \\
\text { steppe }\end{array}$ & Rcd. & Unk. \\
\hline $\begin{array}{l}\text { Hypecoum pseudograndiflorum } \\
\text { Petrovic }\end{array}$ & 3792 & $\begin{array}{l}\text { B7 Tunceli: Center, between Tunceli and } \\
\text { Sütlüce, } 1.8 \mathrm{~km} \text { from Sütlüce junction }\end{array}$ & 1030 & 23.05 .2014 & $\begin{array}{l}\text { Field side, } \\
\text { steppe }\end{array}$ & Rcd. & Unk. \\
\hline Papaver armeniacum Lam. & 4188 & $\begin{array}{l}\text { B7 Tunceli: Ovacık, } 1.5 \mathrm{~km} \text { from Ovacık- } \\
\text { Tunceli-Yakatarla crossroads to Yakatarla v. }\end{array}$ & 1320 & 04.06 .2014 & Steppe & Rcd. & Ir.-Tur. \\
\hline \multirow[b]{2}{*}{ Papaver bracteatum Lindl. } & 4228 & $\begin{array}{l}\text { B7 Tunceli: Ovacık, } 8 \mathrm{~km} \text { from Ovacık- } \\
\text { Tunceli-Yakatarla to Yakatarla }\end{array}$ & 1680 & 04.06 .2014 & \multirow[b]{2}{*}{ Steppe } & & \multirow[b]{2}{*}{ Unk. } \\
\hline & 4051 & $\begin{array}{l}\text { B7 Tunceli: Ovacık, } 47 \mathrm{~km} \text { from Tunceli to } \\
\text { Ovacık, } 11 \mathrm{~km} \text { before Ovacık (Munzur } \\
\text { Valley) }\end{array}$ & 1200 & 26.05 .2014 & & & \\
\hline Papaver dubium L. & 3986 & $\begin{array}{l}\text { B7 Tunceli: Center, } 10 \mathrm{~km} \text { from Tunceli to } \\
\text { Ovacık (Munzur Valley) }\end{array}$ & 960 & 25.05 .2014 & Steppe & & Unk. \\
\hline \multirow{2}{*}{$\begin{array}{l}\text { Papaver macrostomum Boiss. \& } \\
\text { A.Huet }\end{array}$} & 3989 & $\begin{array}{l}\text { B7 Tunceli: Center, between Tunceli and } \\
\text { Ovacik (Munzur Valley), road of Dilek v. }\end{array}$ & 1043 & 25.05 .2014 & \multirow{2}{*}{ Steppe } & & \multirow{2}{*}{ Ir.-Tur. } \\
\hline & 4753 & $\begin{array}{l}\text { B7 Tunceli: Pülümür, } 1 \mathrm{~km} \text { from Pülümür- } \\
\text { Tunceli-Turnadere crossroads to Turnadere v. }\end{array}$ & 1455 & 18.06 .2014 & & & \\
\hline $\begin{array}{l}\text { Papaver persicum Lindl. subsp. } \\
\text { fulvum Kit Tan \& Sorger }\end{array}$ & 3962 & $\begin{array}{l}\text { B7 Tunceli: Center, } 7 \mathrm{~km} \text { from Tunceli to } \\
\text { Ovacık (Munzur Valley) }\end{array}$ & 960 & 25.05 .2014 & Steppe & $\begin{array}{l}* \\
\text { Rcd. }\end{array}$ & Unk. \\
\hline $\begin{array}{l}\text { Papaver persicum Lindl. subsp. } \\
\text { persicum }\end{array}$ & 4137 & $\begin{array}{l}\text { B7 Tunceli: Mazgirt, between Tunceli and } \\
\text { Kovancilar, junction of Kizllc1k v. }\end{array}$ & 1060 & 02.06 .2014 & Steppe & & Unk. \\
\hline Papaver rhoeas $\mathrm{L}$. & 3988 & $\begin{array}{l}\text { B7 Tunceli: Center, between Tunceli and } \\
\text { Ovacik (Munzur Valley), road of Dilek v. }\end{array}$ & 1043 & 25.05 .2014 & Steppe & & Unk. \\
\hline Roemeria hybrida (L.) DC. & 5156 & $\begin{array}{l}\text { B7 Tunceli: Çemişgezek, between Anıl and } \\
\text { Gözlüçayır villages (Tahar Valley) }\end{array}$ & 1000 & 29.06 .2014 & Steppe & & Unk. \\
\hline \multicolumn{8}{|l|}{ PEDALIACEAE R.Br. } \\
\hline Sesamum indicum $\mathrm{L}$. & 5969 & $\begin{array}{l}\text { B7 Tunceli: Pertek, } 3 \mathrm{~km} \text { from Geçityaka to } \\
\text { Dorutay }\end{array}$ & 1000 & 13.08 .2014 & Cultivate & & Unk. \\
\hline \multicolumn{8}{|l|}{ PHYLLANTHACEAE Martinov } \\
\hline Andrachne telephioides L. & 5965 & $\begin{array}{l}\text { B7 Tunceli: Pertek, } 9 \mathrm{~km} \text { from Pertek to } \\
\text { Çemişgezek, } 2.5 \mathrm{~km} \text { before Singeç bridge }\end{array}$ & 875 & 13.08 .2014 & Steppe & & Unk. \\
\hline \multicolumn{8}{|l|}{ PINACEAE Spreng. ex F.Rudolphi } \\
\hline $\begin{array}{l}\text { Pinus sylvestris L. var. hamata } \\
\text { Steven }\end{array}$ & 5522 & $\begin{array}{l}\text { B8 Tunceli: Pülümür, between Bardakçı and } \\
\text { Derindere, } 300 \text { m from Kırdım junction to } \\
\text { Kırdım v. }\end{array}$ & 1750 & 22.07 .2014 & Forest & & $\begin{array}{l}\text { Euro.- } \\
\text { Sib. }\end{array}$ \\
\hline \multicolumn{8}{|l|}{ PLANTAGINACEAE Juss. } \\
\hline Anarrhinum orientale Benth. & 3746 & $\begin{array}{l}\text { B7 Tunceli: Center, } 1 \mathrm{~km} \text { from Kopuzlar to } \\
\text { Yolkonak }\end{array}$ & 1114 & 22.05 .2014 & Steppe & & Ir.-Tur. \\
\hline \multirow{2}{*}{$\begin{array}{l}\text { Chaenorhinum huber-morathii } \\
\text { P.H.Davis }\end{array}$} & 7459 & $\begin{array}{l}\text { B7 Tunceli: Pertek, between Pertek and } \\
\text { Mercimek v. }\end{array}$ & 1280 & 26.05 .2017 & \multirow{2}{*}{$\begin{array}{l}\text { Rocky } \\
\text { slopes }\end{array}$} & \multirow[t]{2}{*}{$*$} & \multirow{2}{*}{ Ir.-Tur. } \\
\hline & 7524 & $\begin{array}{l}\text { B7 Tunceli: Pertek, } 4 \mathrm{~km} \text { from Pertek to } \\
\text { Tunceli }\end{array}$ & 1385 & 03.06 .2017 & & & \\
\hline \multirow{2}{*}{$\begin{array}{l}\text { Kickxia spuria (L.) Dumort } \\
\text { subsp. integrifolia (Brot.) } \\
\text { R.Fernandes }\end{array}$} & 6054 & B7 Tunceli: Mazgirt, Yukarıyumca v. & 1430 & 15.08 .2014 & \multirow[b]{2}{*}{ Steppe } & & \\
\hline & 5737 & $\begin{array}{l}\text { B7 Tunceli: Mazgirt, } 3 \mathrm{~km} \text { from Akdüven to } \\
\text { Güleç }\end{array}$ & 1102 & 05.08 .2014 & & & Unk. \\
\hline & 6491 & $\begin{array}{l}\text { B7 Tunceli: Pülümür, } 1 \mathrm{~km} \text { from Turnadere } \\
\text { to Közlüce }\end{array}$ & 1435 & 29.05 .2015 & & & \\
\hline Linaria chalepensis (L.) Mill. & 6690 & $\begin{array}{l}\text { B7 Tunceli: Center, } 17 \mathrm{~km} \text { from Tunceli to } \\
\text { Ovacık (Munzur valley) }\end{array}$ & 1040 & 30.04 .2015 & Steppe & Rcd. & E.Medit. \\
\hline & 6503 & $\begin{array}{l}\text { B7 Tunceli: Center, } 1 \text { km from Sarıtaş to } \\
\text { Gözen (İksor), Kalan Valley }\end{array}$ & 1160 & 30.05 .2015 & & & \\
\hline Linaria corifolia Desf. & 5577 & $\begin{array}{l}\text { B7 Tunceli: Pülümür, } 3 \mathrm{~km} \text { from Erzincan- } \\
\text { Erzurum-Tunceli crossroads to Pülümür }\end{array}$ & 1335 & 22.07 .2014 & Steppe & $\begin{array}{l}* \\
\text { Rcd. }\end{array}$ & Ir.-Tur. \\
\hline Linaria kurdica Boiss. \& Hohen. & 6167 & $\begin{array}{l}\text { B7 Tunceli: } 10 \mathrm{~km} \text { from Pülümür - Erzincan } \\
\text { mainroad to Süleymanuşağı village }\end{array}$ & 2210 & 27.08 .2014 & & & \\
\hline $\begin{array}{l}\text { subsp. ararattca (1zvelev) } \\
\text { P.H.Davis }\end{array}$ & 5761 & $\begin{array}{l}\text { B7 Tunceli: Pülümür, } 45 \mathrm{~km} \text { from Tunceli to } \\
\text { Pülümür (Pülümür valley) }\end{array}$ & 1200 & 06.08 .2014 & Steppe & Rcd. & Ir.-Tur. \\
\hline Linaria kurdica Boiss. \& Hohen. & 5583 & B7 Tunceli: Pülümür, Dağyolu v. & 1725 & 22.07 .2014 & Steppe & Red. & Ir.-Tur. \\
\hline
\end{tabular}




\begin{tabular}{|c|c|c|c|c|c|c|c|}
\hline subsp. aucheri (Boiss.) P.H.Davis & & & & & & & \\
\hline \multirow{3}{*}{$\begin{array}{l}\text { Linaria kurdica Boiss. \& Hohen. } \\
\text { subsp. kurdica }\end{array}$} & 5419 & $\begin{array}{l}\text { B7 Tunceli: Hozat, } 700 \mathrm{~m} \mathrm{~N} \text { of Hozat- } \\
\text { Tunceli-Pertek crossroads }\end{array}$ & 1520 & 21.07.2014 & \multirow{3}{*}{ Steppe } & & \multirow{3}{*}{ Ir.-Tur. } \\
\hline & 5732 & B7 Tunceli: Mazgirt, $\mathrm{N}$ of Akdüven v. & 1130 & 05.08 .2014 & & & \\
\hline & 5392 & $\begin{array}{l}\text { B7 Tunceli: Nazımiye, } 3 \mathrm{~km} \text { from Dereova to } \\
\text { Yayıkağıl }\end{array}$ & 1630 & 20.07.2014 & & & \\
\hline Plantago major L. & 6024 & $\begin{array}{l}\text { B8 Tunceli: Pülümür, S of Ağaşenliği v., } \\
\text { between Ağaşenliği and Altınhüseyin }\end{array}$ & 1650 & 14.08.2014 & $\begin{array}{l}\text { Damp } \\
\text { places }\end{array}$ & & Unk. \\
\hline \multirow{6}{*}{$\begin{array}{l}\text { Veronica anagallis-aquatica } \mathrm{L} \text {. } \\
\text { subsp. lysimachioides (Boiss.) } \\
\text { M.A.Fisch. (三Veronica } \\
\text { lysimachioides Boiss.) }\end{array}$} & 5617 & B7 Tunceli: Nazımiye, $1 \mathrm{~km} \mathrm{~S}$ of Holik v. & 1910 & 23.07 .2014 & \multirow{6}{*}{$\begin{array}{l}\text { Riverside, } \\
\text { damp } \\
\text { places }\end{array}$} & & \multirow{6}{*}{ Unk. } \\
\hline & 4931 & $\begin{array}{l}\text { B7 Tunceli: Ovacık, } 8 \mathrm{~km} \text { from Eskigedik to } \\
\text { Işskvuran }\end{array}$ & 2120 & 19.06.2014 & & & \\
\hline & 5669 & $\begin{array}{l}\text { B7 Tunceli: Ovacık, } 9.5 \mathrm{~km} \text { from Ovacık to } \\
\text { Tunceli (Munzur Valley) }\end{array}$ & 1193 & 24.07.2014 & & & \\
\hline & $\mathbf{5 9 5 0}$ & B7 Tunceli: Ovacık, W of Ziyaret v. & 1335 & 08.08 .2014 & & & \\
\hline & 6077 & $\begin{array}{l}\text { B8 Tunceli: Pülümür, } 3 \mathrm{~km} \text { from Hasangazi } \\
\text { to Kırklar }\end{array}$ & 1890 & 16.08 .2014 & & & \\
\hline & 4860 & $\begin{array}{l}\text { B8 Tunceli: Pülümür, } 4 \mathrm{~km} \text { from Kırklar to } \\
\text { Kızılmescit }\end{array}$ & 2450 & 18.06.2014 & & & \\
\hline \multirow[b]{2}{*}{$\begin{array}{l}\text { Veronica anagalloides Guss. } \\
\text { subsp. heureka } \text { M.A.Fisch. }\end{array}$} & 5618 & B7 Tunceli: Nazımiye, 1 km S of Holik v. & 1910 & 23.07 .2014 & \multirow{2}{*}{$\begin{array}{l}\text { Riverside, } \\
\text { damp } \\
\text { places }\end{array}$} & \multirow[b]{2}{*}{ Rcd. } & \multirow[b]{2}{*}{ Ir.-Tur. } \\
\hline & 5993 & $\begin{array}{l}\text { B7 Tunceli: Pülümür, } 1.5 \mathrm{~km} \text { from Turnadere } \\
\text { to Közlüce }\end{array}$ & 1500 & 14.08.2014 & & & \\
\hline Veronica beccabunga $\mathrm{L}$. & 5511 & $\begin{array}{l}\text { B8 Tunceli: Pülümür, between Bardakçı to } \\
\text { Derindere }\end{array}$ & 1980 & 22.07.2014 & Riverside & & Unk. \\
\hline \multirow{2}{*}{ Veronica bozakmanii M.A.Fisch. } & 6424 & $\begin{array}{l}\text { B7 Tunceli: Pülümür, } 20 \mathrm{~km} \text { from Pülümür to } \\
\text { Tunceli (Pülümür valley) }\end{array}$ & 1215 & 12.04 .2015 & \multirow{2}{*}{ Steppe } & & \multirow{2}{*}{ Ir.-Tur. } \\
\hline & 4781 & $\begin{array}{l}\text { B7 Tunceli: Pülümür, } 2 \mathrm{~km} \text { from Turnadere } \\
\text { to Közlüce }\end{array}$ & 1560 & 18.06.2014 & & & \\
\hline $\begin{array}{l}\text { Veronica macrostachya } \text { Vahl } \\
\text { subsp. mardinensis (Bornm.) } \\
\text { M.A. Fisch. }\end{array}$ & 4170 & $\begin{array}{l}\text { B7 Tunceli: Nazımiye, } 5 \mathrm{~km} \text { from Nazımiye } \\
\text { to Tunceli }\end{array}$ & 1435 & 03.06.2014 & $\begin{array}{l}\text { Steppe, } \\
\text { degraded } \\
\text { oak forest }\end{array}$ & $*$ & Ir.-Tur. \\
\hline \multirow{2}{*}{$\begin{array}{l}\text { Veronica orientalis Mill. subsp. } \\
\text { nimrodi (Richt. ex Stapf) } \\
\text { M.A.Fisch. }\end{array}$} & 4246 & $\begin{array}{l}\text { B7 Tunceli: Ovacık, } 3 \text { km N of Işıkvuran v. } \\
\text { Munzur Mt. }\end{array}$ & 2020 & 04.06 .2014 & \multirow{2}{*}{ Steppe } & \multirow{2}{*}{$*$} & \multirow{2}{*}{ Unk. } \\
\hline & 4264 & $\begin{array}{l}\text { B7 Tunceli: Ovacık, } 3 \text { km N of Işıkvuran v. } \\
\text { Munzur Mt. }\end{array}$ & 2100 & 04.06 .2014 & & & \\
\hline \multirow{5}{*}{$\begin{array}{l}\text { Veronica orientalis Mill. subsp. } \\
\text { orientalis }\end{array}$} & 3698 & $\begin{array}{l}\text { B7 Tunceli: Center, Tunceli - Elazığ road, } \\
700 \text { m after Aktuluk v. }\end{array}$ & 930 & 22.05 .2014 & \multirow{5}{*}{$\begin{array}{l}\text { Steppe, } \\
\text { degraded } \\
\text { oak forest }\end{array}$} & & \multirow{5}{*}{ Ir.-Tur. } \\
\hline & 3790 & $\begin{array}{l}\text { B7 Tunceli: Center, between Tunceli and } \\
\text { Sütlüce, } 1.8 \mathrm{~km} \text { from Sütlüce junction }\end{array}$ & 1030 & 23.05 .2014 & & & \\
\hline & 3831 & $\begin{array}{l}\text { B7 Tunceli: Center, } 16 \mathrm{~km} \text { from Tunceli- } \\
\text { Pülümür-Sütlüce crossroads to Çıralı v. ( } 9 \mathrm{~km} \\
\text { before Çıralı v.) }\end{array}$ & 1850 & 23.05 .2014 & & & \\
\hline & 3966 & $\begin{array}{l}\text { B7 Tunceli: Center, } 2.5 \mathrm{~km} \text { from Tunceli- } \\
\text { Ovacık-Dedeağaç crossroads to Dedeağaç v. } \\
\text { (Munzur Valley) }\end{array}$ & 1100 & 25.05 .2014 & & & \\
\hline & 4105 & $\begin{array}{l}\text { B7 Tunceli: Mazgirt, } 7 \mathrm{~km} \text { from Kızılcık to } \\
\text { Göktepe }\end{array}$ & 935 & 02.06 .2014 & & & \\
\hline Veronica polita Fr. & 6430 & $\begin{array}{l}\text { B7 Tunceli: Center, } 7 \mathrm{~km} \text { from Tunceli to } \\
\text { Pülümür (Pülümür Valley) }\end{array}$ & 934 & 12.04 .2015 & Steppe & Rcd. & Unk. \\
\hline \multicolumn{8}{|l|}{ PLATANACEAE T.Lestib. } \\
\hline \multirow[t]{2}{*}{ Platanus orientalis L. } & 5082 & $\begin{array}{l}\text { B7 Tunceli: Çemişgezek, } 7 \mathrm{~km} \text { from } \\
\text { Payamdüzü to Bulgurtepe (Doğan Stream } \\
\text { Valley) }\end{array}$ & 922 & 21.06 .2014 & \multirow[t]{2}{*}{ Riverside } & & \multirow[t]{2}{*}{ Unk. } \\
\hline & 5138 & $\begin{array}{l}\text { B7 Tunceli: Çemişgezek, W of Bozağaç } \\
\text { village }\end{array}$ & 1120 & 28.06 .2014 & & & \\
\hline \multicolumn{8}{|l|}{ PLUMBAGINACEAE Juss. } \\
\hline Acantholimon calvertii Boiss. & 3899 & $\begin{array}{l}\text { B7 Tunceli: Pertek, } 9 \mathrm{~km} \text { from Pertek to } \\
\text { Cemişgezek, } 2.5 \mathrm{~km} \text { before Singeç bridge }\end{array}$ & 865 & 24.05 .2014 & Steppe & $*$ & Ir.-Tur. \\
\hline Plumbago europaea L. & 6135 & B7 Tunceli: Mazgirt, S of Dedebağ v. & 860 & 26.08 .2014 & Steppe & & Unk. \\
\hline \multicolumn{8}{|l|}{ POACEAE Barnhart } \\
\hline Aegilops cylindrica Host. & 4593 & $\begin{array}{l}\text { B7 Tunceli: Mazgirt, between Akdüven and } \\
\text { Koyunuşağ1 }\end{array}$ & 1125 & 16.06 .2014 & Steppe & Red. & Ir.-Tur. \\
\hline & 5081 & $\begin{array}{l}\text { B7 Tunceli: Pertek, between Dorutay and } \\
\text { Akdemir }\end{array}$ & 1300 & 21.06 .2014 & & & \\
\hline Aegilops neglecta Reg. ex Bertol. & 4405 & $\begin{array}{l}\text { B7 Tunceli: Pülümür, between Dereboyu and } \\
\text { Sağlamtaş }\end{array}$ & 1610 & 05.06 .2014 & Steppe & & Medit. \\
\hline $\begin{array}{l}\text { Aegilops speltoides Tausch var. } \\
\text { ligustica (Savign.) Bornm. }\end{array}$ & 5089 & $\begin{array}{l}\text { B7 Tunceli: Çemişgezek, between } \\
\text { Çemiş̧gezek and Elazığ, } 3 \mathrm{~km} \mathrm{NW} \text { of } \\
\text { Sarıbalta v. }\end{array}$ & 1050 & 21.06 .2014 & Steppe & & Unk. \\
\hline Aegilops triuncialis $\mathrm{L}$. & 4474 & B7 Tunceli: Hozat, 2 km NE of Akpınar v. & 1350 & 06.06 .2014 & Steppe & & Unk. \\
\hline Aegilops umbellulata Zhuk. & 4172 & $\begin{array}{l}\text { B7 Tunceli: Nazımiye, } 5 \mathrm{~km} \text { from Nazımiye } \\
\text { to Tunceli }\end{array}$ & 1435 & 03.06 .2014 & Steppe & & Ir.-Tur. \\
\hline Alopecurus aucheri Boiss. & 4919 & $\begin{array}{l}\text { B7 Tunceli: Ovacık, } 6 \mathrm{~km} \text { from Işıkvuran to } \\
\text { Eskigedik }\end{array}$ & 2250 & 19.06.2014 & Steppe & Red. & Ir.-Tur. \\
\hline
\end{tabular}




\begin{tabular}{|c|c|c|c|c|c|c|c|}
\hline \multirow[b]{2}{*}{ Avena barbata Pott ex Link } & 4535 & B7 Tunceli: Mazgirt, 1 km W of Kayacı v. & 985 & 16.06 .2014 & \multirow[b]{2}{*}{ Steppe } & \multirow[b]{2}{*}{ Rcd. } & \multirow[b]{2}{*}{ Medit. } \\
\hline & 4494 & $\begin{array}{l}\text { B7 Tunceli: Pertek, between Çemişgezek and } \\
\text { Elazığ, } 6 \mathrm{~km} \text { from Bulgurtepe to Payamdüzü }\end{array}$ & 980 & 06.06 .2014 & & & \\
\hline Briza humilis M.Bieb. & 4541 & B7 Tunceli: Mazgirt, 1 km W of Kayacı v. & 985 & 16.06 .2014 & Steppe & Rcd. & Unk. \\
\hline \multirow{2}{*}{ Bromus danthoniae Trin. } & 5050 & $\begin{array}{l}\text { B7 Tunceli: Ovacık, between Yazıören and } \\
\text { Gözeler }\end{array}$ & 1400 & 20.06 .2014 & \multirow{2}{*}{ Steppe } & & \multirow{2}{*}{ Unk. } \\
\hline & 4407 & $\begin{array}{l}\text { B7 Tunceli: Pülümür, between Dereboyu and } \\
\text { Sağlamtaş }\end{array}$ & 1610 & 05.06 .2014 & & & \\
\hline Bromus intermedius Guss. & 4376 & B7 Tunceli: Pülümür, Kocatepe v. & 1680 & 05.06 .2014 & Steppe & Red. & Unk. \\
\hline $\begin{array}{l}\text { Bromus japonicus Thunb. subsp. } \\
\text { anatolicus (Boiss. \& Heldr.) } \\
\text { Penzes }\end{array}$ & 4446 & $\begin{array}{l}\text { B7 Tunceli: Hozat, between Demirkapı and } \\
\text { Dervişcemal, } 300 \mathrm{~m} \text { to Buzlupınar junction }\end{array}$ & 1810 & 06.06 .2014 & Steppe & Red. & Unk. \\
\hline Bromus scoparius L. & 4747 & B7 Tunceli: Ovacık, $3 \mathrm{~km} \mathrm{~S}$ of Ovacık & 1315 & 17.06 .2014 & Steppe & Rcd. & Unk. \\
\hline Bromus squarrosus L. & 4998 & $\begin{array}{l}\text { B7 Tunceli: Ovacık, Ziyaret v., N of Munzur } \\
\text { Gözeleri }\end{array}$ & 1400 & 20.06 .2014 & Steppe & & Unk. \\
\hline \multirow{3}{*}{ Bromus tectorum $\mathrm{L}$. } & 4612 & $\begin{array}{l}\text { B7 Tunceli: Mazgirt, } 8 \mathrm{~km} \text { from Çukur to } \\
\text { Güleç }\end{array}$ & 1365 & 16.06 .2014 & \multirow{3}{*}{ Steppe } & & \multirow{3}{*}{ Unk. } \\
\hline & 4528 & B7 Tunceli: Mazgirt, 1 km W of Kayacı v. & 990 & 16.06 .2014 & & & \\
\hline & 4945 & $\begin{array}{l}\text { B7 Tunceli: Ovacık, N of Eskigedik v., } \\
\text { Munzur Mt. }\end{array}$ & 1915 & 19.06 .2014 & & & \\
\hline Chrysopogon gryllus (L.) Trin. & 5040 & $\begin{array}{l}\text { B7 Tunceli: Ovacık, between Yazıören and } \\
\text { Gözeler }\end{array}$ & 1400 & 20.06 .2014 & Steppe & & Unk. \\
\hline $\begin{array}{l}\text { Cynodon dactylon (L.) Pers. var. } \\
\text { villosus } \text { Regel }\end{array}$ & 4555 & $\begin{array}{l}\text { B7 Tunceli: Mazgirt, } 1 \mathrm{~km} \text { from Ortaharman } \\
\text { to Sülüntaş }\end{array}$ & 1220 & 16.06 .2014 & Steppe & & Unk. \\
\hline $\begin{array}{l}\text { Dactylis glomerata L. subsp. } \\
\text { glomerata }\end{array}$ & 4702 & $\begin{array}{l}\text { B7 Tunceli: Hozat, between Hozat and } \\
\text { Ovacık, around of Yüceldi v. }\end{array}$ & 1930 & 17.06 .2014 & Steppe & & $\begin{array}{l}\text { Euro.- } \\
\text { Sib. }\end{array}$ \\
\hline \multirow{2}{*}{$\begin{array}{l}\text { Dactylis glomerata L. subsp. } \\
\text { hispanica (Roth) Nyman }\end{array}$} & 4353 & $\begin{array}{l}\text { B7 Tunceli: Pülümür, } 2.5 \mathrm{~km} \text { from Kocatepe } \\
\text { to Sarıgül, near to Sarı̈ül junction }\end{array}$ & 1970 & 05.06 .2014 & \multirow{2}{*}{ Steppe } & & \multirow{2}{*}{ Unk. } \\
\hline & 4368 & $\begin{array}{l}\text { B7 Tunceli: Pülümür, } 3 \mathrm{~km} \text { from Sarıül to } \\
\text { Yalmanlar }\end{array}$ & 2250 & 05.06 .2014 & & & \\
\hline $\begin{array}{l}\text { Deschampsia caespitosa (L.) } \\
\text { P.Beauv. }\end{array}$ & 4870 & $\begin{array}{l}\text { B8 Tunceli: Pülümür, N of Kızılmescit v. (S } \\
\text { of Bağırpaşa Mt.) }\end{array}$ & 2080 & 18.06 .2014 & Steppe & & Unk. \\
\hline $\begin{array}{l}\text { Eremopoa persica (Trin.) } \\
\text { Roshev. }\end{array}$ & 4695 & $\begin{array}{l}\text { B7 Tunceli: Hozat, } 4.5 \mathrm{~km} \text { from Uzundal to } \\
\text { Yüceldi }\end{array}$ & 1970 & 17.06 .2014 & Steppe & & Ir.-Tur. \\
\hline $\begin{array}{l}\text { Festuca chalcophaea } \text { V.Krecz. \& } \\
\text { Bobrov. }\end{array}$ & 4728 & $\begin{array}{l}\text { B7 Tunceli: Hozat, } 15 \mathrm{~km} \text { from Ovacık to } \\
\text { Hozat (NE of Halitpınar v.) }\end{array}$ & 1800 & 17.06 .2014 & Steppe & Rcd. & Ir.-Tur. \\
\hline $\begin{array}{l}\text { Heteranthelium piliferum (Banks } \\
\text { \& Sol.) Hochst. }\end{array}$ & 4462 & $\begin{array}{l}\text { B7 Tunceli: Hozat, } 3 \mathrm{~km} \text { from Dervişcemal } \\
\text { to Balkaynar }\end{array}$ & 1675 & 06.06 .2014 & Steppe & & Ir.-Tur. \\
\hline \multirow{6}{*}{ Hordeum bulbosum L. } & 5036 & $\begin{array}{l}\text { B7 Tunceli: Ovacık, } 3 \mathrm{~km} \text { from Cevizlidere } \\
\text { to Kozluca, SE of Karataş v. }\end{array}$ & 1625 & 20.06 .2014 & \multirow{6}{*}{ Steppe } & & \multirow{6}{*}{ Unk. } \\
\hline & 5008 & $\begin{array}{l}\text { B7 Tunceli: Ovacık, } 5 \mathrm{~km} \text { from Eğripınar to } \\
\text { Yenikonak }\end{array}$ & 1480 & 20.06 .2014 & & & \\
\hline & 4233 & $\begin{array}{l}\text { B7 Tunceli: Ovacık, } 15 \mathrm{~km} \text { from Ovacık- } \\
\text { Tunceli-Iş1kvuran crossroads to Işıkvuran }\end{array}$ & 1670 & 04.06 .2014 & & & \\
\hline & 4396 & $\begin{array}{l}\text { B7 Tunceli: Pülümür, } 5 \text { km from Çakırkaya } \\
\text { and Dereboyu }\end{array}$ & 1870 & 05.06 .2014 & & & \\
\hline & 4377 & B7 Tunceli: Pülümür, Kocatepe v. & 1680 & 05.06 .2014 & & & \\
\hline & 3945 & $\begin{array}{l}\text { B7 Tunceli: Center, } 5 \mathrm{~km} \text { from Tunceli to } \\
\text { Ovacık (Munzur Valley) }\end{array}$ & 970 & 25.05 .2014 & & & \\
\hline Melica ciliata $\mathrm{L}$. & 4427 & $\begin{array}{l}\text { B7 Tunceli: Pülümür, } 9 \mathrm{~km} \text { from Pülümür to } \\
\text { Tunceli (Pülümür valley), S of Kangallı v. }\end{array}$ & 1324 & 05.06 .2014 & Steppe & & Unk. \\
\hline \multirow{2}{*}{$\begin{array}{l}\text { Melica persica Kunth subsp. } \\
\text { inaequiglumis (Boiss.) Bor }\end{array}$} & 4953 & $\begin{array}{l}\text { B7 Tunceli: Ovacık, N of Eskigedik v., } \\
\text { Munzur Mt. }\end{array}$ & 2160 & 19.06 .2014 & \multirow[t]{2}{*}{ Steppe } & & \multirow[t]{2}{*}{ Unk. } \\
\hline & 4979 & B7 Tunceli: Center, Gözen (İksor) v. & 1490 & 19.06 .2014 & & & \\
\hline \multirow{2}{*}{ Phleum pratense $\mathrm{L}$. } & 4447 & $\begin{array}{l}\text { B7 Tunceli: Hozat, between Demirkapı and } \\
\text { Dervișcemal, } 300 \mathrm{~m} \text { to Buzlupınar junction }\end{array}$ & 1810 & 06.06 .2014 & \multirow{2}{*}{ Steppe } & \multirow{2}{*}{ Rcd. } & \multirow{2}{*}{$\begin{array}{l}\text { Euro.- } \\
\text { Sib. }\end{array}$} \\
\hline & 4928 & $\begin{array}{l}\text { B7 Tunceli: Ovacık, } 8 \mathrm{~km} \text { from Eskigedik to } \\
\text { Işıkvuran }\end{array}$ & 2120 & 19.06 .2014 & & & \\
\hline \multirow{2}{*}{$\begin{array}{l}\text { Phragmites australis (Cav.) Trin. } \\
\text { ex Steud. }\end{array}$} & $6056 a$ & B7 Tunceli: Mazgirt, Yukarıyumca v. & 1430 & 15.08 .2014 & \multirow[b]{2}{*}{ Riverside } & & \\
\hline & 6076 & $\begin{array}{l}\text { B8 Tunceli: Pülümür, } 3 \mathrm{~km} \text { from Hasangazi } \\
\text { to Kirklar }\end{array}$ & 1890 & 16.08 .2014 & & & Unk. \\
\hline Poa bulbosa L. & 4350 & $\begin{array}{l}\text { B7 Tunceli: Pülümür, } 2.5 \mathrm{~km} \text { from Kocatepe } \\
\text { to Sarıgül, near to Sarı̈ül junction }\end{array}$ & 1970 & 05.06 .2014 & Steppe & & Unk. \\
\hline $\begin{array}{l}\text { Psathyrostachys fragilis (Boiss.) } \\
\text { Nevski subsp. villosa } \text { C.Baden }\end{array}$ & 4922 & $\begin{array}{l}\text { B7 Tunceli: Ovacık, } 7 \mathrm{~km} \text { from Işıkvuran to } \\
\text { Eskigedik }\end{array}$ & 2170 & 19.06.2014 & Steppe & & Ir.-Tur. \\
\hline $\begin{array}{l}\text { Setaria verticillata (L.) P.Beauv. } \\
\text { var. ambigua (Guss.) Parl. }\end{array}$ & 4481 & $\begin{array}{l}\text { B7 Tunceli: Hozat, Çemişgezek-Elazığ road, } \\
\text { S of Akpınar v. }\end{array}$ & 1224 & 06.06 .2014 & Steppe & Rcd. & Unk. \\
\hline Sorghum halepense (L.) Pers. & 4503 & $\begin{array}{l}\text { B7 Tunceli: Çemişgezek, } 5 \mathrm{~km} \text { from } \\
\text { Payamdüzü to Yemişdere }\end{array}$ & 865 & 06.06 .2014 & Steppe & & Unk. \\
\hline $\begin{array}{l}\text { Stipa ehrenbergiana Trin. \& } \\
\text { Rupr. }\end{array}$ & 4354 & $\begin{array}{l}\text { B7 Tunceli: Pülümür, } 2.5 \mathrm{~km} \text { from Kocatepe } \\
\text { to Sarıgül, near to Sarıgül junction }\end{array}$ & 1970 & 05.06 .2014 & Steppe & & Ir.-Tur. \\
\hline $\begin{array}{l}\text { Taeniatherum caput-medusae } \\
\text { (L.) Nevski subsp. crinitum }\end{array}$ & 4464 & $\begin{array}{l}\text { B7 Tunceli: Hozat, } 3 \mathrm{~km} \text { from Dervişcemal } \\
\text { to Balkaynar }\end{array}$ & 1675 & 06.06 .2014 & Steppe & & Ir.-Tur. \\
\hline (Schreb.) Melderis & 4469 & B7 Tunceli: Hozat, $3.5 \mathrm{~km}$ from Hozat to & 1470 & 06.06 .2014 & & & \\
\hline
\end{tabular}




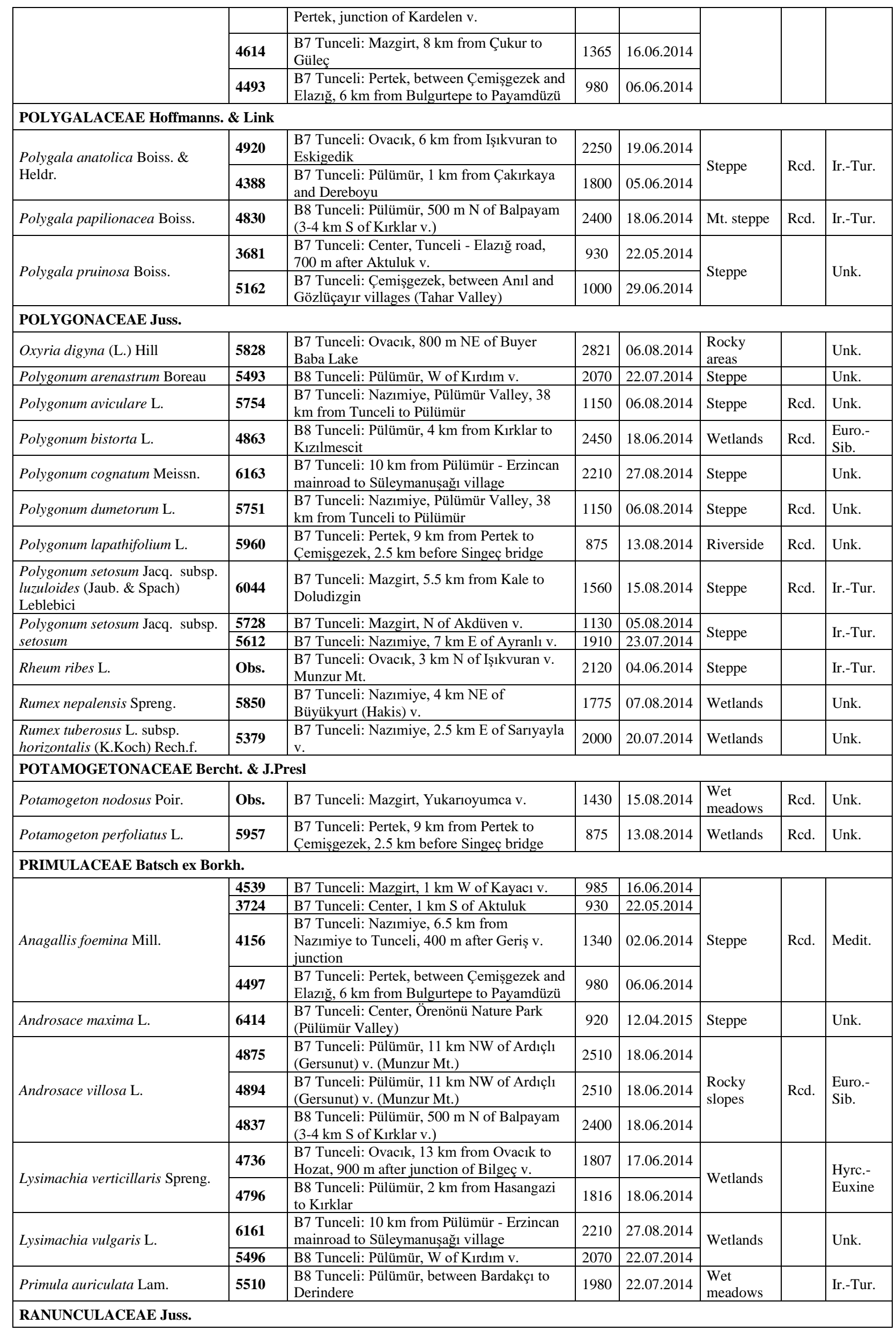




\begin{tabular}{|c|c|c|c|c|c|c|c|}
\hline $\begin{array}{l}\text { Adonis aestivalis L. subsp. } \\
\text { parviflora (Fisch. ex DC.) } \\
\text { E.A.Busch }\end{array}$ & 4423 & $\begin{array}{l}\text { B7 Tunceli: Pülümür, } 9 \mathrm{~km} \text { from Pülümür to } \\
\text { Tunceli (Pülümür valley), S of Kangallı v. }\end{array}$ & 1324 & 05.06 .2014 & Steppe & & Unk. \\
\hline $\begin{array}{l}\text { Adonis cyllenea Boiss., Heldr. \& } \\
\text { Orph. var. paryadrica Boiss. }\end{array}$ & Obs. & $\begin{array}{l}\text { B7 Tunceli: Pülümür, between Sarıgül and } \\
\text { Kocatepe, Buyer waterfall }\end{array}$ & 2150 & 30.05 .2017 & Mt. steppe & Rcd. & Unk. \\
\hline Adonis eriocalycina Boiss. & 3791 & $\begin{array}{l}\text { B7 Tunceli: Center, between Tunceli and } \\
\text { Sütlüce, } 1.8 \mathrm{~km} \text { from Sütlüce junction }\end{array}$ & 1030 & 23.05 .2014 & Steppe & & Unk. \\
\hline Anemone coronaria $\mathrm{L}$. & 4279 & $\begin{array}{l}\text { B7 Tunceli: Ovacık, } 3 \text { km N of Işıkvuran v. } \\
\text { Munzur Mt. }\end{array}$ & 2150 & 04.06 .2014 & Mt. steppe & Rcd. & Medit. \\
\hline Caltha palustris $\mathrm{L}$. & 6092 & B8 Tunceli: Pülümür, 2.6 km E of Kırklar v. & 2120 & 16.08 .2014 & $\begin{array}{l}\text { Wet } \\
\text { meadows }\end{array}$ & & Unk. \\
\hline \multirow{3}{*}{ Clematis orientalis $\mathrm{L}}$. & 6064 & $\begin{array}{l}\text { B7 Tunceli: Center, } 4 \mathrm{~km} \text { from Tunceli center } \\
\text { to Babaocağ1 }\end{array}$ & 930 & 15.08 .2014 & \multirow{3}{*}{ Oak forest } & \multirow{3}{*}{ Rcd. } & \multirow{3}{*}{ Unk. } \\
\hline & 5574 & $\begin{array}{l}\text { B7 Tunceli: Pülümür, } 2 \mathrm{~km} \text { from Erzincan- } \\
\text { Erzurum-Tunceli crossroads to Pülümür }\end{array}$ & 1260 & 22.07.2014 & & & \\
\hline & 5690 & $\begin{array}{l}\text { B7 Tunceli: Center, } 21 \mathrm{~km} \text { from Tunceli to } \\
\text { Ovacık (Munzur Valley) }\end{array}$ & 1024 & 24.07.2014 & & & \\
\hline $\begin{array}{l}\text { Consolida glandulosa (Boiss. \& } \\
\text { Huet) Bornm. }\end{array}$ & 5576 & $\begin{array}{l}\text { B7 Tunceli: Pülümür, } 3 \mathrm{~km} \text { from Erzincan- } \\
\text { Erzurum-Tunceli crossroads to Pülümür }\end{array}$ & 1335 & 22.07 .2014 & Steppe & $*$ & Ir.-Tur. \\
\hline $\begin{array}{l}\text { Consolida oliveriana (DC.) } \\
\text { Schödinger }\end{array}$ & 5739 & $\begin{array}{l}\text { B7 Tunceli: Mazgirt, } 3 \text { km from Akdüven to } \\
\text { Güleç }\end{array}$ & 1102 & 05.08 .2014 & Steppe & & Ir.-Tur. \\
\hline \multirow{2}{*}{$\begin{array}{l}\text { Consolida scleroclada (Boiss.) } \\
\text { Schrödinger var. rigida (Freyn \& } \\
\text { Sint.) P.H.Davis }\end{array}$} & 5978 & $\begin{array}{l}\text { B7 Tunceli: Hozat, } 2.5 \mathrm{~km} \text { from Hozat to } \\
\text { Dervişcemal v. }\end{array}$ & 1523 & 13.08.2014 & \multirow{2}{*}{ Steppe } & & \multirow{2}{*}{ Ir.-Tur. } \\
\hline & 5301 & $\begin{array}{l}\text { B7 Tunceli: Center, } 14 \mathrm{~km} \text { from Tunceli to } \\
\text { Pülümür (Pülümür Valley) }\end{array}$ & 970 & 20.07.2014 & & & \\
\hline Delphinium albiflorum DC. & 5642 & $\begin{array}{l}\text { B7 Tunceli: Ovacık, } 30 \mathrm{~km} \text { from Tunceli to } \\
\text { Ovacık (Munzur Valley) }\end{array}$ & 1070 & 24.07.2014 & Steppe & & Unk. \\
\hline \multirow{3}{*}{$\begin{array}{l}\text { Delphinium kurdicum Boiss. \& } \\
\text { Hohen. }\end{array}$} & 5900 & $\begin{array}{l}\text { B7 Tunceli: Ovacık, Tunceli-Ovacık- } \\
\text { Yakatarla junction }\end{array}$ & 1240 & 08.08 .2014 & \multirow{3}{*}{ Steppe } & & \multirow{3}{*}{ Ir.-Tur. } \\
\hline & 5893 & $\begin{array}{l}\text { B7 Tunceli: Pülümür, } 42 \mathrm{~km} \text { from Tunceli to } \\
\text { Pülümür (Pülümür Valley), across of } \\
\text { Ağlayan Kayalar }\end{array}$ & 1570 & 07.08 .2014 & & & \\
\hline & 5488 & $\begin{array}{l}\text { B8 Tunceli: Pülümür, between Derindere and } \\
\text { Kırdım }\end{array}$ & 2045 & 22.07.2014 & & & \\
\hline \multirow[b]{2}{*}{ Delphinium peregrinum L. } & 6118 & B7 Tunceli: Pertek, 2 km W of Bulgurtepe v. & 1060 & 17.08 .2014 & \multirow{2}{*}{$\begin{array}{l}\text { Degraded } \\
\text { oak forest, } \\
\text { steppe }\end{array}$} & & \multirow[b]{2}{*}{ Medit. } \\
\hline & 5968 & $\begin{array}{l}\text { B7 Tunceli: Pertek, } 9 \mathrm{~km} \text { from Pertek to } \\
\text { Çemişgezek, } 2.5 \mathrm{~km} \text { before Singeç bridge }\end{array}$ & 875 & 13.08.2014 & & & \\
\hline \multirow{2}{*}{ Eranthis hyemalis (L.) Salisb. } & 4242 & $\begin{array}{l}\text { B7 Tunceli: Ovacık, } 3 \text { km N of Işıkvuran v. } \\
\text { Munzur Mt. }\end{array}$ & 2020 & 04.06 .2014 & \multirow{2}{*}{$\begin{array}{l}\text { Wet } \\
\text { meadows }\end{array}$} & & \multirow{2}{*}{ Unk. } \\
\hline & 6405 & $\begin{array}{l}\text { B7 Tunceli: Ovacık, } 1.5 \mathrm{~km} \text { E of Ovacık } \\
\text { center }\end{array}$ & 1230 & 10.04.2015 & & & \\
\hline $\begin{array}{l}\text { Nigella arvensis L. var. glauca } \\
\text { Boiss. }\end{array}$ & 3902 & $\begin{array}{l}\text { B7 Tunceli: Pertek, } 12 \mathrm{~km} \text { from Pertek to } \\
\text { Çemişgezek, around of Singeç bridge }\end{array}$ & 855 & 24.05 .2014 & Steppe & & Unk. \\
\hline Nigella latisecta P.H.Davis & 3751 & $\begin{array}{l}\text { B7 Tunceli: Center, } 1 \mathrm{~km} \text { from Kopuzlar to } \\
\text { Yolkonak }\end{array}$ & 1114 & 22.05 .2014 & Steppe & & Ir.-Tur. \\
\hline Nigella nigellastrum (L.) Willk. & 4525 & B7 Tunceli: Mazgirt, 1 km W of Kayacı v. & 990 & 16.06 .2014 & Steppe & Rcd. & Unk. \\
\hline Nigella oxypetala Boiss. & 4114 & $\begin{array}{l}\text { B7 Tunceli: Mazgirt, between Güneşdere and } \\
\text { Kızılcık }\end{array}$ & 1090 & 02.06 .2014 & Steppe & & Ir.-Tur. \\
\hline $\begin{array}{l}\text { Pulsatilla violacea } \text { Rupr. subsp. } \\
\text { armena (Boiss.) Luferov }\end{array}$ & 6675 & $\begin{array}{l}\text { B7 Tunceli: Pülümür, } 5 \mathrm{~km} \text { from Sarıgül to } \\
\text { Yalmanlar }\end{array}$ & 2721 & 03.06 .2015 & Steppe & Rcd. & Unk. \\
\hline Ranunculus arvensis $\mathrm{L}$. & 3723 & B7 Tunceli: Center, $1 \mathrm{~km} \mathrm{~S}$ of Aktuluk & 930 & 22.05 .2014 & Steppe & & Unk. \\
\hline Ranunculus fenzlii Boiss. & 4744 & $\begin{array}{l}\text { B7 Tunceli: Ovacık, } 10 \mathrm{~km} \text { from Ovacık to } \\
\text { Hozat, in junction of Kuşluca v. }\end{array}$ & 1710 & 17.06.2014 & Steppe & $*$ & Ir.-Tur. \\
\hline $\begin{array}{l}\text { Ranunculus munzurensis } \text { S.Erik } \\
\& \text { Yild. }\end{array}$ & 6540 & $\begin{array}{l}\text { B7 Tunceli: Ovacık, } 7 \mathrm{~km} \text { from Yoncalı to } \\
\text { Ağaçpınar }\end{array}$ & 2015 & 01.06 .2015 & On rocks & $*$ & Ir.-Tur. \\
\hline Ranunculus repens $\mathrm{L}$. & 3716 & $\begin{array}{l}\text { B7 Tunceli: Center, between Tunceli and } \\
\text { Pertek, junction of Kopuzlar v. }\end{array}$ & 1020 & 22.05 .2014 & Steppe & & Unk. \\
\hline \multirow[b]{2}{*}{ Thalictrum isopyroides C.A.Mey. } & 4371 & B7 Tunceli: Pülümür, Kocatepe v. & 1680 & 05.06 .2014 & \multirow{2}{*}{$\begin{array}{l}\text { Rock } \\
\text { crevices, } \\
\text { steppe } \\
\end{array}$} & & \multirow[b]{2}{*}{ Ir.-Tur. } \\
\hline & 6509 & $\begin{array}{l}\text { B7 Tunceli: Center, } 4 \text { km from Sarıtaş to } \\
\text { Gözen (İksor), Kalan Valley }\end{array}$ & 1350 & 30.05 .2015 & & & \\
\hline \multicolumn{8}{|l|}{ RHAMNACEAE Juss. } \\
\hline \multirow{2}{*}{ Paliurus spina-christi P.Mill. } & 4487 & $\begin{array}{l}\text { B7 Tunceli: Çemişgezek, } 2 \mathrm{~km} \text { from } \\
\text { Bulgurtepe to Payamdüzü }\end{array}$ & 1060 & 06.06 .2014 & \multirow{2}{*}{$\begin{array}{l}\text { Steppe, in } \\
\text { gardens }\end{array}$} & & \multirow{2}{*}{ Unk. } \\
\hline & 3711 & $\begin{array}{l}\text { B7 Tunceli: Center, between Tunceli and } \\
\text { Pertek, junction of Kopuzlar v. }\end{array}$ & 1020 & 22.05 .2014 & & & \\
\hline \multicolumn{8}{|l|}{ ROSACEAE Juss. } \\
\hline Agrimonia repens $\mathrm{L}$. & 5033 & B7 Tunceli: Ovacık, 2 km N of Yenikonak v. & 1705 & 20.06.2014 & $\begin{array}{l}\text { Wet } \\
\text { meadows }\end{array}$ & & Unk. \\
\hline Alchemilla holocycla Rothm. & 5366 & B7 Tunceli: Nazımiye, 2 km E of Sarıyayla v. & 1945 & 20.07 .2014 & $\begin{array}{l}\text { Wet } \\
\text { meadows }\end{array}$ & $*$ & Ir.-Tur. \\
\hline Amelanchier ovalis Medik. & 4893 & $\begin{array}{l}\text { B7 Tunceli: Pülümür, } 11 \text { km NW of Ardıçlı } \\
\text { (Gersunut) v. (Munzur Mt.) }\end{array}$ & 2510 & 18.06 .2014 & $\begin{array}{l}\text { Rocky } \\
\text { slopes }\end{array}$ & & Unk. \\
\hline Amygdalus communis L. & 5286 & $\begin{array}{l}\text { B7 Tunceli: Pertek, } 8 \mathrm{~km} \text { from Pertek to } \\
\text { Tunceli }\end{array}$ & 1300 & 19.07.2014 & $\begin{array}{l}\text { Steppe, in } \\
\text { gardens }\end{array}$ & & Unk. \\
\hline
\end{tabular}




\begin{tabular}{|c|c|c|c|c|c|c|c|}
\hline $\begin{array}{l}\text { Amygdalus trichamygdalus } \\
\text { (Hand.-Mazz.) Woronow }\end{array}$ & 3910 & $\begin{array}{l}\text { B7 Tunceli: Pertek, } 12 \mathrm{~km} \text { from Pertek to } \\
\text { Çemişgezek, around of Singeç bridge }\end{array}$ & 855 & 24.05 .2014 & Steppe & Rcd. & Ir.-Tur. \\
\hline \multirow{2}{*}{ Cerasus mahaleb (L.) Mill. } & 6256 & $\begin{array}{l}\text { B7 Tunceli: Center, between Tüllük and } \\
\text { Dedeağaç, N of Tüllük }\end{array}$ & 1750 & 05.09 .2014 & \multirow{2}{*}{$\begin{array}{l}\text { Steppe, } \\
\text { dried river } \\
\text { bad }\end{array}$} & & \multirow{2}{*}{ Unk. } \\
\hline & 5920 & $\begin{array}{l}\text { B7 Tunceli: Ovacık, } 3 \mathrm{~km} \mathrm{~N} \text { of Işıkvuran v. } \\
\text { Munzur Mt. }\end{array}$ & 2005 & 08.08 .2014 & & & \\
\hline $\begin{array}{l}\text { Cerasus microcarpa (C.A.Mey.) } \\
\text { Boiss. }\end{array}$ & 5997 & $\begin{array}{l}\text { B7 Tunceli: Pülümür, } 1.5 \mathrm{~km} \text { from Turnadere } \\
\text { to Közlüce }\end{array}$ & 1500 & 14.08 .2014 & \begin{tabular}{|l|l|}
$\begin{array}{l}\text { Stream } \\
\text { side }\end{array}$ \\
\end{tabular} & & Ir.-Tur. \\
\hline \multirow{2}{*}{$\begin{array}{l}\text { Cotoneaster nummularius Fisch. } \\
\& \text { C.A.Mey. }\end{array}$} & 5838 & $\begin{array}{l}\text { B7 Tunceli: Nazımiye, } 400 \mathrm{~m} \text { from } \\
\text { Aşağırabat bridge to Büyükyurt (Hakis) v. }\end{array}$ & 1054 & 07.08 .2014 & \multirow{2}{*}{$\begin{array}{l}\text { Riverside, } \\
\text { forest }\end{array}$} & & \multirow{2}{*}{ Unk. } \\
\hline & 6244 & $\begin{array}{l}\text { B7 Tunceli: Nazımiye, } 400 \mathrm{~m} \text { from } \\
\text { Așağırabat bridge to Büyükyurt (Hakis) v. }\end{array}$ & 1070 & 05.09 .2014 & & & \\
\hline Crataegus monogyna Jacq. & 6254 & $\begin{array}{l}\text { B7 Tunceli: Nazımiye, Pülümür Valley, } 31 \\
\text { km from Tunceli to Pülümür }\end{array}$ & 1085 & 05.09.2014 & Steppe & & Unk. \\
\hline \multirow{4}{*}{$\begin{array}{l}\text { Crataegus orientalis Pall. ex } \\
\text { M.Bieb. subsp. orientalis }\end{array}$} & 6123 & $\begin{array}{l}\text { B7 Tunceli: Çemişgezek, between Gülbahçe } \\
\text { and Dedebeyli }\end{array}$ & 1277 & 17.08 .2014 & \multirow{4}{*}{ Steppe } & & \multirow{4}{*}{ Unk. } \\
\hline & 5265 & B7 Tunceli: Pertek, Camikebir nh. & 1100 & 19.07.2014 & & & \\
\hline & 6012 & $\begin{array}{l}\text { B8 Tunceli: Pülümür, } 4 \text { km from Hasangazi } \\
\text { to Balpayam }\end{array}$ & 1832 & 14.08 .2014 & & & \\
\hline & 5548 & $\begin{array}{l}\text { B8 Tunceli: Pülümür, between Pülümür and } \\
\text { Yedisu, } 10 \mathrm{~km} \text { after Derindere v. }\end{array}$ & 1717 & 22.07 .2014 & & & \\
\hline \multirow{2}{*}{$\begin{array}{l}\text { Crataegus orientalis Pall. ex } \\
\text { M.Bieb. subsp. szovitsii (Pojark.) } \\
\text { K.I.Chr. }\end{array}$} & 6139 & B7 Tunceli: Mazgirt, S of Dedebağ v. & 860 & 26.08 .2014 & \multirow[b]{2}{*}{$\begin{array}{l}\text { Riverside, } \\
\text { steppe }\end{array}$} & & \multirow[b]{2}{*}{ Ir.-Tur. } \\
\hline & 5378 & $\begin{array}{l}\text { B7 Tunceli: Nazımiye, } 2.5 \text { km E of Sarıyayla } \\
\text { v. }\end{array}$ & 2000 & 20.07.2014 & & & \\
\hline Filipendula ulmaria (L.) Maxim. & 5626 & B7 Tunceli: Nazımiye, 1 km S of Holik v. & 1990 & 23.07.2014 & \begin{tabular}{|l|} 
Wet \\
meadows
\end{tabular} & & \begin{tabular}{|l|} 
Euro.- \\
Sib. \\
\end{tabular} \\
\hline Geum heterocarpum Boiss. & 6541 & $\begin{array}{l}\text { B7 Tunceli: Ovacık, } 7 \mathrm{~km} \text { from Yoncalı to } \\
\text { Ağaçpınar }\end{array}$ & 2015 & 01.06 .2015 & \begin{tabular}{|l|l|} 
Rocky \\
areas
\end{tabular} & Rcd. & Unk. \\
\hline Geum rivale $\mathrm{L}$. & 4867 & $\begin{array}{l}\text { B8 Tunceli: Pülümür, } 4 \text { km from Kırklar to } \\
\text { Kızılmescit }\end{array}$ & 2450 & 18.06 .2014 & $\begin{array}{l}\text { Damp } \\
\text { places }\end{array}$ & & Unk. \\
\hline Geum urbanum $\mathrm{L}$. & 3970 & $\begin{array}{l}\text { B7 Tunceli: Center, } 2.5 \mathrm{~km} \text { from Tunceli- } \\
\text { Ovacik-Dedeağaç crossroads to Dedeağaç v. } \\
\text { (Munzur Valley) }\end{array}$ & 1100 & 25.05 .2014 & $\begin{array}{l}\text { Damp } \\
\text { places }\end{array}$ & & $\begin{array}{l}\text { Euro.- } \\
\text { Sib. }\end{array}$ \\
\hline \multirow{3}{*}{$\begin{array}{l}\text { Malus sylvestris (L.) Mill. subsp. } \\
\text { orientalis (Uglitzk.) Browicz }\end{array}$} & 6026 & $\begin{array}{l}\text { B8 Tunceli: Pülümür, S of Ağaşenliği v., } \\
\text { between Ağaşenliği and Altınhüseyin }\end{array}$ & 1650 & 14.08.2014 & \multirow{3}{*}{ Steppe } & & \multirow{3}{*}{ Unk. } \\
\hline & 6107 & $\begin{array}{l}\text { B7 Tunceli: Pülümür, E of Ardıçlı (Gersunut) } \\
\text { v. }\end{array}$ & 1767 & 16.08 .2014 & & & \\
\hline & 5518 & $\begin{array}{l}\text { B8 Tunceli: Pülümür, between Bardakçı to } \\
\text { Derindere }\end{array}$ & 1980 & 22.07 .2014 & & & \\
\hline Potentilla argentea $\mathrm{L}$. & 5625 & B7 Tunceli: Nazımiye, 1 km S of Holik v. & 1990 & 23.07 .2014 & \begin{tabular}{|l|} 
Rock \\
crevices
\end{tabular} & & Unk. \\
\hline Potentilla inclinata Vill. & 6160 & $\begin{array}{l}\text { B7 Tunceli: } 10 \mathrm{~km} \text { from Pülümür - Erzincan } \\
\text { mainroad to Süleymanuşağı village }\end{array}$ & 2210 & 27.08 .2014 & Steppe & Rcd. & Unk. \\
\hline Potentilla recta $\mathrm{L}$. & 5538 & $\begin{array}{l}\text { B8 Tunceli: Pülümür, 1-2 km E of Bağırpaşa } \\
\text { Mt., } 2 \mathrm{~km} \mathrm{~N} \text { of Şampaşakaraderbent v. }\end{array}$ & 1886 & 22.07 .2014 & Steppe & & Unk. \\
\hline \multirow[t]{2}{*}{ Potentilla reptans $\mathrm{L}$. } & 5662 & $\begin{array}{l}\text { B7 Tunceli: Ovacık, } 10 \mathrm{~km} \text { from Eğripınar to } \\
\text { Yenikonak }\end{array}$ & 1650 & 24.07 .2014 & \multirow[t]{2}{*}{ Steppe } & & \multirow[t]{2}{*}{ Unk. } \\
\hline & 6085 & B8 Tunceli: Pülümür, SE of Kırklar v. & 2015 & 16.08 .2014 & & & \\
\hline Potentilla speciosa Willd. & 5807 & $\begin{array}{l}\text { B7 Tunceli: Ovacik, } 8 \mathrm{~km} \text { from Sarigül to } \\
\text { Yalmanlar, the walk way of Buyer Baba } \\
\text { sanctuary }\end{array}$ & 2680 & 06.08 .2014 & Steppe & & Unk. \\
\hline \multirow{3}{*}{ Prunus divaricata Ledeb. } & 6206 & $\begin{array}{l}\text { B7 Tunceli: Hozat, } 18 \mathrm{~km} \text { from Hozat to } \\
\text { Ovacik }\end{array}$ & 1967 & 28.08 .2014 & \multirow{3}{*}{ Oak forest } & & \multirow{3}{*}{ Unk. } \\
\hline & 5879 & $\begin{array}{l}\text { B7 Tunceli: Nazımiye, } 6 \mathrm{~km} \mathrm{NE} \text { of } \\
\text { Büyükyurt (Hakis) v. }\end{array}$ & 2050 & 07.08 .2014 & & & \\
\hline & 6035 & $\begin{array}{l}\text { B8 Tunceli: Pülümür, } 2 \mathrm{~km} \mathrm{~W} \text { of } \\
\text { Altınhüseyin }\end{array}$ & 1905 & 14.08 .2014 & & & \\
\hline $\begin{array}{l}\text { Pyrus elaeagnifolia Pall. subsp. } \\
\text { elaeagnifolia }\end{array}$ & 6007 & $\begin{array}{l}\text { B7 Tunceli: Pülümür, between Közlüce and } \\
\text { Hacılı }\end{array}$ & 1576 & 14.08 .2014 & Steppe & & Unk. \\
\hline $\begin{array}{l}\text { Pyrus elaeagnifolia Pall. subsp. } \\
\text { kotschyana (Boiss.) Browicz }\end{array}$ & 6025 & $\begin{array}{l}\text { B8 Tunceli: Pülümür, S of Ağaşenliği v., } \\
\text { between Ağaşenliği and Altı̈nuseyin }\end{array}$ & 1650 & 14.08 .2014 & Steppe & & Unk. \\
\hline \multirow{6}{*}{ Rosa canina $\mathrm{L}$. } & 6063 & B7 Tunceli: Center, S of Babaocağ v. & 1117 & \begin{tabular}{|l|}
15.08 .2014 \\
\end{tabular} & \multirow{6}{*}{$\begin{array}{l}\text { Steppe, in } \\
\text { gardens }\end{array}$} & & \multirow{6}{*}{ Unk. } \\
\hline & 6230 & $\begin{array}{l}\text { B7 Tunceli: Center, } 6 \mathrm{~km} \text { from Tunceli to } \\
\text { Pülümür (Pülümür Valley) }\end{array}$ & 934 & 05.09 .2014 & & & \\
\hline & 5701 & $\begin{array}{l}\text { B7 Tunceli: Pertek, between Pirinçci and } \\
\text { Karagüney }\end{array}$ & 1153 & 25.07 .2014 & & & \\
\hline & 6098 & $\begin{array}{l}\text { B7 Tunceli: Pülümür, } 3 \mathrm{~km} \text { from Pülümür to } \\
\text { Hiver }\end{array}$ & 1917 & 16.08 .2014 & & & \\
\hline & 5499 & B8 Tunceli: Pülümür, W of Kırdım v. & 2070 & \begin{tabular}{|l|}
22.07 .2014 \\
\end{tabular} & & & \\
\hline & 6148 & $\begin{array}{l}\text { B7 Tunceli: Pülümür, } 2 \mathrm{~km} \text { from Erzincan- } \\
\text { Pülümür-Süleymanuşağı to Süleymanyuşağ }\end{array}$ & 1683 & 27.08 .2014 & & & \\
\hline Rosa mollis Sm. & 5500 & B8 Tunceli: Pülümür, SW of Kırdım v. & 2070 & 22.07 .2014 & Steppe & & Unk. \\
\hline Rosa orientalis A.Dupont ex DC. & 5921 & $\begin{array}{l}\text { B7 Tunceli: Ovacık, } 3 \text { km N of Işıkvuran v. } \\
\text { Munzur Mt. }\end{array}$ & 2005 & 08.08 .2014 & Steppe & & Ir.-Tur. \\
\hline Rosa pulverulenta M.Bieb. & 5794 & B7 Tunceli: Pülümür, between Eğimli and & 2341 & 06.08 .2014 & Steppe & & Unk. \\
\hline
\end{tabular}




\begin{tabular}{|c|c|c|c|c|c|c|c|}
\hline & & Kocatepe & & & & & \\
\hline Rosa vanheuckeriana Crép. & 5881 & $\begin{array}{l}\text { B7 Tunceli: Nazımiye, } 6 \mathrm{~km} \mathrm{NE} \text { of } \\
\text { Büyükyurt (Hakis) v. }\end{array}$ & 2050 & 07.08 .2014 & Steppe & & Ir.-Tur. \\
\hline Rubus caesius $\mathrm{L}$. & Obs. & $\begin{array}{l}\text { B7 Tunceli: Çemişgezek, between Gülbahçe } \\
\text { and Dedebeyli }\end{array}$ & 1277 & 17.08 .2014 & Steppe & & Unk. \\
\hline Rubus canescens DC. & 5700 & B7 Tunceli: Center, S of Çimenli village & 987 & 25.07 .2014 & $\begin{array}{l}\text { Field side, } \\
\text { steppe }\end{array}$ & & \begin{tabular}{|l|} 
Euro.- \\
Sib. \\
\end{tabular} \\
\hline Rubus sanctus Shreb. & 6235 & $\begin{array}{l}\text { B7 Tunceli: Center, } 14 \mathrm{~km} \text { from Tunceli to } \\
\text { Pülümür (Pülümür Valley) }\end{array}$ & 965 & 05.09 .2014 & Riverside & & Unk. \\
\hline $\begin{array}{l}\text { Sanguisorba minor L. subsp. } \\
\text { lasiocarpa (Boiss. \& Hausskn.) } \\
\text { Nordborg }\end{array}$ & 4108 & $\begin{array}{l}\text { B7 Tunceli: Mazgirt, } 7 \text { km from Kızılcık to } \\
\text { Göktepe }\end{array}$ & 935 & 02.06 .2014 & Steppe & & Unk. \\
\hline $\begin{array}{l}\text { Sanguisorba minor L. subsp. } \\
\text { minor }\end{array}$ & 4206 & $\begin{array}{l}\text { B7 Tunceli: Ovacık, } 1.5 \text { km from Ovacık- } \\
\text { Tunceli-Yakatarla crossroads to Yakatarla v. }\end{array}$ & 1320 & 04.06 .2014 & Steppe & & Unk. \\
\hline \multirow{2}{*}{ Sorbus umbellata Fritsch } & 5880 & $\begin{array}{l}\text { B7 Tunceli: Nazımiye, } 6 \mathrm{~km} \mathrm{NE} \text { of } \\
\text { Büyükyurt (Hakis) v. }\end{array}$ & 2050 & 07.08 .2014 & \multirow{2}{*}{$\begin{array}{l}\text { Steppe, } \\
\text { Oak forest }\end{array}$} & & \multirow{2}{*}{ Unk. } \\
\hline & 6147 & $\begin{array}{l}\text { B7 Tunceli: Pülümür, } 2 \text { km from Erzincan- } \\
\text { Pülümür-Süleymanuşağ to Süleymanyuşağ }\end{array}$ & 1683 & 27.08 .2014 & & & \\
\hline \multicolumn{8}{|l|}{ RUBIACEAE Juss. } \\
\hline Asperula affinis Boiss. \& A.Huet & 4834 & $\begin{array}{l}\text { B8 Tunceli: Pülümür, } 500 \mathrm{~m} \mathrm{~N} \text { of Balpayam } \\
\text { (3-4 km S of Kırklar v.) }\end{array}$ & 2400 & 18.06 .2014 & Mt. steppe & Rcd. & Ir.-Tur. \\
\hline Asperula arvensis $\mathrm{L}$. & 3977 & $\begin{array}{l}\text { B7 Tunceli: Center, } 1 \mathrm{~km} \text { from Tunceli- } \\
\text { Ovacık-Dedeağaç crossroads to Dedeağaç v. } \\
\text { (Munzur Valley) }\end{array}$ & 1060 & 25.05 .2014 & Steppe & Rcd. & Unk. \\
\hline $\begin{array}{l}\text { Asperula glomerata (M.Bieb.) } \\
\text { Griseb. subsp. condensata } \\
\text { (Ehrend.) Ehrend. var. } \\
\text { condensata }\end{array}$ & 5780 & $\begin{array}{l}\text { B7 Tunceli: Pülümür, } 4.5 \mathrm{~km} \text { from Akdik to } \\
\text { Kovuklu }\end{array}$ & 1600 & 06.08 .2014 & Steppe & Rcd. & Unk. \\
\hline $\begin{array}{l}\text { Asperula glomerata (M.Bieb.) } \\
\text { Griseb. subsp. condensata } \\
\text { (Ehrend.) Ehrend. var. filiformis } \\
\text { Bornm. }\end{array}$ & 4899 & $\begin{array}{l}\text { B7 Tunceli: Pülümür, } 11 \mathrm{~km} \mathrm{NW} \text { of Ardıçlı } \\
\text { (Gersunut) v. (Munzur Mt.) }\end{array}$ & 2380 & 18.06 .2014 & Steppe & Rcd. & Ir.-Tur. \\
\hline $\begin{array}{l}\text { Asperula glomerata (M.Bieb.) } \\
\text { Griseb. subsp. glomerata }\end{array}$ & 4201 & $\begin{array}{l}\text { B7 Tunceli: Ovacık, } 1.5 \mathrm{~km} \text { from Ovacık- } \\
\text { Tunceli-Yakatarla crossroads to Yakatarla v. }\end{array}$ & 1320 & 04.06 .2014 & Steppe & & Unk. \\
\hline \multirow{2}{*}{ Asperula involucrata Wahlenb. } & 4199 & $\begin{array}{l}\text { B7 Tunceli: Ovacık, } 1.5 \mathrm{~km} \text { from Ovacık- } \\
\text { Tunceli-Yakatarla crossroads to Yakatarla v. }\end{array}$ & 1320 & 04.06 .2014 & \multirow{2}{*}{ Steppe } & & \multirow{2}{*}{ Euxine } \\
\hline & 5042 & $\begin{array}{l}\text { B7 Tunceli: Ovacık, between Yazı̈ren and } \\
\text { Gözeler }\end{array}$ & 1400 & 20.06 .2014 & & & \\
\hline $\begin{array}{l}\text { Asperula stricta } \text { Boiss. subsp. } \\
\text { latibracteata (Boiss.) Ehrend. }\end{array}$ & 5606 & B7 Tunceli: Nazımiye, 6 km E of Ayranlı v. & 1800 & 23.07 .2014 & Steppe & $*$ & Ir.-Tur. \\
\hline Callipeltis cucullaris (L.) Steven & 4411 & $\begin{array}{l}\text { B7 Tunceli: Pülümür, between Dereboyu and } \\
\text { Sağlamtaş }\end{array}$ & 1610 & 05.06 .2014 & Steppe & & Ir.-Tur. \\
\hline $\begin{array}{l}\text { Crucianella exasperata Fisch. \& } \\
\text { C.A.Mey. }\end{array}$ & 4515 & B7 Tunceli: Mazgirt, 1 km W of Kayacı v. & 990 & 16.06 .2014 & Steppe & & Unk. \\
\hline $\begin{array}{l}\text { Crucianella gilanica Trin. subsp. } \\
\text { kotschyi (Ehrend.) Ehrend. }\end{array}$ & 5863 & $\begin{array}{l}\text { B7 Tunceli: Nazımiye, } 5 \mathrm{~km} \text { NE of } \\
\text { Büyükyurt (Hakis) v. }\end{array}$ & 1835 & 07.08 .2014 & Steppe & & Ir.-Tur. \\
\hline $\begin{array}{l}\text { Cruciata taurica (Pall. ex Willd.) } \\
\text { Ehrend. }\end{array}$ & 4406 & $\begin{array}{l}\text { B7 Tunceli: Pülümür, between Dereboyu and } \\
\text { Sağlamtaș }\end{array}$ & 1610 & 05.06 .2014 & Steppe & & Ir.-Tur. \\
\hline $\begin{array}{l}\text { Galium incanum Sm. subsp. } \\
\text { elatius (Boiss.) Ehrend. }\end{array}$ & 4655 & $\begin{array}{l}\text { B7 Tunceli: Hozat, between Karacaköy and } \\
\text { Uzundal, around of Sarisaltuk Türbesi }\end{array}$ & 2270 & 17.06 .2014 & Steppe & & Ir.-Tur. \\
\hline Galium megalanthum Boiss. & 4764 & $\begin{array}{l}\text { B7 Tunceli: Pülümür, } 2 \mathrm{~km} \text { from Turnadere } \\
\text { to Közlüce }\end{array}$ & 1575 & 18.06 .2014 & $\begin{array}{l}\text { Rocky } \\
\text { slopes }\end{array}$ & & Ir.-Tur. \\
\hline \multirow[t]{2}{*}{ Galium mite Boiss. \& Hohen. } & 4617 & $\begin{array}{l}\text { B7 Tunceli: Mazgirt, } 5 \mathrm{~km} \text { from Çukur to } \\
\text { Güleç }\end{array}$ & 1430 & 16.06 .2014 & \multirow[t]{2}{*}{ Steppe } & & \multirow[t]{2}{*}{ Ir.-Tur. } \\
\hline & 4789 & B7 Tunceli: Pülümür, SE of Közlüce v. & 1620 & 18.06 .2014 & & & \\
\hline Galium paschale Forssk. & 5884 & $\begin{array}{l}\text { B7 Tunceli: Nazımiye, } 8 \mathrm{~km} \mathrm{NE} \text { of } \\
\text { Büyükyurt (Hakis) v. }\end{array}$ & 1963 & 07.08 .2014 & Oak forest & Rcd. & \begin{tabular}{|l} 
E.Medit. \\
(Mt.)
\end{tabular} \\
\hline $\begin{array}{l}\text { Galium radulifolium Ehrend. \& } \\
\text { Schönb.-Tem. }\end{array}$ & 4727 & $\begin{array}{l}\text { B7 Tunceli: Hozat, } 15 \mathrm{~km} \text { from Ovacık to } \\
\text { Hozat (NE of Halitpınar v.) }\end{array}$ & 1800 & 17.06 .2014 & Steppe & $*$ & Unk. \\
\hline $\begin{array}{l}\text { Galium runcinatum Ehrend. \& } \\
\text { Schönb.-Tem. }\end{array}$ & 4957 & $\begin{array}{l}\text { B7 Tunceli: Ovacık, N of Eskigedik v., } \\
\text { Munzur Mt. }\end{array}$ & 2160 & 19.06 .2014 & $\begin{array}{l}\text { Rocky } \\
\text { areas }\end{array}$ & $*$ & Ir.-Tur. \\
\hline \multirow{2}{*}{ Galium shinasii Yıldırım } & 5155 & $\begin{array}{l}\text { B7 Tunceli: Çemişgezek, E of Anıl village } \\
\text { (Tahar Valley) }\end{array}$ & 1000 & 29.06 .2014 & \multirow{2}{*}{$\begin{array}{l}\text { Rock } \\
\text { crevices }\end{array}$} & \multirow{2}{*}{ Rcd. } & \multirow{2}{*}{ Ir.-Tur. } \\
\hline & 5238 & $\begin{array}{l}\text { B7 Tunceli: Nazımiye, } 800 \mathrm{~m} \text { from } \\
\text { Aşağırabat bridge to Büyükyurt (Hakis) v. }\end{array}$ & 1070 & 30.06 .2014 & & & \\
\hline $\begin{array}{l}\text { Galium verum } \mathrm{L} \text {. subsp. } \\
\text { glabrescens } \text { Ehrend. }\end{array}$ & 5556 & B8 Tunceli: Pülümür, $5 \mathrm{~km} \mathrm{~S}$ of Derindere & 2030 & 22.07 .2014 & Steppe & Red. & Ir.-Tur. \\
\hline Galium verum L. subsp. verum & 5447 & $\begin{array}{l}\text { B7 Tunceli: Hozat, N of Boydaş v. (Tahar } \\
\text { Valley) }\end{array}$ & 1215 & 21.07 .2014 & $\begin{array}{l}\text { Steppe, } \\
\text { dried river } \\
\text { bad }\end{array}$ & & $\begin{array}{l}\text { Euro.- } \\
\text { Sib. }\end{array}$ \\
\hline \multicolumn{8}{|l|}{ SALICACEAE Mirb. } \\
\hline $\begin{array}{l}\text { Populus nigra L. subsp. caudina } \\
\text { (Ten.) Bugala }\end{array}$ & 6229 & $\begin{array}{l}\text { B7 Tunceli: Center, } 5 \mathrm{~km} \text { from Tunceli to } \\
\text { Pülümür (Pülümür Valley) }\end{array}$ & 930 & 05.09 .2014 & Steppe & & Unk. \\
\hline
\end{tabular}




\begin{tabular}{|c|c|c|c|c|c|c|c|}
\hline Populus tremula $\mathrm{L}$. & 5561 & $\begin{array}{l}\text { B8 Tunceli: Pülümür, between Pülümür and } \\
\text { Yedisu, } 2.5 \mathrm{~km} \text { after Derindere v. }\end{array}$ & 1925 & 22.07 .2014 & Riverside & & Unk. \\
\hline Salix acmophylla Boiss. & 6234 & $\begin{array}{l}\text { B7 Tunceli: Center, } 14 \mathrm{~km} \text { from Tunceli to } \\
\text { Pülümür (Pülümür Valley) }\end{array}$ & 965 & 05.09 .2014 & $\begin{array}{l}\text { Damp } \\
\text { places }\end{array}$ & Rcd. & Ir.-Tur. \\
\hline Salix bornmuelleri Hausskn. & 5517 & $\begin{array}{l}\text { B8 Tunceli: Pülümür, between Bardakçı to } \\
\text { Derindere }\end{array}$ & 1980 & 22.07 .2014 & $\begin{array}{l}\text { Stream } \\
\text { side }\end{array}$ & & Ir.-Tur. \\
\hline $\begin{array}{l}\text { Salix pentandroides } \\
\text { A.K.Skvortsov }\end{array}$ & 6203 & $\begin{array}{l}\text { B7 Tunceli: Hozat, } 13 \mathrm{~km} \text { from Hozat to } \\
\text { Ovacık }\end{array}$ & 1972 & 28.08 .2014 & Wetlands & Rcd. & Euxine \\
\hline \multicolumn{8}{|l|}{ SANTALACEAE R.Br. } \\
\hline \multirow{4}{*}{$\begin{array}{l}\text { Arceuthobium oxycedri (DC.) } \\
\text { M.Bieb. }\end{array}$} & 5206 & $\begin{array}{l}\text { B7 Tunceli, Çemişgezek, between } \\
\text { Çemişgezek and Kemaliye, } 1 \mathrm{~km} \text { E of } \\
\text { Dedebeyli village }\end{array}$ & 1275 & 29.06 .2014 & \multirow{4}{*}{$\begin{array}{l}\text { On } \\
\text { Juniperus } \\
\text { sp. }\end{array}$} & \multirow{4}{*}{ Rcd. } & \multirow{4}{*}{$\begin{array}{l}\text { Euro.- } \\
\text { Sib. }\end{array}$} \\
\hline & 6122 & $\begin{array}{l}\text { B7 Tunceli: Çemişgezek, between Gülbahçe } \\
\text { and Dedebeyli }\end{array}$ & 1277 & 17.08.2014 & & & \\
\hline & 6361 & B7 Tunceli: Pertek, E of Dere v. & 1378 & 22.09 .2014 & & & \\
\hline & 6181 & $\begin{array}{l}\text { B7 Tunceli: Center, } 15 \mathrm{~km} \text { from Tunceli- } \\
\text { Pülümür-Sütlüce crossroads to Çıralı v. (10 } \\
\text { km before Çıralı v.) }\end{array}$ & 1844 & 27.08 .2014 & & & \\
\hline \multirow{2}{*}{$\begin{array}{l}\text { Chrysothesium aureum (Jaub. \& } \\
\text { Spach) Hendrych }\end{array}$} & 6531 & B7 Tunceli: Ovacık, 1 km W of Ağaçpınar v. & 1934 & 01.06 .2015 & \multirow{2}{*}{$\begin{array}{l}\text { Steppe } \\
\text { scree }\end{array}$} & \multirow{2}{*}{$\begin{array}{l}* \\
\text { Rcd. }\end{array}$} & \multirow[b]{2}{*}{ Ir.-Tur. } \\
\hline & 4832 & $\begin{array}{l}\text { B8 Tunceli: Pülümür, } 500 \mathrm{~m} \mathrm{~N} \text { of Balpayam } \\
\text { (3-4 km S of Kirklar v.) }\end{array}$ & 2400 & 18.06.2014 & & & \\
\hline Thesium arvense Horv. & 4035 & $\begin{array}{l}\text { B7 Tunceli: Ovacık, } 41 \mathrm{~km} \text { from Tunceli to } \\
\text { Ovacık (Munzur Valley) }\end{array}$ & 1175 & 26.05 .2014 & $\begin{array}{l}\text { Degraded } \\
\text { oak forest, } \\
\text { steppe }\end{array}$ & & $\begin{array}{l}\text { Euro.- } \\
\text { Sib. }\end{array}$ \\
\hline Viscum album $\mathrm{L}$. & 6359 & B7 Tunceli: Pertek, Dere v. & 1268 & 22.09 .2014 & $\begin{array}{l}\text { On Pyrus } \\
\text { sp. }\end{array}$ & & Unk. \\
\hline \multicolumn{8}{|l|}{ SAPINDACEAE Juss. } \\
\hline $\begin{array}{l}\text { Acer monspessulanum L. subsp. } \\
\text { cinerascens (Boiss.) Yalt. }\end{array}$ & 6397 & $\begin{array}{l}\text { B7 Tunceli: Center, } 12 \mathrm{~km} \text { from Tunceli to } \\
\text { Ovacık (Munzur Valley) }\end{array}$ & 1000 & 11.04 .2015 & Riverside & & Ir.-Tur. \\
\hline $\begin{array}{l}\text { Acer monspessulanum L. subsp. } \\
\text { ibericum (M. Bieb.) Yalt. }\end{array}$ & 4011 & $\begin{array}{l}\text { B7 Tunceli: Center, } 17 \mathrm{~km} \text { from Tunceli to } \\
\text { Ovacık (Munzur Valley) }\end{array}$ & 1015 & 25.05 .2014 & Riverside & & Ir.-Tur. \\
\hline \multirow{4}{*}{ Acer platanoides $\mathrm{L}}$. & 5883 & $\begin{array}{l}\text { B7 Tunceli: Nazımiye, } 10 \mathrm{~km} \mathrm{NE} \text { of } \\
\text { Büyükyurt (Hakis) v. }\end{array}$ & 1921 & 07.08.2014 & \multirow{4}{*}{ Oak forest } & & \multirow{4}{*}{$\begin{array}{l}\text { Euro.- } \\
\text { Sib. }\end{array}$} \\
\hline & 5869 & $\begin{array}{l}\text { B7 Tunceli: Nazımiye, } 5 \mathrm{~km} \mathrm{NE} \text { of } \\
\text { Büyükyurt (Hakis) v. }\end{array}$ & 1835 & 07.08 .2014 & & & \\
\hline & 5559 & $\begin{array}{l}\text { B8 Tunceli: Pülümür, between Pülümür and } \\
\text { Yedisu, } 2.5 \mathrm{~km} \text { after Derindere v. }\end{array}$ & 1925 & 22.07 .2014 & & & \\
\hline & 6149 & $\begin{array}{l}\text { B7 Tunceli: Pülümür, } 2 \text { km from Erzincan- } \\
\text { Pülümür-Süleymanuşağ1 to Süleymanyuşağ1 }\end{array}$ & 1683 & 27.08 .2014 & & & \\
\hline \multirow[t]{2}{*}{ Acer tataricum $\mathrm{L}$. } & 5758 & $\begin{array}{l}\text { B7 Tunceli: Pülümür, } 45 \mathrm{~km} \text { from Tunceli to } \\
\text { Pülümür (Pülümür valley) }\end{array}$ & 1200 & 06.08 .2014 & \multirow[t]{2}{*}{ Oak forest } & & \multirow[t]{2}{*}{ Sarmat. } \\
\hline & 5568 & B8 Tunceli: Pülümür, 2 km N of Elmalı & 1400 & 22.07 .2014 & & & \\
\hline \multicolumn{8}{|l|}{ SAXIFRAGACEAE Juss. } \\
\hline Saxifraga cymbalaria L. & 5994 & $\begin{array}{l}\text { B7 Tunceli: Pülümür, } 1.5 \mathrm{~km} \text { from Turnadere } \\
\text { to Közlüce }\end{array}$ & 1500 & 14.08 .2014 & Wetlands & Rcd. & Unk. \\
\hline Saxifraga kotschyi Boiss. & 4283 & $\begin{array}{l}\text { B7 Tunceli: Ovacık, } 3 \text { km N of Işıkvuran v. } \\
\text { Munzur Mt. }\end{array}$ & 2150 & 04.06 .2014 & $\begin{array}{l}\text { Rock } \\
\text { crevices }\end{array}$ & Rcd. & Unk. \\
\hline Saxifraga sibirica $\mathrm{L}$. & 5827 & $\begin{array}{l}\text { B7 Tunceli: Ovacik, } 800 \mathrm{~m} \text { NE of Buyer } \\
\text { Baba Lake }\end{array}$ & 2821 & 06.08 .2014 & $\begin{array}{l}\text { Rocky } \\
\text { areas }\end{array}$ & Rcd. & Unk. \\
\hline \multirow{2}{*}{ Saxifraga tridactylites $\mathrm{L}}$. & 6439 & $\begin{array}{l}\text { B7 Tunceli: Nazımiye, } 33 \mathrm{~km} \text { from Tunceli } \\
\text { to Pülümür (Pülümür Valley) }\end{array}$ & 1061 & 11.04 .2015 & \multirow{2}{*}{$\begin{array}{l}\text { Stream } \\
\text { side, damp } \\
\text { places }\end{array}$} & \multirow{2}{*}{ Red. } & \multirow{2}{*}{$\begin{array}{l}\text { Medit. } \\
\text { (Mt.) }\end{array}$} \\
\hline & 6404 & $\begin{array}{l}\text { B7 Tunceli: Center, } 15 \mathrm{~km} \text { from Tunceli to } \\
\text { Ovacık (Munzur Valley) }\end{array}$ & 994 & 11.04 .2015 & & & \\
\hline \multicolumn{8}{|l|}{ SCROPHULARIACEAE JusS. } \\
\hline $\begin{array}{l}\text { Scrophularia catariifolia } \text { Boiss. } \\
\text { \& Heldr. }\end{array}$ & 6659 & $\begin{array}{l}\text { B7 Tunceli: Pülümür, } 11 \text { km NW of Ardıçlı } \\
\text { (Gersunut) v. (Munzur Mt.) }\end{array}$ & 2516 & 27.06 .2015 & $\begin{array}{l}\text { Rocky } \\
\text { areas }\end{array}$ & Rcd. & Ir.-Tur. \\
\hline Scrophularia crenophila Boiss. & 4708 & $\begin{array}{l}\text { B7 Tunceli: Hozat, between Hozat and } \\
\text { Ovacık, } 2 \mathrm{~km} \text { from Yüceldi to Aşlıca }\end{array}$ & 1970 & 17.06 .2014 & Steppe & Rcd. & Ir.-Tur. \\
\hline $\begin{array}{l}\text { Scrophularia libanotica } \text { Boiss. } \\
\text { subsp. libanotica } \text { var. pontica } \\
\text { R.R.Mill }\end{array}$ & 5979 & $\begin{array}{l}\text { B7 Tunceli: Hozat, } 6 \mathrm{~km} \text { from Hozat to } \\
\text { Dervişcemal }\end{array}$ & 1714 & 13.08 .2014 & Steppe & Rcd. & Ir.-Tur. \\
\hline $\begin{array}{l}\text { Scrophularia libanotica } \text { Boiss. } \\
\text { subsp. libanotica } \text { var. urartuensis } \\
\text { R.R.Mill }\end{array}$ & 4679 & $\begin{array}{l}\text { B7 Tunceli: Hozat, between Karacaköy and } \\
\text { Uzundal, around of Sarısaltuk Türbesi }\end{array}$ & 2270 & 17.06 .2014 & $\begin{array}{l}\text { Rock } \\
\text { crevices }\end{array}$ & $*$ & Ir.-Tur. \\
\hline $\begin{array}{l}\text { Scrophularia luridiflora Fisch. \& } \\
\text { C.A.Mey. }\end{array}$ & 6202 & $\begin{array}{l}\text { B7 Tunceli: Hozat, } 13 \mathrm{~km} \text { from Hozat to } \\
\text { Ovacık }\end{array}$ & 1972 & 28.08 .2014 & Damp soil & $*$ & Ir.-Tur. \\
\hline Scrophularia rimarum Bornm. & 6109 & $\begin{array}{l}\text { B7 Tunceli: Pülümür, } 7 \text { km NW of Ardıçlı } \\
\text { (Gersunut) v. }\end{array}$ & 2002 & 16.08 .2014 & Steppe & & Unk. \\
\hline Scrophularia umbrosa Dumont & 5144 & $\begin{array}{l}\text { B7 Tunceli: Çemişgezek, between Bozağaç } \\
\text { and Ulukale villeges, } 1 \mathrm{~km} \text { E of Ulukale }\end{array}$ & 1080 & 28.06 .2014 & $\begin{array}{l}\text { Dried river } \\
\text { bad }\end{array}$ & & Cosmp. \\
\hline \multirow{2}{*}{$\begin{array}{l}\text { Scrophularia xanthoglossa Boiss. } \\
\text { var. decipiens (Boiss. \& Kotschy) } \\
\text { Boiss. }\end{array}$} & 4005 & $\begin{array}{l}\text { B7 Tunceli: Center, between Tunceli and } \\
\text { Ovacık (Munzur Valley), road of Dilek v. }\end{array}$ & 995 & 25.05 .2014 & \multirow{2}{*}{ Steppe } & \multirow{2}{*}{ Red. } & \multirow{2}{*}{ Ir.-Tur. } \\
\hline & 3715 & B7 Tunceli: Center, between Tunceli and & 1020 & 22.05 .2014 & & & \\
\hline
\end{tabular}




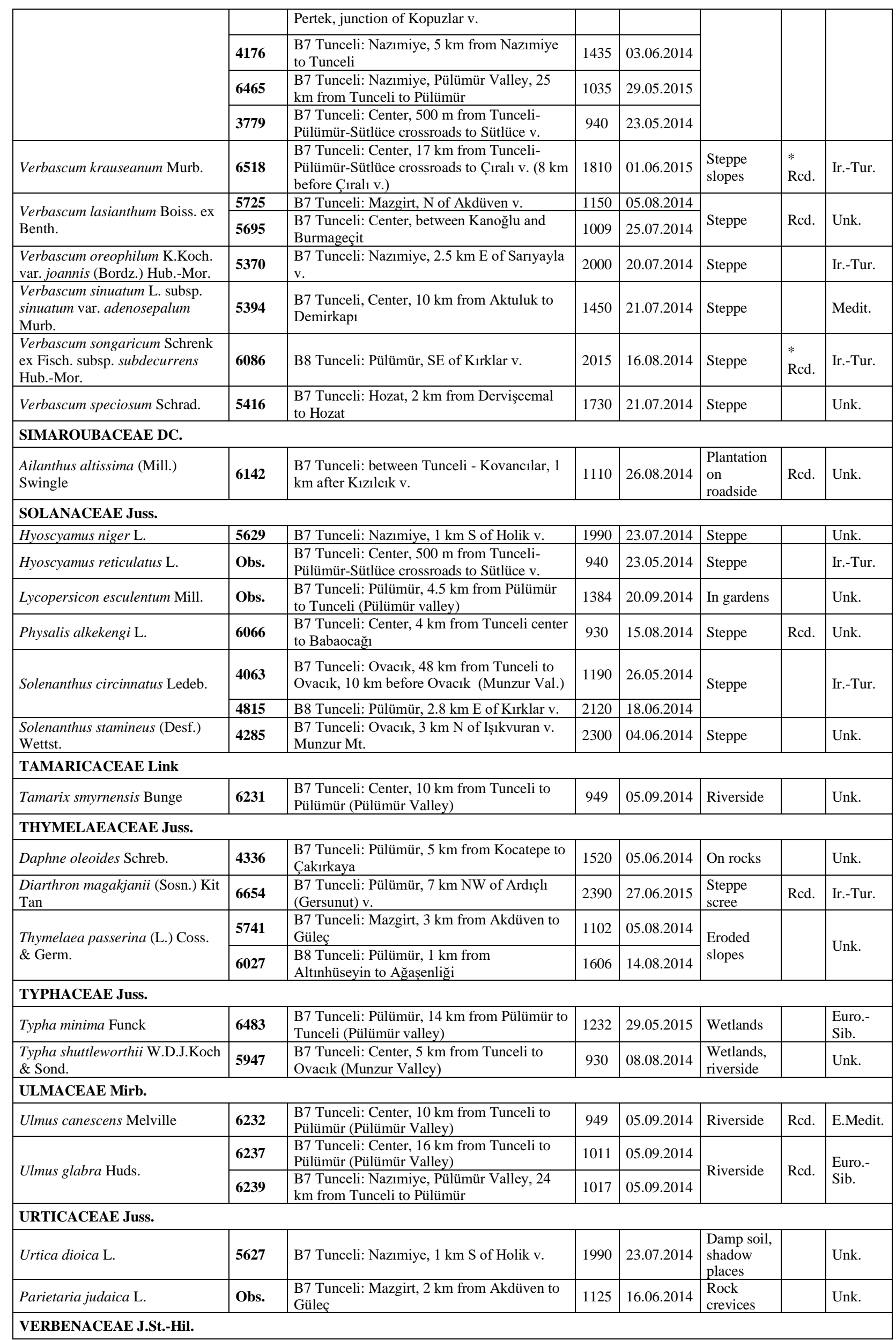




\begin{tabular}{|l|l|l|l|l|l|l|l|}
\hline \multirow{2}{*}{ Verbena officinalis L. } & $\mathbf{4 5 8 3}$ & B7 Tunceli: Mazgirt, 1 km E of Geçitveren v. & 1270 & 16.06 .2014 & \\
\cline { 2 - 7 } & $\mathbf{5 6 3 0}$ & $\begin{array}{l}\text { B7 Tunceli: Nazımiye, 1 km from Dereova to } \\
\text { Ramazanköy }\end{array}$ & 1470 & 23.07 .2014 & Damp soil \\
\cline { 2 - 7 } & $\mathbf{5 6 5 9}$ & $\begin{array}{l}\text { B7 Tunceli: Ovacık, 10 km from Eğripınar to } \\
\text { Yenikonak }\end{array}$ & 1660 & 24.07 .2014 & Unk. \\
\hline ZYGOPHYLLACEAE R.Br. & $\mathbf{O b s .}$ & $\begin{array}{l}\text { B7 Tunceli: Çemişgezek, around of } \\
\text { Yemişdere v. }\end{array}$ & 1000 & 21.06 .2014 & Steppe & Unk. \\
\hline Tribulus terrestris L. & $\mathbf{6 1 2 4}$ & B7 Tunceli: Çemişgezek, Hamamatik & 963 & 17.08 .2014 & $\begin{array}{l}\text { Old } \\
\text { settlement }\end{array}$ & Rcd. & Ir.-Tur. \\
\hline Zygophyllum fabago L. &
\end{tabular}

\section{Discussions}

The identification of these samples resulted 74 families, 386 genera and 922 taxa. The analysis of the phytogeographic regions shows that Irano-Turanian elements 397 (43.1\%), Mediterranean elements 20 (2.2 $\%)$, Europe-Siberian elements 40 (4.3\%), East Mediterranean elements 14 (1.5\%), Euxine element 13 (1.4\%), Hyrcano-Euxine elements $3(0.3 \%)$, Sarmatian 1 $(0.1 \%)$, the cosmopolite or unknown distribution 434 $(47.1 \%)$ (Figure 1). The number of endemic taxa is 150 $(16.3 \%)$.

The families with the most taxa in the research area were Asteraceae (149), Lamiaceae (98), Brassicaceae (82), Caryophyllaceae (82), Fabaceae (56), Apiaceae (49), Rosaceae (35), Poaceae (33), Ranunculaceae (23), and Rubiaceae (20) (Figure 2).

As a result of this study, 294 taxa, 46 of which are endemic, were reported as new recored for Flora of Tunceli province, after the literature were checked (Davis, 1965-1985; Davis et al., 1988; Yıldırımlı, 1995; Güner et al. 2000; Tuzlac1 and Doğan, 2010; Doğan and Tuzlac1, 2010; 2015; Yüce Babacan et al. 2017). According to Ekim et al. (2000), Silene surculosa Hub.-Mor. and Teucrium leucophyllum Montbret \& Aucher ex Benth. species in the EX category were collected from Tunceli for the first time in this study. Until today, 3 new species have been introduced to the scientific world from Tunceli (Gypsophila munzurensis Armağan, Pedicularis munzurdaghensis Armağan, and Gundelia vitekii Armağan). Consider to the potential of studied area (Tunceli province), we anticipate the total number of taxa will reach to around 2000 with the results of other studies and identification of unidentified samples.

In order to become a brand city in the world, many cities take the path of branding by featuring their cultural values and natural sources. On the other hand, studies on conservation of plant gene resources and sustainable use are increasing rapidly. The way of protection is to first identify what exists and to recognize it (Eker et al. 2019). In addition to contributing to the identification and conservation of our biological richness with this study, the data obtained will be a resource for the relevant institutions and organizations working on this subject.

\section{Acknowledgments}

This study is based on field studies as part of "Biodiversity Inventory and Monitoring Works on
Terrestrial and Inland Water Ecosystems of Tunceli Province" carried out by the Tunceli Office of Turkish Republic of Forestry and Water Affairs, General Directorate of Nature Conservation and National Parks. I would like to thank the aforementioned institution, A.H. Gürsönmez and M. Özel. And also, thank Dr. T. Dirmenci, Dr. F. Celep, and Dr. S.M. Pınar for helps.

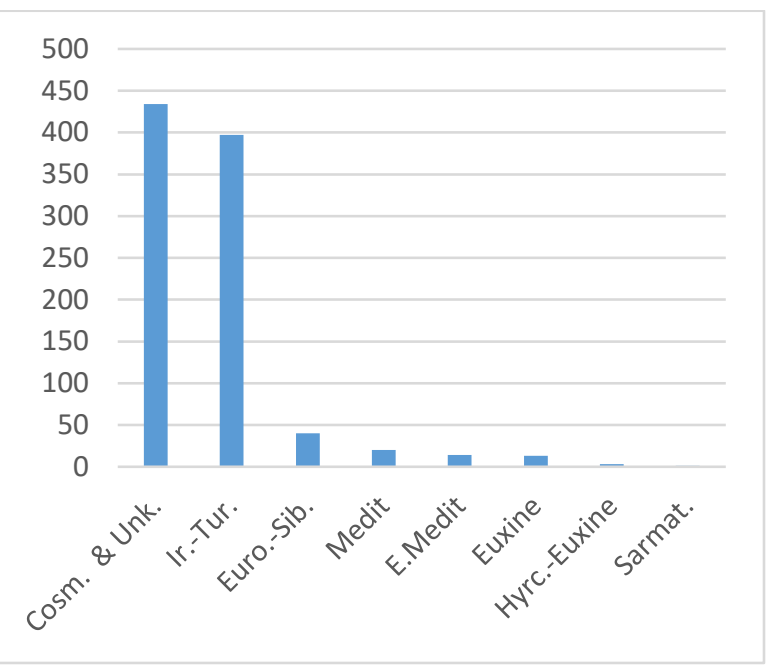

Figure 1. The distribution of taxa depending on phytogeographical regions in this study.

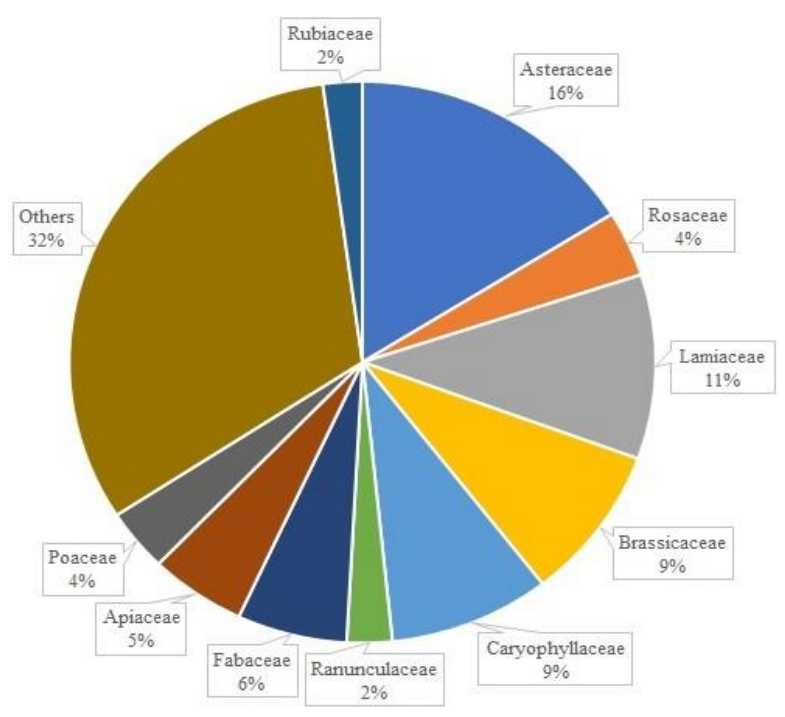

Figure 2. The distribution of families according to number of taxa.

\section{References}

Armağan M (2018). Petaloid monocotyledonous flora of Tunceli Province (Turkey). Hacettepe Journal of Biology and Chemistry 46(2): 199-219.

Bizimbitkiler (2013). http://www.bizimbitkiler.org.tr [accessed 27 February 2020]. 
Davis PH (Ed.) (1965-1985). Flora of Turkey and the East Aegean Islands, Vol. 1-9. Edinburgh: Edinburgh University Press.

Davis PH, Mill RR, Tan K (Ed.) (1988). Flora of Turkey and the East Aegean Islands, Vol. 10. Edinburgh: Edinburgh University Press.

Davis PH (1971). Distribution patterns in Anatolia with particular reference to endemism. In: Davis PH, Harper PC, Hedge IC (Eds.). Plant Life of South-West Asia. The Botanical Society of Edinburgh, pp. 15-27.

Doğan A, Tuzlacı E (2010). Wild edible plants of Pertek (Tunceli-Turkey). Marmara Pharmaceutical Journal 19: 126-135.

Doğan A, Tuzlacı E (2015). Tunceli’nin Bazı Yöresel Bitki Adları. Avrasya Terim Dergisi 3(2): 23-33.

Eker İ, Çelik A, Kaya A, Aydın A (2019). Bolu'nun endemik ve nadir bitkileri. Ankara: Dumat Ofset Matbaacılık San. ve Tic. Ltd. Sti.

Ekim T, Koyuncu M, Duman H, Aytaç Z, Adıgüzel N (2000). Türkiye bitkileri kırmızı kitabı. Ankara: Türkiye Tabiatını Koruma Derneği and Van Yüzüncü Yıl Universitesi

Güner A, Özhatay N, Ekim T, Başer KHC, (Ed.) (2000). Flora of Turkey and the East Aegean Islands, Vol. 11. Edinburgh: Edinburgh University Press.

Gür H (2016). The Anatolian diagonal revisited: Testing the ecological basis of a biogeographic boundary. Zoology in the Middle East 62: 189-199.

IPNI (2020). International plant names index. Published on the Internet; https://www.ipni.org. [accessed 27 February 2020].

MGM (2010). TC Tarım ve Orman Bakanlığı Meteoroloji Genel Müdürlüğü Resmi İstatistikler. https://www.mgm.gov.tr/veridegerlendirme/il-ve-ilceler-istatistik.aspx?m=TUNCELI [accessed 16 March 2020].

Öztürk M, Tatlı A, Özçelik H, Behçet L (2015). General characteristics of flora and vegetation formations of Eastern Anatolia Region and its environs (Türkiye). SDU Journal of Science 10 (1): 23-48.

POWO (2019). Plants of the World Online. Facilitated by the Royal Botanic Gardens, Kew. http://www.plantsoftheworldonline.org/ [accessed 27 February 2020].

Stevens, P. F. (2001 onwards). Angiosperm Phylogeny Website. Version $14, \quad$ July 2017 http://www.mobot.org/MOBOT/research/APweb/. [accessed 15 March 2020]

Thiers B (2016). Index herbariorum: A global directory of public herbaria and associated staff. New York Botanical Garden, Bronx, NY. Available from: http://sweetgum.nybg.org/ih/ [accessed 27 Feb 2020].

Tuzlacı E, Doğan A (2010). Turkish folk medicinal plants, IX: Ovacık (Tunceli). Marmara Pharmaceutical Journal 14: 136-143.

Yıldırımlı Ş (1995). Flora of Munzur Dağları (Erzincan-Tunceli). Ot Sistematik Botanik Dergisi 2(1): 1-78.

Yüce Babacan E, Vitek E, Çakılcıŏglu U (2017). Contributions to the flora of Tunceli (Turkey). International Journal of Nature and Life Sciences (IJNLS) 1(2): 39-66.

WFO (2020). World Flora Online. Published on the Internet; http://www.worldfloraonline.org. [accessed 27 February 2020]. 